\title{
Adsorptive Entfernung von Schwefelverbindungen aus Erdgas
}

Von der Fakultät für Ingenieurwissenschaften, Abteilung Maschinenbau und Verfahrenstechnik der Universität Duisburg-Essen zur Erlangung des akademischen Grades

eines

Doktors der Ingenieurwissenschaften

Dr.-Ing.

genehmigte Dissertation

von

Bastian Steuten

aus

Meerbusch

Gutachter: Univ. Prof. Dr. Dieter Bathen

Univ. Prof. Dr. David W. Agar

Tag der mündlichen Prüfung: 07.03.2014 


\section{Danksagung}

Die vorliegende Arbeit entstand im Rahmen meiner Tätigkeit als wissenschaftlicher Mitarbeiter am Lehrstuhl für Thermische Verfahrenstechnik der Universität Duisburg-Essen.

Ein besonderer Dank gilt dem Lehrstuhlinhaber, Prof. Dr.-Ing. Dieter Bathen, für die Möglichkeit, diese interessante Thematik bearbeiten zu dürfen und die stetige Unterstützung und Förderung während meiner Zeit am Lehrstuhl. Herrn Prof. Agar danke ich für die Übernahme des Korreferats.

Den akademischen Oberräten des Lehrstuhls, Herrn Dr. Christoph Pasel und Herrn Dr. Michael Luckas, danke ich für den jederzeit möglichen fachlichen Austausch und den freundlichen, persönlichen Umgang. Insbesondere Herr Pasel trug wesentlich zum Gelingen des vorliegenden Promotionsvorhabens bei.

Für das tolle Arbeitsklima am Lehrstuhl, die netten Kaffeerunden und die gemeinsamen Freizeitaktivitäten bedanke ich mich bei meinen Kollegen und Kolleginnen, Herrn Pahl, Herrn Schraven, Herrn Gräf, Herrn Ortmann, Herrn Burrichter, Herrn Cox, Frau Hayn, Herrn Chowanietz, Herrn Helmich, Herrn Treese, Frau Vogt, Herrn Urbanczyk und Frau Elsner.

Besonderer Dank gilt Herrn Schraven, der durch seine Erfahrung und seine guten Ideen unverzichtbar beim Aufbau von Versuchsanlagen war und für einen reibungsfreien Ablauf des Laboralltags sorgte. Frau Elsner danke ich die Unterstützung bei den Laborpraktika.

Der experimentelle Umfang dieser Arbeit wurde durch die herausragende Unterstützung zahlreicher Studenten ermöglicht. Besonders zu erwähnen sind unter diesen Tobias Hermann, Roman Schroth, Rustam Karagubenov, Christian Hennisge und Tim Bark.

Einen großen Beitrag leisteten die Fachleute aus der industriellen Praxis. Frau Dr. Angela Siegel, Herrn Dr. Tobias Eckhardt, Herrn Klaus Neumann, Herrn Jörg Nagy und Herrn Peter Engelmann von der BASF Catalysts Germany $\mathrm{GmbH}$ danke ich nicht nur für die Unterstützung des Projektes sondern auch für den regen fachlichen Austausch, die vielen Anregungen und die jederzeit nette und freundliche Atmosphäre bei Treffen und Besuchen.

Meinen Eltern danke ich für die stetige Förderung, den Rückhalt und die Motivation während meiner Studien- und Promotionszeit. Meinen beiden Brüdern Daniel und Benedikt bin ich auf Grund Ihrer Vorbildfunktion zu besonderem Dank verpflichtet.

Meiner Partnerin Pia danke ich für die Geduld, die Sie während der Promotion für mich aufgebracht hat. 


\section{Inhaltsverzeichnis}

1 Einleitung

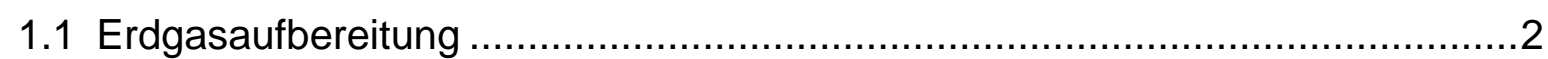

1.2 Stand des Wissens zur adsorptiven Entschwefelung ….................................

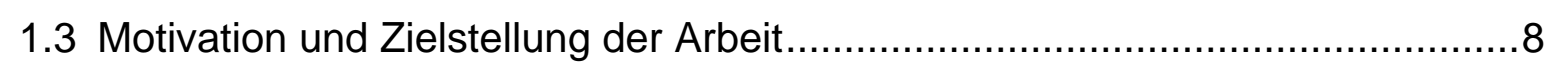

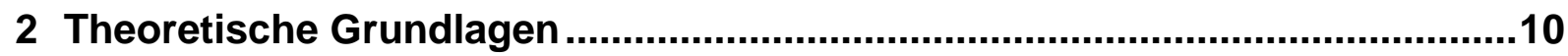

2.1 Begriffsdefinitionen und Grundlagen der Gasphasenadsorption ...................10

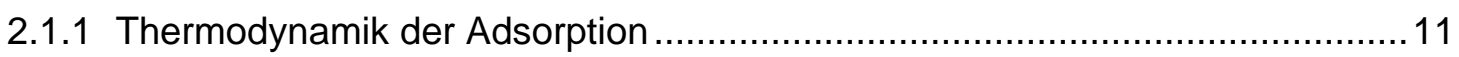

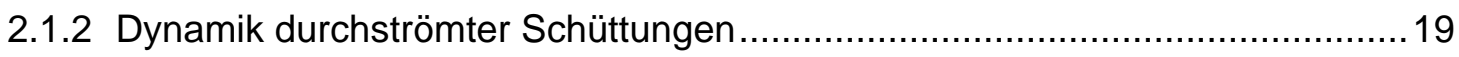

2.1.3 Kinetik der Adsorption und Stofftransportphänomene …………..................21

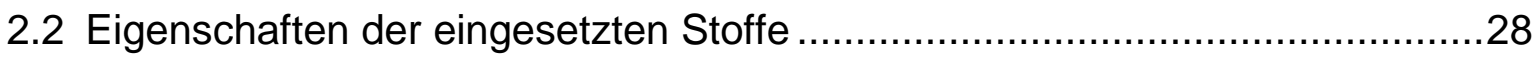

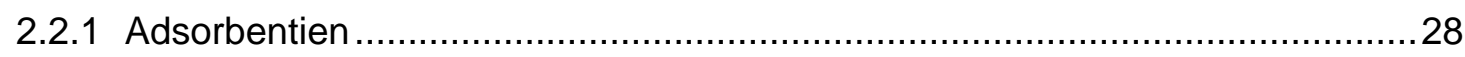

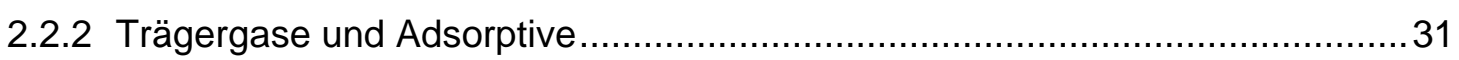

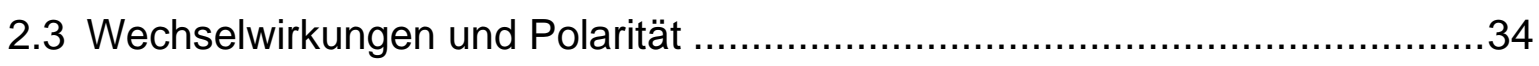

2.4 Quantenmechanische Berechnung von Partialladungen ...............................35

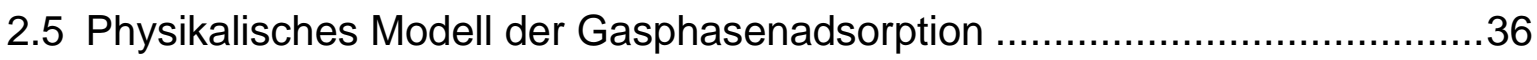

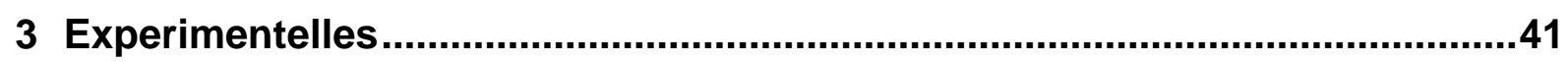

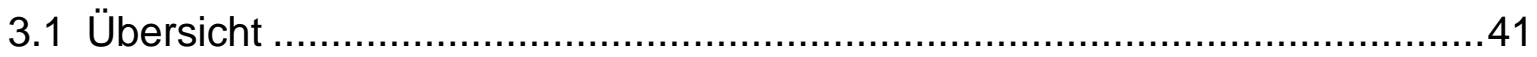

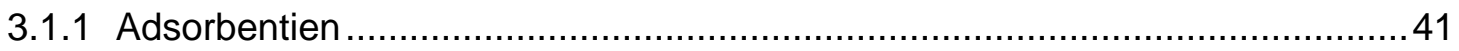

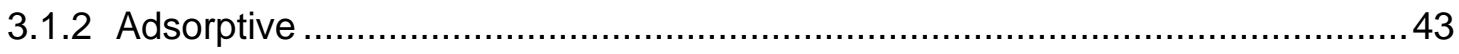

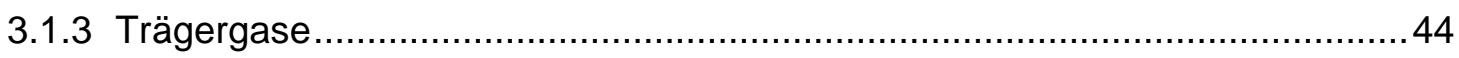

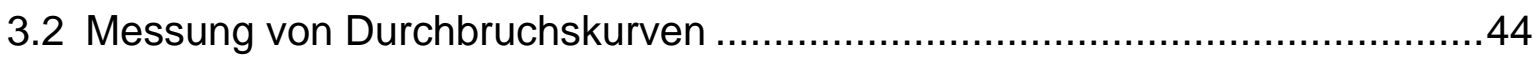

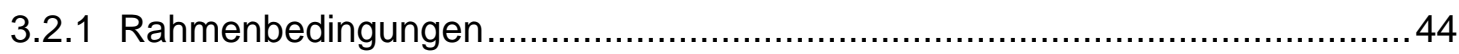

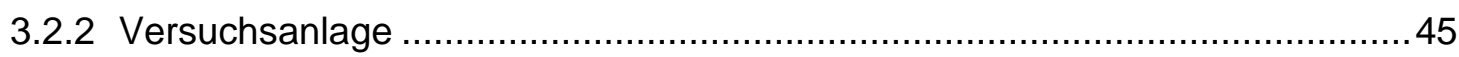

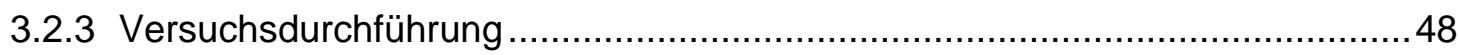

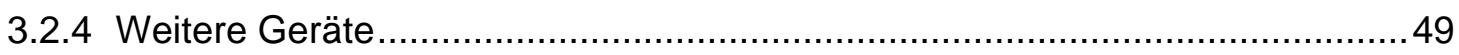

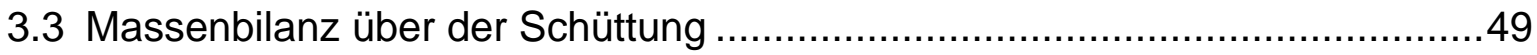

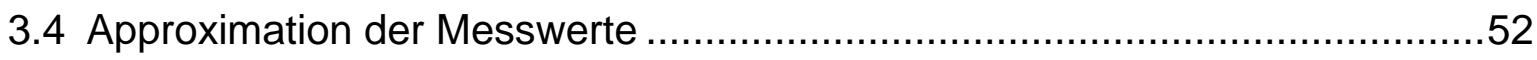

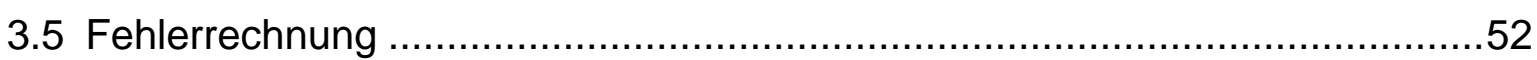

4 Experimentelle Ergebnisse und Diskussion ................................................58

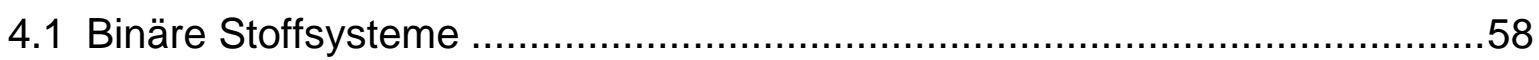

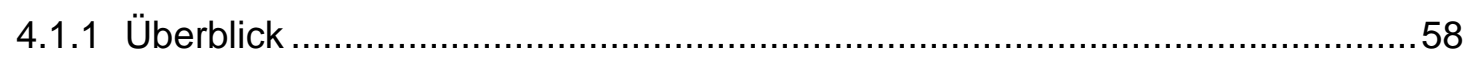

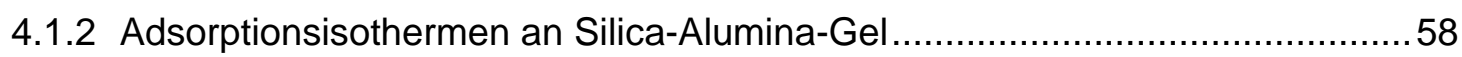

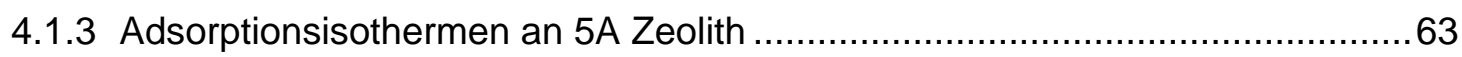

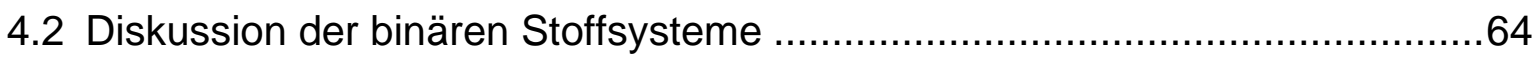

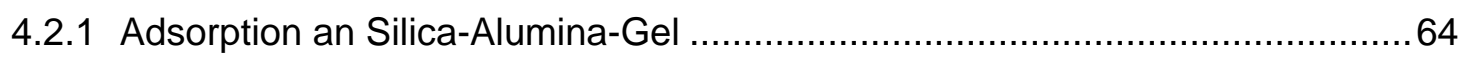

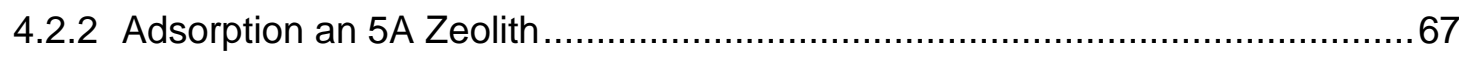




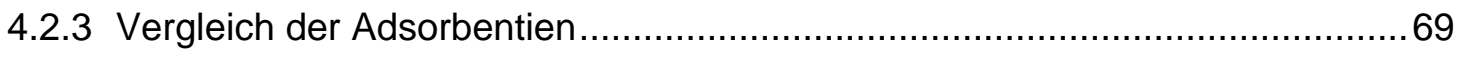

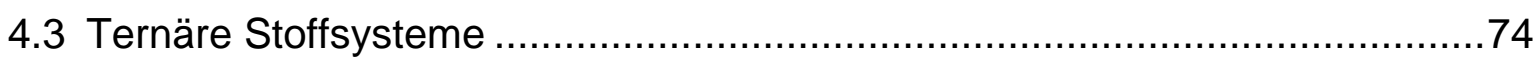

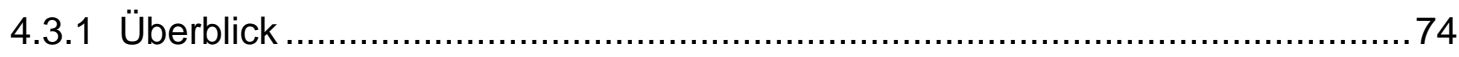

4.3.2 $\mathrm{H}_{2} \mathrm{~S}$-Adsorptionsisothermen an Silica-Alumina-Gel................................... 74

4.3.3 $\mathrm{H}_{2} \mathrm{~S}$-Adsorptionsisothermen an 5A Zeolith ................................................ 79

4.4 Diskussion der ternären Stoffsysteme ............................................... 81

4.4.1 Adsorption an Silica-Alumina-Gel ........................................................... 81

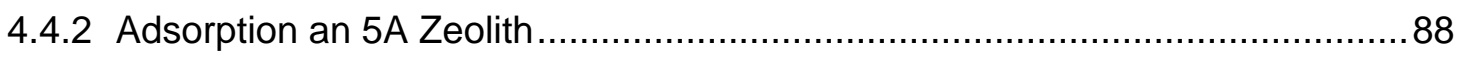

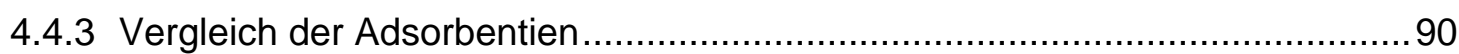

4.4.4 Vorhersage von Mehrkomponentenadsorptionsgleichgewichten....................91

5 Simulation von Adsorptionsprozessen .................................................101

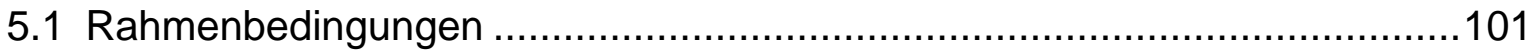

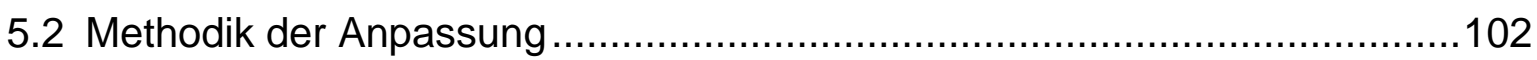

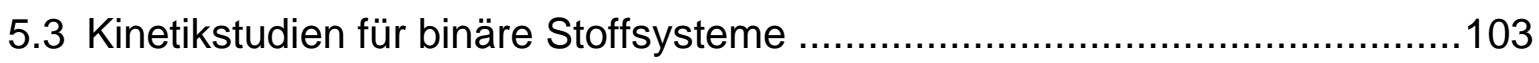

5.3.1 Adsorptionskinetik für Zeolith 5A......................................................... 103

5.3.2 Adsorptionskinetik für Silica-Alumina-Gel ............................................... 107

5.3.3 Vergleich der modellierten Systeme ....................................................... 110

5.4 Kinetische Effekte bei ternären Stoffsystemen ...................................111

6 Zusammenfassung und Ausblick ...........................................................115

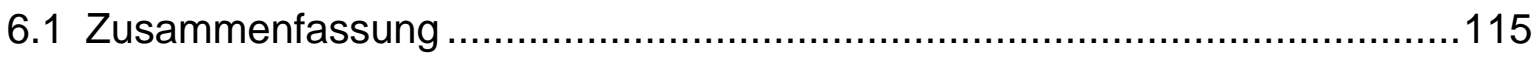

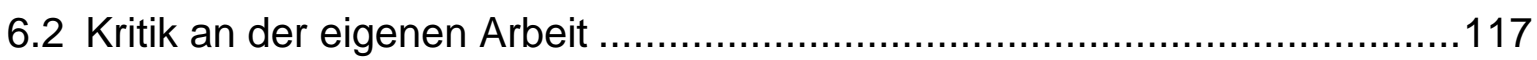

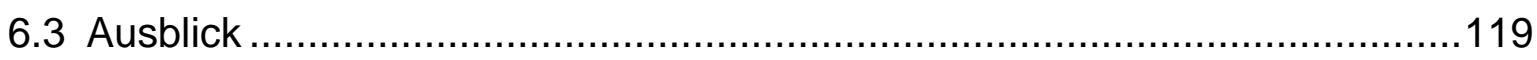

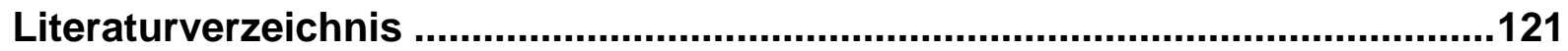

Lebenslauf............................................................................................ 127 


\section{Formelzeichen}

\section{Lateinische Formelzeichen}

\begin{tabular}{|c|c|c|}
\hline Zeichen & Einheit & Bezeichnung \\
\hline$A$ & $m^{2}$ & Fläche \\
\hline$A s p$ & $\mathrm{~m}^{2} / \mathrm{m}^{3}$ & Spezifische Partikeloberfläche \\
\hline$b$ & - & Parameter der Langmuir-Gleichung \\
\hline$c$ & $\mathrm{~kg} / \mathrm{m}^{3}$ & Konzentration \\
\hline$D_{1,2}$ & $\mathrm{~m}^{2} / \mathrm{s}$ & Binärer Diffusionskoeffizient \\
\hline$D K n$ & $\mathrm{~m}^{2} / \mathrm{s}$ & Knudsen-Diffusionskoeffizient \\
\hline DDiff & $\mathrm{m}^{2} / \mathrm{s}$ & Diffusionskoeffizient für Freie Porendiffusion \\
\hline Dges & $\mathrm{m}^{2} / \mathrm{s}$ & Gesamtdiffusionskoeffizient \\
\hline Dax & $\mathrm{m}^{2} / \mathrm{s}$ & Axialer Dispersionskoeffizient \\
\hline$d P$ & $\mathrm{~m}$ & Porendurchmesser \\
\hline G & $\mathrm{kJ}$ & Freie Enthalpie \\
\hline$k_{\text {eff }}$ & $\mathrm{kg} / \mathrm{m}^{2 *} \mathrm{~s}$ & Effektiver Stoffdurchgangskoeffizient \\
\hline$k_{H}$ & $\mathrm{~mol} / \mathrm{mg}$ & Parameter der Henry-Gleichung \\
\hline$k_{F}$ & $\mathrm{~mol} / \mathrm{mg}$ & Parameter der Freundlich-Gleichung \\
\hline$K n$ & - & Knudsen-Zahl \\
\hline$m_{\text {Ads }}$ & $\mathrm{kg}$ & Adsorbensmasse \\
\hline M & $\mathrm{kg} / \mathrm{mol}$ & Molmasse eines Moleküls \\
\hline$\dot{m}$ & $\mathrm{~m} / \mathrm{s}$ & Massenstrom \\
\hline$\dot{n}_{F I}$ & $\mathrm{~mol} / \mathrm{s}$ & Stoffmengenstrom der fluiden Phase \\
\hline$n$ & $\mathrm{~mol} / \mathrm{kg}$ & Beladung \\
\hline$n$ & - & Exponent der Freundlich-Gleichung \\
\hline$p$ & bar & Druck \\
\hline$r_{P}$ & $\mathrm{~m}$ & Partikelradius \\
\hline$R$ & $\mathrm{~J} /\left(\mathrm{mol}{ }^{*} \mathrm{~K}\right)$ & Universelle Gaskonstante $(R=8,3144)$ \\
\hline$R^{2}$ & - & Bestimmtheitsmaß \\
\hline$S$ & $\mathrm{~kJ} / \mathrm{K}$ & Entropie \\
\hline$T$ & Kelvin & Temperatur \\
\hline$t$ & $\mathrm{~s}$ & Zeit \\
\hline$u_{L R}$ & $\mathrm{~m} / \mathrm{s}$ & Leerrohrgeschwindigkeit \\
\hline
\end{tabular}




$\begin{array}{lll}u_{c_{A}} & \mathrm{~m} / \mathrm{s} & \text { Wanderungsgeschwindigkeit der Konzentrationsfront } \\ v & \mathrm{~m} / \mathrm{s} & \text { Geschwindigkeit } \\ \dot{V}_{G} & \mathrm{~m}^{3} / \mathrm{s} & \text { Volumenstrom der fluiden Phase } \\ x & \mathrm{kmol} / \mathrm{kmol} & \text { Stoffmengenanteil in der Adsorbatphase } \\ X & \mathrm{~mol} / \mathrm{kg} & \text { Adsorbensbeladung } \\ X_{G l} & \mathrm{~mol} / \mathrm{kg} & \text { Gleichgewichtsbeladung } \\ X_{m o n} & \mathrm{~mol} / \mathrm{kg} & \text { Parameter der Langmuir-Gleichung } \\ y & \mathrm{~mol} \mathrm{ppm} & \text { Stoffmengenanteil }\end{array}$

\section{Griechische Formelzeichen}

\begin{tabular}{lll} 
Zeichen & Einheit & Bezeichnung \\
\hline$\rho_{P}$ & $\mathrm{~kg} / \mathrm{m}^{3}$ & Partikeldichte \\
$\rho_{S}$ & $\mathrm{~kg} / \mathrm{m}^{3}$ & Scheinbare Dichte einer Adsorbensschüttung \\
$\lambda_{F}$ & $\mathrm{~nm}$ & mittlere freie Weglänge eines Moleküls \\
$\psi_{P}$ & - & Partikelporosität \\
$\psi$ & - & Lückengrad einer Schüttung \\
$\pi$ & $\mathrm{N} / \mathrm{m}$ & Spreizdruck \\
$\pi$ & - & Kreiszahl \\
$V$ & - & Aktivitätskoeffizient in der Adsorbatphase \\
$\Delta_{V}$ & - & Diffusionsvolumen einer Verbindung \\
$\mu_{i}$ & $\mathrm{~J} / \mathrm{mol}$ & Chemisches Potential \\
$\mu_{P}$ & - & Tortuositätsfaktor
\end{tabular}




\section{Abbildungsverzeichnis}

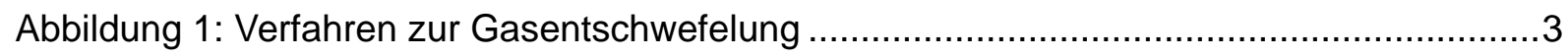

Abbildung 2: Schematische Darstellung der Adsorption nach Keller [31] ........................... 11

Abbildung 3: Henry-Isotherme........................................................................... 12

Abbildung 4: Verschiedene Freundlich-Isothermen................................................... 13

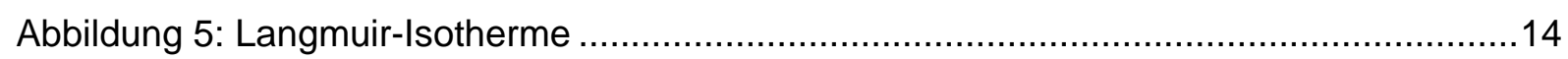

Abbildung 6: Konzentrationsprofil in einer durchströmten Schüttung ................................20

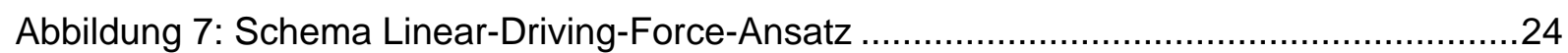

Abbildung 8: Durchbruchskurven bei unterschiedlichen Konzentrationen und konstanter

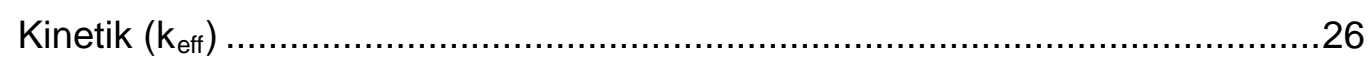

Abbildung 9: Durchbruchskurven bei gleichen Konzentrationen und veränderlicher

Kinetik ( $\left.\mathrm{k}_{\mathrm{eff}}\right)$

Abbildung 10: a, b: Secondary Building Units: Sechsereinfachring und Viererdoppelring,

c: Sodalith-Käfig, d: Elementarzelle des Typ A Zeolith [35]............................30

Abbildung 11: Bilanzraum für die Modellierung der Adsorption .............................................. 36

Abbildung 12: Schema zur Herleitung der Massenbilanz der festen Phase .........................37

Abbildung 13: Schema zur Herleitung der Massenbilanz der fluiden Phase ..........................38

Abbildung 14: Stickstoffisothermen der verwendeten Adsorbentien bei $77 \mathrm{~K}$.

Durchgezogene Linien: Adsorption; gestrichelte Linien: Desorption................43

Abbildung 15: Schematische Darstellung der Versuchsanlage .........................................45

Abbildung 16: Versuchsanlage im Laborbetrieb......................................................46

Abbildung 17: Schematische Darstellung der Bestimmung der Adsorbensbeladung ............50

Abbildung 18: Experimentell bestimmte Durchbruchskurven ( $\mathrm{CO}_{2}$ in $\mathrm{CH}_{4} / 5 \mathrm{~A}$ Zeolith) .......51

Abbildung 19: Schematische Darstellung der Inkrementberechnung der

Durchbruchskurve

Abbildung 20: Isothermen für die Adsorption verschiedener Schwefelverbindungen, $\mathrm{CO}_{2}$

und $\mathrm{H}_{2} \mathrm{O}$ aus $\mathrm{CH}_{4}$ am Silica-Alumina-Gel

Abbildung 21: Isothermen für die Adsorption von $\mathrm{H}_{2} \mathrm{~S}$, COS und $\mathrm{CO}_{2}$ aus $\mathrm{CH}_{4}$ am

Silica-Alumina-gel 60

Abbildung 22: $\mathrm{CO}_{2}$-Adsorptionsisothermen für die verschiedenen Silica-Alumina-Gel-

Varianten

Abbildung 23: $\mathrm{H}_{2} \mathrm{~S}$-Adsorptionsisothermen für die verschiedenen Silica-Alumina-Gel-

Varianten. 
Abbildung 24: Isothermen für die Adsorption verschiedener Schwefelverbindungen, $\mathrm{CO}_{2}$ und $\mathrm{H}_{2} \mathrm{O}$ aus $\mathrm{CH}_{4}$ am Zeolith des Typs $5 \mathrm{~A}$.

Abbildung 25: Bindungsmechanismen an der Silica-Alumina-Gel-Oberfläche. a) $\mathrm{H}_{2} \mathrm{~S}$ -

Bindung über Wasserstoffbrückenbindung; b) $\mathrm{H}_{2} \mathrm{~S}$-Bindung über Lewis-

Säure-Base-Wechselwirkung. .66

Abbildung 26: Isothermen für die Adsorption von Methyl- und Ethylmercaptan aus $\mathrm{CH}_{4}$

am Zeolith 5A

Abbildung 27: Isothermen für die Adsorption von $\mathrm{H}_{2} \mathrm{O}$ aus $\mathrm{CH}_{4}$ und $\mathrm{N}_{2}$ am Zeolith $5 \mathrm{~A}$ und am Silica-Alumina-Gel

Abbildung 28: Isothermen für die Adsorption von $\mathrm{CO}_{2}$ aus Methan am Silica-Alumina-

Gel und 5A Zeolith .72

Abbildung 29: Isothermen für die Adsorption von $\mathrm{H}_{2} \mathrm{~S}$ aus $\mathrm{CH}_{4}$ am Silica-Alumina-Gel bei verschiedenen $\mathrm{CO}_{2}$-Konzentrationen in der Gasmischung ..... .75

Abbildung 30: Isothermen für die Adsorption von $\mathrm{H}_{2} \mathrm{~S}$ aus $\mathrm{CH}_{4}$ am Silica-Alumina-Gel

bei unterschiedlichen Wasserkonzentrationen in der Gasmischung.... .76

Abbildung 31: Isothermen für die Adsorption von $\mathrm{H}_{2} \mathrm{~S}$ aus $\mathrm{CH}_{4}$ an der Silica-Alumina-

Gel-Variante mit 1,5 \% Al-Gehalt bei unterschiedlichen $\mathrm{CO}_{2}-$

Konzentrationen in der Gasmischung .

Abbildung 32: Isothermen für die Adsorption von $\mathrm{H}_{2} \mathrm{~S}$ aus $\mathrm{CH}_{4}$ an der Silica-Alumina-

Gel-Variante mit 0,3\% Al-Gehalt bei unterschiedlichen $\mathrm{CO}_{2}-$

Konzentrationen in der Gasmischung . .78

Abbildung 33: Isothermen für die Adsorption von $\mathrm{H}_{2} \mathrm{~S}$ aus $\mathrm{CH}_{4}$ am Zeolith des Typs $5 \mathrm{~A}$

bei unterschiedlichen $\mathrm{CO}_{2}$-Konzentrationen in der Gasmischung. 79

Abbildung 34: Isothermen für die Adsorption von $\mathrm{H}_{2} \mathrm{~S}$ aus $\mathrm{CH}_{4}$ am Zeolith des Typs $5 \mathrm{~A}$

bei unterschiedlichen $\mathrm{H}_{2} \mathrm{O}-$ Konzentration in der Gasmischung. .80

Abbildung 35: Normierte Durchbruchskurven für die Adsorption von $\mathrm{H}_{2} \mathrm{~S}$ und $\mathrm{CO}_{2}$ am Silica-Alumina-Gel ( $\mathrm{yH}_{2} \mathrm{~S} \approx 2000$ mol-ppm); Rote Symbole: $\mathrm{H}_{2} \mathrm{~S}$-Signal für das binäre Stoffsystem. Grüne und blaue Symbole: Simultan detektierte Signale für $\mathrm{H}_{2} \mathrm{~S}$ (dunkle Farben) und $\mathrm{CO}_{2}$ (helle Farben) für die ternären Stoffsysteme

Abbildung 36: $\mathrm{H}_{2} \mathrm{~S}$-Adsorptionsisothermen für Silica-Alumina-Gel-Varianten bei verschiedenen $\mathrm{CO}_{2}-$ Konzentrationen in der Gasmischung .84 
Abbildung 37: Schematische Verteilung verschiedener Aluminiumplätze der Silica-

Alumina-Gele .86

Abbildung 38: Normierte Durchbruchskurven für ein Experiment des ternären

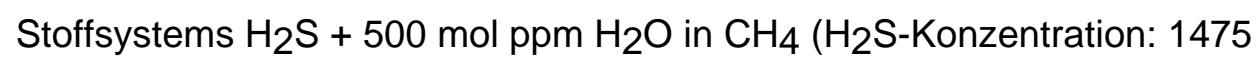

mol ppm) am Silica-Alumina-Gel

Abbildung 39: Normierte Durchbruchskurven für die Adsorption von $\mathrm{H}_{2} \mathrm{~S}$ und $\mathrm{CO}_{2}$ am

Zeolith 5A ( $\mathrm{y}_{\mathrm{H}_{2} \mathrm{~S}} \approx 2000$ mol-ppm); Rote Symbole: $\mathrm{H}_{2} \mathrm{~S}$-Signal für das

binäre Stoffsystem. Grüne und blaue Symbole: Simultan detektierte

Signale für $\mathrm{H}_{2} \mathrm{~S}$ (dunkle Farben) und $\mathrm{CO}_{2}$ (helle Farben) für die ternären

Stoffsysteme

Abbildung 40: Schematische Darstellung simultan detektierter Durchbruchskurven für ein Stoffsystem mit zwei Adsorptiven

Abbildung 41: Beladungen für das ternäre Stoffsystem $\left(\mathrm{H}_{2} \mathrm{~S}+0,5 \mathrm{~mol} \% \mathrm{CO}_{2}\right.$ in $\mathrm{CH}_{4}$ I

5 A Zeolith).

Abbildung 42: Berechnete Adsorptionsgleichgewichte nach IAST (farbige Symbole).

Blaue Symbole: $\mathrm{H}_{2} \mathrm{~S}$-Beladungen, grüne Symbole: $\mathrm{CO}_{2}$-Beladungen in

Abhängigkeit der jeweiligen $\mathrm{H}_{2} \mathrm{~S}$-Konzentration, lila Symbole:

Gesamtbeladung.

Abbildung 43: Vergleich der IAS-Theorie mit der erweiterten Langmuir-Gleichung nach

Markham und Benton (Markham und Benton: farbige Dreiecke; IAS-

Theorie: farbige Rauten)

Abbildung 44: Vergleich der experimentellen Befunde mit den Vorhersagemodellen für

das Stoffsystem $\mathrm{H}_{2} \mathrm{~S}+5 \mathrm{~mol} \% \mathrm{CO}_{2}+95 \mathrm{~mol}_{\%} \mathrm{CH}_{4} / 5 \mathrm{~A}$ Zeolith

Abbildung 45: Isothermen für die Adsorption von $\mathrm{H}_{2} \mathrm{~S}$ aus $\mathrm{CH}_{4}$ und $\mathrm{CO}_{2}$ aus $\mathrm{CH}_{4}$ an $5 \mathrm{~A}$

Zeolith .98

Abbildung 46: Vergleich der experimentellen Befunde mit der Vorhersage nach IAST für

das Stoffsystem $\mathrm{H}_{2} \mathrm{~S}+0,5 \mathrm{~mol} \% \mathrm{CO}_{2}$ in $\mathrm{CH}_{4}$ / Silica-Alumina-Gel..... 100

Abbildung 47: Iterative Anpassung der keff-Werte an eine experimentell bestimmte

Durchbruchskurve am Beispiel $\mathrm{CO}_{2}$ in $\mathrm{CH}_{4} / 5 \mathrm{~A}$ Zeolith

Abbildung 48: Oben: Normierte gemessene und modellierte Durchbruchskurven für die Adsorption von $\mathrm{CO}_{2}$ aus $\mathrm{CH}_{4}$ am Zeolithen des Typs 5A. Symbole:

Messwerte; Schwarze Linien: Modellierung. Unten: Entsprechende $\mathrm{CO}_{2}-$ Isotherme mit Messwerten. Symbole: Messwerte; Gestrichelte Linie:

Langmuir-Anpassung.... 
Abbildung 49: Schematischer Verlauf des effektiven Stoffdurchgangskoeffizienten in Abhängigkeit von der Konzentration. Schwarze Linie: Beobachteter Verlauf für die modellierten Stoffsysteme; Gestrichelte Linie: Erwarteter Verlauf gemäß Gleichung 2.36 .

Abbildung 50: Angepasste keff-Werte für Durchbruchskurven bei verschiedenen

Konzentrationen für das Stoffsystem $\mathrm{CO}_{2}$ in $\mathrm{CH}_{4}$ am $5 \mathrm{~A}$ Zeolith. 106

Abbildung 51: Oben: Normierte gemessene und modellierte Durchbruchskurven für die Adsorption von $\mathrm{H}_{2} \mathrm{~S}$ aus $\mathrm{CH}_{4}$ am Silica-Alumina-Gel. Symbole:

Messwerte; Schwarze Linien: Modellierung. Unten: Entsprechende $\mathrm{H}_{2} \mathrm{~S}$ Isotherme. Symbole: Messwerte; Gestrichelte Linie: Henry-Anpassung 108

Abbildung 52: Vergleich normierter Durchbruchskurven der Adsorption von $\mathrm{CO}_{2}$ für die binären Experimente $\left(0,5 \mathrm{~mol} \% \mathrm{CO}_{2}\right.$ in $\mathrm{CH}_{4}, 5 \mathrm{~mol} \% \mathrm{CO}_{2}$ in $\left.\mathrm{CH}_{4}\right)$ und die ternären Stoffsysteme $\left(\mathrm{H}_{2} \mathrm{~S}+0,5 \mathrm{~mol} \%\right.$ in $\mathrm{CH}_{4}, \mathrm{H}_{2} \mathrm{~S}+5 \mathrm{~mol} \% \mathrm{CO} 2$ in $\left.\mathrm{CH}_{4}\right)$

Abbildung 53: Erneute Darstellung der Abbildung 33 und Abbildung 39 


\section{Tabellenverzeichnis}

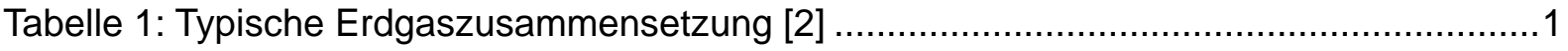

Tabelle 2: Berechnungsmethodik und Parameter für die Partialladungen ...........................35

Tabelle 3: Strukturelle Eigenschaften der verwendeten Adsorbentien ................................42

Tabelle 4: Strukturelle Eigenschaften der modifizierten Silica-Alumina-Gele ........................42

Tabelle 5: Physikalische Eigenschaften der verwendeten Adsorptive ................................44

Tabelle 6: Physikalische Eigenschaften der verwendeten Trägergase [38] .........................44

Tabelle 7: Beispielhaftes Volumenstromverhältnis für einen Versuch ..................................46

Tabelle 8: Übersicht über die Geometrie der Adsorber .................................................. 48

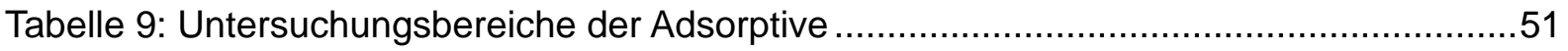

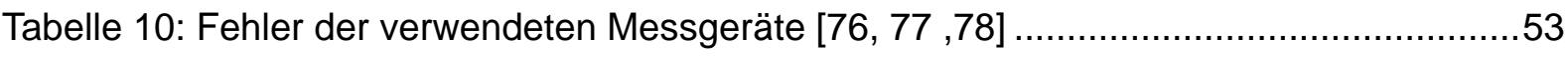

Tabelle 11: Beispielhafte Berechnung des relativen systematischen Gesamtfehlers............53

Tabelle 12: Beispielhafte Wiederholungsmessung für das binäre Stoffsystem $\mathrm{H}_{2} \mathrm{~S}$ in $\mathrm{CH}_{4} \ldots 57$

Tabelle 13: Angepasste Isothermenparameter binärer Stoffsysteme für das Silica-

Alumina-Gel

Tabelle 14: Angepasste Isothermenparameter der binären Stoffsysteme $\left(\mathrm{H}_{2} \mathrm{~S}\right.$ in $\mathrm{CH}_{4}$ und $\mathrm{CO}_{2}$ in $\mathrm{CH}_{4}$ ) für alle Silica-Alumina-Gel-Varianten

Tabelle 15: Parameter der Isothermenanpassungen für binäre Stoffsysteme an 5A

Zeolith..... .64

Tabelle 16: Dipolmomente der Adsorptivmoleküle [68, 69, 70]

Tabelle 17: $\mathrm{H}_{2} \mathrm{~S}$-Adsorptionskapazitäten an Zeolithen im Bereich von 2000 mol ppm (Werte umgerechnet)

Tabelle 18: Berechnete Partialladungen des Schwefelatoms für die untersuchten Mercaptane

Tabelle 19: Parameter der Isothermenanpassungen für die ternären Stoffsysteme $\left(\mathrm{H}_{2} \mathrm{~S}+\right.$ $\mathrm{CO}_{2}$ in $\mathrm{CH}_{4}$ ) an Silica-Alumina-Gel .75

Tabelle 20: Parameter der Isothermenanpassungen für die ternären Stoffsysteme $\left(\mathrm{H}_{2} \mathrm{~S}+\right.$ $\mathrm{CO}_{2}$ in $\mathrm{CH}_{4}$ ) an Silica-Alumina-Gel

Tabelle 21: Parameter der Isothermenanpassungen für die ternären Stoffsysteme $\left(\mathrm{H}_{2} \mathrm{~S}+\right.$ $\mathrm{CO}_{2}$ in $\mathrm{CH}_{4}$ ) an den modifizierten Silica-Alumina-Gel-Varianten

Tabelle 22: Parameter der Isothermenanpassungen für die ternären Stoffsysteme $\left(\mathrm{H}_{2} \mathrm{~S}+\right.$ $\mathrm{CO}_{2}$ in $\mathrm{CH}_{4}$ ) am Zeolith $5 \mathrm{~A}$. 
Tabelle 23: Angepasste Isothermenparameter für die Stoffsysteme $\mathrm{H}_{2} \mathrm{~S}+\mathrm{H}_{2} \mathrm{O}$ in $\mathrm{CH}_{4}$

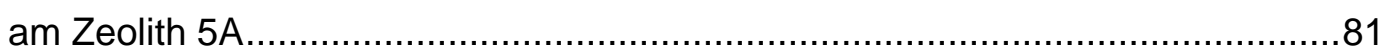

Tabelle 24: Berechnete Diffusionskoeffizienten nach Fuller (vgl. Gleichung 2.34)..............102

Tabelle 25: Effektive Stoffdurchgangskoeffizienten für den 5A Zeolithen ............................105

Tabelle 26: Effektive Stoffdurchgangskoeffizienten der Henry-Systeme am Silica-

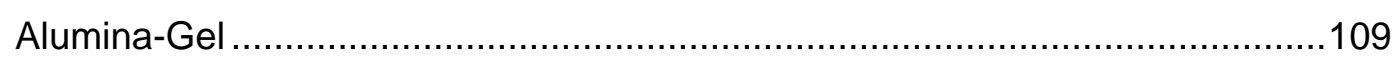

Tabelle 27: Effektive Stoffdurchgangskoeffizienten der Freundlich-Systeme am Silica-

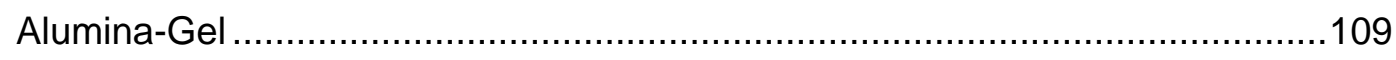

Tabelle 28: Nach Hirschfelder abgeschätzte Knudsen-Zahlen....................................... 110 


\section{$1 \quad$ Einleitung}

Erdgas ist einer der wichtigsten fossilen Energieträger. Neben Erdöl und Kohle besitzt Erdgas den größten Anteil am weltweiten Verbrauch primärer fossiler Energieträger [1]. Auf Grund der großen weltweiten Reserven von ca. 208,4 Billionen $\mathrm{m}^{3}$ [1] und der geringen spezifischen $\mathrm{CO}_{2}$-Emission bei der Verbrennung im Vergleich zu anderen fossilen Energieträgern wird Erdgas auch eine wichtige Rolle in der zukünftigen Energieversorgung spielen. Ähnlich wie Erdöl entsteht Erdgas durch anaerobe Zersetzungsprozesse organischen Materials. Dadurch handelt es sich immer um ein Gasgemisch, dessen Zusammensetzung je nach Ausgangsmaterial, Förderstätte und -tiefe stark variieren kann. Tabelle 1 gibt eine Auflistung der Hauptbestandteile eines typischen Erdgases.

Tabelle 1: Typische Erdgaszusammensetzung [2]

\begin{tabular}{|l|l|l|}
\hline Methan & $\mathrm{CH}_{4}$ & \multirow{2}{*}{$0-90 \%$} \\
\hline Ethan & $\mathrm{C}_{2} \mathrm{H}_{6}$ & \multirow{2}{*}{$0-20 \%$} \\
\cline { 1 - 2 } Propan & $\mathrm{C}_{3} \mathrm{H}_{8}$ & \\
\hline Butan & $\mathrm{C}_{4} \mathrm{H}_{10}$ & $0-8 \%$ \\
\hline Kohlendioxid & $\mathrm{CO}_{2}$ & $0-0.2 \%$ \\
\hline Sauerstoff & $\mathrm{O}_{2}$ & $0-5 \%$ \\
\hline Stickstoff & $\mathrm{N}_{2}$ & Spuren-Sättigung \\
\hline Wasser & $\mathrm{H}_{2} \mathrm{O}$ & $0-5 \%$ \\
\hline Schwefelwasserstoff & $\mathrm{H}_{2} \mathrm{~S}$ & Spuren \\
\hline Edelgase & $\mathrm{A}, \mathrm{He}, \mathrm{Ne}, \mathrm{Xe}$ & \\
\hline
\end{tabular}

Kommerziell nutzbares Erdgas besteht als Hauptkomponente aus Methan $\left(\mathrm{CH}_{4}\right)$. Zusätzlich können Alkane wie Ethan $\left(\mathrm{C}_{2} \mathrm{H}_{6}\right)$, Propan $\left(\mathrm{C}_{3} \mathrm{H}_{8}\right)$ und Butan $\left(\mathrm{C}_{4} \mathrm{H}_{10}\right)$ enthalten sein. Weitere Nebenbestandteile sind Kohlendioxid $\left(\mathrm{CO}_{2}\right)$, Sauerstoff $\left(\mathrm{O}_{2}\right)$, Stickstoff $\left(\mathrm{N}_{2}\right)$, Spuren von Edelgasen (z.B. Ar, He, Ne, Xe), Wasser $\left(\mathrm{H}_{2} \mathrm{O}\right)$ und Schwefelwasserstoff $\left(\mathrm{H}_{2} \mathrm{~S}\right)$. Zu diesen Verbindungen können Verunreinigungen im Spurenbereich hinzukommen. Von besonderem Interesse sind Schwefelverbindungen wie beispielsweise Carbonylsulfid (COS), Kohlenstoffdisulfid $\left(\mathrm{CS}_{2}\right)$, Mercaptane (z.B. $\mathrm{CH}_{3} \mathrm{SH}, \mathrm{C}_{2} \mathrm{H}_{5} \mathrm{SH}$ ) oder flüchtige organische Verbindungen (z.B. Benzol, Toluol, Xylol).

Um Erdgas für verschiedenste technische Applikationen nutzbar zu machen, müssen darin enthaltene Verunreinigungen abgetrennt werden. Die Aufbereitung des Erdgases erfolgt im Anschluss an die Förderung in mehreren Stufen. Üblicherweise werden „saure Verunreinigungen“ wie Schwefelverbindungen oder Kohlendioxid zuerst abgetrennt. Darauffolgend wird 
das Erdgas auf sehr niedrige Wassergehalte gebracht. Zuletzt folgen Verfahrensschritte zur Gewinnung von Kohlenwasserstoffen, Verringerung des Stickstoffgehaltes und zur Gewinnung von Edelgasen wie Helium [3]. Da der thematische Schwerpunkt dieser Arbeit im Bereich der Abtrennung „saurer“ Verbindungen liegt, gibt der folgende Abschnitt einen Überblick über industriell etablierte Trennverfahren zur Entschwefelung und Abtrennung von Kohlendioxid aus Erdgas.

\subsection{Erdgasaufbereitung}

Saure Verbindungen wie Schwefelkomponenten und Kohlendioxid müssen auf Grund Ihrer nachteiligen Eigenschaften abgetrennt werden (vgl. Abschnitt 2.2.2). Dabei ist die Abtrennung dieser Verbindungen aus der Gasphase nicht ausschließlich auf Erdgasanwendungen zu beschränken. Bestrebungen, z.B. regenerativ erzeugte Gase wie Biogas in Erdgasnetze einzuspeisen, erfordern ähnliche Verfahren, um gesetzliche Spezifikationen einzuhalten [4]. Aus diesem Grund besteht bei der Aufbereitung verschiedenster Rohgase eine große Schnittmenge in der Verfahrensauswahl.

Prinzipiell lassen sich die Verfahren anhand ihrer Wirkweisen gruppieren:

- Physikalische Trennverfahren

- Chemische Trennverfahren

- Biologische Trennverfahren

Hiervon werden die physikalisch und chemisch wirkenden Trennverfahren im industriellen Maßstab eingesetzt [3]. Zum vertieften Verständnis der biologischen Verfahren sei auf die relevante Literatur verwiesen [5].

Anhand der eingesetzten Hilfsstoffe lassen sich die physikalischen und chemischen Aufbereitungsverfahren nach den Grundoperationen der thermischen Verfahrenstechnik gliedern. Abbildung 1 gibt einen Überblick über die gängigen Gasentschwefelungsverfahren. 


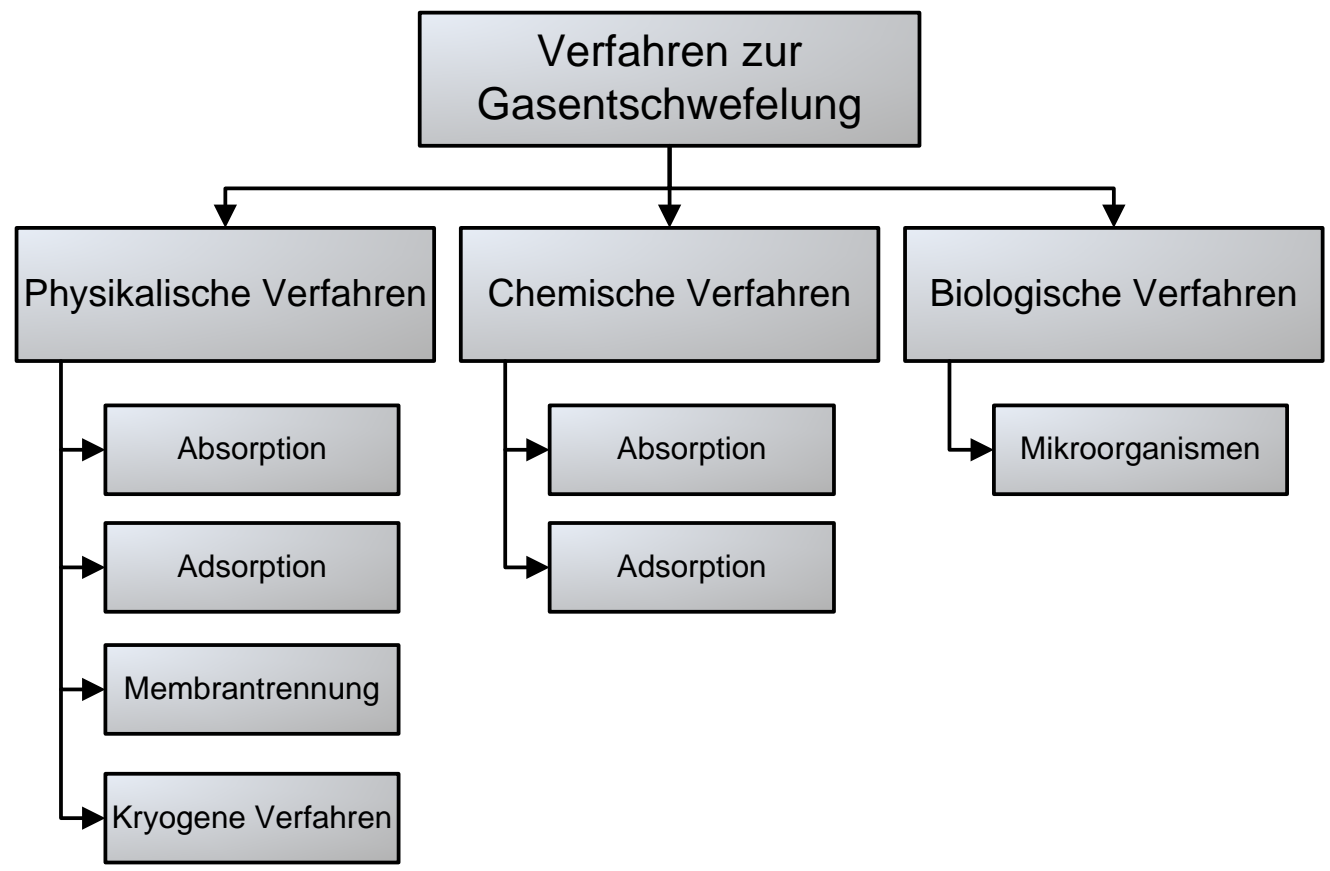

Abbildung 1: Verfahren zur Gasentschwefelung

Im Folgenden wird auf einige der Verfahren kurz eingegangen.

\section{Absorption}

Bei den absorptiven Gasentschwefelungsverfahren haben sich diverse Lösungsmittel etabliert. Eine Unterscheidung der Verfahren lässt sich durch die Wirkweise des Lösungsmittels treffen. Es gibt physikalische, chemische und auch hybride Waschprozesse.

Unter den chemischen Verfahren zählt die Gaswäsche mit Aminen zu den bekanntesten. Dabei werden die „sauren“ Verunreinigen im Erdgas von einer wässrigen Aminlösung absorbiert. Oft verwendete Amine sind Monoethanolamin (MEA), Diethanolamin (DEA), Triethanolamin (TEA), Methyldiethanolamin (MDEA), Diglycolamin (DGA).

Des Weiteren gibt es Verfahren mit gemischten Aminen oder chemisch-physikalisch wirkenden Aminlösungen, denen Additive zugeführt werden, um die Selektivität zu verbessern. Als Beispiele hierfür seien der Sulfinol- und der FLEXSORB-Prozess genannt [6].

Ein weiteres, der Aminwäsche sehr ähnliches Verfahren, ist die Gaswäsche mittels gelöster alkalischer Salze. Die hierfür verwendeten Chemikalien werden auch für die Imprägnierung von Aktivkohlen eingesetzt (vgl. Abschnitt „Adsorption“). Oft verwendete Chemikalien sind: Natriumhydroxid $(\mathrm{NaOH})$, Kaliumhydroxid $(\mathrm{KOH})$, Natriumcarbonat $\left(\mathrm{Na}_{2} \mathrm{CO}_{3}\right)$, Kaliumcarbonat $\left(\mathrm{K}_{2} \mathrm{CO}_{3}\right)$, Kaliumpermanganat $\left(\mathrm{KMnO}_{4}\right)$. 
Eine weitere Möglichkeit der chemischen Gaswäsche stellt die Verwendung wässrig gelöster Chelatkomplexe dar [7]. Als Beispiele dafür seien der SULFEROX- und der LO-CAT-Prozess genannt [8].

Neben den chemisch wirkenden Gaswaschprozessen gibt es auch Prozesse, die rein physikalisch ablaufen. Hieraus seien beispielhaft die Druckwasserwäsche, der Rectisol- und der Selexol-Prozess genannt [6].

Physikalische Absorptionsprozesse basieren auf der Tatsache, dass sich die sauren Verunreinigungen bei Prozessbedingungen viel besser in dem jeweiligen Lösungsmittel lösen als das Matrixgas Methan oder weitere Wertstoffe. Beim Rectisol-Verfahren wird Methanol als Lösungsmittel verwendet. Der Prozess läuft bei Temperaturen von ca. $-40^{\circ} \mathrm{C}$ ab. Der SelexolProzess wird mit Polyethylenglycoldimethylether als Lösungsmittel betrieben. Durch Veränderung von Prozessparametern wie Temperatur und Druck sind diese Lösemittel gut zu regenerieren.

\section{Adsorption}

Wie für den Fall der absorptiven Verfahren lassen sich die adsorptiven Verfahren anhand ihrer Wirkweise unterteilen. Industriell etabliert haben sich sowohl physisorptive als auch chemisorptive Adsorbentien [9].

Die chemisorptiven Materialien sind mit den chemisch wirkenden Wäschen zu vergleichen. Meist bedient man sich ähnlicher Chemikalien, um eine Entschwefelung des Gases durchzuführen. Anstatt diese Chemikalien in eine Lösung zu bringen, werden sie auf poröse Trägermaterialien aufgebracht. Als Trägermaterialien für diese Imprägnierungsverfahren fungieren physisorptive Standardmaterialien wie z. B. Aktivkohlen. Dadurch wird der Imprägnierungschemikalie eine große Oberfläche zur Verfügung gestellt. Je nach Applikation sind folgende imprägnierte Aktivkohlen zur Entschwefelung gebräuchlich [10]:

- Alkalische Imprägnierungen: $\mathrm{NaOH}, \mathrm{Na}_{2} \mathrm{CO}_{3}, \mathrm{KOH}, \mathrm{K}_{2} \mathrm{CO}_{3}, \mathrm{KI}, \mathrm{KMnO}_{4}$

- Metalloxide: z. B. Eisenoxid, Manganoxid

Daneben gibt es spezielle Applikationen, bei denen eine Entschwefelung mit Hilfe von Metalloxiden wie Kupfer- oder Zinkoxiden durchgeführt wird. Alle genannten Materialien weisen zwar gute Abtrennleistungen und große Kapazitäten auf, jedoch lassen sie sich meist nur teilweise oder gar nicht unter wirtschaftlichen Betriebsbedingungen regenerieren. Dies spricht für eine genauere Betrachtung von physisorptiven Adsorbentien wie z. B. Zeolithen oder Silica-Gelen, die unter wirtschaftlichen Bedingungen regenerierbar sind. Da der 
Schwerpunkt dieser Arbeit die Untersuchung solcher Adsorbentien behandelt, wird der Stand des Wissens zu diesem Gebiet in Abschnitt 1.2 vertieft.

Neben den bereits vorgestellten Möglichkeiten zur Abtrennung saurer Verbindungen gibt es noch weitere Verfahren, die nicht weiter vertieft werden sollen, der Vollständigkeit halber jedoch erwähnt seien.

Die Abtrennung saurer Komponenten mit Hilfe von Membranen stellt für bestimmte Applikationen eine Alternative zu den bereits vorgestellten Verfahren dar. Die Selektivität einer Membran ergibt sich aus den unterschiedlichen Permeationsraten der verschiedenen Moleküle. Moderne Membranen zur Gasaufbereitung bestehen üblicherweise aus PolymerMaterialien. Sie werden als Wickel- oder Hohlfasermodule verbaut. Membranen werden in Verbindung mit anderen Trennverfahren eingesetzt, um Aufreinigungen bis in den Spurenbereich zu erreichen [6].

Ein weiteres Verfahren, um z. B. Schwefelwasserstoff zu entfernen, ist die biologische Konversion zu elementarem Schwefel. Industriellen Einsatz findet dieses Verfahren im ShellPaques/Thiopaq-Prozess. Dieser Prozess kombiniert verschiedene Trennverfahren. $\mathrm{H}_{2} \mathrm{~S}$ wird zunächst in alkalischer Lösung absorbiert. Entstandene Sulfide werden in einem zweiten Verfahrensschritt direkt zu elementarem Schwefel oxidiert [11].

Sowohl Membran- als auch biologische Verfahren finden sich nur in Nischenanwendungen. Haupttrennverfahren sind Adsorption und Absorption.

\subsection{Stand des Wissens zur adsorptiven Entschwefelung}

Die adsorptive Entschwefelung wird weltweit von mehreren Forschungsgruppen untersucht, so dass für verschiedene Schwefelverbindungen experimentelle Daten zur Adsorption vorliegen. Unter den Schwefelverbindungen im Erdgas hat Schwefelwasserstoff die höchste Konzentration. Daher liegt auch das Hauptaugenmerk in der Forschung auf dieser Verbindung. Untersuchungen zu anderen Schwefelverbindungen, wie Mercaptanen oder Carbonylsulfid, sind daher seltener.

Im Folgenden wird ein Überblick über Arbeiten zur Entschwefelung an Standardmaterialien wie Zeolithen, Aktivkohlen oder Silica-Gelen gegeben.

Untersuchungen zur adsorptiven Abtrennung von Schwefelwasserstoff an Aktivkohlen wurden in zahlreichen Beiträgen veröffentlicht. Adib et al. [12, 13] zeigen, dass die Adsorptionskapazitäten stark von der Oberflächenchemie der Kohlen abhängig sind. Durch 
die Verwendung von Imprägnierungschemikalien wie z.B. Kaliumjodid und Natriumhydroxid lassen sich diese deutlich erhöhen [14, 15, 16, 17].

Bedingt durch die vorwiegend chemisorptiven Bindungsmechanismen sind imprägnierte Adsorbentien nur teilweise oder gar nicht zu regenerieren. Diese Adsorbentien werden dementsprechend nicht in zyklisch arbeitenden Mehrbettanlagen verwendet, die in der Praxis dominieren. Eine Nische für solche Adsorbentien sind Applikationen zur Herstellung hochreiner Gase, in denen der Einsatz physikalisch wirkender Adsorbentien nicht wirtschaftlich ist, wie zum Beispiel die Bereitstellung eines Erdgases mit sehr geringen Gesamtschwefelkonzentrationen.

Zeolithe und Silica-Gele können dagegen als physisorptive Adsorbentien regeneriert werden. Für diese Adsorbentien existieren vereinzelt Daten zu Adsorptionskapazitäten und -kinetiken für verschiedene Schwefelverbindungen.

Ältere Arbeiten von Fails und von Chi behandeln die Adsorption von $\mathrm{H}_{2} \mathrm{~S}$ an verschiedenen Typ A Zeolithen als Reinstoff und aus binären Mischungen mit Kohlendioxid und ternären Mischungen mit Kohlendioxid und Methan [18, 19]. Es konnte gezeigt werden, dass die Adsorption von $\mathrm{H}_{2} \mathrm{~S}$ von weiteren in der Gasmischung enthaltenen Verbindungen beeinflusst wird. Es wurde durchweg eine signifikante Verminderung der Kapazität von $\mathrm{H}_{2} \mathrm{~S}$ bei einer Adsorption aus der Mischung im Vergleich zur Kapazität bei der Adsorption von reinem $\mathrm{H}_{2} \mathrm{~S}$ gefunden. Dies verdeutlicht den starken Einfluss konkurrierender Verbindungen auf die $\mathrm{H}_{2} \mathrm{~S}$ Adsorption.

Groninger et al. [20] berichten von detaillierten Untersuchungen zur Adsorption verschiedener Schwefelverbindungen als Reinstoff und aus Gasgemischen an einem 5A Zeolithen. Für die Reinstoffadsorption wurden im geringen Konzentrationsbereich in der Reihenfolge $\mathrm{CO}_{2}$, $\mathrm{COS}, \mathrm{H}_{2} \mathrm{~S}$ und $\mathrm{CH}_{3} \mathrm{SH}$ steigende Kapazitäten gefunden. Weiterhin konnten in einer Apparatur in Technikumsmaßstab kinetische Daten bis zu einem Prozessdruck von 100 bar bestimmt werden. Es wurden Überlegungen zur Prozessdynamik und zum Stoffaustausch angestellt. Untersuchungen zur Adsorption von $\mathrm{H}_{2} \mathrm{~S}$ aus Methan und aus Methan/Kohlendioxid zeigen Parallelen zur vorliegenden Arbeit auf. Jede zusätzliche Komponente in der Gasmischung hat einen Verlust der $\mathrm{H}_{2} \mathrm{~S}$-Kapazität des Zeolithen zur Folge und beeinflusst in starkem Maße das Durchbruchsverhalten von Schwefelwasserstoff. 
Maddox zeigt gravimetrisch bestimmte Reinstoffisothermen für $\mathrm{CO}_{2}, \mathrm{H}_{2} \mathrm{O}$ und $\mathrm{H}_{2} \mathrm{~S}$ an verschiedenen Molekularsieben bei verschiedenen Temperaturen [21] und gibt weiterführende Informationen zur Abtrennung von Schwefelverbindung in der Erdgasaufbereitung. Es werden alternative Adsorbentien, z.B. Metalloxide, zur Entschwefelung vorgestellt.

Pechler et al. [22] berichten von Untersuchungen zur simultanen Adsorption von $\mathrm{H}_{2} \mathrm{~S}$ und $\mathrm{CS}_{2}$ aus den Matrixgasen Luft und Stickstoff an zwei unterschiedlichen Zeolithen (5A, 13X). Es wurden $\mathrm{H}_{2} \mathrm{~S}$-Isothermen bei unterschiedlichen $\mathrm{CS}_{2}$-Konzentrationen gemessen. Die $\mathrm{H}_{2} \mathrm{~S}$ Kapazitäten reduzieren sich mit steigender $\mathrm{CS}_{2}-$ Konzentration in der Mischung. Dies ergibt sich ebenfalls aus der Darstellung normierter Durchbruchskurven. Es wurde eine Verdrängung des $\mathrm{H}_{2} \mathrm{~S}$ nachgewiesen. Mit Hilfe zweier Modelle, einem kinetischen LDF-Ansatz und einem Diffusionsmodell nach dem 2. Fick`schen Gesetz, ließen sich die gemessenen Durchbrüche mit sehr guter Genauigkeit beschreiben. Es werden jedoch keine Zahlenwerte für die Modellparameter angegeben. Die Adsorption am $13 \times$ Zeolith aus dem Trägergas Luft weist für auf eine Oxidation des $\mathrm{H}_{2} \mathrm{~S}$ mit dem Luft-Sauerstoff zu elementaren Schwefel hin.

Sakano et al. [23] zeigen Reinstoffuntersuchungen für Methylmercaptan, Kohlendioxid, Wasser und weiteren Verbindungen für die Adsorption an einem $5 \mathrm{~A}$ Zeolith. Es wurde eine starke Abhängigkeit der Adsorptionskapazitäten von den Polaritäten der Adsorptive gefunden. In Durchbruchskurvenmessungen in einer Festbettversuchsanlage konnte für die Adsorption von Methylmercaptan eine starke Beeinflussung durch zusätzlich in der Gasmischung enthaltenes Wasser nachgewiesen werden. Adsorbierendes Wasser verdrängt einen Großteil bereits gebundener $\mathrm{CH}_{3} \mathrm{SH}-$ Moleküle aus dem Porensystem des verwendeten Zeolithen.

Weber et al. $[24,25]$ untersuchten in mehreren Beiträgen die Adsorption von Ethylmercaptan an NaX-Zeolithen sowohl als Reinstoff als auch als binäre Mischung mit Kohlenwasserstoffen. Je nach Polarität der Kohlenwasserstoff-Verbindung (n-Heptan, Toluol) konnten unterschiedlich starke Beeinflussungen der Adsorption von Ethylmercaptan ausgemacht werden. Es wurden keine kinetischen Betrachtungen angestellt.

Sarbak berichtet von modifizierten NaX-Zeolithen zur katalytischen Umsetzung von Ethylmercaptan [26]. Ryzhikov et al. [27] untersuchten die Adsorption von Methylmercaptan und Carbonylsulfid im Spurenbereich an diversen Zeolithen der Typen X und Y. In Durchbruchskurvenmessungen konnte eine starke Abhängigkeit der Adsorption von Methylmercaptan 
vom Metallkation der untersuchten Zeolithe nachgewiesen werden. Die Durchbruchskurven wurden nach einem Ansatz von Yoon und Nelson [28] modelliert.

Eine ältere Arbeit von Tanada et al. thematisiert die Reinstoffadsorption von Schwefelwasserstoff an einem 5A Zeolith und einem Kohlenstoffmolekularsieb [29].

Gleichgewichtsdaten oder Durchbruchskurven der aufgeführten Schwefelverbindungen an Silica-Gelen sind kaum veröffentlicht. Zhou Li et al. untersuchten das Durchbruchsverhalten von Schwefelwasserstoff im Spurenbereich an einem imprägnierten Silica-Gel in einer Pressure Swing Adsorption Versuchsanlage [30]. Es wurden gute Reinigungsleistungen in kurzen Adsorptionszyklen bei verschiedenen Drücken nachgewiesen.

Zusammenfassend lässt sich sagen, dass lediglich für die Verbindung Schwefelwasserstoff zahlreiche Daten zur Adsorption vorhanden sind. Für andere im Rahmen der Arbeit betrachtete Schwefelverbindungen ist die Datenlage lückenhaft.

Um Messdaten zur Auslegung großtechnischer Anlagen verwenden zu können, müssen die untersuchten Stoffsysteme denen in der Praxis möglichst ähnlich sein. Dies ist häufig nicht der Fall. Bei der Vermessung von Gleichgewichten wird oft ein sehr weiter Konzentrationsbereich gewählt, während im technisch oft interessanteren Spurenbereich nur wenige Daten vorliegen. Studien zur Adsorptionskinetik der Schwefelverbindungen in geringen Konzentrationsbereichen sind rar. Um die Stoffsysteme in der adsorptiven Erdgasentschwefelung besser $\mathrm{zu}$ verstehen, fehlen detaillierte dynamische Adsorptionsexperimente zur Abtrennung der Schwefelverbindungen bis in den Spurenbereich $(\leq 2 \%$ ) in Gegenwart von Methan als der Hauptkomponente des Erdgases sowie von Wasser und $\mathrm{CO}_{2}$ als den wichtigsten Nebenbestandteilen.

\subsection{Motivation und Zielstellung der Arbeit}

Die Bereitstellung wirtschaftlicher Adsorptionsverfahren zur Gasaufbereitung erfordert eine stetige Optimierung der Trennprozesse und ein besseres Verständnis der stattfindenden Adsorptionsmechanismen. Eine umfassende Betrachtung der in einer kontinuierlich durchströmten Festbettschüttung ablaufenden Vorgänge ist daher von hohem Interesse für die Industrie. Zur Auslegung industrieller Adsorptionsprozesse sind zum einen genaue Gleichgewichtsdaten im Spurenbereich notwendig, zum anderen wird ein Transportmodell benötigt, um das dynamische Verhalten des Prozesses zu beschreiben. 
Die vorliegende Arbeit soll einen Beitrag dazu leisten, die Vorgänge bei der Gasentschwefelung im Adsorberfestbett besser zu verstehen. Dazu soll mit einer eigens am Lehrstuhl aufgebauten Festbett-Versuchsanlage die Adsorption praxisähnlicher Gasmischungen mit Schwefelverbindungen an industriell eingesetzten Adsorbentien untersucht werden. Neben der Vermessung binärer Stoffsysteme (1 Adsorptiv im Trägergas) sollten durch Zugabe weiterer Adsorptivmoleküle ternäre Systeme (2 Adsorptive im Trägergas) erzeugt werden, um gezielt Konkurrenzsituationen auf der Adsorbensoberfläche zu schaffen. Auf diese Weise kann der Einfluss der Koadsorption anderer Verbindungen auf die Adsorption der Schwefelverbindungen beschrieben werden.

Die Vermessung der Gleichgewichte liefert Daten für die Bestimmung der Kapazität der Adsorbentien im Prozess. Die kinetischen Versuchsdaten der Adsorption im Festbett können mathematisch modelliert und dynamisch simuliert werden. Dadurch lassen sich die kinetischen Parameter des Transportmodells extrahieren. Dies ermöglicht ein vertieftes Verständnis der Prozessdynamik und bildet die Grundlage für eine bessere Dimensionierung einer Gasaufbereitungsanlage in der Praxis. 


\section{Theoretische Grundlagen}

Im folgenden Kapitel werden grundlegende Begriffe zur Gasphasenadsorption erläutert. Insbesondere die Prozessdynamik, Adsorptionskinetik und der Stofftransport werden detailliert betrachtet. Alle im Rahmen des experimentellen Teils der Arbeit verwendeten Adsorbentien und Adsorptive werden vorgestellt. Zuletzt folgt eine Beschreibung des verwendeten physikalischen Modells zur mathematischen Beschreibung dynamischer Adsorptionsprozesse.

\subsection{Begriffsdefinitionen und Grundlagen der Gasphasenadsorption}

Die Adsorption bezeichnet die Anlagerung von Molekülen aus einer fluiden Phase an einer festen Phase. Diese feste Phase wird als Adsorbens bezeichnet. Ein in der freien fluiden Phase befindliches Molekül, das im Laufe des Adsorptionsprozesses Wechselwirkungen mit der Oberfläche des Adsorbens eingeht, ist das Adsorptiv. Im gebundenen oder angelagerten Zustand wird es als Adsorpt bezeichnet. Der Komplex aus Adsorbens und Adsorpt bildet das Adsorbat oder die Adsorbatphase. Der umgekehrte Vorgang, das Lösen des Adsorpts von der Oberfläche und der damit verbundene Übergang in die fluide Phase, werden als Desorption bezeichnet. Abbildung 2 erläutert die beschriebenen Phänomene. 


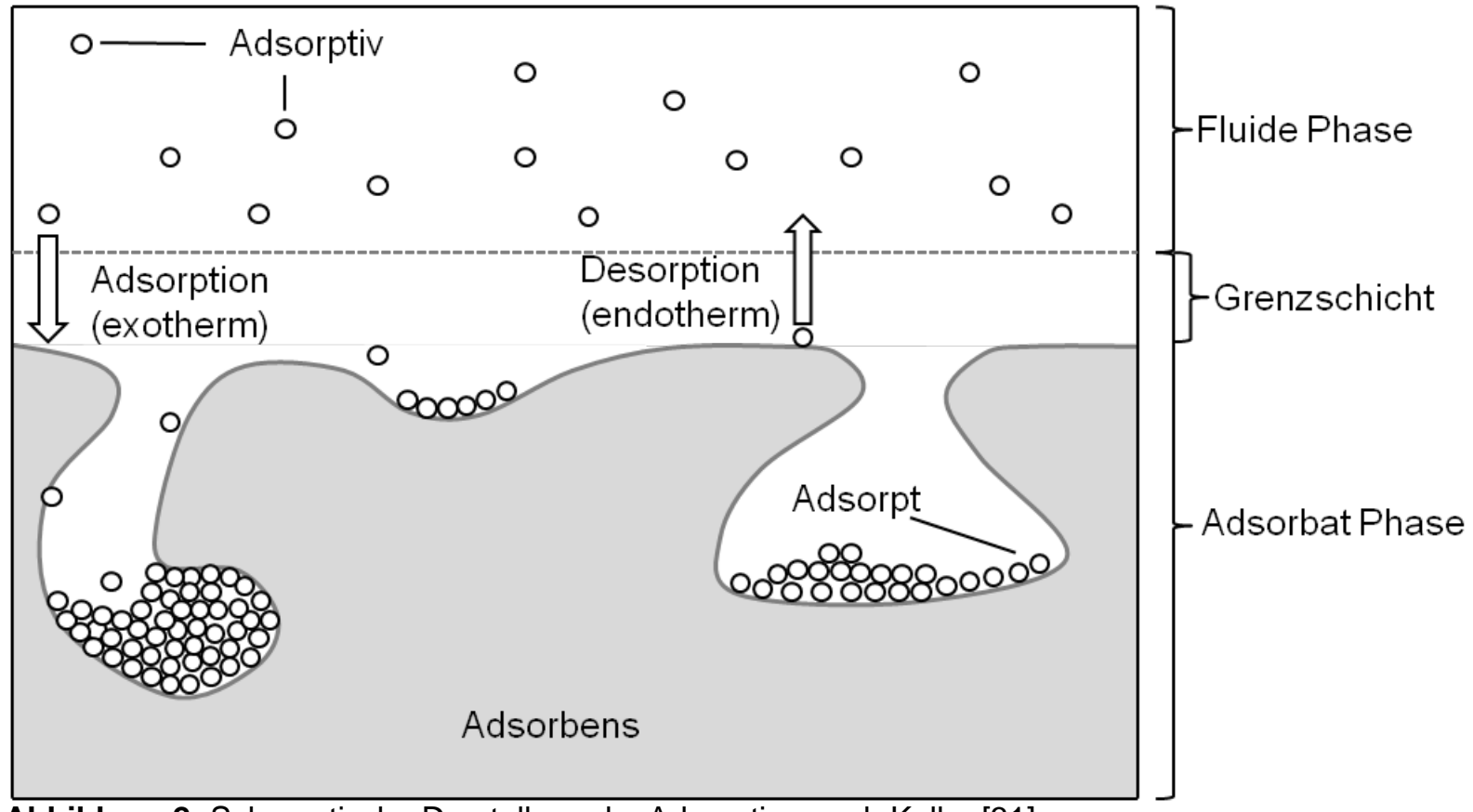

Abbildung 2: Schematische Darstellung der Adsorption nach Keller [31]

Moleküle können auf unterschiedliche Art und Weise mit der Oberfläche des Feststoffs wechselwirken. Anhand der Adsorptionsenthalpie lässt sich die Adsorption in zwei verschiedene Mechanismen unterteilen.

Bei der Physisorption treten geringe Adsorptionsenthalpien im Bereich der 1,5-fachen Verdampfungsenthalpie des Adsorptivs auf [32]. Es kommt zu van der Waals- oder elektrostatischen Wechselwirkungen zwischen Adsorptiv und Adsorbens. Solche Bindungen sind durch moderate Zustandsänderungen wie z. B. Temperaturerhöhung oder Druckabsenkung wieder zu lösen. Der Begriff der Chemisorption beschreibt eine stärkere Bindung von Molekülen. Es kommt zu Elektronenübergängen und einer chemischen Reaktion zwischen Adsorptiv und Adsorbensoberfläche. Die vorliegende Bindungsenergie ist mit dem 2-3fachen der Verdampfungsenthalpie des Adsorptivs deutlich größer als bei der Physisorption. Diese Art der Bindung lässt sich unter wirtschaftlichen Bedingungen nur teilweise oder gar nicht lösen. Für die im Rahmen dieser Arbeit untersuchten Stoffsysteme stellt die Physisorption den bedeutenden Wechselwirkungsmechanismus zwischen Adsorbens und Adsorptiv dar.

\subsubsection{Thermodynamik der Adsorption}

Bei einem thermischen Trennverfahren kommt es unter konstanten Prozessbedingungen (Druck, Temperatur, Konzentration) zu einem Zustand, bei dem alle Triebkräfte für einen 
Stoffübergang zum Erliegen kommen. Die beteiligten Phasen stehen dann im Gleichgewicht miteinander.

Ein bei der Adsorption herrschendes Gleichgewicht des Zweiphasensystems Feststoff $\Leftrightarrow$ Fluid lässt sich anhand von Adsorptionsisothermen beschreiben. Die Adsorptionsisotherme beschreibt den direkten Zusammenhang zwischen der Konzentration oder dem Partialdruck einer in der fluiden Phase befindlichen Komponente und ihrer Anreicherung an der festen Phase bei konstanter Temperatur.

Im Folgenden seien die für diese Arbeit relevanten Isothermentypen sowie die diesen zugrunde liegenden Annahmen aufgeführt.

\section{Henry-Isotherme}

Die Isothermengleichung nach Henry beschreibt einen linearen Zusammenhang zwischen der Konzentration eines Adsorptivs in der fluiden Phase und ihrer Beladung in der festen Phase:

$$
X_{G l}=k_{H} \cdot y
$$

Der Gleichung liegt die Annahme zugrunde, dass es unendlich viele, energetisch gleichwertige Adsorptionsplätze gibt, die für Adsorptiv-Moleküle unbegrenzt erreichbar sind. Wechselwirkungen zwischen adsorbierten Molekülen treten nicht auf. Die HenryIsothermengleichung findet Verwendung bei der Beschreibung von Adsorptionsgleichgewichten in sehr geringen Konzentrations- und Beladungsbereichen. Abbildung 3 zeigt beispielhaft eine Henry-Isotherme.

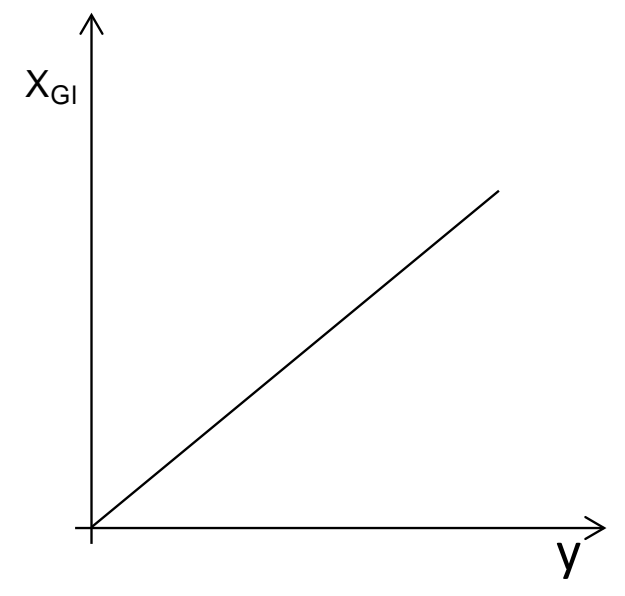

Abbildung 3: Henry-Isotherme 


\section{Freundlich-Isotherme}

Eine weitere Gleichung zur Beschreibung eines Adsorptionsgleichgewichtes geht auf Freundlich zurück [33]. Die zweiparametrige Potenzfunktion lautet:

$$
X_{G l}=k_{F} \cdot y^{n}
$$

Es handelt sich um eine rein empirische Gleichung, die thermodynamisch nicht begründet ist. Für den Grenzfall $n=1$ geht sie in die Henry-Gleichung über. Abbildung 4 zeigt beispielhaft drei Freundlich-Isothermen für verschiedene Exponenten. Je nach Größe des Exponenten kann die Freundlich-Isotherme eine konvexe oder eine konkave Form annehmen.

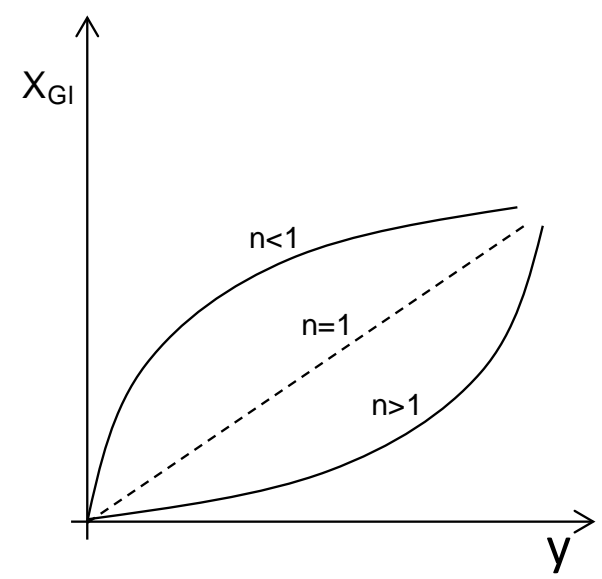

Abbildung 4: Verschiedene Freundlich-Isothermen

\section{Langmuir-Isotherme}

Die Isothermengleichung nach Langmuir [34] stellt wie die Freundlich-Gleichung eine zweiparametrige Gleichung dar. Langmuir begründet seine Gleichung jedoch thermodynamisch. Die Gleichung hat die Form

$$
X_{G l}=X_{\text {mon }} \frac{y \cdot b}{1+y \cdot b}
$$

Alle Adsorptionsplätze werden als energetisch gleichwertig betrachtet und können von Adsorptiv-Molekülen besetzt werden. Es finden keine Wechselwirkungen zwischen adsorbierten Molekülen statt. Die fluide Phase wird als ideal betrachtet. Im Gegensatz zur HenryGleichung ist die Anzahl der Adsorptionsplätze begrenzt. Es findet keine mehrschichtige Adsorption statt, sondern es kommt ausschließlich zu einer monomolekularen Bedeckung 
der Adsorbensoberfläche. Abbildung 5 zeigt beispielhaft eine Langmuir-Isotherme. Ein Charakteristikum der Langmuir-Isotherme ist der Verlauf gegen einen Beladungsgrenzwert.

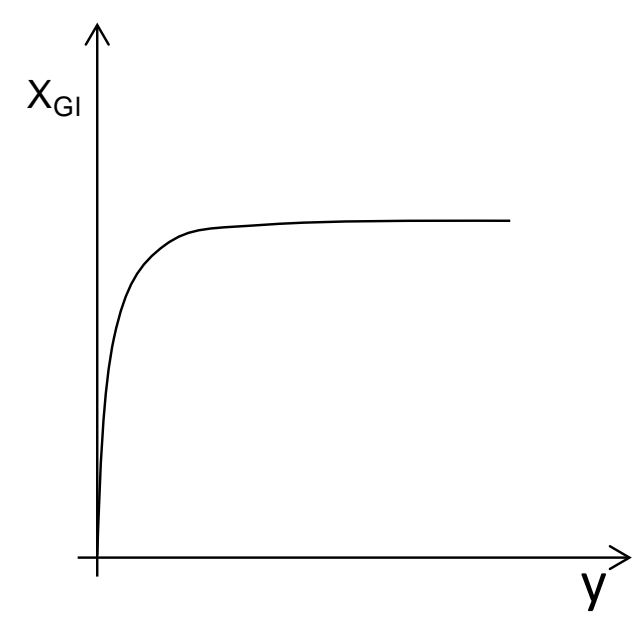

Abbildung 5: Langmuir-Isotherme

Neben den hier beschriebenen Isothermen-Typen gibt es zahlreiche weitere Modelle. Da sich jedoch alle experimentell betrachteten Stoffsysteme in sehr guter Näherung mit Hilfe der oben aufgeführten Gleichungen beschreiben ließen, sei an dieser Stelle auf die relevanten Monographien [32, 35, 36, 37] verwiesen.

\section{Gleichgewichtsbeziehungen für die Adsorption mehrerer Komponenten}

Im Hinblick auf die im Rahmen dieser Arbeit untersuchten ternären Stoffsysteme, bei denen es zu einer Adsorption mehrerer Adsorptive kommt, lassen sich weiterführende Modelle zur Beschreibung und Vorhersage von Mehrkomponentengleichgewichten verwenden. Es kann zwischen verschiedenen Ansätzen unterschieden werden [38]. Im Folgenden wird auf den semiempirischen Ansatz nach Markham und Benton [39] und die auf der Gleichgewichtsthermodynamik fußende Theorie der ideal adsorbierten Lösung (IAST, Ideal Adsorbed Solution Theory) nach Myers und Prausnitz [40] eingegangen. Diese beiden Ansätze wurden auf die experimentellen Daten angewendet und sind daher für die spätere Diskussion von Bedeutung.

\section{Erweiterter Langmuir-Ansatz nach Markham und Benton}

Markham und Benton [39] erweiterten die Langmuir-Isothermengleichung auf mehrere Komponenten. Für den hier relevanten Fall, dass zwei Verbindungen adsorbieren, ergibt sich die Lösung: 


$$
\begin{aligned}
& x_{1}=\frac{x_{m o n, 1} \cdot b_{1} \cdot y_{1}}{1+b_{1} \cdot y_{1}+b_{2} \cdot y_{2}} \\
& X_{2}=\frac{x_{m o n, 2} \cdot b_{2} \cdot y_{2}}{1+b_{1} \cdot y_{1}+b_{2} \cdot y_{2}}
\end{aligned}
$$

Die Gleichgewichtsbeschreibung oder -vorhersage eines Systems mit zwei Adsorptiven ist damit anhand der Einkomponenten-Gleichgewichte möglich, wenn diese beide durch die Langmuir-Gleichung beschrieben werden können. Streng genommen ist dieser Ansatz nicht thermodynamisch konsistent, da nach Langmuir die monomolekulare Bedeckung für jede Komponente gleich sein sollte. Der Ansatz liefert jedoch im Bereich geringer Konzentrationen trotzdem gute Ergebnisse [38].

\section{Theorie der ideal adsorbierten Lösung (IAST)}

Die Theorie der ideal adsorbierten Lösung (Ideal Adsorbed Solution-Theory) nach Myers und Prausnitz [40] baut auf dem thermodynamischen Gleichgewicht zwischen adsorbierter Phase und fluider Phase auf.

Die freie Enthalpie der Adsorbatphase mit mehreren Komponenten ist eine Funktion der Temperatur, des Spreizdrucks und der Zusammensetzung $n_{i}$ :

$$
G=G\left(T, \pi, n_{i}\right)
$$

Bei dieser Beschreibung ist $\pi$ der Spreizdruck, der als Änderung der spezifischen Energie der Oberfläche (Grenzflächenspannung) infolge der Adsorption betrachtet werden kann. Das totale Differential der freien Enthalpie G lässt sich zu Gleichung 2.7 formulieren:

$$
d G=\left.\frac{\partial G}{\partial T}\right|_{\pi, n_{i}} d T+\left.\frac{\partial G}{\partial \pi}\right|_{T, n_{i}} d \pi+\left.\sum \frac{\partial G}{\partial n_{i}}\right|_{T, \pi, n_{j(i \neq j)}} d n_{i}
$$

Diese Gleichung lässt sich mit der Entropie $S$, einer spezifischen Oberfläche $A$ und dem chemischen Potential der Adsorbatphase darstellen als:

$$
d G=-S d T+A d \pi+\sum_{i} \mu_{i} d n_{i}
$$

Unter Verwendung des Euler'schen Theorems, welches besagt, dass jede extensive Größe in einer Mischung die Summe der Produkte der partiellen (molaren) Größen mit ihrem Anteil in der Mischung ist, ergibt sich 


$$
\begin{gathered}
G=\sum_{i} \mu_{i} \cdot n_{i} \\
d G=\sum_{i} \mu_{i} \cdot d n_{i}+\sum_{i} n_{i} \cdot d \mu_{i}
\end{gathered}
$$

Ersetzt man den differentiellen Ausdruck für $d G$ in Gleichung 2.8 ergibt sich die GibbsDuhem-Gleichung der Form:

$$
S d T-A d \pi+\sum_{i} n_{i} \cdot d \mu_{i}=0
$$

Bedingung für ein thermodynamisches Gleichgewicht ist die Gleichheit des chemischen Potenzials einer Komponente $\mathrm{i}$ in der Adsorbatphase und der fluiden Phase:

$$
\mu_{i, A d s}=\mu_{i, F l} \text { bzw. } d \mu_{i, A d s}=d \mu_{i, F I}
$$

Das chemische Potential der fluiden Phase lässt sich unter Annahme eines idealen Gases schreiben zu:

$$
\mu_{i, F I}=\mu_{i}^{0}(T)+R \cdot T \cdot \ln (y \cdot p)
$$

Das chemische Potential der Adsorbatphase lässt sich unter Berücksichtigung von Aktivitätskoeffizienten darstellen als:

$$
\mu_{i, A d s}=R \cdot T \cdot \ln \left(Y_{i} x_{i}\right)+\mu_{i}^{0}
$$

Hierbei ist $X_{i}$ die Zusammensetzung der Adsorbatphase und $\mu_{i}^{0}$ das chemische Potential einer Komponente, wenn sie alleine die adsorbierte Phase bildet. Dieses ist eine Funktion der Temperatur und des Spreizdruckes:

$$
\mu_{i}^{0}=\mu_{i}^{0}(T, \pi)
$$

Für eine reine Komponente gilt mit dem Dampfdruck $p_{i}^{0}(\pi)$ für das chemische Potential:

$$
\mu_{i}^{0}(T, \pi)=\mu_{i}^{0}(T)+R \cdot T \cdot \ln \left(p_{i}^{0}(\pi)\right)
$$

$p_{i}^{0}(\pi)$ entspricht dabei einem hypothetischen Gleichgewichts-Dampfdruck der Komponente i, der zum Spreizdruck $\pi$ in der Adsorbatphase führt. $\mu_{i}^{0}(T)$ ist das chemische Potential der Komponente i als perfektes Gas bei Standarddruck (1 bar).

Das chemische Potential der Adsorbatphase ergibt sich damit aus Gleichungen 2.14 und $2.16 \mathrm{zu}$ : 


$$
\mu_{i, A d s}\left(T, \pi, x_{i}\right)=\mu_{i}^{0}(T)+R \cdot T \cdot \ln \left(p_{i}^{0}(\pi)\right)+R \cdot T \cdot \ln \left(\gamma_{i} x_{i}\right)
$$

Setzt man Gleichungen 2.13 und 2.17 gleich, erhält man analog zur Beschreibung eines Dampf-Flüssigkeit-Gleichgewichts nach dem erweiterten Raoult'schen Gesetz das AdsorbatGas-Gleichgewicht der Form:

$$
y_{i} \cdot p=\gamma_{i} \cdot x_{i} \cdot p_{i}^{0}(\pi)
$$

Für eine ideal adsorbierte Lösung reduziert sich Gleichung 2.18 durch Wegfall des Aktivitätskoeffizienten zu

$$
y_{i} \cdot p=x_{i} \cdot p_{i}^{0}(\pi)
$$

Einen Ausdruck, der einen Zusammenhang zwischen dem hypothetischen GleichgewichtsDampfdruck $p_{i}^{0}(\pi)$ und dem Spreizdruck $\pi$ herstellt, liefert die Gibbs-Duhem-Gleichung (Gleichung 2.11). Für die Betrachtung eines einzelnen Adsorptivs bei konstanter Temperatur erhält man unter Berücksichtigung des chemischen Potenzials (Gleichung 2.16) die Gibbs'sche Adsorptionsisotherme der Form:

$$
A \cdot d \pi=n_{i}^{0} \cdot R \cdot T \cdot d\left[\ln \left(p_{i}^{0}(\pi)\right)\right]
$$

In einer ideal adsorbierten Lösung ist der Spreizdruck jeder Komponente in der Mischung gleich dem Spreizdruck der adsorbierten reinen Komponenten. Außerdem ergibt sich die Zusammensetzung der Mischung so, dass die Komponenten denselben Spreizdruck haben wie die Mischung. Es gilt:

$$
\Pi_{1}^{0}=\pi_{2}^{0}=\ldots \ldots=\pi=\text { const }
$$

Damit liefert Gleichung 2.22 einen Ausdruck, der einen Zusammenhang zwischen den beiden Größen $p_{i}^{0}$ und $\pi$ beschreibt. Beispielhaft für die Adsorption von zwei Komponenten ergibt sich:

$$
\frac{A \cdot \pi}{R \cdot T}=\int_{0}^{p_{1}^{0}} \frac{n_{1}^{0}}{p_{1}^{0}} \cdot d p_{1}^{0}=\int_{0}^{p_{2}^{0}} \frac{n_{2}^{0}}{p_{2}^{0}} \cdot d p_{2}^{0}
$$

$n_{i}^{0}$ stellt hierbei die molare Beladung der Komponente i für den Fall der EinkomponentenAdsorption dar. Sie kann durch die experimentell bestimmte Isothermen-Gleichung ausgedrückt werden. Die Stoffmengenanteile der Adsorptive in Adsorbat- und fluider Phase müssen die Bedingungen 


$$
\begin{aligned}
& \sum_{i=1}^{N} y_{i}=1 \\
& \sum_{i=1}^{N} x_{i}=1
\end{aligned}
$$

erfüllen.

Unter der Annahme, dass das Verhältnis der benetzten Oberflächen im Gemisch $\left(A_{i}\right)$ und im Reinstoff ( $A_{\text {ges }}$, die insgesamt benetzte Oberfläche) bei gleicher Temperatur und gleichem Spreizdruck dem Verhältnis der jeweils absorbierten Stoffmengen entspricht, gilt:

$$
\begin{aligned}
& \frac{n_{i}}{n_{i}^{0}}=\frac{A_{i}}{A} \\
& \sum_{i} \frac{n_{i}}{n_{i}^{0}}=1
\end{aligned}
$$

Für den oben gegebenen Fall, dass zwei Komponenten adsorbieren, lassen sich die adsorbierten Stoffmengen in diesem Fall mit Gleichung 2.27 beschreiben (Lewis-Regel [41]), wenn man durch die adsorbierte Gesamtstoffmenge dividiert:

$$
\frac{x_{1}}{n_{1}^{0}}+\frac{x_{2}}{n_{2}^{0}}=\frac{1}{n_{\text {ges }}}
$$

Somit ist die adsorbierte Gesamtstoffmenge $n_{\text {ges }}$ rechnerisch zugänglich und über die Anteile $X_{i}$ lassen sich ebenfalls die adsorbierten Mengen $n_{i}$ ermitteln.

Für bekannte Isothermengleichungen gibt es analytische Lösungen des Integrals (Gleichung 2.22). Im Falle der Langmuir-Gleichung lässt sich der Zusammenhang zwischen Spreizdruck und hypothetischem Dampfdruck $p_{i}^{0}$ über den Ausdruck

$$
\frac{\pi \cdot A}{R \cdot T}=n_{m o n, i} \cdot \ln \left(1+b_{i} \cdot p_{i}^{o}\right)
$$

beschreiben. Für ein Henry-Gleichgewicht ergibt sich:

$$
\frac{\pi \cdot A}{R \cdot T}=k_{H, i} \cdot p_{i}^{0}=n_{i}^{0}
$$

Für ein Stoffsystem mit 2 adsorbierbaren Komponenten und bekannter Zusammensetzung der Gasphase müssen fünf unbekannte Größen bestimmt werden: 


$$
p_{1}^{0}, p_{2}^{0}, x_{1}, x_{2}, n_{\text {ges }}
$$

Unter Verwendung der angegebenen Gleichungen sind diese Unbekannten rechnerisch zugänglich.

Die Anwendung der IAS-Theorie und der erweiterten Langmuir-Gleichung nach Markham und Benton auf die im Rahmen der Arbeit untersuchten Gleichgewichte findet sich in Abschnitt 4.4.4.

Darüber hinaus finden sich in der Literatur sowohl weitere thermodynamische als auch empirische Modelle zur Beschreibung und Vorhersage von Mehrkomponentengleichgewichten. Als Erweiterung der IAS-Theorie ist die Theorie der real adsorbierten Lösung zu sehen [42]. Nichtidealitäten können durch die Verwendung von Aktivitätskoeffizienten berücksichtigt werden. Diese lassen sich auf Grundlage verschiedener Ansätze (z. B. Wilson-Ansatz [43]) ermitteln. Eine weitere Theorie, die ebenfalls Aktivitätskoeffizienten berücksichtigt, ist die Theorie der Leerstellenlösung (Vacancy Solution-Theory) [44]. Weitere bekannte empirische Modelle sind beispielsweise die Ansätze von Hill [45] und Gonzales-Holland [46].

Einen vertieften Einblick in diese Thematik bieten die relevanten Monographien [32, 35, 36, $37,38]$.

\subsubsection{Dynamik durchströmter Schüttungen}

Die Dynamik einer durchströmten Schüttung ist über die so genannte Durchbruchskurve zugänglich. Die Durchbruchskurve beschreibt die zeitliche Veränderung der Gaszusammensetzung einer kontinuierlich durchströmten Schüttung gemessen am Adsorberaustritt hinter der Schüttung. In der Schüttung selber bilden sich bei richtiger Dimensionierung des Adsorbers und konstanten Strömungs- und Konzentrationsbedingungen gekoppelte Konzentrations- (Partialdruck-) und Beladungsprofile aus. Diese Profile oder Fronten in der Schüttung sind ebenfalls experimentell bestimmbar, jedoch nur unter hohem apparativen Aufwand, da man hierfür Kenntnis über die Zusammensetzung der fluiden Phase an zahlreichen Stellen innerhalb der Schüttung haben muss. Im Gegensatz dazu erfordert die Messung der Durchbruchskurve lediglich die Analyse des aus der Schüttung austretenden Produktgases. Die Form der Fronten und der Durchbruchskurve ist von entscheidender Relevanz für den Betrieb einer Adsorptionsanlage [47]. Wichtige Parameter wie die Taktung zwischen Adsorptions- und Desorptionstakt oder der Bettausnutzungsgrad hängen vom Durchbruchsverhalten der beteiligten Adsorptive ab. 
Abbildung 6 zeigt beispielhaft das Konzentrationsprofil in einer Schüttung für den einfachen Fall der isothermen Einkomponenten-Adsorption.

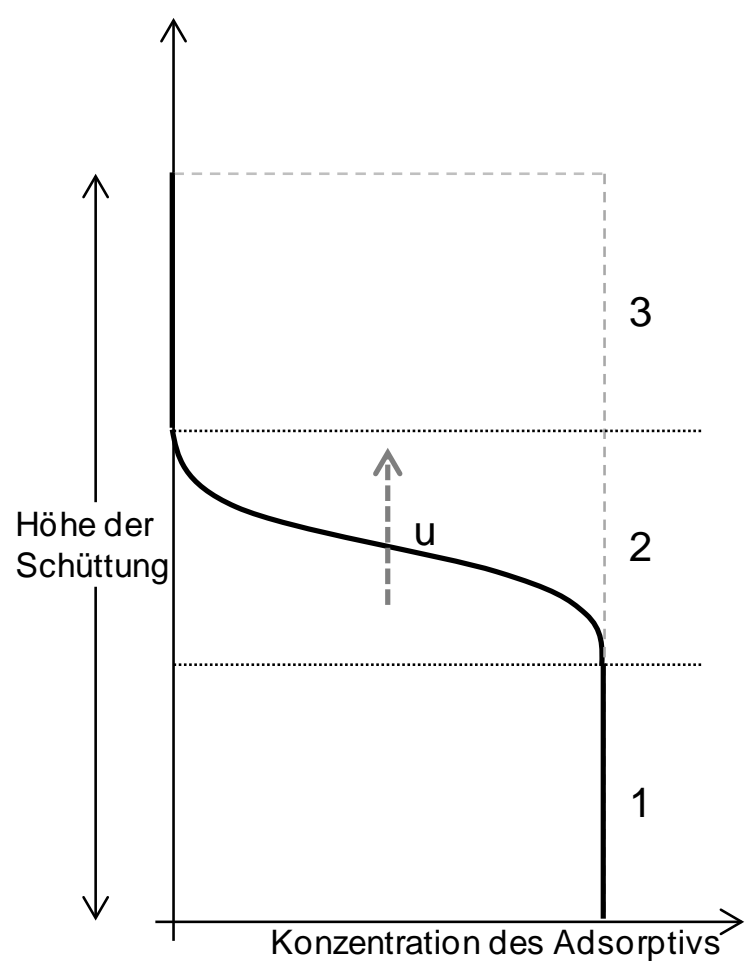

Abbildung 6: Konzentrationsprofil in einer durchströmten Schüttung

Es zeigt sich bei konstanten Prozessbedingungen (Volumenstrom, Konzentration, Temperatur, Druck) die Ausbildung von drei Zonen:

Zone 1: In dieser Zone ist das Adsorbens bereits vollständig beladen. Es herrscht stoffliches Gleichgewicht. Die Adsorptivkonzentration in diesem Bereich entspricht der des Rohgases. Mit zunehmender Adsorptionsdauer wird dieser Bereich einen immer größeren Teil der Schüttung einnehmen. In einem Adsorptionsprozess befindet sich zum Ende des Adsorptionszyklus idealerweise das gesamte Bett in diesem Zustand. Ein geringer Anteil der Schüttung bleibt jedoch auf Grund der Breite der Massentransferzone (Zone 2) ungenutzt (LUB= Length of Unused Bed [38]). Je geringer dieser ungenutzte Anteil der Schüttung ist, desto besser ist der Bettausnutzungsgrad und demzufolge des Wirtschaftlichkeit des Prozesses.

Zone 2: In diesem Bereich findet die Adsorption statt. Der Bereich wird als Massentransferzone bezeichnet. Er wandert mit zunehmender Dauer der Adsorption durch die Schüttung. Die Breite der Zone bzw. die Steigung und die Form der Konzentrationsfront sind beeinflusst durch die vorherrschenden Strömungszustände, das Adsorptionsgleichgewicht und die Adsorptionskinetik. 
Zone 3: In diesem Bereich ist das Adsorbens noch unbeladen. Die Adsorptivkonzentration ist gleich null. Zu Beginn der Adsorption liegt dieser Zustand in der gesamten Schüttung vor. Das Adsorptiv ist noch nicht bis in diese Zone vorgedrungen, da es in der Massentransferzone komplett aus der fluiden Phase abgetrennt wird.

Die gezeigte Darstellung ist als Momentaufnahme zu einem bestimmten Zeitpunkt zu verstehen. Sowohl die gekoppelten Beladungs- und Konzentrationsprofile in der Schüttung als auch die sich aus diesen ergebende Durchbruchskurve, die am Adsorberaustritt gemessen werden kann, unterliegen während der Adsorption verschiedenen Einflussfaktoren (vgl. Abschnitt 2.1.3).

\subsubsection{Kinetik der Adsorption und Stofftransportphänomene}

Prägt man einem im thermodynamischen Gleichgewicht befindlichen Adsorbens-AdsorptivSystem von außen ein Ungleichgewicht auf, verschiebt sich die Lage des Gleichgewichts. Die Geschwindigkeit, mit der das System versucht, dieses Ungleichgewicht auszugleichen, wird durch die Adsorptionskinetik beschrieben. Sie ist bestimmt durch die vorherrschenden Widerstände für den Stoff- und Energietransport.

Der Stofftransport der Adsorption in einer kontinuierlich durchströmten Schüttung lässt sich in verschiedene Schritte aufteilen. Zunächst gelangen die Adsorptivmoleküle durch Konvektion und freie Diffusion aus der fluiden Phase durch die Grenzschicht der Adsorbenskörner an die äußere Oberfläche. Danach erfolgt der Stofftransport im Porensystem der Adsorbentien. Dieser lässt sich, je nach Porenart, wiederum in verschiedene Diffusionsmechanismen unterteilen. Als letzter Stofftransportmechanismus folgt die eigentliche Adsorption mit der energetischen Wechselwirkung zwischen Adsorptiv und Adsorbens [47].

Den entscheidenden, geschwindigkeitsbestimmenden Anteil am gesamten Adsorptionsprozess hat unter der Voraussetzung korrekt gewählter Strömungszustände und Adsorbergeometrien der Stofftransport der Adsorptivmoleküle im Porensystem des Adsorbens. Die Adsorption selber läuft sehr schnell ab und ist in einer kinetischen Betrachtung für die meisten Systeme zu vernachlässigen. Dies gilt bei hinreichend großer Strömungsgeschwindigkeit ebenso für den Transportwiderstand durch den Grenzfilm [47].

Der Stofftransport im Porensystem ist sowohl vom Adsorbens als auch vom Adsorptiv abhängig. Je nach System gibt es vier Transportmechanismen in Gasphasenanwendungen bei geringen Drücken, die analog zum Fick’schen Gesetz der Form 


$$
\dot{m}=-D \cdot A_{\text {spez }} \cdot \frac{\partial Y}{\partial r}
$$

formuliert werden können:

i. Freie Porendiffusion

ii. Knudsen-Diffusion

iii. Oberflächendiffusion

iv. Interkristalline Diffusion

Je nach vorherrschendem Mechanismus kann eine spezifische Fläche und die Potenzialgröße $Y$ als Triebkraft (z. B. Konzentrationsgradient, Beladungsgradient) der Diffusion für die Beschreibung des Transportmassenstroms herangezogen werden. Abhängig vom Porensystem des Adsorbens und vom Adsorptiv findet eine Überlagerung der verschiedenen Diffusionsmechanismen statt.

Bei geringen Betriebsdrücken und geringen Beladungen im Bereich der monomolekularen Bedeckung sind Freie Porendiffusion und Knudsen-Diffusion die beiden Transportphänomene, denen die wichtigste Bedeutung zukommt. Als Kriterium zur Abschätzung des dominanten Stofftransportwiderstandes zwischen diesen kann die Knudsen-Zahl herangezogen werden [47]. Sie ist definiert zu:

$$
K n=\frac{\lambda_{F}}{d_{P}}
$$

Nach Gleichung 2.31 ist die Knudsen-Zahl abhängig vom Adsorptivmolekül und von der Porenart des Adsorbens.

Liegt ein mikroporöses Adsorbens mit deutlich kleineren Poren als der mittleren freien Weglänge des Adsorptivs vor, ist die Wahrscheinlichkeit für Stöße zwischen Porenwand und Adsorptiv groß. Der Stofftransport ist in diesem Fall durch die Diffusion nach Knudsen dominiert. Der entsprechende Diffusionskoeffizient lässt sich mit Gleichung 2.32 beschreiben:

$$
D_{K n}=\frac{4}{3} \cdot \frac{d_{P o r e}}{\mu_{P, K n}} \cdot \sqrt{\frac{R \cdot T}{2 \pi \cdot M_{A}}}
$$

Der temperaturveränderliche Knudsen-Diffusionskoeffizient hängt von den Moleküleigenschaften und vom Durchmesser der Poren ab. Der zusätzlich enthaltene Tortuositätsfaktor $\mu_{P, K n}$ erfasst die Widerstände des Stofftransports infolge der starken Verengung, Umlenkung und Verwundenheit des Porensystems.

Als Grenze zwischen Freier und Knudsen-Diffusion finden sich in der Literatur KnudsenZahlen $>10$. Ergeben sich kleine Knudsen-Zahlen $(<10)$ für eine Adsorbens-Adsorptiv- 
Paarung wird der Stofftransport durch Stöße zwischen Adsorptivmolekülen dominiert. In diesem Fall ist die Freie Porendiffusion der dominierende Mechanismus. Der Diffusionskoeffizient für die Freie Porendiffusion errechnet sich aus dem molekularen Diffusionskoeffizienten korrigiert um die zusätzlichen Widerstände auf Grund des Porensystems

$$
D_{\text {Diff }}=\frac{D_{1,2}}{\mu_{P, \text { Diff }}}
$$

Bei dieser Formulierung beschreibt $D_{1,2}$ für ein binäres Gasgemisch die Diffusion der einen Komponente in der anderen. Der molekulare Diffusionskoeffizient lässt sich für ein solches binäres Gemisch nach Fuller [48] über die Gleichung 2.34 abschätzen.

$$
D_{1,2}=\frac{0,00143^{1,75}\left(\frac{1}{M_{1}}+\frac{1}{M_{2}}\right)^{1 / 2}}{p \cdot \sqrt{2}\left[\left(\sum \Delta_{V_{1}}\right)^{1 / 3}+\left(\sum \Delta_{V_{2}}\right)^{1 / 3}\right]^{2}}
$$

Soll der Stofftransport bei der Adsorption beschrieben werden, ist es prinzipiell notwendig, alle beteiligten Transportphänomene einzeln zu erfassen und zu beschreiben. Da dies aber nur unter hohem experimentellem und finanziellem Aufwand möglich ist, wurden vereinfachende Modelle entwickelt.

\section{Kinetisches Modell des Stofftransports (Linear-Driving-Force-Ansatz)}

Um die im Adsorbens ablaufenden Stofftransportmechanismen beschreiben zu können, gibt es verschiedene Modellvorstellungen [49]. Ein in der Praxis und auch der Forschung oft verwendeter Ansatz ist der kinetische LDF-Ansatz (Linear-Driving-Force) [49]. Bei diesem Modell wird gemäß der schematischen Darstellung in Abbildung 7 als Triebkraft für die zeitliche Änderung der Adsorbensbeladung eine Differenz zwischen der zur momentan gegebenen Fluidphasenkonzentration gehörenden Gleichgewichtsbeladung und einer mittleren, zu der jeweiligen Zeit vorliegenden Beladung angesetzt.

Bathen [47] gibt für diese Beziehung die Differentialgleichung

$$
\frac{\partial X}{\partial t}=k_{\text {eff }} \cdot \frac{A_{P}}{\rho_{P}} \cdot\left(X_{G l}-X\right)
$$

an. 


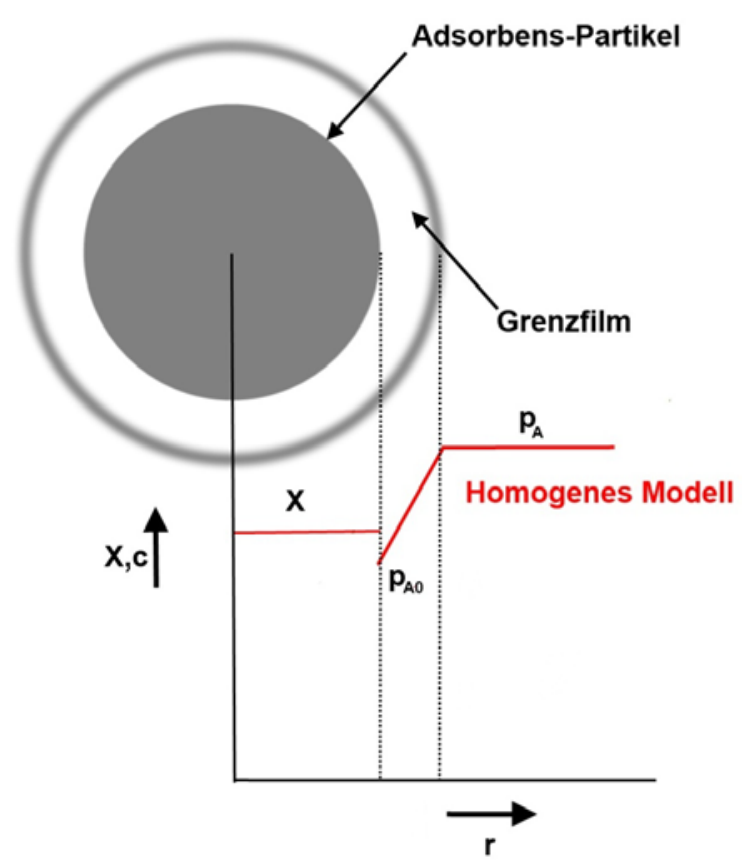

Abbildung 7: Schema Linear-Driving-Force-Ansatz

Der gesamte Stoffdurchgang, der sich in Grenzfilmdiffusion, Porendiffusion (Freie Porendiffusion + Knudsen Diffusion) und Adsorption unterteilt, wird in diesem Ansatz über einen globalen effektiven Stoffdurchgangskoeffizienten $k_{\text {eff }}$, einer spezifischen Oberfläche und die oben geschilderte Beladungsdifferenz beschrieben.

Mersmann [50] gibt zur Berechnung dieses effektiven Stoffdurchgangskoeffizienten unter Zuhilfenahme einer Abschätzung nach Glueckauf [51] die Formel

$$
k_{\text {eff }}=\frac{\rho_{P}}{A_{S p}} \cdot \frac{15}{r_{P}^{2}} \cdot \frac{D_{g e s} / \mu_{P, \text { Diff }}}{1+\frac{\rho_{P}}{\psi_{P}} \cdot \frac{\partial X}{\partial c_{A}}}
$$

an.

Für den effektiven Stoffdurchgangskoeffizienten bestehen nach dieser Gleichung neben adsorbensspezifischen Größen wie Partikelgröße, Adsorbensdichte- und porosität zwei veränderliche Abhängigkeiten: das Adsorptionsgleichgewicht und der Stofftransport. Auf Grund großer Relevanz für die spätere Diskussion kinetischer Effekte werden diese Abhängigkeiten weiter unten genauer ausgeführt.

Unter Annahme äquimolarer Diffusion lässt sich der in der Formel zusammengefasste Gesamtdiffusionskoeffizient als reziproke Überlagerung der dominanten Einzelmechanismen gemäß Gleichung 2.37 formulieren: 


$$
D_{\text {ges }}=\frac{1}{\left(\frac{1}{D_{K n}}+\frac{1}{D_{\text {Diff }}}\right)}
$$

Soll das zeitveränderliche Messsignal, die Durchbruchskurve, anhand dieses kinetischen Ansatzes beschrieben werden, müssen die einflussnehmenden Faktoren, die in erheblichem Maße die Form der Durchbruchskurve bestimmen, detailliert betrachtet werden.

\section{Einflussfaktoren auf die Form der Durchbruchskurve}

Mit Hilfe des eingeführten kinetischen LDF-Ansatzes lässt sich der gesamte Stofftransport der Adsorption durch einen globalen Parameter beschreiben. Dieser ist maßgebend für die Form der Durchbruchskurve und damit einhergehende Größen, wie z. B. die Breite der Massentransferzone. Anhand Gleichung 2.36 lassen sich zwei veränderliche Einflussfaktoren erkennen. Im Folgenden soll deren Einfluss auf die Prozessdynamik einer kontinuierlich durchströmten Schüttung betrachtet werden.

\section{Einfluss des Adsorptionsgleichgewichtes auf die Wanderungsgeschwindigkeit}

Nimmt man für eine Durchbruchskurve an, dass zwischen Adsorbens und Gasphase ständiges Gleichgewicht besteht und lässt weiter Geschwindigkeitsänderungen $\frac{\partial v}{\partial z}$ und axiale Dispersion $\mathrm{D}_{\mathrm{ax}}$ unberücksichtigt, bedeutet dies den Wegfall aller Stofftransportwiderstände. Für die Wanderungsgeschwindigkeit der Konzentrationsfront durch die Schüttung ergibt sich dann der Ausdruck [38]:

$$
u_{c_{A}}=\frac{u_{L R} / \psi}{1+\frac{1-\psi}{\psi} \cdot \rho_{S} \cdot \frac{\partial X}{\partial c_{A}}}
$$

Über die im Nenner enthaltene Isothermensteigung $\frac{\partial X}{\partial c}$ ist diese Geschwindigkeit vom Adsorptionsgleichgewicht abhängig.

Wird das Adsorptionsgleichgewicht z. B. durch eine Henry-Isotherme mit konstanter Steigung beschrieben, ist die Wanderungsgeschwindigkeit der Konzentrationsfront unabhängig von der Höhe der erreichten Beladung im Bett. Damit ist auch die Form der Durchbruchskurve 
unabhängig von der Beladung. Durchbruchskurven für verschiedene Gleichgewichtskonzentrationen haben in diesem Fall die gleiche Steigung und Form.

Wird im Gegensatz dazu das Gleichgewicht z. B. durch die Langmuir-Isotherme beschrieben, ergibt sich ein abweichendes Verhalten. Die Langmuir-Isotherme (vgl. Abschnitt 2.1.1) nähert sich mit steigender Konzentration des Adsorptivs in der fluiden Phase einem Beladungsgrenzwert. Somit ist die Steigung der Langmuir-Isotherme nicht konstant, sondern wird mit steigender Konzentration des Adsorptivs geringer, bis sie bei Erreichen des Beladungsgrenzwertes gegen Null geht. Entsprechend der o. g. Gleichung sollte die Wanderungsgeschwindigkeit für kleine Konzentrationen und Beladungen des Adsorptivs aufgrund der großen Steigung der Isotherme klein sein und zu höheren Konzentrationen und Beladungen hin ansteigen. Die neu und schwach beladenen, vorauslaufenden Bereiche der Massentransferzone wandern langsamer als die bereits stark beladenen, nachlaufenden Bereiche. Dies führt zu einer Aufsteilung der Durchbruchskurve mit zunehmender Wanderungsdauer. Bei einer hohen Gleichgewichtsbeladung ist dieser Effekt deutlicher ausgeprägt als bei einer geringen Gleichgewichtsbeladung. Abbildung 8 verdeutlicht diesen Effekt.

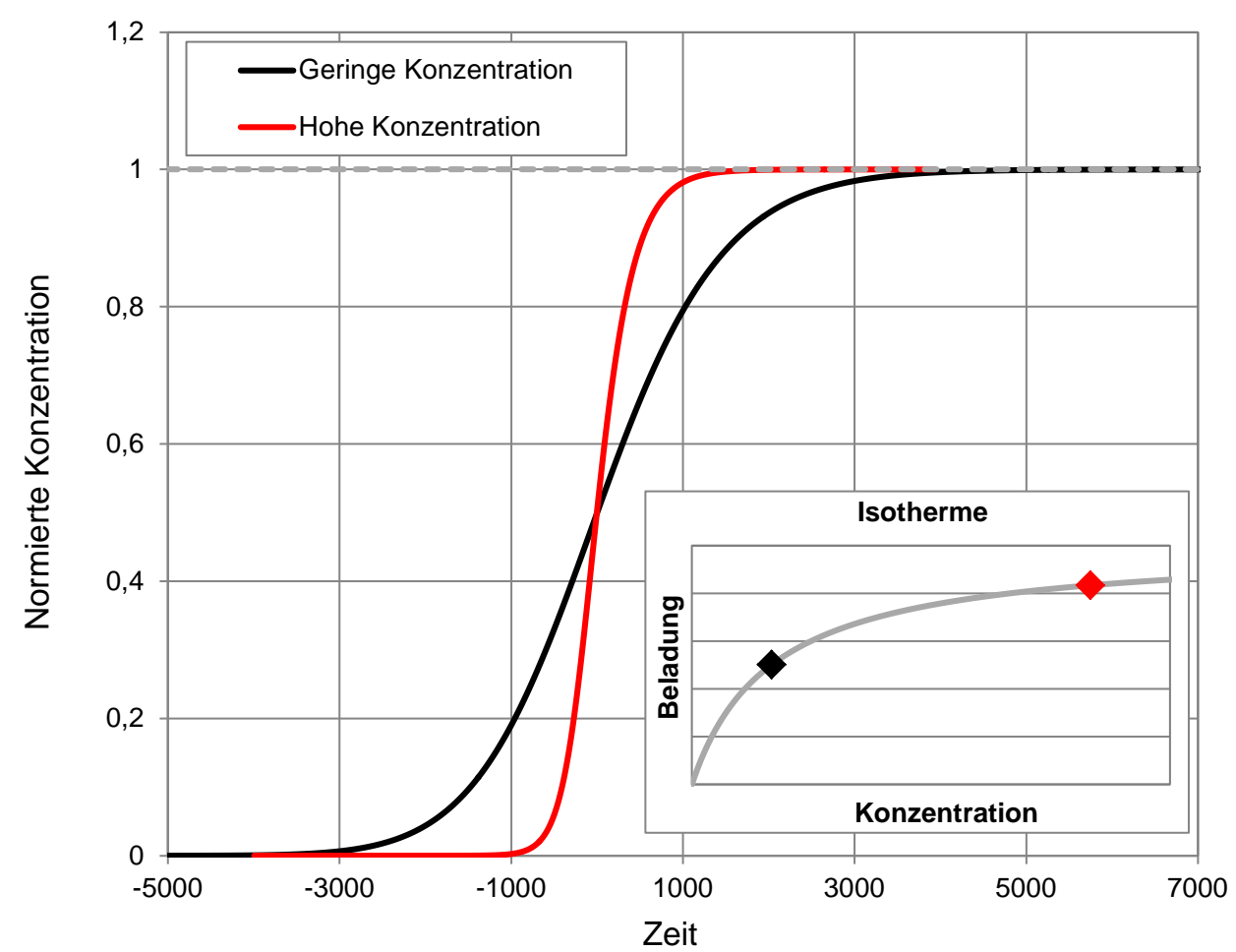

Abbildung 8: Durchbruchskurven bei unterschiedlichen Konzentrationen und konstanter Kinetik ( $\left.k_{\text {eff }}\right)$

Die Abbildung zeigt zwei normierte Durchbruchskurven bei unterschiedlicher Konzentration, gleichen Strömungsverhältnissen und gleichem $k_{\text {eff }}$ aufgetragen gegen die Zeit. Die Zeitachse beginnt nicht bei $\mathrm{t}=0$, sondern die Fronten sind zur Verdeutlichung der Darstellung mit 
ihren Schwerpunkten (Wendepunkt) auf den Zeitpunkt $\mathrm{t}=0$ gelegt. Es zeigt sich, dass die Durchbruchskurve bei geringer Konzentration gemäß Gleichung 2.38 deutlich flacher verläuft als diejenige bei höherer Konzentration. Die Verweilzeit der Front in der Schüttung ist größer bzw. die Massentransferzone ist breiter. Entsprechend ist die damit einhergehende Wanderungsgeschwindigkeit der Konzentrationsfront auch langsamer.

\section{Einfluss der Adsorptionskinetik}

Der Stofftransportwiderstand und das Adsorptionsgleichgewicht beeinflussen entsprechend des Linear-Driving-Force-Ansatzes den effektiven Stoffübergangskoeffizienten $\mathrm{k}_{\text {eff }}$ ( $\mathrm{vgl}$ Gleichung 2.36).

Im Vergleich zur Berechnung der Wanderungsgeschwindigkeit der Konzentrationsfront durch das Zwischenkornvolumen des Bettes wird zusätzlich neben dem Adsorptionsgleichgewicht auch die Diffusion berücksichtigt.

Um nun den Einfluss des Stofftransportwiderstandes auf die Form der Durchbruchskurve beschreiben zu können, müssen Durchbruchskurven bei unterschiedlicher Kinetik ( $\left.D_{\text {ges }}, k_{\text {eff }}\right)$ und gleichen Konzentrationen betrachtet werden. Abbildung 9 zeigt diese Betrachtungsweise für normierte Durchbruchskurven.

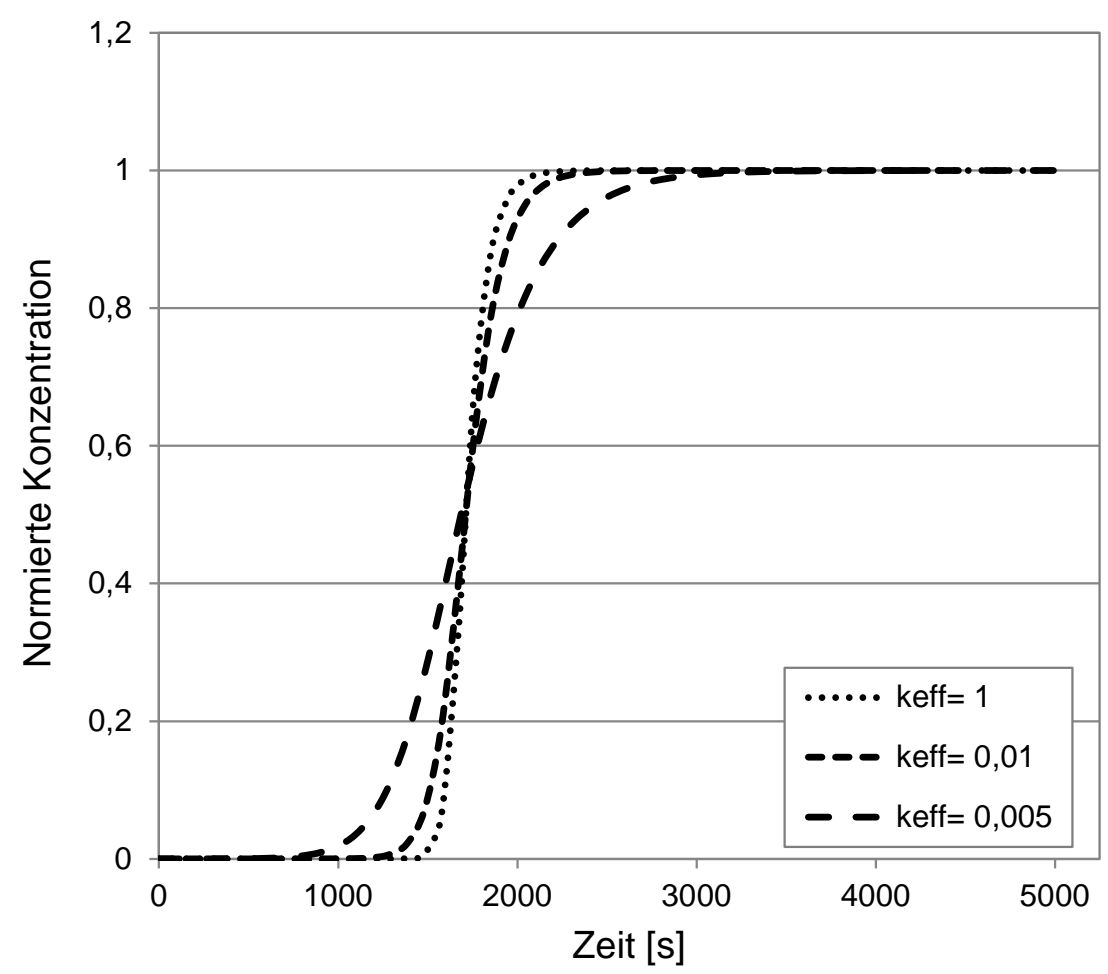

Abbildung 9: Durchbruchskurven bei gleichen Konzentrationen und veränderlicher Kinetik $\left(k_{\text {eff }}\right)$ 
Durch diese Betrachtung wird deutlich, dass die Kinetik und der damit einhergehende Stofftransportwiderstand einen ähnlichen Einfluss auf die Form der Durchbruchskurve haben wie das Adsorptionsgleichgewicht. Bei $k_{\mathrm{eff}}=1$ ist der Stofftransport ungehemmt. Zu kleineren

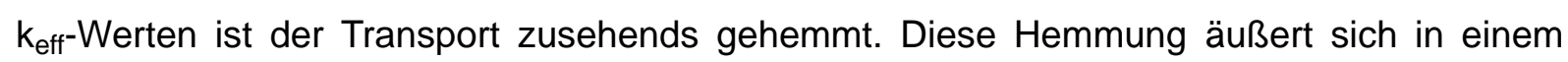
Abflachen der Front.

\section{Einfluss der axialen Dispersion}

Nimmt man an, dass eine Schüttung ohne jegliche Adsorptionseffekte durchströmt wird, zeigt sich dennoch eine Verbreiterung der Konzentrationsfronten, obwohl die Einflüsse des Adsorptionsgleichgewichtes und des Stofftransportes nicht bestehen. Dieser Effekt ist auf die axiale Dispersion zurückzuführen. Die Adsorbensschüttung bewirkt im Vergleich zum leer durchströmten Adsorber eine starke Querschnittsänderung. Infolgedessen entstehen große örtliche Geschwindigkeitsänderungen, die zu dieser Verbeiterung führen [38].

Unter Verwendung eines Ansatzes nach Wakao [52] gibt Bathen [47] für die axiale Dispersion Gleichung 2.39 an:

$$
D_{a x}=\frac{20}{\psi} \cdot D_{1,2}+0,5 \cdot \frac{u_{L R} \cdot d_{P}}{\psi}
$$

Im Vergleich zu den beschriebenen Einflussfaktoren auf die Durchbruchskurvenform ist der Einfluss durch die axiale Dispersion bei vernünftig gewählten Prozessparametern (Strömungsverhältnisse, Adsorbergeometrie, etc.) als gering einzustufen [38].

\subsection{Eigenschaften der eingesetzten Stoffe}

\subsubsection{Adsorbentien}

In der industriellen Praxis wird in der Erdgasaufbereitung aus dem Bereich der physisorptiv wirkenden Adsorbentien auf verschiedene Zeolithe und Silica-Gele zurückgegriffen. Wird die Aufbereitung durch Chemisorption erreicht, sind Aktivkohlen, die mit verschiedenen Chemikalien imprägniert sind, weit verbreitet. Im Folgenden soll auf Ausgangsmaterialien und Herstellung physisorptiv wirkender Adsorbentien eingegangen werden, da die experimentellen Untersuchungen im Rahmen dieser Arbeit mit solchen Materialien durchgeführt wurden. 
Für eine detaillierte Betrachtung der Herstellungsverfahren und Wirkweisen modifizierter oder imprägnierter Aktivkohlen sei an dieser Stelle auf die relevante Literatur verwiesen [53].

\section{Silica-Gel (Kieselgel)}

Silica-Gel gehört zur Gruppe der oxidischen Adsorbentien. Es besteht zum Großteil aus amorphem Siliziumdioxid. Die Herstellung erfolgt in einem Sol-Gel-Verfahren aus Alkalisilikatlösung. Wird die Lösung neutralisiert oder angesäuert, bildet sich Kieselsäure $(\mathrm{Si}(\mathrm{OH}) 4)$, welche in einer Polykondensation zu Partikeln aggregiert. Entscheidend für diesen Prozess ist der pH-Wert [54]. Es folgt ein Waschschritt, bei dem Kationen ausgetauscht werden. In einem darauffolgenden Trocknungsschritt koagulieren die Partikel und bilden eine poröse Struktur aus, das Silica-Gel. Ein charakteristisches Merkmal von Silica-Gel sind die oberflächlichen $\mathrm{OH}$-Gruppen und die daraus folgende Polarität der Oberfläche. Aus diesem Grund lassen sich polare Moleküle wie z. B. Wasser unter Bildung von Wasserstoffbrücken gut adsorbieren.

\section{Zeolithe (Molekularsiebe)}

Bei Zeolithen handelt es sich um natürlich vorkommende oder synthetisch hergestellte Alumosilikate, die zusätzlich Alkali- oder Erdalkalikationen enthalten. Die chemische Zusammensetzung dieser hochporösen, kristallinen Feststoffe mit genau definierter Porenstruktur lässt sich über die Summenformel

$$
M_{x / n}\left[\left(\mathrm{AlO}_{2}\right)_{x}\left(\mathrm{SiO}_{2}\right)_{y}\right] \cdot \mathrm{ZH}_{2} \mathrm{O}
$$

beschreiben [35]. Hierbei beschreibt $n$ die Valenz des Kations $M . X$ und $Y$ sind ganzzahlig und das Verhältnis y/x nimmt einen Wert größer gleich 1 an. Die primäre Grundstruktur aller Zeolithe bilden Tetraeder der Elemente Silizium und Aluminium. Diese Grundelemente lassen sich zu verschiedenen geometrischen Anordnungen, den so genannten Secondary Building Units (SBUs), zusammenfügen. Durch Kombination verschiedener SBUs lassen sich beliebige Gitterstrukturen herstellen. Dies sei im Folgenden anhand des Beispiels in Abbildung 10 erläutert. Einer der bekanntesten tertiären Strukturen ist der Sodalith-Käfig (c). Er besteht aus 24 Tetraedern bzw. acht Sechsereinfach-SBUs (a). Verknüpft man acht Sodalith-Käfige über Viererdoppelringe (b) miteinander, führt dies zu einem elementaren LTAMolekularsiebgitter (Zeolith A, d). 


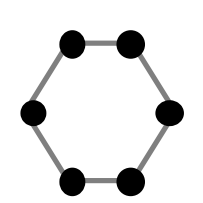

a

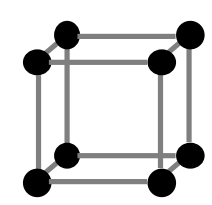

$\mathrm{b}$

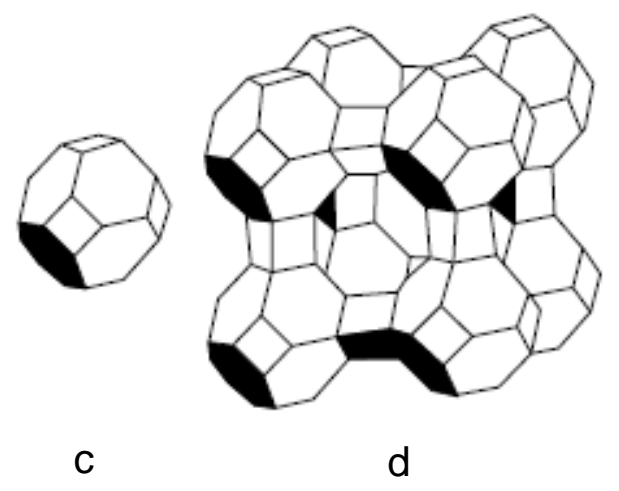

Abbildung 10: a, b: Secondary Building Units: Sechsereinfachring und Viererdoppelring, c: SodalithKäfig, d: Elementarzelle des Typ A Zeolith [35]

Der freie Durchmesser des sich ergebenden zentralen Käfigs, auch Alpha-Käfig genannt, besitzt eine Größe von 11,4 A. Adsorptive können durch sechs Sauerstoff-Achtecke mit einer jeweiligen Größe von 4,4 $\AA$ in diesen Käfig diffundieren. In jeder Elementarzelle werden 12 negative Ladungen der Aluminiumtetraeder durch Kationen ausgeglichen. Typ A Zeolithe werden als Ausgangsform mit Natriumkationen synthetisiert. Durch Austausch der Natriumkationen mit anderen Metallen lassen sich gezielt andere Porengrößen einstellen. Beispielsweise wird ein 3A-Zeolith durch den Austausch mit Kaliumkationen hergestellt; ein 5A-Zeolith mit Austausch durch Kalzium.

Je nach Silizium/Aluminium-Verhältnis bilden sich verschiedene Grundgerüste und dadurch auch verschiedene Gitterstrukturen aus, wie z. B. Typ Y- oder X-Zeolithe.

Die Herstellung von Zeolithen erfolgt über Kristallisation aus basischen Lösungen von Wasserglas, welches die Silikat-Komponente darstellt, und Aluminiumhydroxid bei definierter Temperatur (bis ca. $200^{\circ} \mathrm{C}$ ) [47]. Anschließend folgen ein Abfiltern der gebildeten Kristalle und ein Trocknungsschritt. Zuletzt werden Ionen ausgetauscht und der Binder zugefügt, um sphärische Partikel zu erhalten. Der Markt bietet jedoch je nach Applikation auch binderfreie Zeolithe an. Diese Zeolithe stellen dann ausschließlich Mikroporenvolumen zur Verfügung. Der mesoporöse Porenanteil des Binders entfällt. 


\subsubsection{Trägergase und Adsorptive}

Der Schwerpunkt der im Rahmen dieser Arbeit untersuchten Adsorptive liegt auf Molekülen, die das Atom Schwefel beinhalten. Da es in einem realen Erdgasaufbereitungsprozess jedoch immer zu einer simultanen Adsorption mehrerer Verbindungen kommen wird, wurden in diesem Zusammenhang ebenfalls Untersuchungen zu den Adsorptiven Wasser und Kohlendioxid durchgeführt. Dazu wurden als Grundlage Adsorptionsisothermen dieser beiden Verbindungen aus binären Gasmischungen (Trägergas $+\mathrm{H}_{2} \mathrm{O}$ oder $\mathrm{CO}_{2}$ ) vermessen (Abschnitt 4.3), um in der Folge Effekte wie Koadsorption oder Verdrängung bei der Untersuchung ternärer Stoffsysteme (Trägergas $+\mathrm{S}$-Verbindung $+\mathrm{H}_{2} \mathrm{O}$ oder $\left.\mathrm{CO}_{2}\right)($ Abschnitt 4.4) beschreiben zu können.

In allen Untersuchungen wurde die Adsorption aus einem Trägergas betrachtet. Im Vergleich zu Reinstoffisothermen, die üblicherweise volumetrisch oder gravimetrisch in statischen Versuchsanordnungen vermessen werden, bietet die Messung von Adsorptionskapazitäten, die in dynamischen Anordnungen mit Hilfe kontinuierlich durchströmter Apparate ermittelt werden, die Möglichkeit, industrielle Aufbereitungsprozesse realistischer beschreiben und Adsorptionskapazitäten applikationsspezifisch genauer ermitteln zu können. Die Nachteile dieser Methode sind die hohen Betriebskosten auf Grund des Materialeinsatzes und der zeitliche Aufwand.

Dabei kann unter Umständen die Wahl des Trägergases von großer Bedeutung sein. Wird beispielsweise die Spurenabtrennung untersucht, liegt das verwendete Trägergas in sehr hoher Konzentration vor (> 99 \%). Die Koadsorption des Trägergases ist in diesem Falle nicht zu vernachlässigen und kann in der Größenordnung der Adsorptionskapazitäten der eigentlichen Zielkomponente liegen. Entsprechend ist mit einer starken Beeinflussung des Adsorptionsprozesses und deutlich unterschiedlichen Ergebnissen für die Adsorption einer Schwefelverbindung aus Erdgas im Vergleich zur Reinstoffadsorption zu rechnen. Für die hier verwendeten Trägergase Methan und Stickstoff konnten beispielsweise im Falle der Adsorption 5A Zeolith deutliche Unterschiede nachgewiesen werden (vgl. Abschnitt 4.2.3). Diese lassen sich anhand der Reinstoffisothermen der betreffenden Gase verstehen [20].

\section{Trägergase}

Stickstoff $\left(\mathrm{N}_{2}\right)$ ist ein farb- und geruchloses nicht brennbares Gas. Auf Grund seiner Dreifachbindung gilt Stickstoff als äußerst reaktionsträge und wird daher häufig als Inertgas (z. B. Schweißschutzgas oder Reifengas) eingesetzt. Die Atmosphäre der Erde besteht zu ca. 78\% 
aus Stickstoff. Reiner Stickstoff wird heutzutage in Luftzerlegungs- oder mehrstufigen Adsorptions-/Desorptionsanlagen gewonnen. In der Adsorptionstechnik wird Stickstoff zur physikalischen Charakterisierung von Adsorbentien bei $77 \mathrm{~K}$ verwendet.

Methan $\left(\mathrm{CH}_{4}\right)$ ist ein farbloses, brennbares Gas. Es ist das einfachste Molekül der Gruppe der Alkane. Das Molekül hat eine tetraedrische Geometrie. Es weist keinen Dipol auf und bildet keine Wasserstoffbrückenbindungen aus. Sowohl in Erdgas als auch in regenerativ hergestellten Gasen ist Methan Hauptbestandteil. Hauptverwendungen sind der Gebrauch als Brennstoff und als Ausgangsstoff in der chemischen Industrie für zahlreiche Synthesen wie z. B. für Methanol [55].

\section{Adsorptive}

Wasser $\left(\mathrm{H}_{2} \mathrm{O}\right)$ ist ein gewinkeltes Molekül aus den Atomen Sauerstoff und Wasserstoff. Die unterschiedlichen Elektronegativitäten der Atome sorgen für Partialladungen innerhalb des Wassermoleküls. Das Sauerstoffatom trägt im Wassermolekül eine negative Partialladung, die beiden Wasserstoffe eine positive Partialladung. Auf Grund der gewinkelten Geometrie und der deutlich unterschiedlichen Partialladungen besitzt Wasser ein Dipolmoment, wodurch es in der Lage ist, Wasserstoffbrückenbindungen einzugehen. Dadurch zeigt Wasser eine hohe Affinität zu polaren Adsorbentien wie Zeolithen und Silica-Gelen.

Kohlendioxid $\left(\mathrm{CO}_{2}\right)$ ist ein farbloses, geruchloses, nicht brennbares Gas. Die Molekülgeometrie ist linear. Die beiden Sauerstoffatome sind über Doppelbindungen an den Kohlenstoff gebunden. Auf Grund der symmetrischen Struktur des Moleküls besitzt es kein elektrisches Dipolmoment, wohl aber ein Quadrupolmoment. Bei der Verbrennung von kohlenwasserstoffhaltigen Verbindungen mit Sauerstoff entsteht es als Reaktionsprodukt. In Erdgasen kann der Kohlendioxidgehalt bis in den Prozentbereich reichen.

Schwefelwasserstoff $\left(\mathrm{H}_{2} \mathrm{~S}\right)$ ist ein toxisches, unangenehm riechendes korrosives Gas. Der Molekülaufbau des $\mathrm{H}_{2} \mathrm{~S}$ ist ähnlich wie bei $\mathrm{H}_{2} \mathrm{O}$ gewinkelt. Durch die unterschiedlichen Elektronegativitäten des Schwefelatoms und der Wasserstoffatome besitzt das Molekül auf Seiten des S-Atoms eine negative- und auf Seiten der H-Atome positive Partialladungen. Daraus resultiert ein elektrisches Dipolmoment. $\mathrm{H}_{2} \mathrm{~S}$ entsteht aus Zersetzungsprozessen organischer Verbindungen. Geringe Mengen in unmittelbarer Umgebung führen zu einer Reizwirkung der Augen und des Rachenraums. Der Schwellenwert für eine längere Exposition liegt bei 10 $\mathrm{ppm}_{\mathrm{v}}$. Konzentrationen ab einem Wert von 0,1\% wirken innerhalb weniger Minuten tödlich. Die eigentliche Gefahr besteht in einer Betäubung des Geruchsinns ab einer Schwefelwasserstoff-Konzentration $>250$ ppm $_{\vee}$ [56]. Dadurch sind exponierte Personen nicht mehr in der 
Lage, Schwefelwasserstoff wahr zu nehmen. In Verbindung mit hohen Feuchtigkeitsgehalten bildet $\mathrm{H}_{2} \mathrm{~S}$ saure, korrosive Lösungen. Weiterhin kann es in Anwesenheit von $\mathrm{H}_{2} \mathrm{~S}$ zu Spannungsrisskorrosion bei bestimmten Stahlwerkstoffen kommen [57]. Bei der Verbrennung bildet sich ebenfalls korrosives Schwefeldioxid, welches in der Atmosphäre in Verbindung mit Wasser und Sauerstoff zu saurem Regen führen kann. Derartige atmosphärische Schwefelemissionen sind gesetzlich strikt begrenzt [58].

Liegen Schwefelwasserstoff und Kohlendioxid in höheren Konzentrationen im Erdgas vor, sind meistens auch weitere Schwefelverbindungen wie Mercaptane oder Carbonylsulfid vorhanden [3].

Mercaptane (z. B. $\mathrm{CH}_{3} \mathrm{SH}, \mathrm{C}_{2} \mathrm{H}_{5} \mathrm{SH}, \mathrm{C}_{3} \mathrm{H}_{7} \mathrm{SH}$ ), auch Thioalkohole genannt, sind Kohlenwasserstoffe mit aliphatisch gebundener, funktioneller Thiolgruppe (-SH). Sie entsprechen formal den Alkoholen, die anstatt der funktionellen Thiolgruppe eine Hydroxygruppe (-OH) tragen. Alkohole lassen sich formal vom Wasser ableiten; Mercaptane von $\mathrm{H}_{2} \mathrm{~S}$. Die geringere Elektronegativität des Schwefelatoms im Vergleich zu Sauerstoff führt zu einer geringeren Assoziation und zu einer höheren Flüchtigkeit der Thioalkohole [59]. Die im Rahmen dieser Arbeit betrachteten kurzen Homologe der Thioalkohole besitzen ähnliche Eigenschaften wie Schwefelwasserstoff. Methylmercaptan ist ein unangenehm riechendes Gas, welches bei der Odorierung von Erd- oder Propangas Verwendung findet. Ethyl- und Propylmercaptan sind unter Umgebungsbedingungen sehr stark riechende Flüssigkeiten. Ethylmercaptan wird unter anderem als Odoriermittel für Flüssiggas eingesetzt. Alle drei betrachteten Mercaptane sind Ausgangsstoffe für zahlreiche Synthesen in der chemischen Industrie. Methylmercaptan findet z. B. Verwendung bei der Herstellung der Aminosäure Methionin, welche als Nahrungsergänzung für Futtermittel benötigt wird [60].

Carbonylsulfid (COS) ist ebenso wie Schwefelwasserstoff oder Methylmercaptan ein toxisches, brennbares, unangenehm riechendes Gas, welches in sehr geringen Konzentrationen in Erdgasen enthalten ist. Die Molekülstruktur ist linear. Sauerstoff und Schwefel sind jeweils über Doppelbindungen mit dem Kohlenstoff verbunden. Auf Grund der unterschiedlichen Elektronegativitäten von Sauerstoff und Schwefel besitzt COS ein geringes elektrisches Dipolmoment. Unter bestimmten Voraussetzungen kann es in adsorptiven Erdgasaufbereitungsprozessen in Anwesenheit von Schwefelwasserstoff und Kohlendioxid zur COS-Bildung gemäß der Reaktionsgleichung

$$
\mathrm{H}_{2} \mathrm{~S}+\mathrm{CO}_{2} \rightleftharpoons \mathrm{COS}+\mathrm{H}_{2} \mathrm{O}
$$

kommen [61]. Diese Thematik wird in Abschnitt 4.4 noch genauer diskutiert. 


\subsection{Wechselwirkungen und Polarität}

Adsorbieren Moleküle physikalisch, findet dies auf Grund von Anziehungskräften statt. Grundsätzlich spielt dabei die Ladungsverteilung in den Adsorptivmolekülen und auf der Adsorbensoberfläche eine wichtige Rolle.

Zu den Anziehungskräften zählen in jedem Fall van der Waals-Kräfte oder Dispersionskräfte. Bei polaren Adsorbentien oder Adsorptiven kommt es darüber hinaus zu elektrostatischen Wechselwirkungen. Hierzu zählen permanente Dipol-Dipol-Wechselwirkungen, induzierte Dipol-Wechselwirkungen, Wasserstoffbrückenbindungen, Ion-Dipol-Wechselwirkungen und koordinative Wechselwirkungen. Zum Beispiel sind für die Bindung eines Moleküls an einer ionischen Zeolith-Oberfläche Ion-Dipol-Wechselwirkungen von großer Bedeutung, für eine Wechselwirkung eines Moleküls mit einem Silica-Gel vor allem Wasserstoffbrückenbindungen mit den oberflächlichen $\mathrm{OH}-G r u p p e n$.

Die Polarität eines Moleküls entsteht durch die unterschiedlichen Elektronegativitäten der Atome im Molekül. Hat ein Atom innerhalb einer Bindung die Fähigkeit, Elektronen stärker an sich zu ziehen als ein anderes Atom, entsteht eine Ladungsverschiebung, die zu einer positiven Partialladung auf dem schwächer anziehenden und einer negativen Partialladung auf dem stärker anziehenden Atom führt. Damit bildet sich eine polare Bindung oder ein lokales Dipolmoment aus. In einem Molekül mit mehreren polaren Bindungen addieren sich die lokalen Dipolmomente zum molekularen Dipolmoment. Das molekulare Dipolmoment kann indirekt z. B. mit Hilfe der Debye-Gleichung über die Messung der Permitivität bestimmt werden [62].

Ein Nachteil der Verwendung des Dipolmoments zur Beschreibung von AdsorbensAdsorptiv-Wechselwirkungen ist, dass es lediglich die Gesamtpolarität eines Moleküls beschreibt. Für die Adsorption kann man sich aber Wechselwirkungen zwischen lokalen Dipolen des Adsorptivs und der Oberfläche vorstellen, so dass das molekulare Dipolmoment des Adsorptivs allein als Kriterium zur Beurteilung der Wechselwirkungen nicht ausreicht. Neben dem molekularen Dipolmoment ergeben sich aus Abweichungen der Ladungsverteilung von der Kugelgeometrie auch höhere Momente wie das molekulare Quadrupolmoment, die bei bestimmten Molekülgeometrien ebenfalls eine Rolle spielen können. So besitzt beispielsweise das linear symmetrisch aufgebaute $\mathrm{CO}_{2}$-Molekül trotz seiner stark polaren Bindungen kein Dipolmoment, sondern ein Quadrupolmoment.

Die Coulomb-, Dipol- und Quadrupolwechselwirkungen stellen die ersten und damit wichtigsten Beiträge der sogenannten Multipolentwicklung dar. Einen vertiefenden Einblick in diese 
Thematik gewährt Lucas [63]. Am Beispiel des Moleküls $\mathrm{CO}_{2}$ lässt sich anhand der experimentellen Ergebnisse dieser Arbeit zeigen, dass die Stärke der QuadrupolDipolwechselwirkung im Vergleich zu einer Dipol-Dipolwechselwirkung als geringer einzuschätzen ist. Dennoch sollte sie nicht vernachlässigt werden.

\subsection{Quantenmechanische Berechnung von Partialladungen}

Um die lokale Ladungsverteilung besser veranschaulichen zu können, wurden quantenmechanische Methoden zur Berechnung von Partialladungen verwendet. Hierunter gibt es zahlreiche Berechnungsverfahren. Es kann zwischen ab initio Verfahren (z. B. Hartree-Fock Verfahren) und semiempirischen Verfahren (Dichte-Funktional-Theorie Methoden (DFT)) unterschieden werden. Beide Verfahren basieren auf der Lösung der Schrödinger-Gleichung. Im Rahmen dieser Arbeit wurden Partialladungen von Atomen unter Verwendung der DichteFunktional-Theorie berechnet. Die Dichtefunktionaltheorie basiert auf der Strategie, die Elektronenkorrelation (Dynamische Wechselwirkungen jedes einzelnen Elektrons mit jedem einzelnen anderen Elektron) mit Funktionalen der Elektronendichte zu beschreiben. Es wird versucht die Elektronendichte im energetischen Grundzustand eines Moleküls zu bestimmen. Grundlage der Theorie ist das Hohenberg-Kohn-Theorem. Zur Vertiefung sei an dieser Stelle auf die relevante Literatur verwiesen [63].

Die Partialladungen wurden aus dem molekularen, elektrostatischen Potenzial (Merz-SinghKollman-Methode) berechnet.

Für Moleküle, die das Atom Schwefel enthalten, ist die Bestimmung der Partialladungen mit Hilfe dieser Methode sinnvoll [65]. Alle Berechnungen dieser Arbeit wurden mit dem Programm GAUSSIAN 03 durchgeführt. Tabelle 2 gibt eine Auflistung der gewählten Methode und Parameter der Partialladungsberechnung.

Tabelle 2: Berechnungsmethodik und Parameter für die Partialladungen

\begin{tabular}{|l|l|}
\hline Berechnungsprogramm & GAUSSIAN 03 \\
\hline Berechnungsjob & Minimierung (Energie/Geometrie) \\
\hline Methode & Dichtefunktionaltheorie \\
\hline Funktional & B3LYP \\
\hline Basissatz & $6-311+G{ }^{\star *}$ \\
\hline Schalenmodell & geschlossen \\
\hline Berechnung der Partialladungen nach & $\begin{array}{l}\text { Molecular Electrostatic Potential } \\
\text { (Merz-Singh-Kollman) }\end{array}$ \\
\hline
\end{tabular}




\subsection{Physikalisches Modell der Gasphasenadsorption}

Zur mathematischen Beschreibung der Festbettvorgänge ist es notwendig, die ablaufenden Stoff- und Wärmetransportmechanismen formelmäßig zu erfassen. Da es sich in diesem Falle um ein instationäres Problem handelt, erhält man vier gekoppelte Partielle Differentialgleichungen (2 Massenbilanzen, 2 Energiebilanzen). Im Rahmen dieser Arbeit wurde die Adsorption im Spurenbereich untersucht. Die während des Adsorptionsprozesses messbare Wärmetönung in Schüttung und Gas ist derart gering, dass für die Modellierung vereinfachend eine isotherme Adsorption angenommen wurde. Dementsprechend verbleiben für die mathematische Beschreibung der Adsorption nur die Massenbilanzen für fluide Phase und feste Phase. Um diese Bilanzen nummerisch lösen zu können, bedarf es eines mathematischen Bilanzraumes. Dazu wird der Adsorber gemäß dem in Abbildung 11 dargestellten Schema diskretisiert.
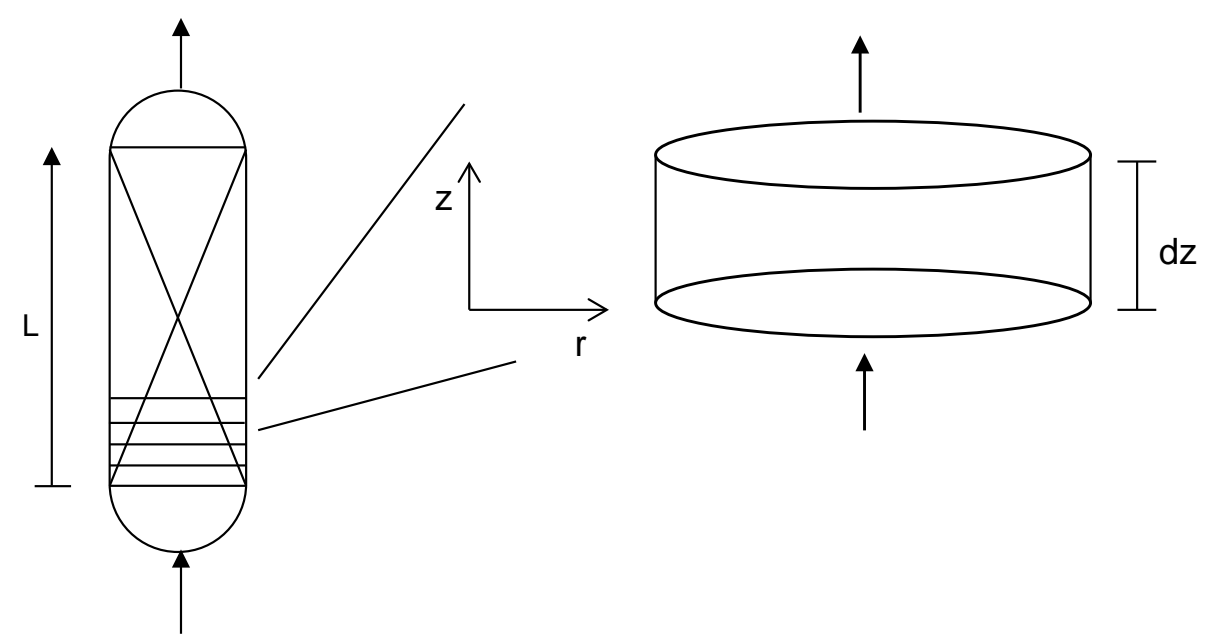

Abbildung 11: Bilanzraum für die Modellierung der Adsorption

Der Adsorber wird in $\mathrm{n}$ finite Volumenelemente der Höhe $\mathrm{dz}$ unterteilt. Jedes dieser Volumenelemente enthält unter Annahme einer homogenen Schüttung die gleiche Anzahl an Adsorbenspartikeln.

Folgende weitere vereinfachende Annahmen liegen der Massenbilanzierung zugrunde [47]:

1. Das Modell bildet die Adsorption einer Komponente bei isothermen Bedingungen ab Die Energiebilanzen für die feste und fluide Phase werden somit vernachlässigt. Dies ist bei der Untersuchung der Adsorption im Spurenbereich gerechtfertigt, wenn die Wärmetönung gering ist. Eine Adsorption des Trägergases findet nicht statt. Diese Annahme rechtfertigt sich auf Grund der experimentellen Prozedur (vgl. Abschnitt 3.2.3). 
2. Die fluide Phase unterliegt dem idealen Gasgesetz.

3. Es besteht ein konstanter Strömungsquerschnitt, d.h. es herrschen eine konstante Bettporosität und Adsorbenseigenschaften.

4. Radiale Strömungs- oder Konzentrationsgradienten werden vernachlässigt.

5. Die Geometrie des Bilanzraumes wird als konstant angenommen. Einflüsse durch Störungen der Betthomogenität (Temperatursensoren) werden vernachlässigt.

6. Konzentrationsgradienten im Adsorbenspartikel werden vernachlässigt. Beschreibung des Stofftransportwiderstandes über einen effektiven Stofftransportkoeffizienten (Linear-Driving-Force-Ansatz).

7. Der Prozess findet bei konstantem Druck statt.

8. Ein Druckverlust über der Schüttung wird vernachlässigt.

9. Ein kinetischer Einfluss durch axiale Dispersion der fluiden Phase wird berücksichtigt.

Für jedes numerische Diskret (Volumenelement der Höhe z) werden die Bilanzgleichungen gelöst. Im Folgenden werden die Bilanzgleichungen für fluide und feste Phase aufgestellt. Hierbei orientiert sich die Arbeit an der Herleitung von Bathen [47].

\section{Massenbilanz der festen Phase}

Für die Massenbilanz der festen Phase verhält sich die zeitlich im Adsorbens akkumulierte Masse gemäß Abbildung 12 zum adsorbierenden Massenstrom.

$$
\frac{\partial m_{S p}}{\partial t}=d \dot{m}_{A d s}
$$

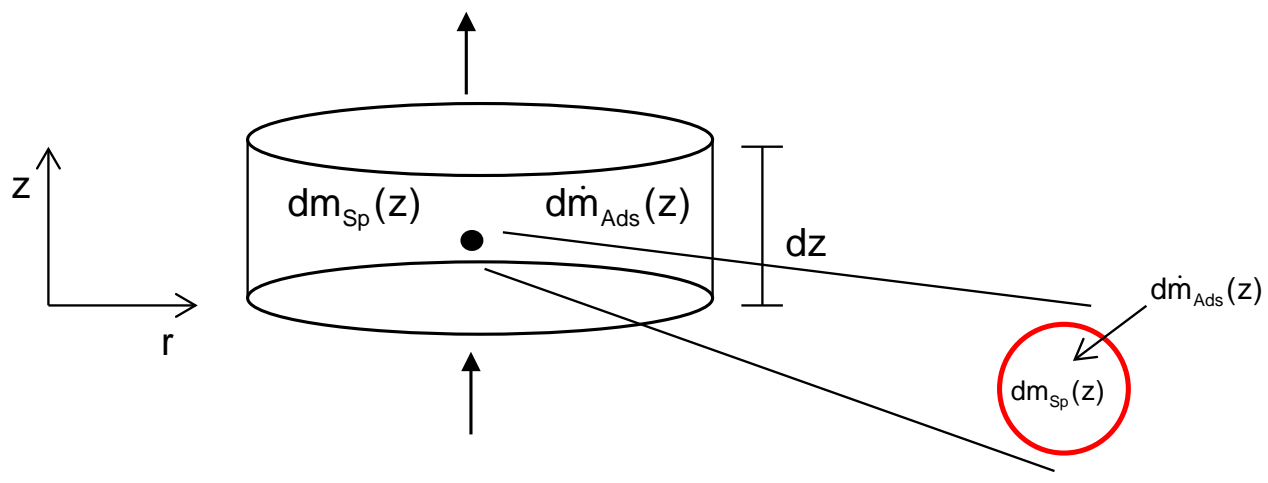

Abbildung 12: Schema zur Herleitung der Massenbilanz der festen Phase

Bezieht man den adsorbierenden Massenstrom auf die Adsorbensmasse pro Diskret, drückt also beide Terme durch die Adsorbensbeladung aus, und wendet den LDF-Ansatz mit der 
Differenz zwischen der realen gemittelten Adsorbensbeladung und der Gleichgewichtsbeladung als Triebkraft an, ergibt sich aus Gleichung 2.42 für die instationäre Massenbilanz der festen Phase die Differentialgleichung:

$$
\frac{\partial X}{\partial t}=\frac{k_{\text {eff }} \cdot A_{S p}}{\rho_{S}} \cdot\left(X_{G l}-X\right)
$$

\section{Massenbilanz der fluiden Phase}

Für die Massenbilanz der fluiden Phase sind örtliche Konzentrationsänderungen und Volumenstromänderungen infolge des Stofftransports bedingt durch axiale Dispersion und Konvektion zu berücksichtigen. Analog zur Massenbilanz der festen Phase ergibt sich für den aus dem Gasstrom adsorbierenden Stoffstrom ein Austauschterm mit entgegengesetztem Vorzeichen. Abbildung 13 zeigt die resultierenden Terme im Schema.

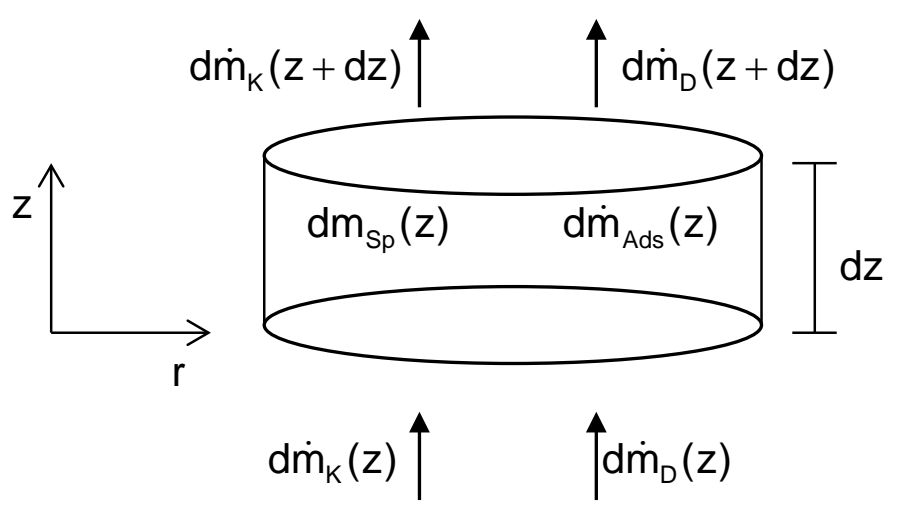

Abbildung 13: Schema zur Herleitung der Massenbilanz der fluiden Phase

Berücksichtigt man alle Terme ergibt sich Gleichung 2.44:

$$
\frac{\partial m_{S p}}{\partial t}=\dot{m}_{K}(z)-\dot{m}_{K}(z+d z)+\dot{m}_{D}(z)-\dot{m}_{D}(z+d z)-d \dot{m}_{A d s}
$$

Durch Umrechnung in Konzentration und Volumen der fluiden Phase im Diskret ergibt sich mit der Adsorberquerschnittsfläche A die partielle Differentialgleichung:

$$
\frac{\partial c_{A}}{\partial t}=D_{a x} \frac{\partial^{2} c_{A}}{\partial z^{2}}-\frac{\dot{V}_{G}}{A \cdot \psi} \frac{\partial c_{A}}{\partial z}-\frac{c_{A}}{A \cdot \psi} \frac{\partial \dot{V}_{G}}{\partial z}-\frac{k_{e f f} A_{S p}(1-\psi)}{\psi}\left(X_{G l}-X\right)
$$

Nach Kast [38] darf für geringe Adsorptiv-Konzentrationen eine Veränderung des Gesamtvolumenstroms vernachlässigt werden. Durch diese Annahme vereinfacht sich Gleichung 2.45 zu: 


$$
\frac{\partial c_{A}}{\partial t}=D_{a x} \frac{\partial^{2} c_{A}}{\partial z^{2}}-\frac{\dot{V}_{G}}{A \cdot \psi} \frac{\partial c_{A}}{\partial z}-\frac{k_{e f f} A_{S p}(1-\psi)}{\psi}\left(X_{G l}-X\right)
$$

\section{Anfangs - und Randbedingungen}

Die oben geschilderte Beschreibung der instationären Adsorption stellt ein Anfangswertproblem dar, welches numerisch nicht ohne die Wahl geeigneter Anfangs- und Randbedingungen gelöst werden kann. Folgende Anfangs- und Randbedingungen wurden zur Lösung des Gleichungssystems definiert:

Zum Zeitpunkt $\mathrm{t}=0$ herrscht in der Schüttung thermodynamisches Gleichgewicht. Für unbeladenes Adsorbens wurde

$$
\begin{aligned}
& c_{i}(z, t=0)=0 \\
& X_{i}(z, t=0)=0
\end{aligned}
$$

angenommen.

Für den eintretenden Gasstrom müssen für den Fall der isothermen Adsorption Gasvolumenstrom und Adsorptivkonzentration definiert werden

$$
\begin{aligned}
& \dot{V}_{G}(z=0, t)=\dot{V}_{G, 0} \\
& c_{i}(z=0, t)=c_{0}
\end{aligned}
$$

Durch diese Randbedingungen wird das Adsorptiv sprunghaft mit der definierten Konzentrationen auf die anfänglich unbeladene Schüttung aufgegeben.

Auf Grund der Verwendung eines halboffenen Modells wird die axiale Dispersion am Adsorberaustritt zu Null gesetzt [47].

$$
\frac{\partial^{2} C_{A}}{\partial z^{2}}(z=L, t)=0
$$

\section{Numerische Lösung der Modellgleichungen}

Zur numerischen Lösung der gekoppelten partiellen Differentialgleichungen sowie der Hilfsgleichungen wurde die Software Aspen Custom Modeler verwendet. Die Partiellen 
Differentialgleichungen werden in gewöhnliche Differentialgleichungen überführt. Durch örtliche und zeitliche Diskretisierung werden diese auf Grundlage der Finite-DifferenzenMethode nach der Euler-Methode [66] numerisch gelöst. 


\section{$3 \quad$ Experimentelles}

\section{1 Übersicht}

Im folgenden Abschnitt werden die verwendeten Adsorbentien, Adsorptive und Trägergase vorgestellt. Um die experimentelle Vorgehensweise für die im Rahmen der Arbeit durchgeführten Untersuchungen zu erläutern, werden die Versuchsanlage sowie die Durchführung der Messungen beschrieben. Es folgt eine Erläuterung der Methoden der Versuchsauswertung und der Approximation der Messdaten. Die Diskussion der Messfehler und der Reproduzierbarkeit der Versuche bilden den Abschluss des Abschnitts.

\subsubsection{Adsorbentien}

Sämtliche im Rahmen dieser Arbeit durchgeführten Adsorptionsuntersuchungen wurden an zwei im Bereich der Erdgasaufbereitung eingesetzten Adsorbentien durchgeführt. Es wurden ein mikroporöser Zeolith des Typs 5A und ein mesoporöses Silica-Alumina-Gel verwendet. Die Adsorbentien wurden von der BASF Catalysts GmbH Germany zur Verfügung gestellt. Darüber hinaus wurden zu Vergleichszwecken ergänzende Untersuchungen an zwei vom Hersteller modifizierten Silica-Alumina-Gelen durchgeführt. Zur Bestimmung struktureller Charakteristika wurden für alle Adsorbentien Stickstoffisothermen bei $77 \mathrm{~K}$ mit einer volumetrischen Sorptionsapparatur Belsorp-Max von Bel Japan, Inc. gemessen. Die Adsorbentien wurden vor den Sorptionsmessungen nach Herstellerangaben unter Vakuum $\left(<10^{-3} \mathrm{~Pa}\right)$ für 6 Stunden ausgeheizt (Silica-Alumina-Gel @ 448 K, 5A Zeolith @ 573 K). Die spezifischen Oberflächen der Materialien wurden mit der BET-Methode nach DIN ISO 9277 [67] bestimmt. Das Gesamtporenvolumen wurde aus der Stickstoffisotherme für einen Relativdruck $\mathrm{p} / \mathrm{p}_{0}=$ 0,98 mit der Methode nach Gurvich [68] ermittelt. Tabellen 3 und 4 geben einen Überblick über die strukturellen Charakteristika der Materialien. 
Tabelle 3: Strukturelle Eigenschaften der verwendeten Adsorbentien

\begin{tabular}{|c|c|c|}
\hline & Silica-Alumina-Gel & 5A Zeolith \\
\hline BET-Oberfläche [m²/g] & 737 & 568 \\
\hline Porenvolumen $\left[\mathrm{cm}^{3} / \mathrm{g}\right]$ & 0,49 & 0,35 \\
\hline \multicolumn{3}{|l|}{ Chemische Zusammensetzung (XRF) } \\
\hline $\mathrm{SiO}_{2}[\%]$ & ca. $97 \%$ & 47,7 \\
\hline $\mathbf{A l}_{2} \mathbf{O}_{3}[\%]$ & ca. $3 \%$ & 29,2 \\
\hline Sulfate [\%] & 0,06 & 0,04 \\
\hline $\mathrm{Na}[\%]$ & 0,05 & 2,6 \\
\hline $\mathrm{Ca}[\%]$ & - & 12,8 \\
\hline $\mathrm{K}[\%]$ & - & 0,4 \\
\hline Mg [\%] & - & 2,5 \\
\hline $\mathrm{Fe}[\%]$ & - & 0,87 \\
\hline Partikelgrößenverteilung [mm] & $2,5-3,5$ & $1,6-2,5$ \\
\hline
\end{tabular}

Tabelle 4: Strukturelle Eigenschaften der modifizierten Silica-Alumina-Gele

\begin{tabular}{|c|c|c|}
\hline & $\begin{array}{c}\text { Silica-Alumina-Gel } \\
\text { (ca. } 0,3 \text { Gew. } \% \mathrm{Al}_{2} \mathrm{O}_{3} \text { ) }\end{array}$ & $\begin{array}{c}\text { Silica-Alumina-Gel } \\
\text { (ca. 1,5 Gew.\% } \mathrm{Al}_{2} \mathrm{O}_{3} \text { ) }\end{array}$ \\
\hline BET-Oberfläche $\left[\mathrm{m}^{2} / \mathrm{g}\right]$ & 815 & 797 \\
\hline Porenvolumen $\left[\mathrm{cm}^{3} / \mathrm{g}\right]$ & 0,49 & 0,49 \\
\hline \multicolumn{3}{|l|}{ Chemische Zusammensetzung } \\
\hline $\mathrm{SiO}_{2}[\%]$ & ca. $99,7 \%$ & ca. $98,5 \%$ \\
\hline $\mathrm{Al}_{2} \mathbf{O}_{3}[\%]$ & ca. $0,3 \%$ & ca. $1,5 \%$ \\
\hline Partikelgrößenverteilung [mm] & $2,5-3,5$ & $2,5-3,5$ \\
\hline
\end{tabular}

Abbildung 14 zeigt die vermessenen Stickstoffisothermen bei $77 \mathrm{~K}$ aller eingesetzten Adsorbentien. 


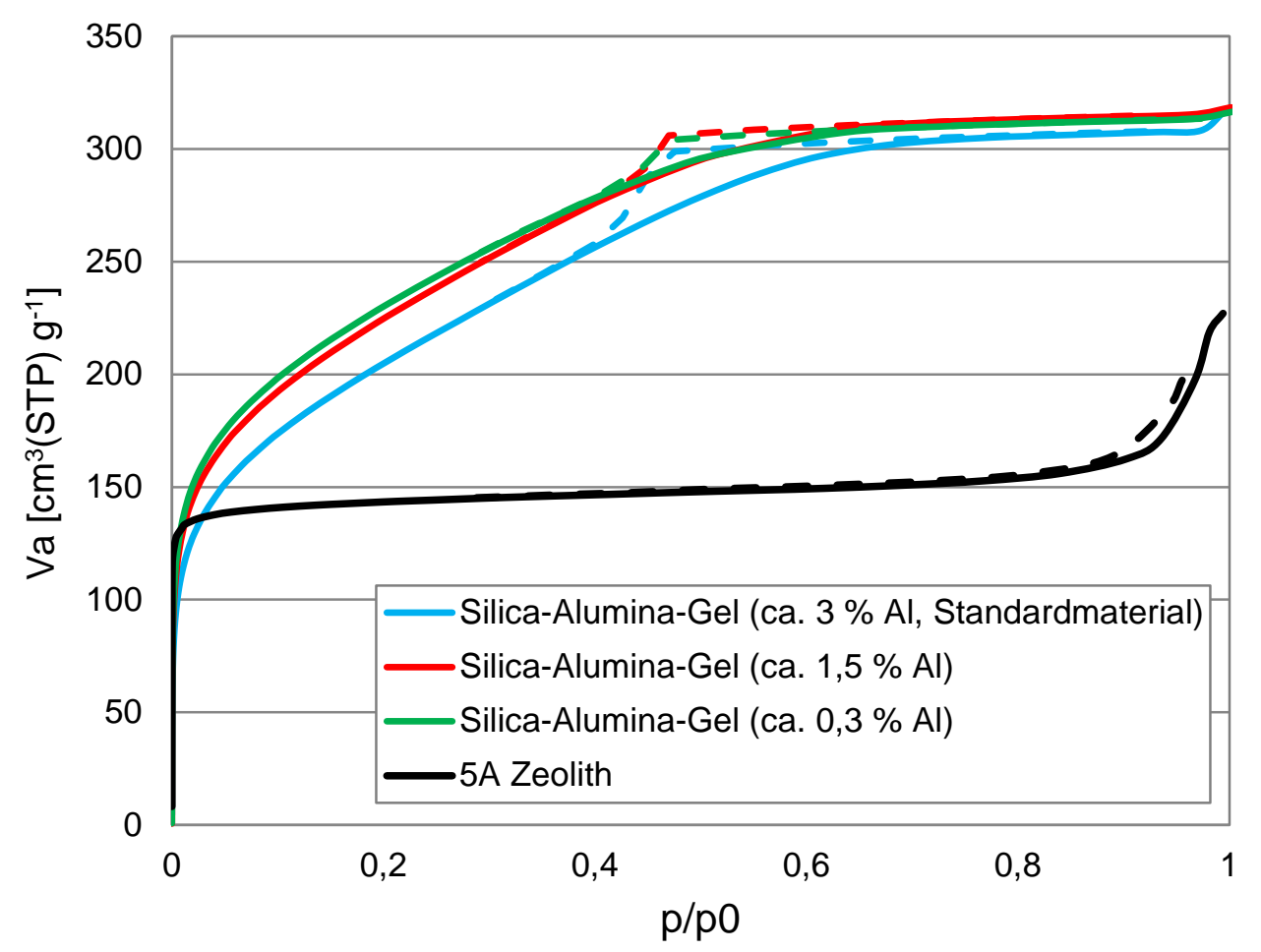

Abbildung 14: Stickstoffisothermen der verwendeten Adsorbentien bei $77 \mathrm{~K}$. Durchgezogene Linien: Adsorption; gestrichelte Linien: Desorption

Die Abbildung verdeutlicht, dass die drei Silica-Alumina-Gel-Varianten von ihren strukturellen Eigenschaften her sehr ähnlich sind. Die ermittelten Porenvolumina sind im Rahmen der Messgenauigkeit gleich. Die BET-Oberfläche wird mit fallendem Aluminium-Gehalt etwas größer. Die Stickstoffisotherme des 5A Zeolith zeigt einen charakteristischen LangmuirVerlauf mit Kapillarkondensation im sehr hohen Konzentrationsbereich.

\subsubsection{Adsorptive}

Die Untersuchungen umfassen Versuche mit verschiedenen organischen und anorganischen Schwefelverbindungen, Kohlendioxid und Wasser. Schwefelwasserstoff (99,5\%) wurde von Air Liquide Deutschland GmbH, Methylmercaptan (99,5 \%) und Carbonylsulfid (97,5\%) wurden von Sigma-Aldrich Chemie $\mathrm{GmbH}$ bezogen. Ethyl- und Propylmercaptan wurden als vorgemischte, zertifizierte Prüfgase von Air Liquide Deutschland $\mathrm{GmbH}$ zur Verfügung gestellt und entsprechend der in Abschnitt 3.2.3 geschilderten Vorgehensweise verwendet. Tabelle 5 gibt eine Auflistung physikalischer Eigenschaften aller verwendeten Adsorptive [38, $69,70,71,72,73,74]$. 
Tabelle 5: Physikalische Eigenschaften der verwendeten Adsorptive

\begin{tabular}{|l|l|c|c|c|c|}
\hline \multicolumn{1}{|c|}{ Verbindung } & Formel & $\begin{array}{c}\text { Molmasse } \\
{[\mathbf{g} / \mathbf{m o l}]}\end{array}$ & $\begin{array}{c}\text { Dampfdruck } \\
\text { bei } \mathbf{2 0}^{\circ} \mathbf{C}[\mathbf{b a r}]\end{array}$ & $\begin{array}{c}\text { Krit. Molekül- } \\
\text { durchmesser } \\
{\left[\mathbf{1 \mathbf { 0 } ^ { - 1 0 }} \mathbf{\text { m] }}\right.}\end{array}$ & $\begin{array}{c}\text { Dipolmoment } \\
{[\text { Debye] }}\end{array}$ \\
\hline Wasser & $\mathrm{H}_{2} \mathrm{O}$ & 18,015 & 0,023 & 2,6 & 1,84 \\
\hline Kohlendioxid & $\mathrm{CO}_{2}$ & 44,01 & 57,3 & 2,8 & 0 \\
\hline \multicolumn{5}{|}{ Schwefelverbindungen } \\
\hline Carbonylsulfid & $\mathrm{COS}$ & 60,07 & 11,3 & 2,8 & 0,72 \\
\hline Schwefelwasserstoff & $\mathrm{H}_{2} \mathrm{~S}$ & 34,08 & 18,2 & 3,6 & 0,98 \\
\hline Methylmercaptan & $\mathrm{CH}_{3} \mathrm{SH}$ & 48,11 & 1,7 & $3,8-4,5$ & 1,52 \\
\hline Ethylmercaptan & $\mathrm{C}_{2} \mathrm{H}_{5} \mathrm{SH}$ & 62,14 & 0,576 & 5,1 & 1,58 \\
\hline Propylmercaptan & $\mathrm{C}_{3} \mathrm{H}_{7} \mathrm{SH}$ & 76,16 & 0,165 & - & 1,60 \\
\hline
\end{tabular}

\subsubsection{Trägergase}

In allen Versuchen wurde die Adsorption der Zielkomponente aus dem Trägergas Methan (99,95 \%, Air Liquide Deutschland $\mathrm{GmbH}$ ) bestimmt. Die Adsorption von $\mathrm{H}_{2} \mathrm{O}$ wurde zu Vergleichszwecken zusätzlich aus dem Trägergas Stickstoff (99,99 \%, Air Liquide Deutschland $\mathrm{GmbH}$ ) gemessen. Tabelle 6 gibt eine Auflistung charakteristischer Stoffdaten der eingesetzten Trägergase.

Tabelle 6: Physikalische Eigenschaften der verwendeten Trägergase [38]

\begin{tabular}{|l|c|c|c|c|}
\hline \multicolumn{1}{|c|}{ Verbindung } & Formel & $\begin{array}{c}\text { Molmasse } \\
{[\mathbf{g} / \mathbf{m o l}]}\end{array}$ & $\begin{array}{c}\text { Siedepunkt bei } \\
\mathbf{1 , 0 1 3} \text { bar }\left[{ }^{\circ} \mathbf{C}\right]\end{array}$ & $\begin{array}{c}\text { Krit. Moleküldurch- } \\
\text { messer }\left[\mathbf{1 0 ^ { - 1 0 }} \mathbf{~ m} \text { ] }\right.\end{array}$ \\
\hline Stickstoff & $\mathrm{N}_{2}$ & 28,01 & $-195,79$ & 3,0 \\
\hline Methan & $\mathrm{CH}_{4}$ & 16,04 & -162 & 4,0 \\
\hline
\end{tabular}

\subsection{Messung von Durchbruchskurven}

\subsubsection{Rahmenbedingungen}

Alle im Rahmen dieser Arbeit durchgeführten Messreihen wurden bei einer Temperatur von $25^{\circ} \mathrm{C}$ und einem Absolutdruck von 1,3 bar durchgeführt.

Die Untersuchungen beschränken sich auf:

\section{i. Binäre Stoffsysteme}

Adsorption eines Adsorptivs aus einem Trägergas 


\section{ii. Ternäre Stoffsysteme}

Adsorption von zwei Adsorptiven aus einem Trägergas

\subsubsection{Versuchsanlage}

Im Rahmen der Arbeiten wurde eine Festbettversuchsanlage im Labormaßstab aufgebaut und in Betrieb genommen. Alle Adsorptionsuntersuchungen wurden an dieser Anlage durchgeführt. Abbildung 15 zeigt eine schematische Darstellung des Aufbaus. Abbildung 16 die Anlage im Laborbetrieb.

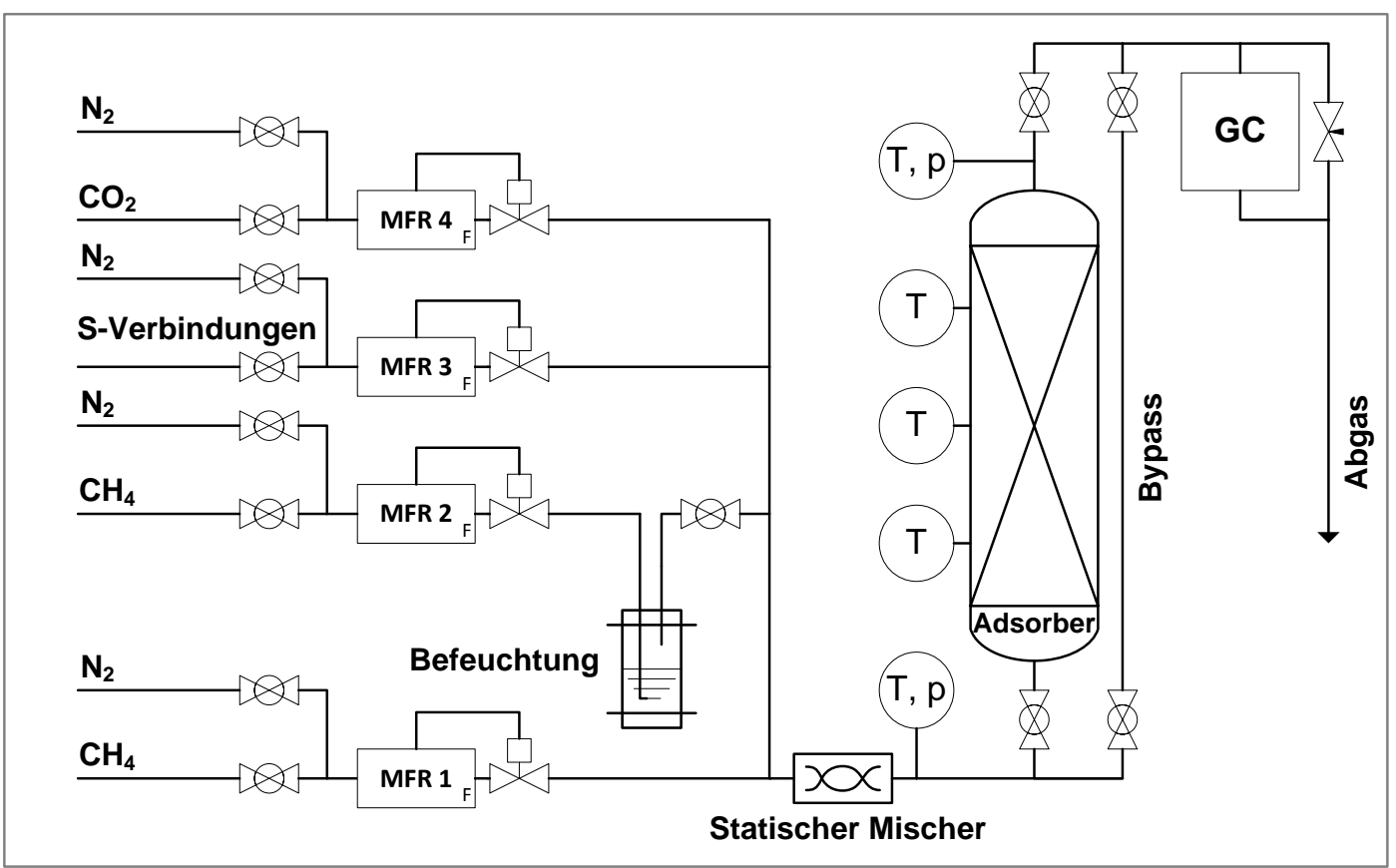

Abbildung 15: Schematische Darstellung der Versuchsanlage 


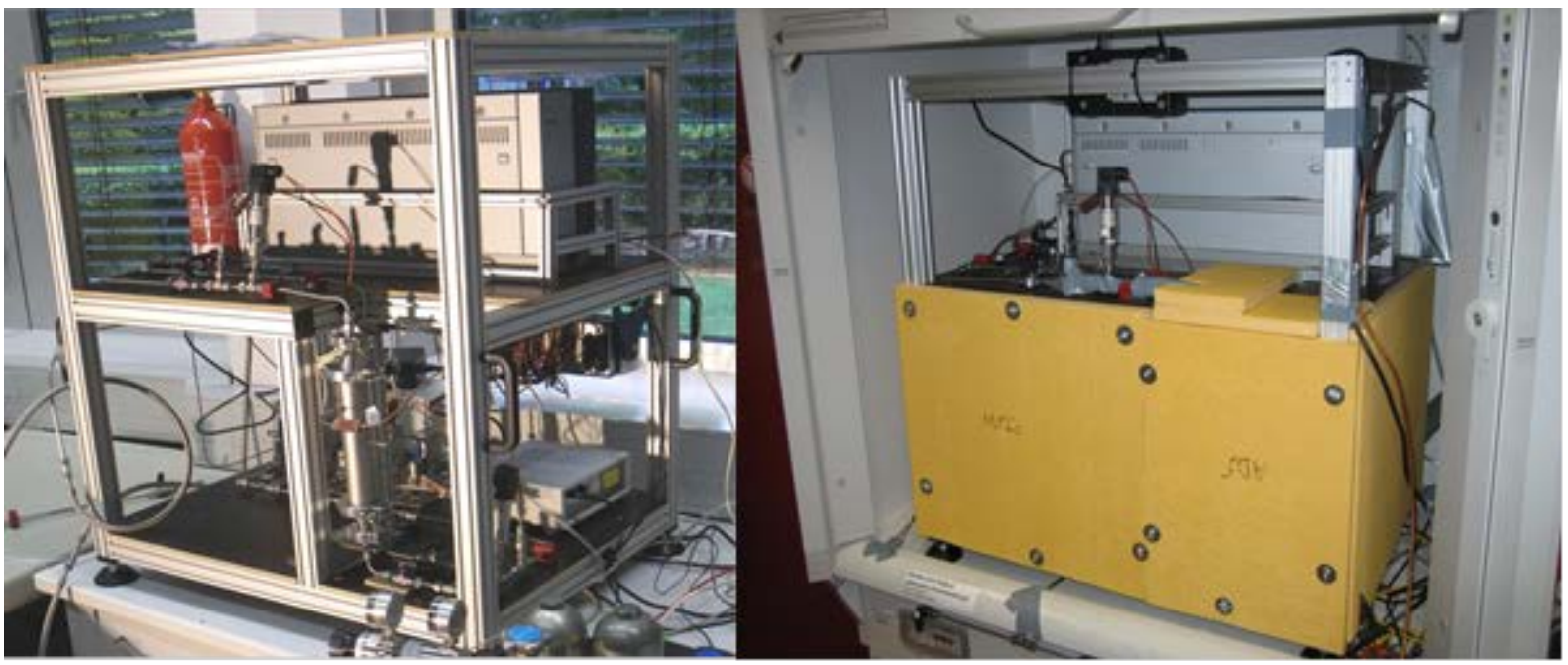

Abbildung 16: Versuchsanlage im Laborbetrieb

Die Anlage besteht aus drei Bereichen:

1. Gasmischsystem

2. Adsorberkolonne

3. Gasanalyse

\section{Gasmischsystem}

Die Versuchsgase wurden mit Hilfe thermischer Massenflussregler (MFR) aus Reingasen gemischt. Um in binären und ternären Gasmischungen Adsorptivkonzentrationen im ppmBereich zu erzeugen, wurden dem im Verhältnis sehr großen Trägergasvolumenstrom sehr geringe Volumenströme des Adsorptivgases zu dosiert. Tabelle 7 gibt Aufschluss über die Größenordnung der Volumenströme für eine Versuchsgasmischung im ppm-Bereich.

Tabelle 7: Beispielhaftes Volumenstromverhältnis für einen Versuch

\begin{tabular}{|l|l|}
\hline Gasmischung & Volumenströme \\
\hline Trägergas & $7,5 \mathrm{I}_{\mathrm{n}} / \mathrm{min}$ \\
\hline Adsorptiv $\left(\mathrm{H}_{2} \mathrm{~S}\right)$ & $15 \mathrm{~m} \mathrm{n}_{\mathrm{n}} / \mathrm{min}$ \\
\hline Resultierende Adsorptivkonzentration & ca. $2000 \mathrm{~mol} \mathrm{ppm}$ \\
\hline
\end{tabular}

Zur Erzeugung von Versuchsgasen mit den Komponenten Ethyl- und Propylmercaptan wurden zertifizierte Prüfgase mit genau definierter Konzentration bezogen und entsprechend der gewünschten Konzentration für das jeweilige Experiment verdünnt.

Für binäre und ternäre Versuche mit Wasser wurde ein geringer Volumenstrom durch flüssiges Wasser geleitet und auf diese Weise mit Wasser beladen. Die Einstellung der gewünschten Konzentration geschah wiederum durch eine Verhältnisregelung mit einem 
zweiten Volumenstrom. Für ternäre Untersuchungen mit Kohlendioxid und Schwefelwasserstoff wurden die entsprechenden Reingase über zwei thermische Massenflussregler in den Trägergasvolumenstrom dosiert.

Auf Grund des Gefährdungspotenzials durch das brennbare Trägergas Methan und die toxischen und korrosiven Schwefelverbindungen wurde die Gasversorgungsseite mit Inertgasspülleitungen versehen. Auf diese Weise lässt sich die Anlage im Stillstand vollständig mit Stickstoff durchspülen. Eine Anreicherung brennbarer oder toxischer Gase in der Anlage ist damit ausgeschlossen.

Im Anschluss an die Gasmischstrecke folgt ein statischer Mischer. Zwei aus Teflon bestehende, in das Leitungssystem integrierte statische Mischelemente sorgen für eine Homogenisierung der Gasmischung.

\section{Adsorberkolonne}

Die aus Edelstahl gebaute Adsorptionskolonne ist senkrecht angeordnet. Das Rohgas tritt im Experiment von der Unterseite in die Kolonne ein. Das adsorptiv gereinigte Produktgas verlässt den Adsorber an der Oberseite. Durch Ventilschaltungen kann das Versuchsgas durch eine Bypass-Leitung an der Kolonne vorbei geleitet werden. Die Überwachung des Adsorptionsprozesses erfolgt über Druck- und Temperaturüberwachung. Hierbei werden der Eintrittszustand vor dem Adsorber und der Austrittzustand hinter dem Adsorber erfasst. Mehrere Temperaturmessstellen, die in der Kolonne positioniert sind, geben Aufschluss über den Zustand der Schüttung während des Experiments. Der gesamte untere Aufbau der Versuchsanlage (Gasmischsystem, Adsorberbereich) wurde für die Experimente mit Hilfe eines Peltier Kühl-/Heizreglers auf 298 K thermostatisiert. Aus diesem Grund ist dieser Bereich vollständig isoliert (vgl. Abbildung 16 rechts).

Um Versuchszeiten zu optimieren und den Verbrauch an Gasen zu minimieren, wurden zwei unterschiedliche Adsorberdurchmesser verwendet. Bei der Dimensionierung ist zu beachten, dass eine Randgängigkeit des Gases vermieden wird. In der Literatur findet man als Auslegungskriterium zur Vermeidung von Randgängigkeitseffekten das Verhältnis von Adsorberdurchmesser/Partikeldurchmesser $D / d_{P}>10$ [38]. Tabelle 8 gibt Aufschluss über die Geometrie der Adsorber. 
Tabelle 8: Übersicht über die Geometrie der Adsorber

\begin{tabular}{|l|c|c|}
\hline Eigenschaft & Adsorber 1 & Adsorber 2 \\
\hline Höhe [cm] & 20 & 20 \\
\hline Durchmesser [cm] & 4 & 3 \\
\hline Max. Höhe/Durchmesser-Verhältnis & $5: 1$ & $6,6: 1$ \\
\hline Adsorbensfraktion [mm] & $2,5-3,5$ & $1,6-2,5$ \\
\hline Adsorberdurchmesser/Partikeldurchmesser-Verhältnis & 11,4 & 12 \\
\hline
\end{tabular}

\section{Gasanalyse}

Das aus der Kolonne austretende Produktgas wird quasi-kontinuierlich auf seine Zusammensetzung hin analysiert. Hierzu dient ein $\mu$-Gaschromatograph Mikro-GC Varian CP 4900, der je nach Retentionszeit der untersuchten Adsorptive Messzykluszeiten im Bereich von < 2 Minuten realisieren konnte. Auf Grund des geringen Prozessüberdrucks (1,3 bar Absolutdruck) wird der Gaschromatograph kontinuierlich mit Produktgas durchströmt. Ein automatisiertes Probenahmeventil gibt dem Gerät die Gasproben auf. Die Kalibrierung des Gerätes erfolgte mit zertifizierten Prüfgasen des Herstellers Air-Liquide Deutschland GmbH. Der verwendete Gaschromatograph ist ein Gerät mit mehreren GC-Kanälen. Das bedeutet, dass das automatisierte Probenahmeventil die Gasprobe auf bis zu drei Säulen aufgibt. Jede Säule kann je nach Trennaufgabe in gewünschtem Druck- und Temperaturprogramm verwendet werden und dementsprechend zur Auftrennung unterschiedlicher Verbindungen dienen. Allen Säulen ist als Detektor ein Wärmeleitfähigkeitsdetektor nachgeschaltet. Auf diese Weise können für eine Gasanalyse zeitgleich bis zu drei Chromatogramme erzeugt werden.

\subsubsection{Versuchsdurchführung}

Die Adsorbentien wurden zur Konditionierung nach Herstellerangaben für mindestens 12 Stunden im Trockenschrank ausgeheizt (5A Zeolith @ 573 K, Silica-Alumina-Gele @ 448 K). Die Experimente wurden wie folgt durchgeführt:

i. Vor dem Versuch wurde die Adsorberkolonne mit dem heißen Adsorbens befüllt.

ii. Anschließend wurde ein trockener Stickstoffvolumenstrom $\left(<1 \mathrm{ppm}_{\mathrm{V}} \mathrm{H}_{2} \mathrm{O}\right)$ auf die heiße Schüttung gegeben und es erfolgte ein Spülvorgang bis zum Erreichen des thermischen Gleichgewichts bei Adsorptionstemperatur (298 K). Um die Einflüsse der Koadsorption von Trägergasmolekülen oder Verunreinigungen des Trägergases für jedes Experiment vergleichbar zu machen, erfolgte vor jedem Versuch 
eine Spülung der Schüttung mit reinem Trägergas. Dieser Spülvorgang wurde wiederum bis zum Erreichen des thermischen Gleichgewichts bei $298 \mathrm{~K}$ durchgeführt.

iii. Im Anschluss an die Spülvorgänge wurde die Kolonne abgesperrt und der Gasstrom über eine Bypassleitung an der Kolonne vorbeigeleitet. Dann wurde das Versuchsgas erstellt. Über einen zweiten Massenflussregler wurde das Adsorptiv in das Trägergas dosiert. Die quasi-kontinuierliche Gasanalyse mit Hilfe des Gaschromatographen wurde gestartet.

iv. Nach Einstellung einer konstanten Zusammensetzung wurde die Gasmischung durch das Festbett geleitet und der aus der Kolonne austretende Produktgasstrom solange analysiert, bis wieder die zuvor eingestellte Feedkonzentration gemessen wurde und das Adsorbens vollständig beladen war.

\subsubsection{Weitere Geräte}

Die Gasmischungen für die Durchbruchsexperimente wurden mit thermischen Massenflussreglern des Herstellers Bronkhorst High Tech aus Reingasen erstellt. Die Wasserkonzentration wurde mit einem Testo 6615 Polymer-Taupunktsensor gemessen. Der Anlagendruck wurde am Adsorberein- und austritt mit Bd Sensors DMP 331i Druckmessumformern überwacht. Die Temperatur wurde am Adsorberein- und -austritt und an mehreren Stellen innerhalb der Kolonne mit Hilfe von Thermoelementen des Typs T erfasst. Alle medienberührten Anlagenbauteile wie Rohrleitungen, Adsorberkolonne und Ventile wurden zur Passivierung der Oberflächen mit der Beschichtung SilcoNert ${ }^{T M} 2000$ von SilcoTek versehen. Alle Messwerte wurden mit Hardware des Herstellers National Instruments erfasst. Die Prozessüberwachung und das Speichern der Daten wurde mit der Software LabVIEW realisiert.

\subsection{Massenbilanz über der Schüttung}

Die im Rahmen dieser Arbeit vorgestellten Adsorptionsgleichgewichte werden aus Durchbruchskurvenmessungen bestimmt. Abbildung 17 soll dies schematisch verdeutlichen. Im Versuch wird die Schüttung durch eine Ventilschaltung mit dem Versuchsgas beaufschlagt. Findet eine Adsorption statt, werden Adsorptivmoleküle gebunden. In der Produktgasanalyse äußert sich dies durch eine Adsorptivkonzentration, die unterhalb der aufgegebenen Feedkonzentration liegt. Der Versuch wird solange durchgeführt bis sich Produktgaskonzentration 
und Feedkonzentration angeglichen haben $\left(\mathrm{y}_{\text {ein }}=\mathrm{y}_{\text {aus }}\right)$ und ein eventuell auftretender Temperaturanstieg in der Schüttung wieder abgeklungen ist. Das System befindet sich dann im thermodynamischen Gleichgewicht. Mit Kenntnis der eingesetzten Adsorbensmasse und des aufgegebenen Stoffmengenstroms lässt sich die Adsorbensbeladung durch eine Integration der Fläche über der Durchbruchskurve ermitteln.

Durchbruchskurve:

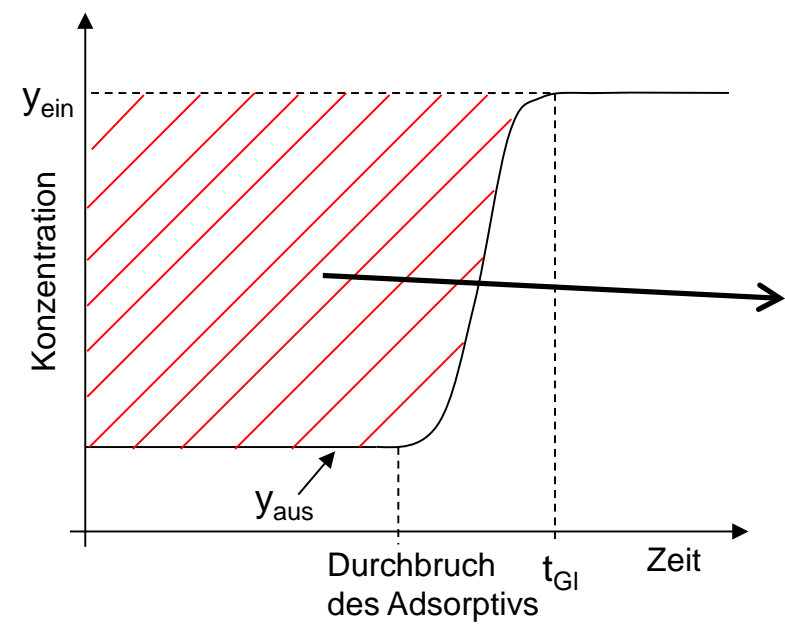

Adsorptionsisotherme:

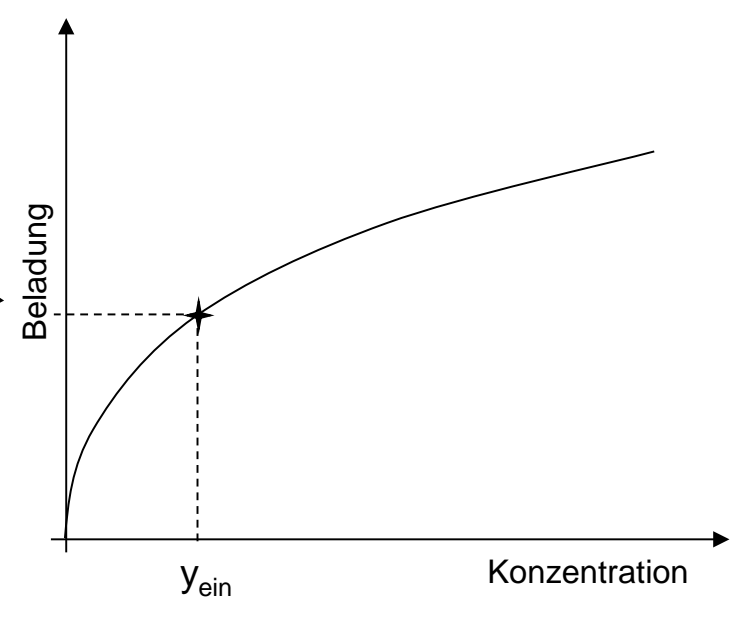

Abbildung 17: Schematische Darstellung der Bestimmung der Adsorbensbeladung

Aus der Massenbilanz um den Adsorber ergibt sich für den Fall eines binären Gasgemisches, bei dem eine Komponente adsorbiert (eine Trägergasadsorption kann auf Grund des Spülvorgangs mit Trägergas ausgeschlossen werden, vgl. Abschnitt „Versuchsdurchführung"), folgende Beziehung:

$$
X=\frac{\dot{n}_{F I}}{m_{\text {Ads }}} \int_{t_{0}}^{t_{\text {Gl }}}\left(\frac{y_{\text {ein }}-y_{\text {aus }}}{1-y_{\text {aus }}}\right) d t
$$

Bei dieser Betrachtung entspricht $\dot{n}_{F I}$ dem Gesamtstoffmengenstrom, $\mathrm{m}_{\mathrm{Ads}}$ der eingesetzten Adsorbensmasse, $y_{\text {ein }}$ der Rohgaskonzentration und $y_{\text {aus }}$ der jeweils gemessenen Produktgaskonzentration.

Für den ternären Fall, also einer Adsorption von zwei Komponenten aus einem sehr schwach adsorbierenden Trägergas, errechnet sich die Beladung für Komponente 1 nach:

$$
X_{1}=\frac{\dot{n}_{F I}}{m_{\text {Ads }}} \int_{t_{0}}^{t_{\mathrm{Gl}}}\left(y_{1, \text { ein }}-\left(y_{1, \text { aus }} \frac{1-y_{1, \text { ein }}-y_{2, \text { ein }}}{1-y_{1, \text { aus }}-y_{2, \text { aus }}}\right)\right) d t
$$


Für jedes untersuchte Stoffsystem wurden im vorgesehenen Untersuchungsbereich zwischen 8-12 Durchbruchskurvenmessungen bei verschiedenen Adsorptivkonzentrationen durchgeführt. Tabelle 9 gibt Aufschluss über den Untersuchungsbereich der jeweiligen Adsorptive:

Tabelle 9: Untersuchungsbereiche der Adsorptive

\begin{tabular}{|l|c|}
\hline Adsorptiv & Untersuchungsbereich \\
\hline $\mathrm{H}_{2} \mathrm{~S}$ & bis $2000 \mathrm{~mol} \mathrm{ppm}$ \\
\hline $\mathrm{COS}$ & bis $2000 \mathrm{~mol} \mathrm{ppm}$ \\
\hline $\mathrm{CH}_{3} \mathrm{SH}$ & bis $2000 \mathrm{~mol} \mathrm{ppm}$ \\
\hline $\mathrm{C}_{2} \mathrm{H}_{5} \mathrm{SH}$ & bis $2000 \mathrm{~mol} \mathrm{ppm}$ \\
\hline $\mathrm{C}_{3} \mathrm{H}_{7} \mathrm{SH}$ & bis $450 \mathrm{~mol} \mathrm{ppm}$ \\
\hline $\mathrm{H}_{2} \mathrm{O}$ & bis $2000 \mathrm{~mol} \mathrm{ppm}$ \\
\hline $\mathrm{CO}_{2}$ & bis $50000 \mathrm{~mol} \mathrm{ppm}$ \\
\hline
\end{tabular}

Abbildung 18 zeigt beispielhaft Durchbruchskurven für das Stoffsystem $\left(\mathrm{CO}_{2}\right.$ in $\mathrm{CH}_{4}$ an $5 \mathrm{~A}$ Zeolith).

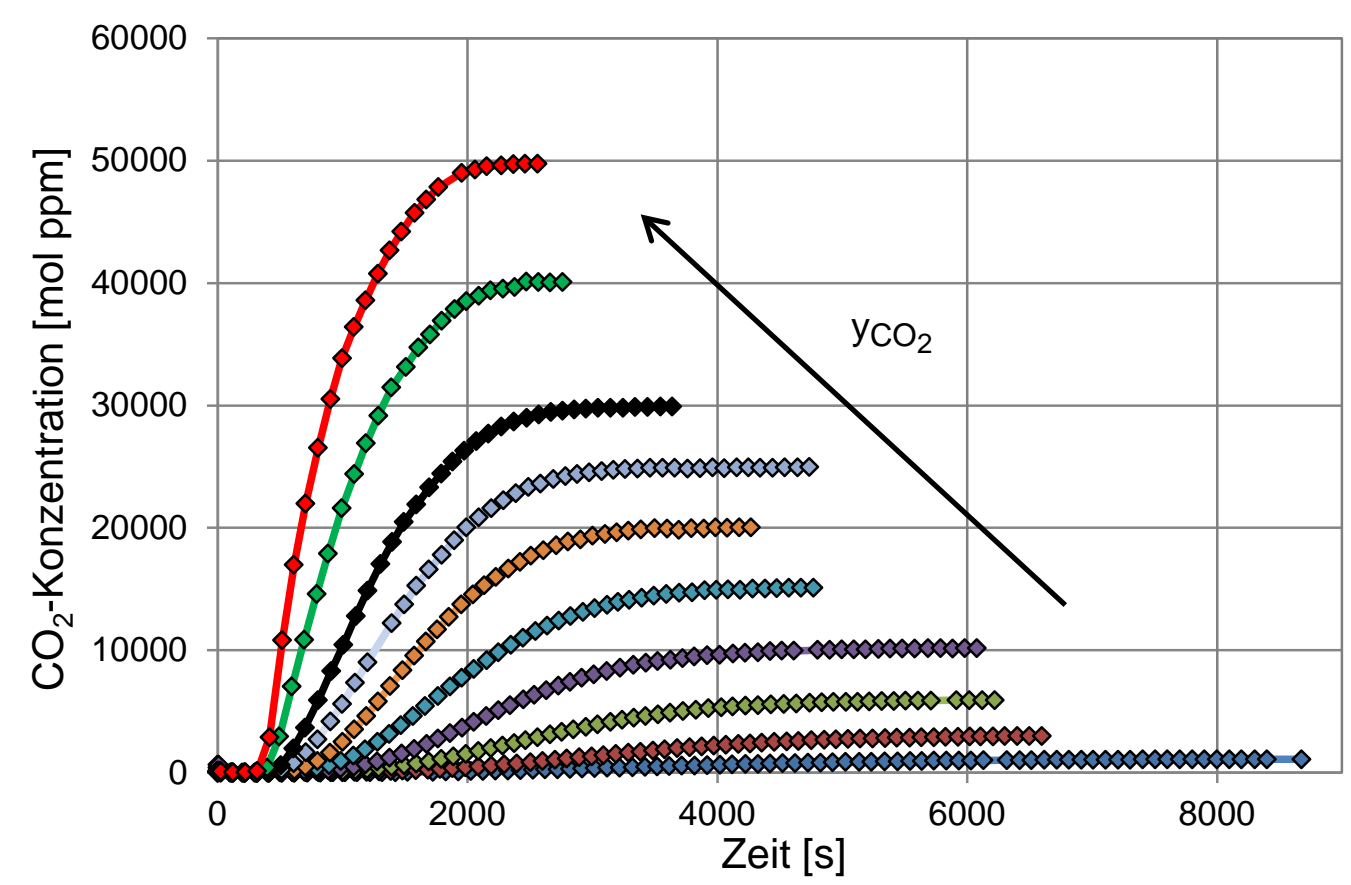

Abbildung 18: Experimentell bestimmte Durchbruchskurven $\left(\mathrm{CO}_{2}\right.$ in $\mathrm{CH}_{4} / 5 \mathrm{~A}$ Zeolith $)$

Abbildung 18 verdeutlicht die Abhängigkeit der Durchbruchszeit von der AdsorptivKonzentration. Durch eine größere Adsorptiv-Stoffmenge in der Gasmischung ergibt sich eine schnellere Absättigung des Festbettes. Eine vertiefte Diskussion der Durchbruchskurven erfolgt in Kapitel 4. 


\subsection{Approximation der Messwerte}

Die auf die oben geschilderte Weise ermittelten Beladungen ließen sich mit Hilfe bekannter Isothermengleichungen beschreiben. Dabei zeigten sich für die untersuchten Stoffsysteme auf dem 5A Zeolith im Untersuchungsbereich durchweg Verläufe, die mit sehr guter Näherung durch die Langmuir-Gleichung 3.3 der Form

$$
x=x_{\text {mon }} \frac{b \cdot y}{1+b \cdot y}
$$

beschrieben werden können. Die Anpassung erfolgte mittels bekannter Methoden [75]. Für das Silica-Alumina-Gel ließen sich die Messwerte durch die Henry-Gleichung 3.4 oder durch die Freundlich-Gleichung 3.5 beschreiben:

$$
\begin{aligned}
& X=k_{H} \cdot y \\
& X=k_{F} \cdot y^{n}
\end{aligned}
$$

Zur Beschreibung der Anpassungsgüte der Messwerte an die verwendeten Isothermengleichungen wurde das Bestimmtheitsmaß nach Gleichung 3.6

$$
R^{2}=1-\frac{\sum_{i=1}^{n}\left(X_{\text {meas }, i}-X_{c a l c, i}\right)^{2}}{\sum_{i=1}^{n}\left(X_{\text {meas }, i}-X_{\text {mean }, i}\right)^{2}}
$$

herangezogen.

\subsection{Fehlerrechnung}

Die Berechnung der Adsorbensbeladung nach der geschilderten Auswertungsmethode ist fehlerbehaftet. Entsprechend der Massenbilanz 3.1 ist die Beladung von drei Messgrößen abhängig:

1. Konzentrationsmessung

2. Messung/Regelung des Stoffmengenstroms

3. Messung der Adsorbensmasse

Die Zeitmessung wird für die Bilanzierung als nicht fehlerbehaftete Größe angenommen und ist daher zu vernachlässigen. 
Die maximale Messunsicherheit setzt sich aus systematischem und statistischem Fehler zusammen. Systematische Fehler sind Unsicherheiten, die z. B. durch eine fehlerbehaftete Kalibrierung eines Messgerätes oder ein nichtlineares Verhalten eines Messgerätes entstehen. Tabelle 10 gibt eine Auflistung sämtlicher Fehlerquellen für die Bestimmung der Adsorbensbeladung an.

Tabelle 10: Fehler der verwendeten Messgeräte $[76,77,78]$

\begin{tabular}{|l|l|l|l|}
\hline Messgröße & Messgerät & $\begin{array}{l}\text { Systematischer } \\
\text { Fehler }\end{array}$ & $\begin{array}{l}\text { Statistischer } \\
\text { Fehler }\end{array}$ \\
\hline Adsorptivkonzentration & $\mu$-GC & $\pm 3 \%$ (Kalibrierfehler) & $\begin{array}{l}\text { Reproduzierbarkeit: } \\
\leq 3 \% \text { (Erfahrungs- } \\
\text { wert) }\end{array}$ \\
\hline Stoffmengenstrom & $\begin{array}{l}\text { Thermische Massen- } \\
\text { flussregler }\end{array}$ & $\begin{array}{l} \pm 0,5 \% \\
\text { vom Messwert } \\
\pm 0,1 \% \text { vom Endwert }\end{array}$ & $\begin{array}{l}\text { Reproduzierbarkeit: } \\
0,2 \% \text { vom Messwert }\end{array}$ \\
\hline Adsorbensmasse & Waage (Acculab 3100.2) & $\begin{array}{l} \pm 0,02 \text { g Linearität } \\
\pm 0,0002 \text { g Linearität }\end{array}$ & $\begin{array}{l}\text { Reproduzierbarkeit: } \\
0,01 \text { g } \\
\text { Reproduzierbarkeit: } \\
\pm 0,0001 \mathrm{~g}\end{array}$ \\
\hline
\end{tabular}

Den für die Beschreibung des systematischen Fehlers wesentlichen Beitrag liefert der Kalibrierfehler des Konzentrationsmessgerätes. Dieser entsteht durch die Verwendung von Kalibriergasen, die ebenfalls eine Unsicherheit besitzen. Der systematische Fehler durch die Stoffmengenstrommessung ist deutlich geringer. Derjenige der Waagen ist für die verwendeten Adsorbenseinwaagen sehr gering und daher vernachlässigbar. Da alle Untersuchungen bei gleichen Stoffmengenströmen und ähnlichen Adsorbenseinwaagen durchgeführt wurden variiert der systematische Fehler nicht stark. Der relative systematische Fehler der Untersuchungen liegt im Bereich von $\pm 3,63 \%$. Er ergibt sich aus der Addition der systematischen Einzelfehler:

$$
\left(\frac{\Delta X}{X}\right)=\left(\frac{\Delta \dot{n}}{\dot{n}_{F I}}\right)+\left(\frac{\Delta y}{y}\right)
$$

Tabelle 11 zeigt bespielhaft für einen Versuch mit üblichem Stoffmengenstrom die berechneten relativen systematischen Fehler.

Tabelle 11: Beispielhafte Berechnung des relativen systematischen Gesamtfehlers

\begin{tabular}{|l|c|c|}
\hline Messgröße & Üblicher Stoffmengenstrom & Relativer systematischer Fehler \\
\hline Konzentration & - & $3 \%$ \\
\hline Stoffmengenstrom & $5,59 * 10^{-3} \mathrm{~mol} / \mathrm{s}$ & $0,63 \%$ \\
\hline Gewicht & - & vernachlässigt \\
\hline & $\begin{array}{r}\text { Relativer systematischer } \\
\text { Gesamtfehler }\end{array}$ & $\pm \mathbf{3 , 6 3} \%$ \\
\cline { 2 - 3 } & & \\
\end{tabular}


Ein statistischer Fehler oder auch zufälliger Fehler besteht durch unvorhersehbare äußere Einflüsse. Eine Abschätzung des statistischen relativen Gesamtfehlers gelingt unter Zuhilfenahme der Gauß'schen Fehlerfortpflanzung der allgemeinen Form

$$
\begin{gathered}
G=F\left(X_{1}, X_{2}, \ldots \ldots, X_{n}\right) \\
\Delta G=\sqrt{\left(\frac{\partial G}{\partial X_{1}} \Delta X_{1}\right)^{2}+\left(\frac{\partial G}{\partial X_{2}} \Delta X_{2}\right)^{2}+\ldots \ldots \ldots+\left(\frac{\partial G}{\partial X_{n}} \Delta X_{n}\right)^{2}}
\end{gathered}
$$

Da nicht zu jeder Zeit ein Messwert zur Verfügung steht, wird die Integration der Fläche über der Durchbruchskurve numerisch durchgeführt. Hierbei ist für die Summation der einzelnen Flächeninkremente die Flächenkorrektur gemäß des in Abbildung 19 dargestellten Schemas zu berücksichtigen.

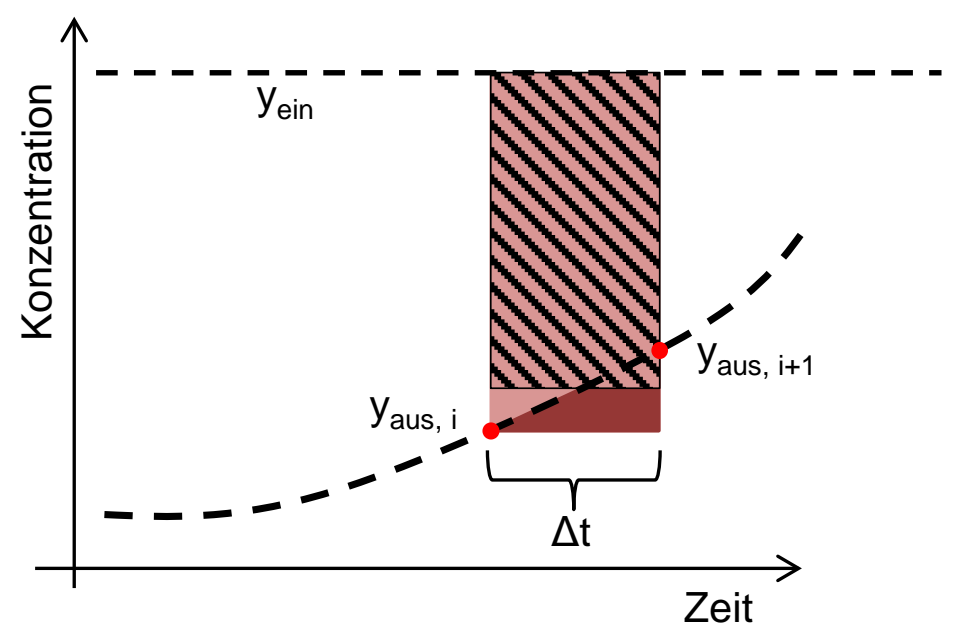

Abbildung 19: Schematische Darstellung der Inkrementberechnung der Durchbruchskurve

Dazu muss zur Berechnung eines Inkrements (schraffierte Fläche) der Mittelwert der gemessenen Austrittskonzentration verwendet werden:

$$
y_{\text {aus }}=\frac{y_{\text {aus }, i+1}+y_{\text {aus }, i}}{2}
$$

Damit ergibt sich für die Beladung durch Summation der Inkremente:

$$
X=\frac{\dot{n}_{F I}}{m_{\text {Ads }}} \sum\left(\frac{y_{\text {ein }}-y_{\text {aus }}}{1-y_{\text {aus }}}\right) \Delta t
$$

Für Untersuchungen im Spurenbereich lässt sich eine Änderung des Stoffmengenstroms infolge der Adsorption vernachlässigen. Gleichung 3.11 lässt sich daher zu 


$$
X=\frac{\dot{n}_{F 1}}{m_{\text {Ads }}} \sum\left(y_{\text {ein }}-y_{\text {aus }}\right) \Delta t
$$

vereinfachen.

Geht man von einem über den betrachteten Konzentrationsbereich gleichbleibenden statistischen Fehler der Konzentrationsmessung aus, kann für die Konzentrationsmessung $y_{\text {aus }}$ eine über den Versuchszeitraum gemittelte Ausgangskonzentration $\bar{y}_{\text {aus }}$ angenommen werden. Daraus ergibt sich auch eine mittlere Konzentrationsdifferenz. Für N Messungen gilt dann:

$$
\sum_{i=1}^{N}\left(y_{\text {ein }}-\bar{y}_{\text {aus }}\right)=N \cdot\left(y_{\text {ein }}-\bar{y}_{\text {aus }}\right)=N \cdot \Delta \bar{y}
$$

Mit $\Delta t=\frac{t}{N}$ ergibt sich für die Beladung:

$$
X=\frac{\dot{n}_{F I}}{m_{A d s}} \cdot \Delta \bar{y} \cdot t
$$

Um für diesen Term die Gauß'sche Fehlerfortpflanzungsformel gemäß Gleichung 3.9 anwenden zu können, müssen zunächst die partiellen Ableitungen nach den Variablen $\Delta \bar{y}, \dot{n}_{F /}$ und $m_{\text {Ads }}$ gebildet werden.

$$
\begin{gathered}
\frac{\partial X}{\partial \Delta \bar{y}}=\frac{\dot{n}_{F I}}{m_{A d s}} \cdot t, \frac{\partial X}{\partial m_{A d s}}=-\frac{\dot{n}_{F I}}{m_{A d s}^{2}} \cdot \Delta \bar{y} \cdot t, \frac{\partial X}{\partial \dot{n}_{F l}}=\frac{1}{m_{A d s}} \cdot \Delta \bar{y} \cdot t \\
\Delta X=\sqrt{\left(\frac{\dot{n}_{F l}}{m_{A d s}} \cdot t \cdot(\Delta \Delta \bar{y})\right)^{2}+\left(-\frac{\dot{n}_{F l}}{m_{A d s}^{2}} \cdot \Delta \bar{y} \cdot t \cdot\left(\Delta m_{A d s}\right)\right)^{2}+\left(\frac{1}{m_{A d s}} \cdot \Delta \bar{y} \cdot t \cdot\left(\Delta \dot{n}_{F I}\right)\right)^{2}}
\end{gathered}
$$

Für den relativen statistischen Fehler ergibt sich

$$
\frac{\Delta X}{X}=\sqrt{\left(\frac{\Delta \Delta \bar{y}}{\Delta \bar{y}}\right)^{2}+\left(\frac{\Delta m_{A d s}}{m_{A d s}}\right)^{2}+\left(\frac{\Delta \dot{n}_{F I}}{\dot{n}_{F I}}\right)^{2}}
$$

Mit den unter Tabelle 10 gegebenen Reproduzierbarkeiten ergeben sich für die durchgeführten Untersuchungen relative statistische Fehler im Bereich von $\leq \pm 3,01 \%$.

Größtmögliche Fehler für die Untersuchungsmethode ergeben sich durch Addition des systematischen und statistischen Fehlers und liegen im Bereich von $\leq \pm$ 6,6 \% 
Anhand der Massenbilanzierung erkennt man zudem, dass alle Messgrößen, die zur Ermittlung der Adsorbensbeladungen benötigt werden, als weitestgehend unabhängig von Systemtemperatur und -druck betrachtet werden können. Die Lage des thermodynamischen Gleichgewichts der Adsorption ist jedoch bestimmt durch Temperatur und Druck. Aus diesem Grund haben diese beiden Größen einen Einfluss auf die Adsorbensbeladung. Zur Temperaturregelung wurde ein Luft-Luft Peltier Heiz-/Kühlaggregat verwendet. Das Gerät arbeitete mit einer Regelgenauigkeit von $\pm 0,5 \mathrm{~K}$. Die verwendeten Drucktransmitter weisen eine Genauigkeit von \pm 2 mbar auf (0,1\% von 2000 mbar FSO).

\section{Überprüfung der Messsignale und Reproduzierbarkeit}

Die Konzentrationsmessung mittels gaschromatographischer Methode kann auf Grund verschiedener Einflüsse Schwankungen unterliegen. Im Laufe der Zeit können sich z. B. die in den Säulen befindlichen stationären Phasen verändern. Diese Veränderungen können sich in veränderten Wechselwirkungen zwischen stationärer Phase und dem zu trennenden Gasgemisch äußern. Daraus können sich veränderte Retentionszeiten ergeben, was zu fehlerhaft ausgewerteten Chromatogrammen führen kann. Um derartige Einflüsse auszuschließen, wurde der Gaschromatograph vor jeder Messreihe auf die jeweilige Verbindung bei verschiedenen Konzentrationen neu kalibriert.

Die korrekte Messung und Regelung der thermischen Massenflussregler wurde regelmäßig durch Vergleichsmessung mit einem Gilibrator (Seifenblasen-Strömungsmesser) des Herstellers SENSIDYNE überprüft. Die Gewichtsmessung der verwendeten Waagen wurde durch Eichgewichte kontrolliert.

Die Reproduzierbarkeit der Untersuchungsmethode wurde in regelmäßigen Abständen durch Wiederholungsmessungen überprüft. Da das Verfahren weitestgehend automatisiert abläuft, sind äußere Einflüsse wie z. B. eine Streuung der Messwerte auf Grund verschiedener Anlagenbediener ausgeschlossen. Die sehr gute Reproduzierbarkeit der Untersuchungsmethode soll im Folgenden an beispielhaften Widerholungsmessungen verdeutlicht werden. 
Tabelle 12: Beispielhafte Wiederholungsmessung für das binäre Stoffsystem $\mathrm{H}_{2} \mathrm{~S}$ in $\mathrm{CH}_{4}$

\begin{tabular}{|l|c|c|}
\hline \multicolumn{1}{|c|}{ Versuch } & $\begin{array}{c}\text { Konzentration } \\
{[\mathbf{m o l} \mathbf{~ p p m ]}}\end{array}$ & $\begin{array}{c}\text { Beladung } \\
{[\mathbf{m o l} / \mathbf{k g}]}\end{array}$ \\
\hline Messung 1 & 2064 & 0,02586 \\
\hline Messung 2 & 2069 & 0,02609 \\
\hline Messung 3 & 2066 & 0,02603 \\
\hline Mittelwert $\bar{X}$ & 2066,33 & 0,02599 \\
\hline Streuung s & 2,51 & 0,0000283 \\
\hline $\begin{array}{l}\text { Vertrauensbereich } \Delta \bar{x} \\
\text { (Konfidenzniveau 95\%: } \mathrm{t}=2,353 \text { für } \mathrm{n}=3 \text { ) }\end{array}$ & 3,41 & 0,00003844 \\
\hline Rel. Abweichung $\frac{\Delta \bar{x}}{\bar{x}}$ & $\mathbf{0 , 1 6 5 \%}$ & $\mathbf{0 , 1 4 7} \%$ \\
\hline
\end{tabular}




\section{$4 \quad$ Experimentelle Ergebnisse und Diskussion}

\subsection{Binäre Stoffsysteme}

\subsection{1 Überblick}

Das folgende Kapitel umfasst die ermittelten Adsorptionsisothermen der untersuchten binären Stoffsysteme. Die Darstellungen der Isothermen zeigen als Auftragung die Beladung des Adsorbens (mol/kg) (y-Achse) über der Gleichgewichtskonzentration des Adsorptivs (mol ppm) in der Gasphase (x-Achse). Die Symbole bezeichnen die aus den gemessenen Durchbruchskurven berechneten Kapazitäten. Die zugehörigen Linien stellen die angepassten Adsorptionsisothermen dar. Es sind Messreihen für die Adsorption von $\mathrm{CO}_{2}, \mathrm{COS}, \mathrm{H}_{2} \mathrm{~S}$, $\mathrm{CH}_{3} \mathrm{SH}, \mathrm{C}_{2} \mathrm{H}_{5} \mathrm{SH}, \mathrm{C}_{3} \mathrm{H}_{7} \mathrm{SH}$ und $\mathrm{H}_{2} \mathrm{O}$ aus dem Trägergas $\mathrm{CH}_{4}$ dargestellt. Zusätzlich wurde die Adsorption von $\mathrm{H}_{2} \mathrm{O}$ aus dem Trägergas $\mathrm{N}_{2}$ untersucht.

\subsubsection{Adsorptionsisothermen an Silica-Alumina-Gel}

Für das Silica-Alumina-Gel wurden insgesamt 7 binäre Stoffsysteme untersucht. Abbildung 20 zeigt die Isothermen der untersuchten Systeme. 


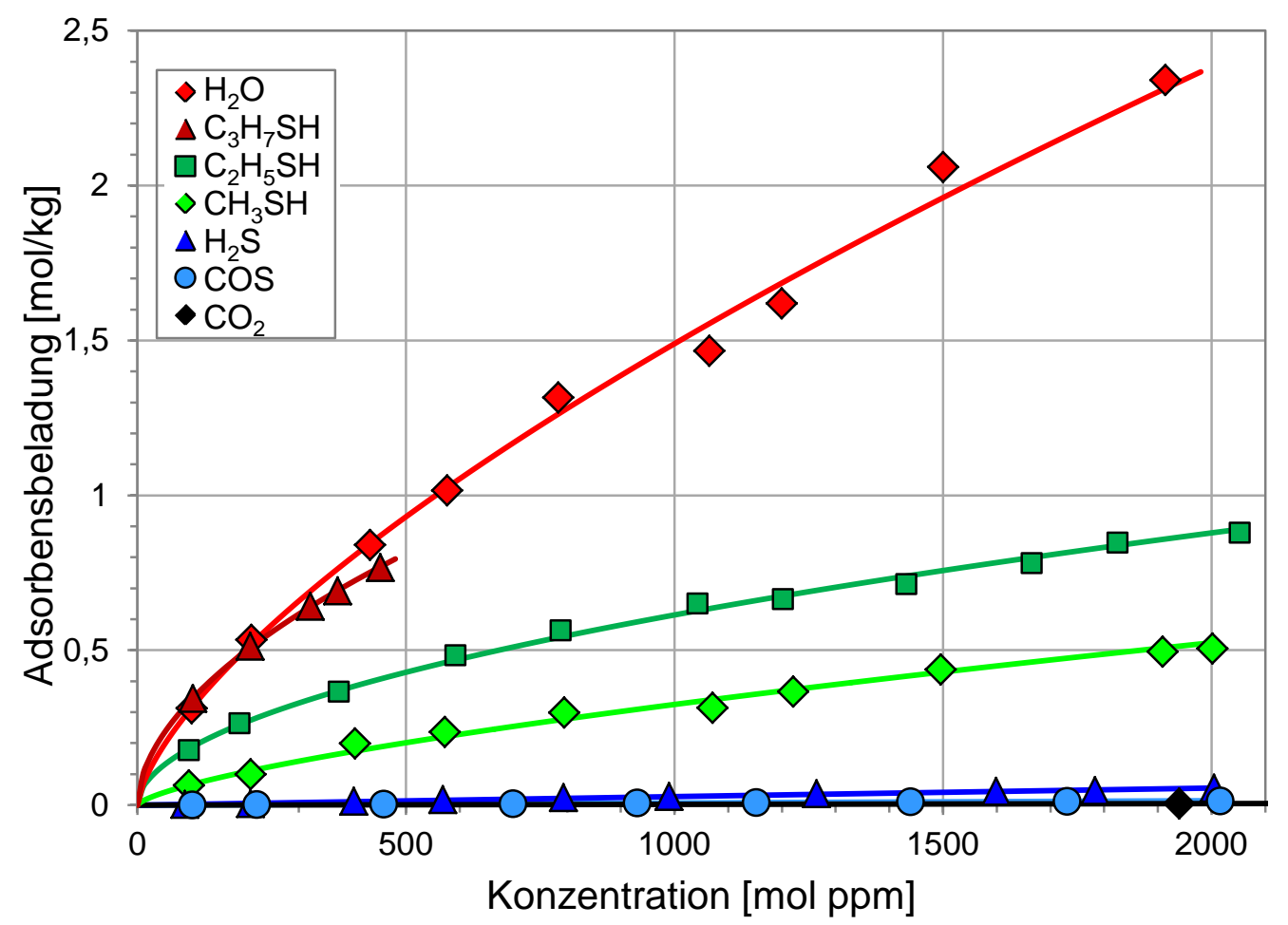

Abbildung 20: Isothermen für die Adsorption verschiedener Schwefelverbindungen, $\mathrm{CO}_{2}$ und $\mathrm{H}_{2} \mathrm{O}$ aus $\mathrm{CH}_{4}$ am Silica-Alumina-Gel

Es sind deutliche Unterschiede in den ermittelten Adsorptionskapazitäten zu verzeichnen. Im Folgenden werden die verschiedenen Adsorptive bei einer Konzentration von $450 \mathrm{~mol}$ ppm verglichen, da Propylmercaptan auf Grund apparativer Gegebenheiten lediglich bis zu einer Konzentration von $450 \mathrm{~mol}$ ppm in der Gasmischung betrachtet wurde (vgl. Abschnitt 3.2.3).Die stärkste Adsorption im dargestellten Untersuchungsbereich zeigt $\mathrm{H}_{2} \mathrm{O}$ mit ca. 0.85 $\mathrm{mol} / \mathrm{kg}$ für eine Konzentration im Bereich von $450 \mathrm{~mol}$ ppm. Darauf folgen Propyl-, Ethyl- und Methylmercaptan mit geringeren Kapazitäten im Bereich 0.7-0.2 mol/kg. Es zeigen sich für Propylmercaptan erwartungsgemäß in diesem vergleichsweise geringen Konzentrationsbereich deutlich größere Beladungskapazitäten als für die kürzeren Homologen. Das Verhalten von $\mathrm{H}_{2} \mathrm{O}$ und der Mercaptane kann in sehr guter Näherung durch die Freundlich-Gleichung wiedergegeben werden. Die schwach polaren Adsorptive $\left(\mathrm{H}_{2} \mathrm{~S}, \mathrm{COS}, \mathrm{CO}_{2}\right)$ zeigen Beladungen im Bereich der nächstgeringeren Größenordnung. Innerhalb dieser drei Verbindungen bestehen ebenfalls starke Unterschiede in den ermittelten Kapazitäten. Zur genaueren Darstellung sind diese Isothermen in Abbildung 21 im Detail dargestellt. 


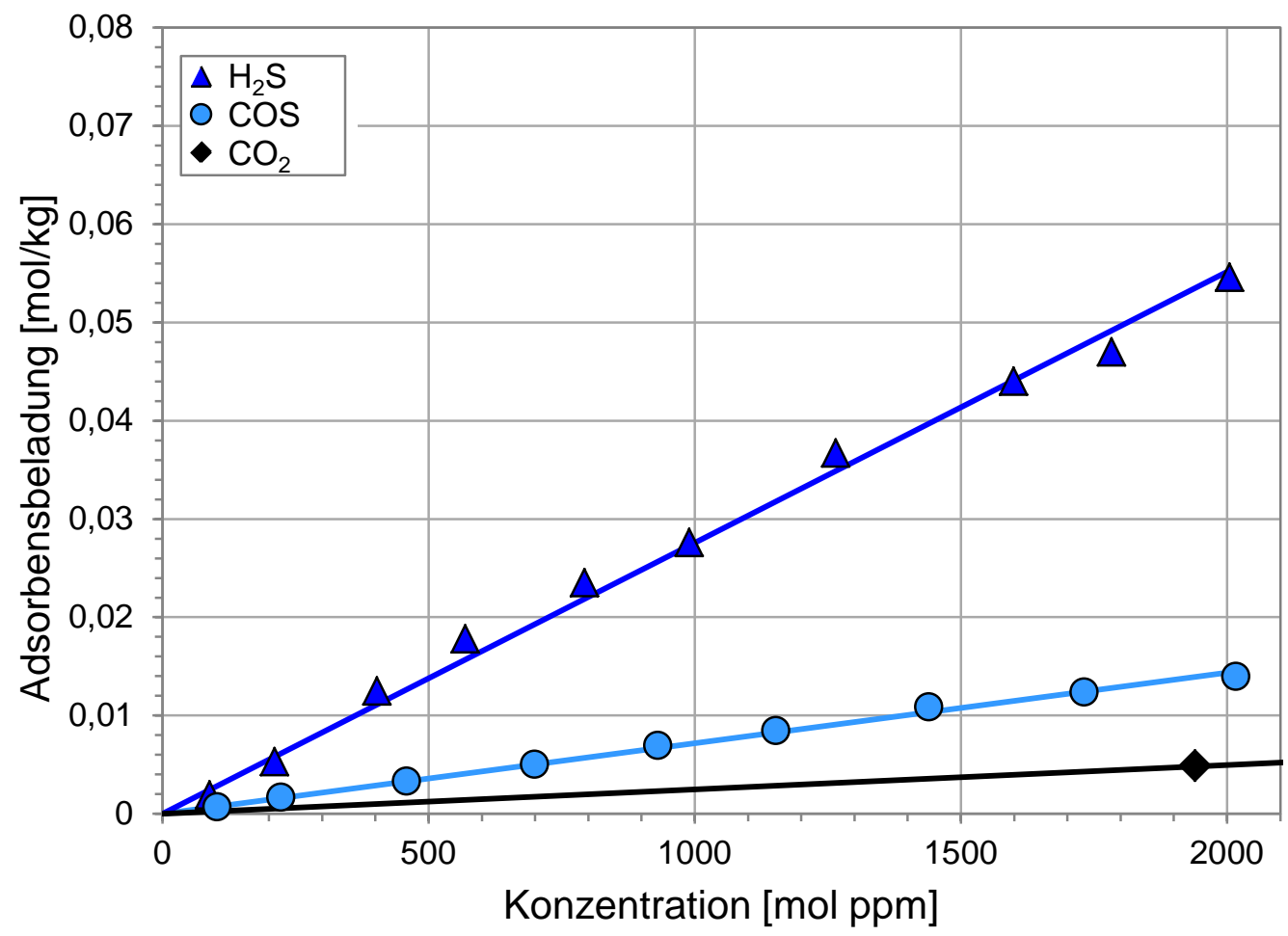

Abbildung 21: Isothermen für die Adsorption von $\mathrm{H}_{2} \mathrm{~S}$, $\mathrm{COS}$ und $\mathrm{CO}_{2}$ aus $\mathrm{CH}_{4}$ am Silica-Alumina-gel

Das lediglich leicht polarere $\mathrm{H}_{2} \mathrm{~S}$ weist gegenüber COS ein 4-fach stärkeres Adsorptionsverhalten auf. Gegenüber dem unpolaren $\mathrm{CO}_{2}$ im gezeigten Konzentrationsbereich gar ein um den Faktor 11 stärkeres Verhalten. Das Adsorptionsverhalten dieser schwach adsorbierenden Verbindungen ließ sich mit der Henry-Gleichung beschreiben.

Tabelle 2 gibt eine Auflistung der angepassten Isothermenparameter sowie der Anpassungsgüte ausgedrückt durch das Bestimmtheitsmaß $R^{2}$.

Tabelle 13: Angepasste Isothermenparameter binärer Stoffsysteme für das Silica-Alumina-Gel

\begin{tabular}{|r|c|c|c|c|}
\cline { 2 - 4 } \multicolumn{1}{|c|}{ Adsorptiv } & \multicolumn{1}{c|}{ Henry } & \multicolumn{2}{c|}{ Freundlich } & $\mathbf{R}^{2}$ \\
\hline $\mathbf{k}_{\mathbf{H}}$ & $\begin{array}{c}\mathbf{k}_{\mathbf{F}} \\
{[\mathbf{m o l} / \mathbf{m g}]}\end{array}$ & - & - & 0,99 \\
\hline $\mathbf{C O}_{2}$ & $2,48^{\star} 10^{-6}$ & - & - & 0,99 \\
\hline $\mathbf{C O S}$ & $7,18^{\star} 10^{-6}$ & - & - & 0,99 \\
\hline $\mathbf{H}_{2} \mathbf{S}$ & $2,76^{\star} 10^{-5}$ & $2,78^{\star} 10^{-3}$ & 0,689 & 0,98 \\
\hline $\mathbf{C H}_{3} \mathbf{S H}$ & - & 0,0172 & 0,517 & 0,99 \\
\hline $\mathbf{C}_{2} \mathbf{H}_{5} \mathbf{S H}$ & - & 0,0282 & 0,541 & 0,99 \\
\hline $\mathbf{C}_{3} \mathbf{H}_{7} \mathbf{S H}$ & - & 0,0136 & 0,679 & 0,99 \\
\hline $\mathbf{H}_{2} \mathbf{O}$ & - & & & \\
\hline
\end{tabular}




\section{Modifizierte Silica-Alumina-Gele}

Um einen möglichen Einfluss des Aluminiumgehaltes des Silica-Alumina-GelStandardmaterials auf die Schwefelwasserstoffadsorption beschreiben zu können, wurden zwei zu diesem Zweck modifizierte Variationen mit verändertem Aluminiumgehalt untersucht. Durch Charakterisierung mittels Stickstoff konnte gezeigt werden, dass sich die strukturellen Eigenschaften dieser Materialien dem Standardmaterial entsprechen (vgl. Abschnitt 3.1.1). Möchte man ternäre Stoffsysteme, also Stoffsysteme mit zwei adsorbierbaren Verbindungen in einem Matrixgas, beschreiben, benötigt man als Grundlage die Adsorptionsisothermen der binären Stoffsysteme der beteiligten Spezies. Im Hinblick auf eine genauere Betrachtung des ternären Stoffsystems mit den Adsorptiven $\mathrm{H}_{2} \mathrm{~S}$ und $\mathrm{CO}_{2}$ in der Matrix $\mathrm{CH}_{4}$ wurden dementsprechend zunächst die binären Systeme $\left(\mathrm{H}_{2} \mathrm{~S}\right.$ in $\mathrm{CH} 4, \mathrm{CO}_{2}$ in $\left.\mathrm{CH}_{4}\right)$ für die Silica-AluminaGel-Variationen vermessen. Abbildung 22 und Abbildung 23 zeigen die Isothermen für diese Stoffsysteme im Vergleich zu den Messungen für das Silica-Alumina-Gel-Standardmaterial.

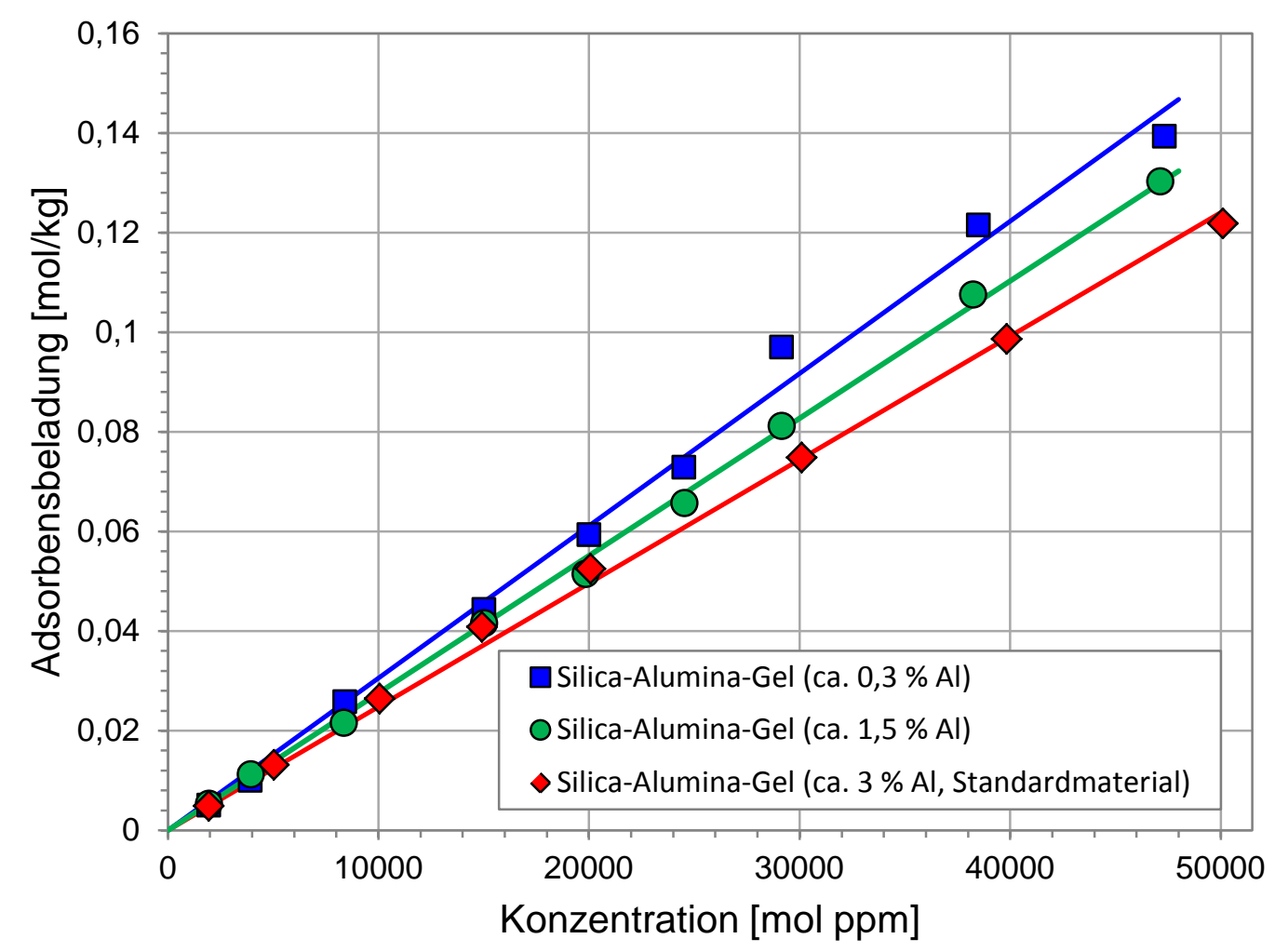

Abbildung 22: $\mathrm{CO}_{2}$-Adsorptionsisothermen für die verschiedenen Silica-Alumina-Gel-Varianten

Abbildung 22 lässt erkennen, dass die $\mathrm{CO}_{2}$-Adsorptionsisothermen für die drei SilicaAlumina-Gel-Varianten alle in der gleichen Größenordnung liegen. Alle drei Messreihen lassen sich in sehr guter Näherung durch die Henry-Gleichung beschreiben. Die ermittelten 
Beladungen lassen sich mit der leicht steigenden BET-Oberfläche bei gleichzeitig abnehmenden Aluminiumgehalten (vgl. Tabelle 4) korrelieren.

Ein unterschiedliches Bild zeigt sich bei Betrachtung der in Abbildung 23 dargestellten $\mathrm{H}_{2} \mathrm{~S}$ Isothermen für diese Adsorbentien.

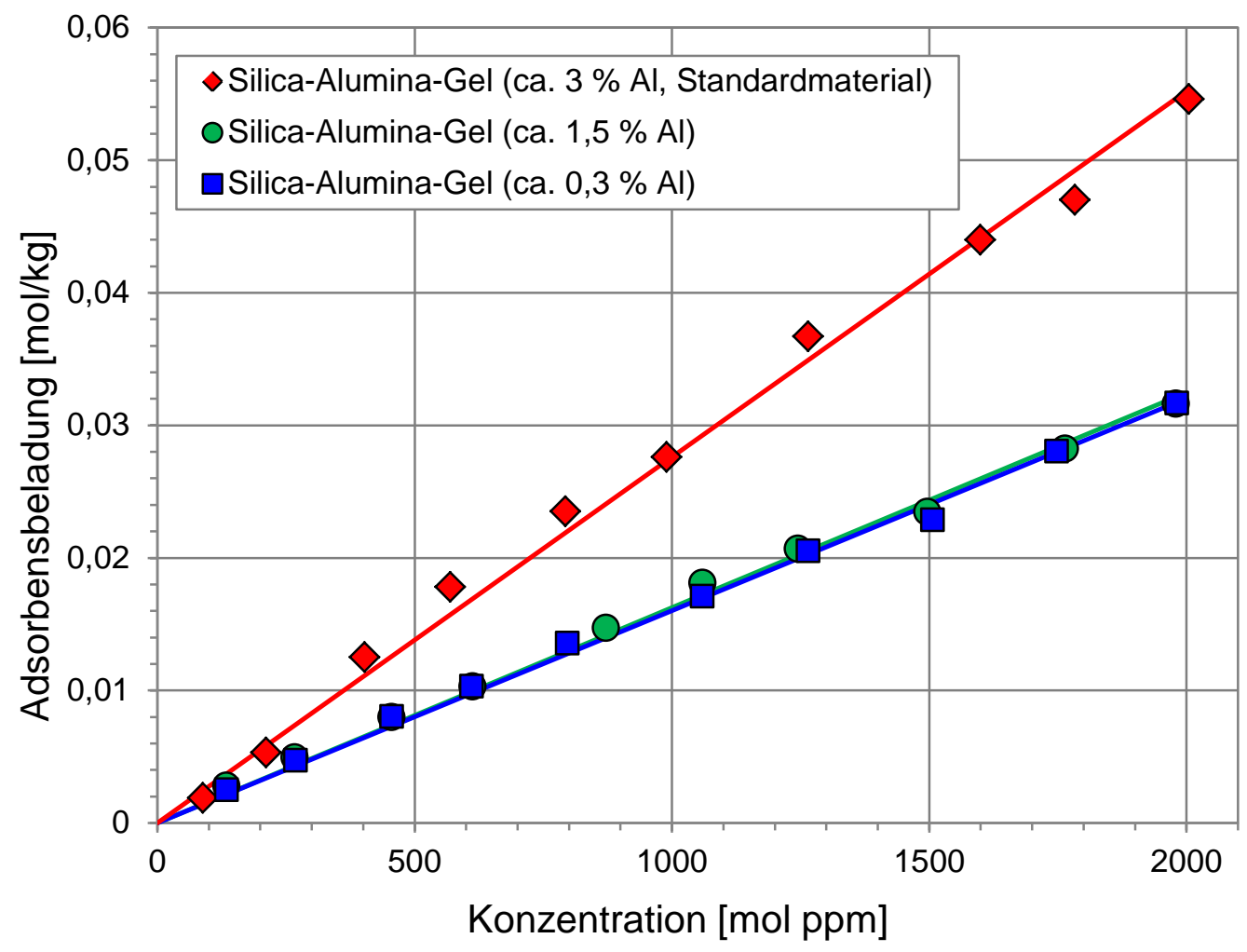

Abbildung 23: $\mathrm{H}_{2} \mathrm{~S}$-Adsorptionsisothermen für die verschiedenen Silica-Alumina-Gel-Varianten

Zwar zeigen wiederum alle Messreihen ein Henry-Verhalten, jedoch weisen die Modifikationen im Vergleich zum Standardmaterial deutlich geringere Beladungskapazitäten für Schwefelwasserstoff auf. Weiterhin weisen die Modifikationen trotz unterschiedlichem Aluminiumgehalt und dementsprechend unterschiedlicher BET-Oberfläche sehr ähnliche Beladungskapazitäten auf. Tabelle 14 gibt eine Auflistung der angepassten HenryIsothermenparameter und der entsprechenden Bestimmtheitsmaße.

Tabelle 14: Angepasste Isothermenparameter der binären Stoffsysteme $\left(\mathrm{H}_{2} \mathrm{~S}\right.$ in $\mathrm{CH}_{4}$ und $\mathrm{CO}_{2}$ in $\left.\mathrm{CH}_{4}\right)$ für alle Silica-Alumina-Gel-Varianten

\begin{tabular}{|l|c|c|c|}
\hline \multicolumn{1}{|c|}{ Adsorbens } & Adsorptiv & $\begin{array}{c}\mathbf{k}_{\mathbf{H}} \\
{[\mathbf{m o l} / \mathbf{m g}]}\end{array}$ & $\mathbf{R 2}$ \\
\hline \multirow{2}{*}{$\begin{array}{l}\text { Silica-Alumina-Gel } \\
\text { (ca. 1,5 \% Al) }\end{array}$} & $\mathbf{C O}_{2}$ & $2,76^{\star} 10^{-6}$ & 0,99 \\
\hline \multirow{2}{*}{$\begin{array}{l}\text { Silica-Alumina-Gel } \\
\text { (ca. 0,3 \% Al) }\end{array}$} & $\mathbf{H}_{2} \mathbf{S}$ & $1,62^{\star} 10^{-5}$ & 0,99 \\
\cline { 2 - 4 } & $\mathbf{C O}_{2}$ & $3,08^{\star} 10^{-6}$ & 0,99 \\
\hline
\end{tabular}




\subsubsection{Adsorptionsisothermen an 5A Zeolith}

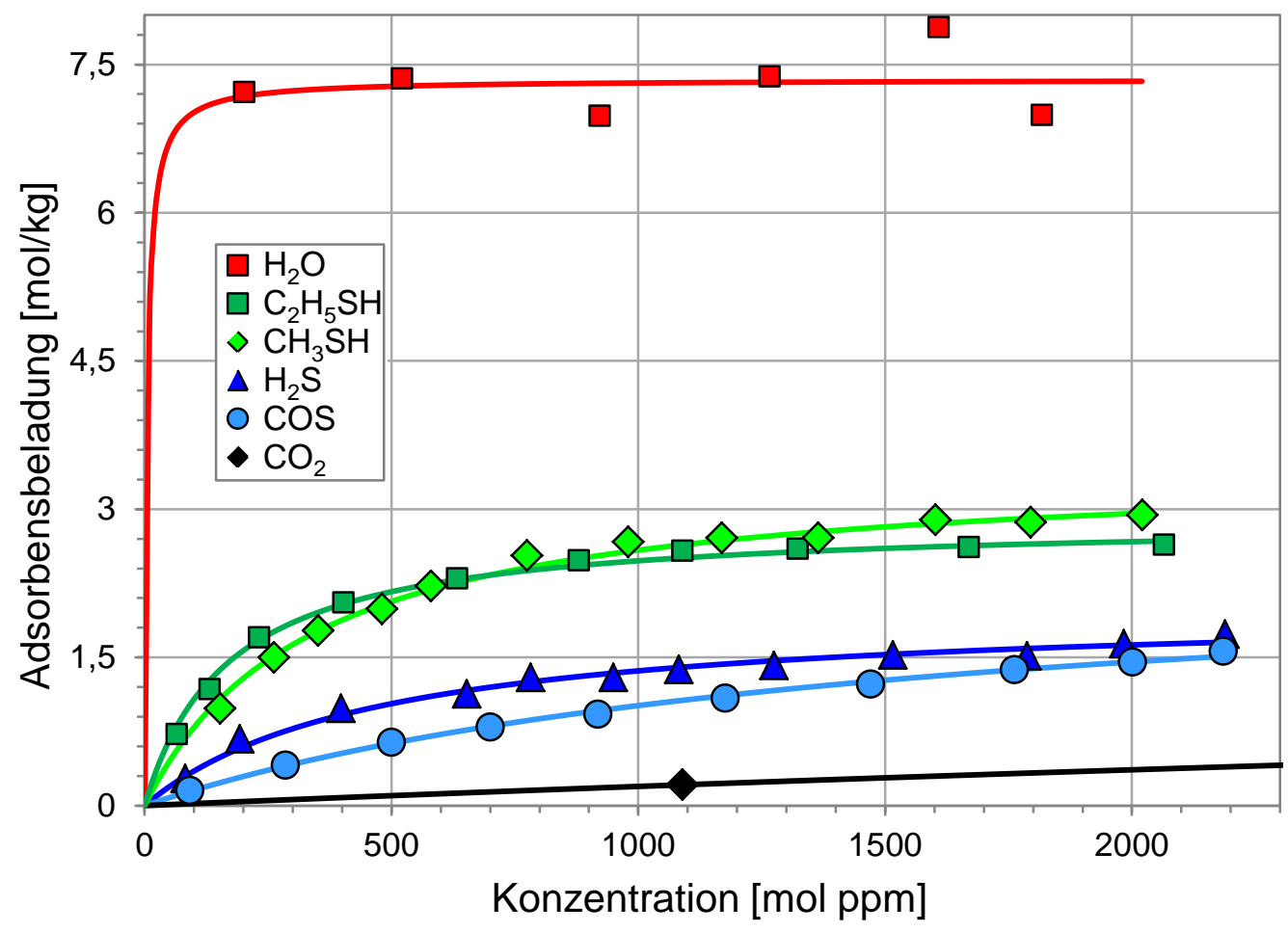

Abbildung 24: Isothermen für die Adsorption verschiedener Schwefelverbindungen, $\mathrm{CO}_{2}$ und $\mathrm{H}_{2} \mathrm{O}$ aus $\mathrm{CH}_{4}$ am Zeolith des Typs $5 \mathrm{~A}$

Abbildung 24 zeigt die Isothermen der Adsorption am Zeolithen des Typs 5A. Auf Messungen mit Propylmercaptan wurde aufgrund der sehr langen Zeit zur Einstellung des Gleichgewichts verzichtet.

$\mathrm{H}_{2} \mathrm{O}$ zeigte im Langmuir-Plateau Beladungen im Bereich $7.35 \mathrm{~mol} / \mathrm{kg}$. Maximale erreichte Beladungen für die untersuchten Mercaptane liegen im Bereich 2,4-2,9 mol/kg; für $\mathrm{H}_{2} \mathrm{~S}$ und COS im Bereich 1,5-1,6 mol/kg. Für $\mathrm{CO}_{2}$, dessen Adsorption bis $5 \mathrm{~mol} \%$ untersucht wurde, zeigt der $5 \mathrm{~A}$ Zeolith im dargestellten Konzentrationsbereich die geringsten Kapazitäten (ca. $0,4 \mathrm{~mol} / \mathrm{kg}$ ). Das Adsorptionsverhalten der betrachteten Stoffsysteme ließ sich durchweg mit der Langmuir-Gleichung beschreiben. Tabelle 15 zeigt die Isothermenparameter und die Güte der Anpassung ausgedrückt durch das Bestimmtheitsmaß $\mathrm{R}^{2}$. 
Tabelle 15: Parameter der Isothermenanpassungen für binäre Stoffsysteme an 5A Zeolith

\begin{tabular}{|r|c|c|c|}
\hline Adsorptiv & $\begin{array}{c}\mathbf{X}_{\text {Mon }} \\
{[\mathrm{mol} / \mathbf{k g}]}\end{array}$ & $\begin{array}{c}\mathbf{b} \\
{[-]}\end{array}$ & $\mathbf{R}^{2}$ \\
\hline $\mathbf{C O}_{2}$ & 2,724 & $7,794^{\star} 10^{-5}$ & 0,99 \\
\hline $\mathbf{C O S}$ & 2,553 & $6,62^{\star} 10^{-4}$ & 0,99 \\
\hline $\mathbf{H}_{2} \mathbf{S}$ & 2,013 & $2,1^{\star} 10^{-3}$ & 0,98 \\
\hline $\mathrm{CH}_{3} \mathbf{S H}$ & 3,451 & $2,98^{\star} 10^{-3}$ & 0,99 \\
\hline $\mathbf{C}_{2} \mathbf{H}_{5} \mathbf{S H}$ & 2,90 & $5,91^{\star} 10^{-3}$ & 0,99 \\
\hline $\mathbf{H}_{2} \mathbf{O}$ & 7,347 & 0,2111 & 0,98 \\
\hline
\end{tabular}

\subsection{Diskussion der binären Stoffsysteme}

\subsubsection{Adsorption an Silica-Alumina-Gel}

Die untersuchten Stoffsysteme lassen sich anhand des Adsorptionsverhaltens am mesoporösen Silica-Alumina-Gel in zwei Gruppen einteilen. Für das unpolare $\mathrm{CO}_{2}$ und die im Vergleich zu den Mercaptanen und $\mathrm{H}_{2} \mathrm{O}$ schwach polaren Schwefelverbindungen $\mathrm{H}_{2} \mathrm{~S}$ und COS (vgl. Tabelle 16) zeigen sich geringe Adsorptionskapazitäten, die sich im untersuchten Konzentrationsbereich sehr gut durch die Henry-Gleichung approximieren lassen. Für das am schwächsten adsorbierende Molekül $\mathrm{CO}_{2}$ wurde die Adsorption bis zu einer Feedkonzentration von $5 \mathrm{~mol} \%$ untersucht, um realistische Konzentrationen in Erdgasen nachzuempfinden. Selbst in diesem vergleichsweise hohen Konzentrationsbereich kann das Adsorptionsverhalten immer noch durch die Henry-Gleichung beschrieben werden. Das Adsorptionsverhalten der Mercaptane sowie des Wassers kann hingegen in sehr guter Näherung durch die Freundlich-Gleichung beschrieben werden. Betrachtet man die in Tabelle 16 aufgelisteten Dipolmomente der Adsorptivmoleküle als Maß für die Affinität des Moleküls zur polaren Adsorbensoberfläche des Silica-Alumina-Gels, lässt sich anhand der gefundenen Kapazitäten eine Reihenfolge aufstellen, nach der die Adsorptionskapazität am SilicaAlumina-Gel mit der Polarität des Adsorptivs zunimmt: $\mathrm{CO}_{2}<\mathrm{COS}<\mathrm{H}_{2} \mathrm{~S}<\mathrm{CH}_{3} \mathrm{SH}<$ $\mathrm{C}_{2} \mathrm{H}_{5} \mathrm{SH}<\mathrm{C}_{3} \mathrm{H}_{7} \mathrm{SH}<\mathrm{H}_{2} \mathrm{O}$. Bei dieser Betrachtung sind jedoch mögliche Wechselwirkungen auf Grund höherer Momente nicht einbezogen. 
Tabelle 16: Dipolmomente der Adsorptivmoleküle $[68,69,70]$

\begin{tabular}{|l|c|}
\hline Adsorptiv & $\begin{array}{c}\text { Dipolmoment } \\
\text { [Debye] }\end{array}$ \\
\hline $\mathbf{H}_{2} \mathbf{O}$ & 1,84 \\
\hline $\mathbf{C}_{3} \mathbf{H}_{7} \mathbf{S H}$ & 1,60 \\
\hline $\mathbf{C}_{2} \mathbf{H}_{5} \mathbf{S H}$ & 1,58 \\
\hline $\mathbf{C H}_{3} \mathbf{S H}$ & 1,52 \\
\hline $\mathbf{H}_{2} \mathbf{S}$ & 0,98 \\
\hline $\mathbf{C O S}$ & 0,72 \\
\hline $\mathbf{C O}_{2}$ & 0 \\
\hline
\end{tabular}

Das beschriebene Verhalten zeigt sich besonders deutlich bei Betrachtung der homologen Reihe $\mathrm{H}_{2} \mathrm{~S}>\mathrm{CH}_{3} \mathrm{SH}>\mathrm{C}_{2} \mathrm{H}_{5} \mathrm{SH}>\mathrm{C}_{3} \mathrm{H}_{7} \mathrm{SH}$. Für die Mercaptane, die man formal gesehen aus Schwefelwasserstoff durch Ergänzung um eine, zwei bzw. drei Methylengruppen erhält, ergeben sich Kapazitätssteigerungen proportional zur Kettenlängenänderung.

\section{Modifizierte Silica-Alumina-Gele}

Bei den Untersuchungen des Stoffsystems $\mathrm{CO}_{2}$ in $\mathrm{CH}_{4}$ (Abbildung 22) zeigt sich ein Adsorptionsverhalten, dass sich mit den Ergebnissen der Adsorbenscharakterisierung mittels Stickstoffadsorption bei $77 \mathrm{~K}$ korrelieren lässt. Aus den BET-Oberflächen der drei Materialien lässt sich erkennen, dass die spezifische Oberfläche geringfügig steigt, je geringer der Aluminium-Gehalt ist. Dieser Trend lässt sich auch bei Betrachtung der vermessenen $\mathrm{CO}_{2}-$ Adsorptionsisothermen für diese verschiedenen Silica-Alumina-Gel-Varianten wiederfinden. Die Kapazitäten sind umso größer, je geringer der Aluminium-Gehalt und je höher die innere Oberfläche des Adsorbens ist.

Für die Interpretation der ermittelten (Abbildung 23) $\mathrm{H}_{2} \mathrm{~S}$-Isothermen sind zusätzliche Aspekte zu berücksichtigen, da es für das Adsorptiv $\mathrm{H}_{2} \mathrm{~S}$ prinzipiell zu zwei unterschiedlichen Bindungsmechanismen auf der Silica-Alumina-Gel-Oberfläche kommen kann.

\section{$\mathrm{H}_{2}$ S-Bindungsmechanismen an Silica-Alumina-Gel}

Die im Rahmen der Untersuchungen verwendeten Silica-Alumina-Gele weisen Aluminiumoxid-Anteile (angegeben als $\mathrm{Al}_{2} \mathrm{O}_{3}$ ) von $3 \%$ beim Standardmaterial und 1,5\% bzw. 0,3\% bei den modifizierten Materialien auf. Damit ist die Oberfläche dieser Adsorbentien heterogen. Gemäß den in Abbildung 25 dargestellten Schemata sollten zwei unterschiedliche Wechselwirkungsmechanismen mit der Adsorbensoberfläche möglich sein. 


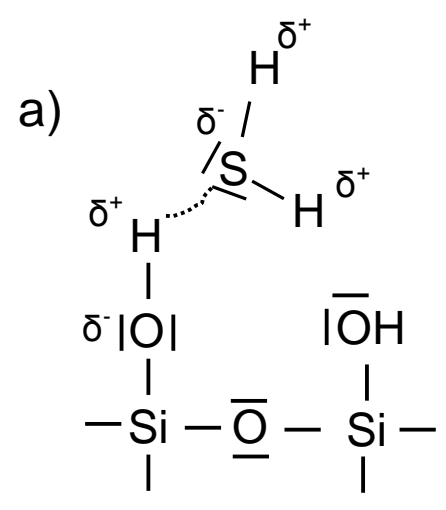

b)

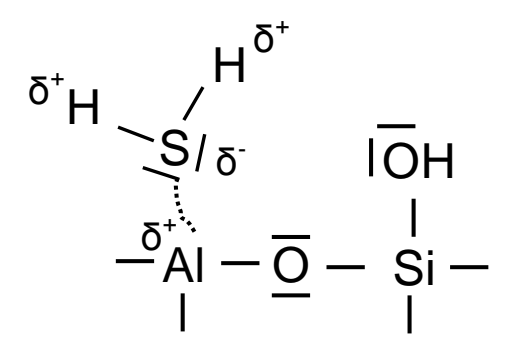

Abbildung 25: Bindungsmechanismen an der Silica-Alumina-Gel-Oberfläche. a) $\mathrm{H}_{2} \mathrm{~S}$-Bindung über Wasserstoffbrückenbindung; b) $\mathrm{H}_{2} \mathrm{~S}$-Bindung über Lewis-Säure-Base-Wechselwirkung

Der Schwefel bietet im $\mathrm{H}_{2} \mathrm{~S}$ eine negativ polarisierte Bindungsstelle. Zwischen dem Wasserstoff der Silanol-Gruppe an der Oberfläche des Silica-Alumina-Gels und dem Schwefel des $\mathrm{H}_{2} \mathrm{~S}$-Moleküls bilden sich Wasserstoffbrückenbindungen aus. Eine weitere Möglichkeit für $\mathrm{H}_{2} \mathrm{~S}$ zu adsorbieren, bietet das Aluminium, welches man sich als trivalentes Aluminiumion in tetraedrischer Koordination zu drei Sauerstoffatomen vorstellen muss [79, 80]. Aluminium kann als Lewis-Säure mit einem Elektronenpaar der Lewis-Base $\mathrm{H}_{2} \mathrm{~S}$ wechselwirken. Es kommt zu einer koordinativen Bindung. $\mathrm{CO}_{2}$, welches selber eine Lewis-Säure darstellt [81], geht keine Wechselwirkung mit dem Aluminium ein. Die beobachteten sehr geringen Beladungen sind auf schwächere Wechselwirkungen zurückzuführen (vgl. Abschnitt „Adsorption von Adsorptiven ohne Dipolmoment an polaren Adsorbentien“).

Ausgehend von der Hypothese, dass Schwefelwasserstoff bevorzugt Wechselwirkungen mit dem an der Oberfläche befindlichen Aluminium eingeht, konnte anhand der binären Untersuchungen zwar gezeigt werden, dass eine Verringerung des Aluminium-Gehaltes des SilicaAlumina-Gels auch eine Verminderung der $\mathrm{H}_{2} \mathrm{~S}-$ Kapazität zur Folge hat. Bei Betrachtung der in Abbildung 23 dargestellten $\mathrm{H}_{2} \mathrm{~S}$-Isothermen für die drei Silica-Alumina-Gel-Varianten zeigt sich jedoch ein unerwartetes Bild. Obwohl eine der beiden modifizierten Silica-Alumina-GelVarianten (ca. 1,5\% Al) einen deutlich höheren Aluminium-Gehalt aufweist, sind die erreichten Kapazitäten im Vergleich zur anderen Silica-Alumina-Gel-Variante (ca. 0,3\% Al) nur unwesentlich größer.

Diesen Unterschied würde man deutlich ausgeprägter erwarten. Wenn sich das gesamte Aluminium an der Oberfläche des Silica-Alumina-Gels befindet und daher für die adsorbierenden $\mathrm{H}_{2} \mathrm{~S}$-Moleküle erreichbar ist, würde man erwarten, dass die entsprechende Isotherme ungefähr in der Mitte zwischen den beiden anderen Isothermen liegt. Eine weiterführende 
Betrachtung dieser Zusammenhänge findet sich in Abschnitt 4.4.1, in welchem die Ergebnisse der ternären Stoffsysteme $\left(\mathrm{H}_{2} \mathrm{~S}+\mathrm{CO}_{2}\right.$ in $\left.\mathrm{CH}_{4}\right)$ mit in die Diskussion einfließen.

Die Reihenfolge, nach der eine Verbindung besser adsorbiert, je polarer sie ist, gilt ebenfalls für die modifizierten Silica-Alumina-Gele.

\subsubsection{Adsorption an 5A Zeolith}

Alle untersuchten Adsorptive für den Zeolithen des Typs 5A zeigen ein Adsorptionsverhalten, dass sich in sehr guter Näherung durch die Langmuir-Gleichung beschreiben lässt. Auch für dieses Adsorbens wurde die Adsorption von $\mathrm{CO}_{2}$ bis $5 \mathrm{~mol} \%$ untersucht. Prinzipiell zeigt sich für die untersuchten Stoffsysteme, dass sich die Adsorptionskapazitäten mit der Polarität der Adsorptive erhöhen. Einer differenzierteren Betrachtung innerhalb dieser Argumentation muss die Isotherme für Ethylmercaptan $\left(\mathrm{C}_{2} \mathrm{H}_{5} \mathrm{SH}\right)$ unterzogen werden. Die vermessenen Mercaptan-Isothermen sind in Abbildung 26 im Detail dargestellt.

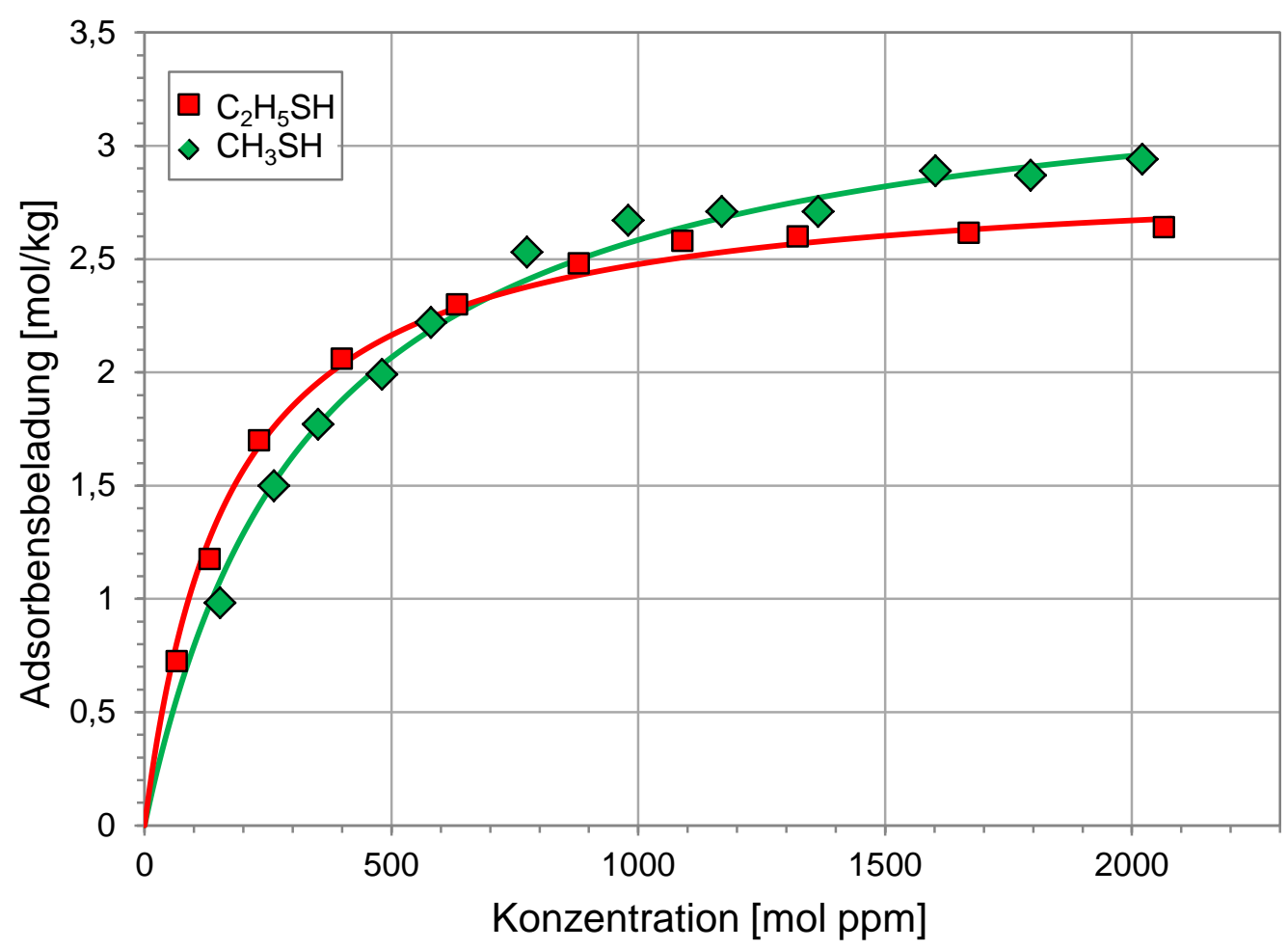

Abbildung 26: Isothermen für die Adsorption von Methyl- und Ethylmercaptan aus $\mathrm{CH}_{4}$ am Zeolith $5 \mathrm{~A}$

Im Bereich kleiner Konzentrationen ( $<500 \mathrm{~mol}$ ppm) wird das erwartete Verhalten beobachtet. Das im Vergleich zu Methylmercaptan leicht polarere Ethylmercaptan (vgl. Tabelle 16) erreicht in diesem Bereich höhere Adsorptionskapazitäten. Dies lässt sich auch bei Betrach- 
tung des Langmuir-Parameters $b$ feststellen. Je größer die Affinität des Adsorptivs zum Adsorbens, desto größer ist b (vgl. Tabelle 15) und demzufolge die Steigung der Isotherme in Ursprungsnähe. Betrachtet man die Verläufe der beiden Mercaptan-Isothermen $\left(\mathrm{CH}_{3} \mathrm{SH}\right.$, $\mathrm{C}_{2} \mathrm{H}_{5} \mathrm{SH}$ ) über den gesamten Untersuchungsbereich, zeigt sich bei einer Konzentration im Bereich $700 \mathrm{~mol}$ ppm ein Schnittpunkt beider Isothermen. Die Langmuir-Isotherme für das polarere Ethylmercaptan zeigt im Untersuchungsbereich ein niedrigeres Plateau als die Methylmercaptan-Isotherme. Hierfür bieten sich zwei konsistente Erklärungen an.

Bei der Herstellung des im Rahmen dieser Untersuchungen verwendeten Zeolithen des Typs $5 \mathrm{~A}$ werden $\mathrm{Na}^{+}$-Ionen durch $\mathrm{Ca}^{2+}$-Ionen ausgetauscht. Kast gibt für Ca-Käfige eine effektive Porenöffnung von 5,0 $\AA$ an [38]. Da bei der Synthese kein vollständiger Austausch erreicht wird (> 65\% lauf Herstellerdatenblatt) (vgl. Tabelle 3), wird der Zeolith noch Na-Käfige aufweisen (effektive Porenöffnung 4,2 $\AA$ [38]). Geht man von einem einfachen Größenausschluss aus, sind Ethylmercaptan-Moleküle (kritischer Durchmesser 5,1 $\AA$ [71]) nicht in der Lage, in die Na-Käfige zu diffundieren. Für den kritischen Durchmesser des Methylmercaptan lassen sich Werte im Bereich von 3,8-4,5 $\AA$ in der Literatur finden $[71,72]$. Damit sollten Na-Käfige für Methylmercaptan zugänglich sein, und es ergibt sich eine geringere Anzahl erreichbarer Adsorptionsplätze für Ethylmercaptan.

Außerdem verringert sich vom Methylmercaptan zum Ethylmercaptan die molare Kapazität im Bereich der Sättigung, weil der Platzbedarf des Ethylmercaptans im Porensystem infolge der längeren Molekülkette höher ist.

Für die Adsorption von $\mathrm{H}_{2} \mathrm{~S}$ an Zeolithen ließen sich in der Literatur einige Beladungskapazitäten finden. Tabelle 17 fasst einige Ergebnisse verschiedener Autoren zusammen.

Tabelle 17: $\mathrm{H}_{2} \mathrm{~S}$-Adsorptionskapazitäten an Zeolithen im Bereich von $2000 \mathrm{~mol}$ ppm (Werte umgerechnet)

\begin{tabular}{|l|l|l|l|}
\hline Versuchsgas & $\begin{array}{l}\text { Temperatur } \\
{\left[{ }^{\circ} \mathbf{C}\right]}\end{array}$ & $\begin{array}{l}\text { Adsorbensbeladung } \\
{[\mathrm{mol} / \mathbf{k g}]}\end{array}$ & Zitat \\
\hline Reinstoff & 20 & $\approx 1,9$ & $(12)$ \\
\hline Reinstoff & 20 & $\approx 0,35-1,03$ & $(18)$ \\
\hline Reinstoff & 25 & $\approx 1,47-2,05$ & $(10)$ \\
\hline Reinstoff & 25 & $\approx 1,99$ & $(4)$ \\
\hline Gasmischung $\left(\mathrm{H}_{2} \mathrm{~S}+\mathrm{CH}_{4}\right)$ & 25 & 1,64 & Eigener Messwert \\
\hline
\end{tabular}

Die Werte sind auf Grund unterschiedlicher Untersuchungsmethoden und Materialien nur bedingt vergleichbar. Dennoch wurden $\mathrm{H}_{2} \mathrm{~S}$-Kapazitäten in der gleichen Größenordnung gefunden. Verglichen mit Kapazitäten für die Adsorption von reinem $\mathrm{H}_{2} \mathrm{~S}$ ist die für die $\mathrm{Ad}$ sorption von $\mathrm{H}_{2} \mathrm{~S}$ aus reinem Methanstrom bestimmte Kapazität etwas geringer. 


\subsubsection{Vergleich der Adsorbentien}

\section{Einfluss des Trägergases auf die Adsorption}

Abbildung 27 vergleicht die Wasseradsorption an beiden Adsorbentien. Zur Einordnung der Kapazität sind schematisch die Isothermen der in den Abbildung 20 Abbildung 24 gezeigten Stoffsysteme mit dargestellt.

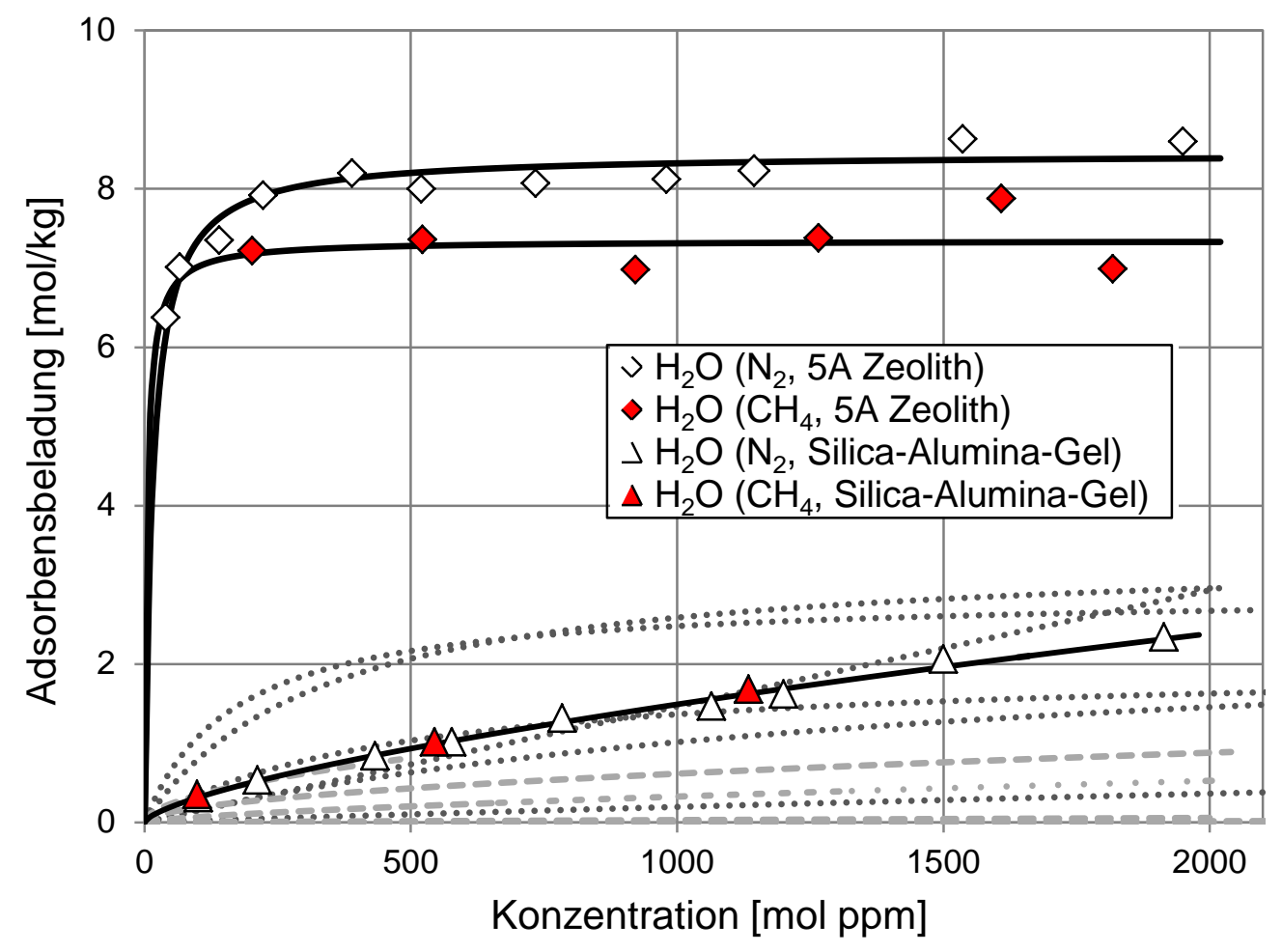

Abbildung 27: Isothermen für die Adsorption von $\mathrm{H}_{2} \mathrm{O}$ aus $\mathrm{CH}_{4}$ und $\mathrm{N}_{2}$ am Zeolith $5 \mathrm{~A}$ und am SilicaAlumina-Gel

Erwartungsgemäß zeigen sich für Wasser die größten Adsorptionskapazitäten. Auf Grund seiner starken Polarität (vgl. Tabelle 16) besteht zwischen den Wassermolekülen und den polaren Oberflächen beider Adsorbentien die größte Affinität. Ein Einfluss des Trägergases auf die $\mathrm{H}_{2} \mathrm{O}$-Kapazität des mesoporösen Silica-Alumina-Gels konnte ausgeschlossen werden. Für Methan (99,95 \%) und Stickstoff (99,99 \%) konnten vergleichbare Kapazitäten ermittelt werden. Ein gegenteiliger Effekt zeigt sich für die $\mathrm{H}_{2} \mathrm{O}$-Adsorption am mikroporösen Zeolithen. Bei Stickstoff als Trägergas kommt es im Vergleich zu Methan zu deutlich höheren Adsorptionskapazitäten. Dieser Unterschied lässt sich auf zwei Effekte zurückführen. Da vor jedem Versuch die Schüttung mit dem jeweiligen Trägergas abgesättigt wird, kann es auf Grund von Spurenfeuchte und weiteren Verunreinigungen im Spurenbereich der Trägergase 
zu unterschiedlich großen Vorbeladungen des Zeolithen kommen. Weiterhin sind beide Trägergasmoleküle sterisch in der Lage, in den Poren des Zeolithen zu adsorbieren $\left(d_{\text {krit, }}\right.$ $\mathrm{CH}_{4}=4,0 \AA, \mathrm{d}_{\text {krit, }} \mathrm{N}_{2}=3,0 \AA$ [38]). Das geringere Plateau der Wasserisotherme bei Methan als Trägergas im Vergleich zu Stickstoff als Trägergas ist vermutlich auf ein Zusammenwirken beider Effekte zurückzuführen. Adsorbierendes Wasser ist offenbar trotz seiner sehr hohen Polarität nicht in der Lage, alle Moleküle, die sich im Mikroporensystem des Zeolithen befinden, zu verdrängen. Ein Teil der zur Verfügung stehenden Adsorptionsplätze bleibt von anderen Molekülen besetzt.

Vergleicht man alle für beide Adsorbentien vermessenen Stoffsysteme, zeigt sich ein erwartetes Bild. Am Zeolith wurden für alle Adsorptive im untersuchten Konzentrationsbereich durchweg deutlich höhere Adsorptionskapazitäten erreicht. Silica-Alumina-Gele erreichen höhere Kapazitäten in vielen Fällen erst bei höheren Konzentrationen [32]. Mit den diskutierten Einschränkungen für das Ethylmercaptan lässt sich jedoch für beide Materialien die gleiche Reihenfolge für die Adsorbierbarkeit der untersuchten Moleküle beobachten. Für beide Adsorbentien gilt: Je größer die Polarität und damit die Affinität des Adsorptivs zur polaren Adsorbensoberfläche, desto größer ist die Adsorptionskapazität.

Diese gefundene Reihenfolge der Adsorbensbeladungen ist vergleichbar mit einer Reihenfolge, die Maddox [21] angibt. Er korreliert ebenfalls die steigende Adsorptivpolarität mit höheren Kapazitäten, gibt jedoch das leicht polare Carbonylsulfid als am schwächsten adsorbierende Verbindung an. Dies steht im Widerspruch zur Einordnung der Kapazitäten nach den Adsorptivpolaritäten. Demnach stellt das unpolare $\mathrm{CO}_{2}$, wie im Rahmen dieser Arbeit nachgewiesen, das schwächste Adsorptiv dar. Für $\mathrm{H}_{2} \mathrm{~S}$, leichte Mercaptane und Wasser sind die Reihenfolgen wiederum kongruent. Die experimentell belegte Reihenfolge von Groninger [20] untermauert im relevanten, geringen Konzentrationsbereich die hier gefundene Reihenfolge. Er gibt in seinem Beitrag die Reihenfolge $\mathrm{CO}_{2}<\mathrm{COS}<\mathrm{H}_{2} \mathrm{~S}<\mathrm{CH}_{3} \mathrm{SH}$ an.

\section{Einfluss der Polarität des Adsorptivs}

\section{Adsorption von Adsorptiven mit Dipolmomenten an polaren Adsorbentien}

Für die Beschreibung der Adsorbierbarkeit einer Verbindung lassen sich in den relevanten Monographien [47, 38, 35] grundlegende Selektivitätseinteilungen finden. 
Diese Prinzipien eignen sich als Faustregel zur Vorhersage einfacher Tendenzen. Die Reihenfolge der Adsorbierbarkeit für die im Rahmen dieser Arbeit betrachteten Adsorptive lässt sich damit jedoch nicht konsistent beschreiben. Carbonylsulfid müsste gemäß dem statischen Kapazitätseffekt auf Grund des geringeren Dampfdrucks auf beiden untersuchten Adsorbentien besser adsorbieren als Schwefelwasserstoff. In den untersuchten Systemen ist der Gleichgewichtseffekt ausschlaggebend, der sich direkt mit Hilfe der Stärke der Wechselwirkung zwischen Adsorptiv und der polaren Adsorbensoberfläche diskutieren lässt. Ein Maß dafür sind Unterschiede in der Polarität der Adsorptive (vgl. Abschnitt 2.3).

Wie in Abschnitt 2.3 gezeigt wurde, ist das molekulare Dipolmoment zur Beschreibung von Wechselwirkungen zwischen Adsorbens und Adsorptiv wichtig. Es lässt eine Korrelation mit einer experimentell gut zugänglichen Größe wie der Adsorbensbeladung zu. Es wurde jedoch darauf hingewiesen, dass das Dipolmoment nicht alleine ausschlaggebend für eine Bewertung eines Adsorptionsprozesses sein kann. Die lokale Verteilung der Elektronendichte innerhalb eines Moleküls ist bestimmend für die Wechselwirkung zwischen Adsorbens und Adsorptiv.

Um entsprechend Kapitel 2.4 Aussagen über die Rolle des Schwefelatoms innerhalb der untersuchten Moleküle auf die Wechselwirkung mit dem Adsorbens zu erhalten, wurden Partialladungen der Atome in den Molekülen berechnet. Diese Zusammenhänge sollen im Folgenden am Beispiel der Stoffgruppe der Mercaptane diskutiert werden.

Die aus dem molekularen, elektrostatischen Potenzial (vgl. Abschnitt 2.4) berechneten Partialladungen zeigen, dass das Schwefelatom im Falle der Mercaptane eine negative Partialladung aufweist. Tabelle 18 gibt eine Auflistung der Partialladung des Schwefelatoms innerhalb der verschiedenen Moleküle.

Tabelle 18: Berechnete Partialladungen des Schwefelatoms für die untersuchten Mercaptane

\begin{tabular}{|l|c|c|}
\hline Thiole & $\begin{array}{c}\text { Partialladung auf } \\
\text { dem S-Atom }\end{array}$ & $\begin{array}{c}\text { Dipolmoment } \\
\text { [Debye] }\end{array}$ \\
\hline $\mathbf{H}_{2} \mathbf{S}$ & $-0,335$ & $\mathbf{0 , 9 8}$ \\
\hline $\mathbf{C H}_{3} \mathbf{S H}$ & $-0,324$ & $\mathbf{1 , 5 2}$ \\
\hline $\mathbf{C}_{2} \mathbf{H}_{5} \mathbf{S H}$ & $-0,350$ & $\mathbf{1 , 5 8}$ \\
\hline $\mathbf{C}_{3} \mathbf{H}_{7} \mathbf{S H}$ & $-0,352$ & $\mathbf{1 , 6 0}$ \\
\hline
\end{tabular}

Bezieht man die Dipolmomente der betrachteten Stoffgruppe mit in die Argumentation ein, lässt sich eine Korrelation mit den berechneten Partialladungen erkennen. Bis zur Kettenlänge C3 steigen die Partialladungen des funktionellen Atoms (Schwefel) an. Dabei ist auch ein ansteigender Trend in den Dipolmomenten zu beobachten. Der Trend lässt sich auch für die experimentell bestimmten Mercaptan-Kapazitäten beobachten. Je höher das Dipolmoment der Verbindung und entsprechend auch die negative Partialladung des Schwefels, desto 
höher sind die ermittelten Kapazitäten. Dies zeigt, dass das Schwefelatom für Moleküle der homologen Reihe $\mathrm{H}_{2} \mathrm{~S}>\mathrm{CH}_{3} \mathrm{SH}>\mathrm{C}_{2} \mathrm{H}_{5} \mathrm{SH}>\mathrm{C}_{3} \mathrm{H}_{7} \mathrm{SH}$ für die Wechselwirkung mit der Adsorbensoberfläche eine wichtige Rolle spielt.

\section{Adsorption eines Adsorptivs ohne Dipolmoment an polaren Adsorbentien}

Für das Adsorptiv $\mathrm{CO}_{2}$ wurde die Adsorption bis zu einer Konzentration von 5 mol \% betrachtet, da es in realen Erdgasen häufig in weitaus höheren Konzentrationen als Schwefelverbindungen enthalten ist. Die Konzentrationen der sauren Verbindungen können jedoch je nach Förderstätte stark variieren [3]. Abbildung 28 zeigt den Vergleich der entsprechenden Isothermen für das Silica-Alumina-Gel und den 5A Zeolith.

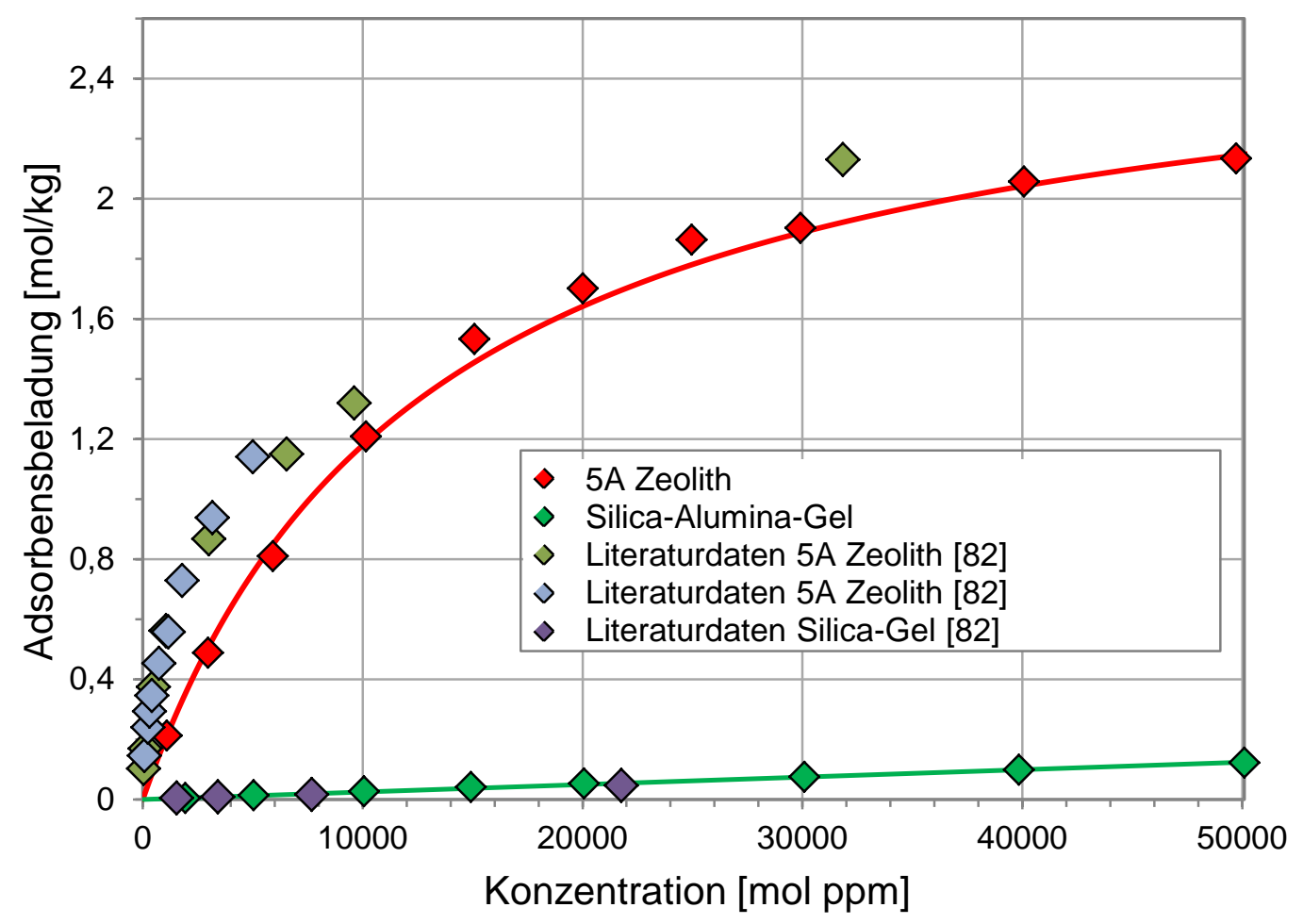

Abbildung 28: Isothermen für die Adsorption von $\mathrm{CO}_{2}$ aus Methan am Silica-Alumina-Gel und $5 \mathrm{~A}$ Zeolith

Die Abbildung verdeutlicht, die großen Unterschiede zwischen den beiden Adsorbentien in den erreichten $\mathrm{CO}_{2}$-Kapazitäten. Demnach weist das Silica-Alumina-Gel am rechten Rand des Untersuchungsbereichs (im Bereich von $5 \mathrm{~mol} \% \mathrm{CO}_{2}$ ) lediglich ca. $6 \%$ der Kapazität des Zeolithen auf. Eine Gegenüberstellung mit aktuellen Literaturwerten [82] für die Adsorption von $\mathrm{CO}_{2}$ an einem Silica-Gel und einem $5 \mathrm{~A}$ Zeolith eines anderen Herstellers (Hersteller: Grace) zeigt eine gute Vergleichbarkeit. Die dargestellten Werte wurden in statischen volu- 
metrischen oder gravimetrischen Versuchsapparaturen vermessen. Die ermittelten $\mathrm{CO}_{2}$ Beladungen für das Silica-Alumina-Gel lassen sich sehr gut mit den hier bestimmten Kapazitäten des verwendeten Silica-Alumina-Gels vergleichen. Der Kapazitätsvergleich der $5 \mathrm{~A}$ Zeolithe zeigt eine geringe Abweichung zwischen Messwerten und Literaturwerten. Die Beladungswerte und auch die Verläufe liegen jedoch in der gleichen Größenordnung.

Dennoch ist auffällig, dass es für den Zeolithen im Vergleich zu den stärker adsorbierenden Schwefelverbindungen selbst in diesem vergleichsweise hohen Konzentrationsbereich noch zu keinem Beladungsplateau kommt. Sogar das Adsorptionsverhalten des $\mathrm{CO}_{2}$ auf dem Silica-Alumina-Gel kann über diesen großen Untersuchungsbereich weiterhin mit sehr guter Näherung durch die Henry-Gleichung beschrieben werden. Dies spiegelt die geringe Affinität des unpolaren $\mathrm{CO}_{2}$ zu beiden Adsorbensoberflächen wider. Beispielsweise kann das gering polare Adsorptiv Schwefelwasserstoff auf beiden Adsorbentien vergleichbare Kapazitäten bereits im Spurenbereich (2000 mol ppm) erreichen. Trotzdem haben die für die Bindung des $\mathrm{CO}_{2}$ auf dem Zeolith vorherrschenden elektrostatischen Wechselwirkungsmechanismen wie z. B. Ion-Multipol-Wechselwirkungen offenbar einen deutlich stärkeren Einfluss. $\mathrm{CO}_{2}$ besitzt ein elektrisches Quadrupolmoment, welches im stark polaren Porensystem des Zeolithen zu Wechselwirkungen führen kann. Im Vergleich dazu scheinen die sehr geringen Kapazitäten auf dem Silica-Alumina-Gel lediglich auf schwächere Wechselwirkungen wie van der WaalsKräfte zurückzuführen zu sein. Der bei Adsorptiven mit Dipolmoment wichtige Bindungsmechanismus über Wasserstoffbrückenbindungen zur Silica-Oberfläche spielt bei der Adsorption des unpolaren $\mathrm{CO}_{2}$ vermutlich keine Rolle. Ebenso ist nicht anzunehmen, das $\mathrm{CO}_{2}$ an Aluminium bindet, da beides Lewis-Säuren sind. 


\subsection{Ternäre Stoffsysteme}

\subsection{1 Überblick}

Bei den meisten Gasaufbereitungsprozessen liegen Gasgemische mit mehreren adsorbierbaren Verbindungen vor. Dementsprechend kommt es bei der Entschwefelung z. B. von Erdgas immer auch zu Mehr- oder Multikomponentenadsorption. Von besonderem Interesse in diesem Zusammenhang sind $\mathrm{CO}_{2}$ und $\mathrm{H}_{2} \mathrm{O}$. Um den Einfluss zusätzlich im Gas enthaltener Komponenten auf die Entschwefelungsleistung der betrachteten Adsorbentien beschreiben zu können, wurde am Beispiel von Schwefelwasserstoff das binäre Gasgemisch $\left(\mathrm{H}_{2} \mathrm{~S}\right.$ in $\left.\mathrm{CH}_{4}\right)$ zum einen um $\mathrm{CO}_{2}\left(\mathrm{H}_{2} \mathrm{~S}+\mathrm{CO}_{2}\right.$ in $\left.\mathrm{CH}_{4}\right)$ und zum anderen um $\mathrm{H}_{2} \mathrm{O}\left(\mathrm{H}_{2} \mathrm{~S}+\mathrm{H}_{2} \mathrm{O}\right.$ in $\mathrm{CH}_{4}$ ) erweitert. Für die im Folgenden gezeigten Adsorptionsisothermen wurde in den Durchbruchskurvenmessungen einer Messreihe die Konzentration des Schwefelwasserstoffs wie für die Untersuchungen binärer Stoffsysteme im Untersuchungsbereich variiert. Die Konzentration der zusätzlich in der Gasmischung enthaltenen Verbindung $\left(\mathrm{CO}_{2}\right.$ oder $\left.\mathrm{H}_{2} \mathrm{O}\right)$ wurde in diesen Experimenten konstant gehalten.

\subsection{2 $\mathrm{H}_{2} \mathrm{~S}$-Adsorptionsisothermen an Silica-Alumina-Gel}

\section{$\mathrm{H}_{2}$ S-Adsorptionsisothermen bei unterschiedlichen $\mathrm{CO}_{2}$-Konzentrationen in der Gasmischung}

Abbildung 29 zeigt $\mathrm{H}_{2} \mathrm{~S}$ Adsorptionsisothermen bei unterschiedlichen $\mathrm{CO}_{2}$-Konzentrationen in der Gasmischung. Zum Vergleich ist die $\mathrm{H}_{2} \mathrm{~S}$-Isotherme aus binärer Gasmischung $\left(\mathrm{H}_{2} \mathrm{~S}+\right.$ $0 \% \mathrm{CO}_{2}$ ) mit dargestellt. 


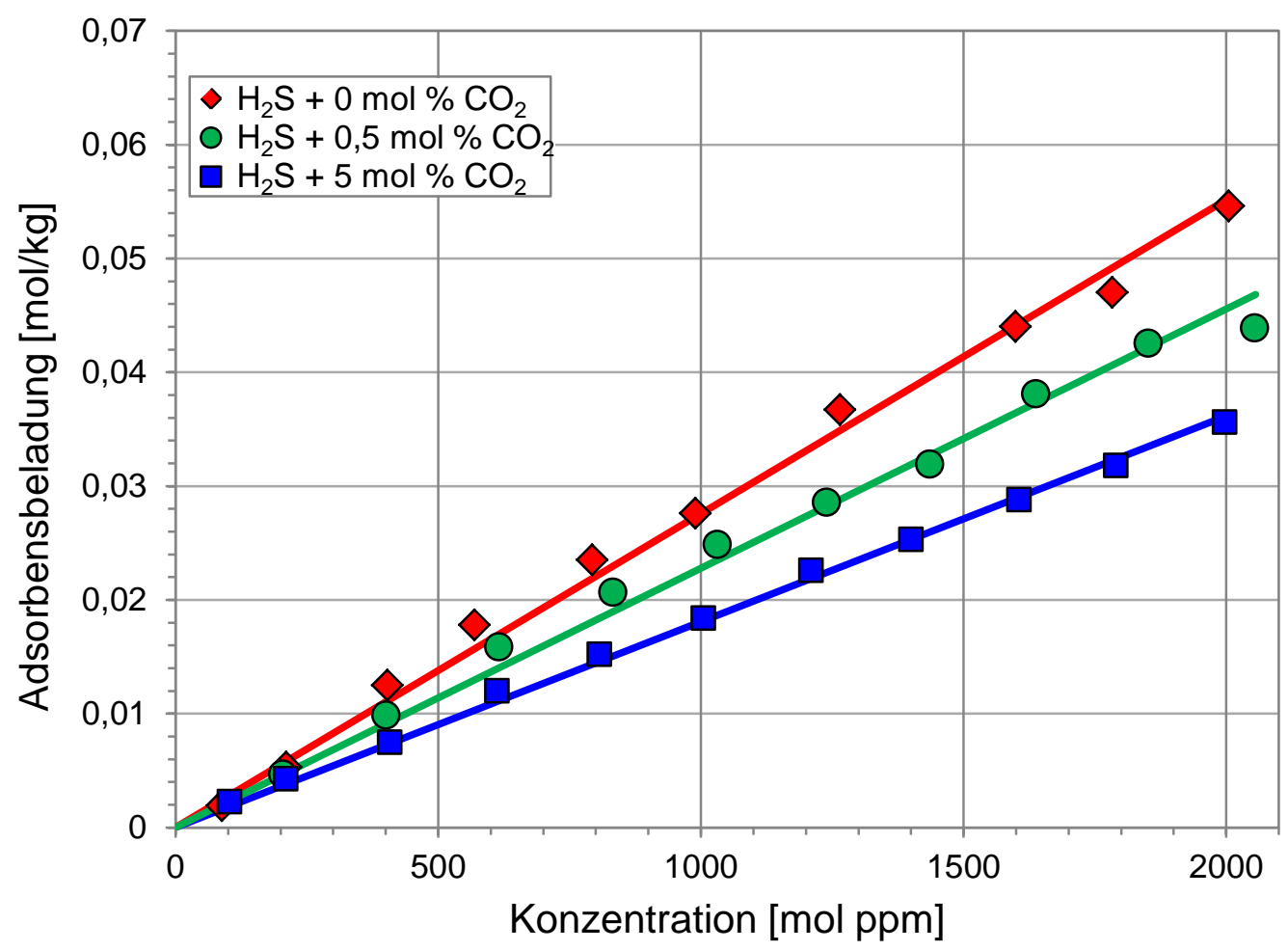

Abbildung 29: Isothermen für die Adsorption von $\mathrm{H}_{2} \mathrm{~S}$ aus $\mathrm{CH}_{4}$ am Silica-Alumina-Gel bei verschiedenen $\mathrm{CO}_{2}$-Konzentrationen in der Gasmischung

Aus der Darstellung wird ersichtlich, dass sich die $\mathrm{H}_{2} \mathrm{~S}$-Kapazität des Silica-Alumina-Gels in Anwesenheit von Kohlendioxid verringert. Bei einer Konzentration von 0,5 mol \% $\mathrm{CO}_{2}$ in der Gasmischung ist ein Rückgang von ca. $17 \%$ bezogen auf die $\mathrm{H}_{2} \mathrm{~S}-$ Kapazität ohne $\mathrm{CO}_{2}$ in der Mischung zu verzeichnen. Bei einer Verzehnfachung der $\mathrm{CO}_{2}-$ Konzentration auf $5 \mathrm{~mol} \%$ verringert sich die $\mathrm{H}_{2} \mathrm{~S}$-Kapazität um weitere $17 \%$. Trotz der Anwesenheit des $\mathrm{CO}_{2}$ ließ sich das Adsorptionsverhalten des $\mathrm{H}_{2} \mathrm{~S}$ durch die Henry-Gleichung beschreiben. Tabelle 19 listet die Henry-Koeffizienten der angepassten Isothermen der ternären Stoffsysteme auf.

Tabelle 19: Parameter der Isothermenanpassungen für die ternären Stoffsysteme $\left(\mathrm{H}_{2} \mathrm{~S}+\mathrm{CO}_{2}\right.$ in $\left.\mathrm{CH}_{4}\right)$ an Silica-Alumina-Gel

\begin{tabular}{|l|c|c|}
\hline Adsorptiv (Matrix) & $\begin{array}{c}\mathbf{k}_{\mathbf{H}} \\
\text { [mol/mg] }\end{array}$ & $\mathbf{R}^{2}$ \\
\hline $\mathrm{H}_{2} \mathrm{~S}\left(0,5 \mathrm{~mol}^{2} \mathrm{CO}_{2}\right.$ in $\left.\mathrm{CH}_{4}\right)$ & $2,27^{\star} 10^{-5}$ & 0.98 \\
\hline $\mathrm{H}_{2} \mathrm{~S}\left(5 \mathrm{~mol} \% \mathrm{CO}_{2}\right.$ in $\left.\mathrm{CH}_{4}\right)$ & $1,807^{\star} 10^{-5}$ & 0.99 \\
\hline
\end{tabular}




\section{$\mathrm{H}_{2}$ S-Adsorptionsisothermen bei unterschiedlichen $\mathrm{H}_{2} \mathrm{O}$-Konzentrationen in der Gasmischung}

In Analogie zu den Untersuchungen der ternären Stoffsysteme $\left(\mathrm{H}_{2} \mathrm{~S}+\mathrm{CO}_{2}\right.$ in $\left.\mathrm{CH}_{4}\right)$ wurde ebenfalls am Beispiel von $\mathrm{H}_{2} \mathrm{~S}$ für beide betrachtete Adsorbentien der Einfluss von Wasser auf die Entschwefelungsleistung untersucht. Die $\mathrm{H}_{2} \mathrm{~S}$-Konzentration wurde in diesen Experimenten wiederum im Bereich 0-2000 mol ppm variiert, während die $\mathrm{H}_{2} \mathrm{O}$-Konzentration konstant gehalten wurde. Es wurden Messreihen bei 500 und $1000 \mathrm{~mol} \mathrm{ppm} \mathrm{H}_{2} \mathrm{O}$ durchgeführt. Abbildung 30 zeigt die aus diesen Experimenten bestimmten $\mathrm{H}_{2} \mathrm{~S}$ Beladungskapazitäten.

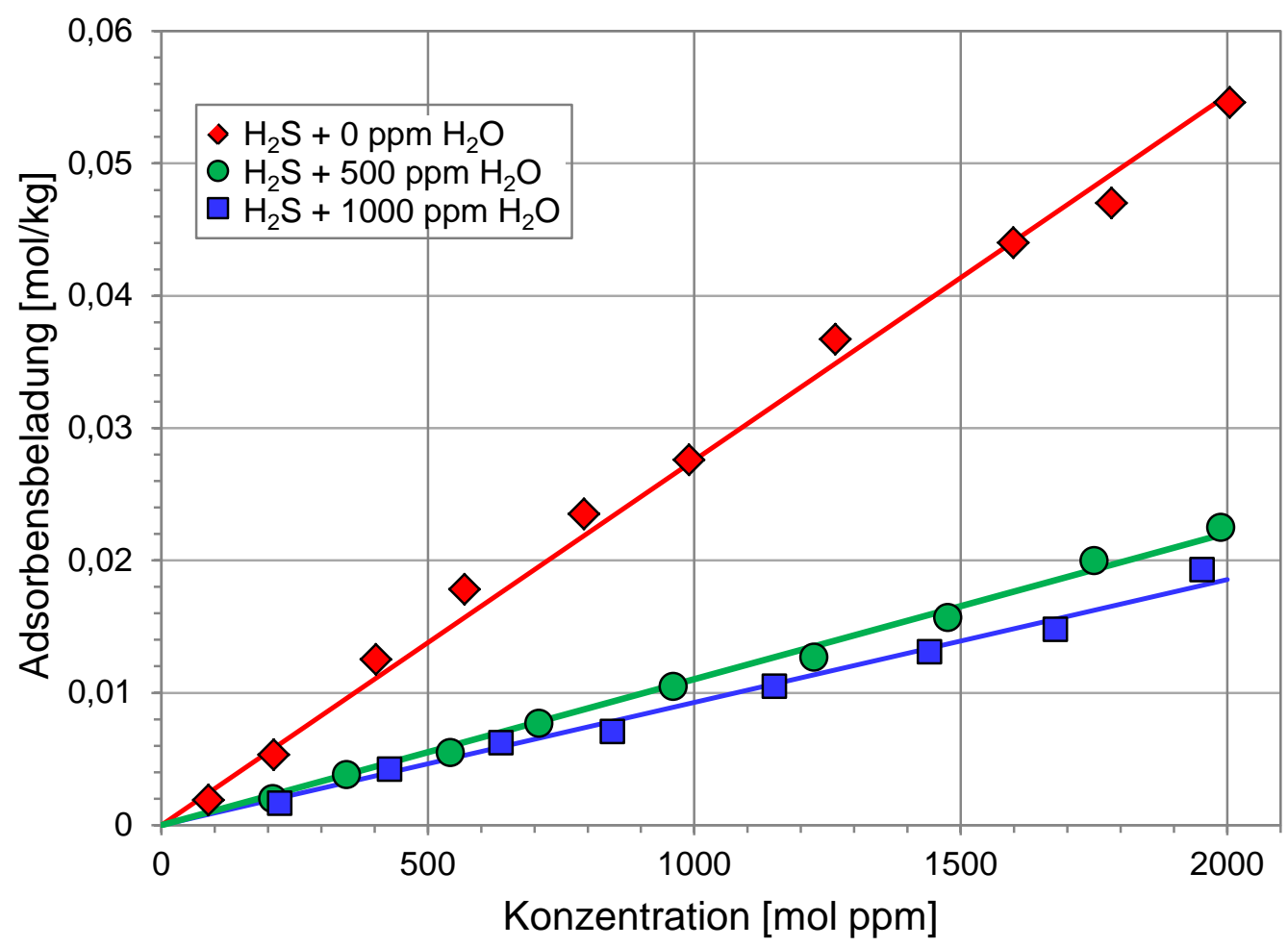

Abbildung 30: Isothermen für die Adsorption von $\mathrm{H}_{2} \mathrm{~S}$ aus $\mathrm{CH}_{4}$ am Silica-Alumina-Gel bei unterschiedlichen Wasserkonzentrationen in der Gasmischung

Es zeigte sich, dass es in Anwesenheit von $\mathrm{H}_{2} \mathrm{O}$ ebenfalls zu einer deutlichen Verminderung der $\mathrm{H}_{2} \mathrm{~S}$-Kapazitäten kommt. Für eine $\mathrm{H}_{2} \mathrm{O}$-Konzentration von $500 \mathrm{~mol}$ ppm in der Gasmischung kommt es im Vergleich zur Messreihe ohne Wasser in der Gasmischung zu einem Kapazitätsrückgang für Schwefelwasserstoff von ca. $60 \%$. Eine Verdopplung der Wasserkonzentration hat einen weiteren Kapazitätsverlust von ca. 6 \% zur Folge. Tabelle 20 gibt eine Auflistung der Isothermenparameter sowie der zugehörigen Bestimmtheitsmaße. 
Tabelle 20: Parameter der Isothermenanpassungen für die ternären Stoffsysteme $\left(\mathrm{H}_{2} \mathrm{~S}+\mathrm{CO}_{2}\right.$ in $\left.\mathrm{CH}_{4}\right)$ an Silica-Alumina-Gel

\begin{tabular}{|l|c|c|}
\hline Adsorptiv (Matrix) & $\begin{array}{c}\mathbf{k}_{\mathrm{H}} \\
{[\mathrm{mol} / \mathrm{mg}]}\end{array}$ & $\mathbf{R}^{2}$ \\
\hline $\mathrm{H}_{2} \mathrm{~S}\left(500 \mathrm{~mol} \mathrm{ppm} \mathrm{H}_{2} \mathrm{O}\right.$ in $\left.\mathrm{CH}_{4}\right)$ & $1,103^{*} 10^{-5}$ & 0,99 \\
\hline $\mathrm{H}_{2} \mathrm{~S}\left(1000 \mathrm{~mol} \mathrm{ppm} \mathrm{H}_{2} \mathrm{O}\right.$ in $\left.\mathrm{CH}_{4}\right)$ & $9,27 * 10^{-6}$ & 0,98 \\
\hline
\end{tabular}

\section{$\mathrm{H}_{2}$ S-Adsorptionsisothermen bei unterschiedlichen $\mathrm{CO}_{2}$-Konzentrationen in der Gasmischung an modifizierten Silica-Alumina-Gelen}

Die Untersuchungen mit den modifizierten Silica-Alumina-Gelen wurden analog zu den in Abbildung 29 gezeigten Isothermen für das Silica-Alumina-Gel-Standardmaterial durchgeführt. Abbildung 31 und Abbildung 32 zeigen die $\mathrm{H}_{2} \mathrm{~S}$ Isothermen bei unterschiedlichem $\mathrm{CO}_{2^{-}}$ Gehalt in der Gasmischung.

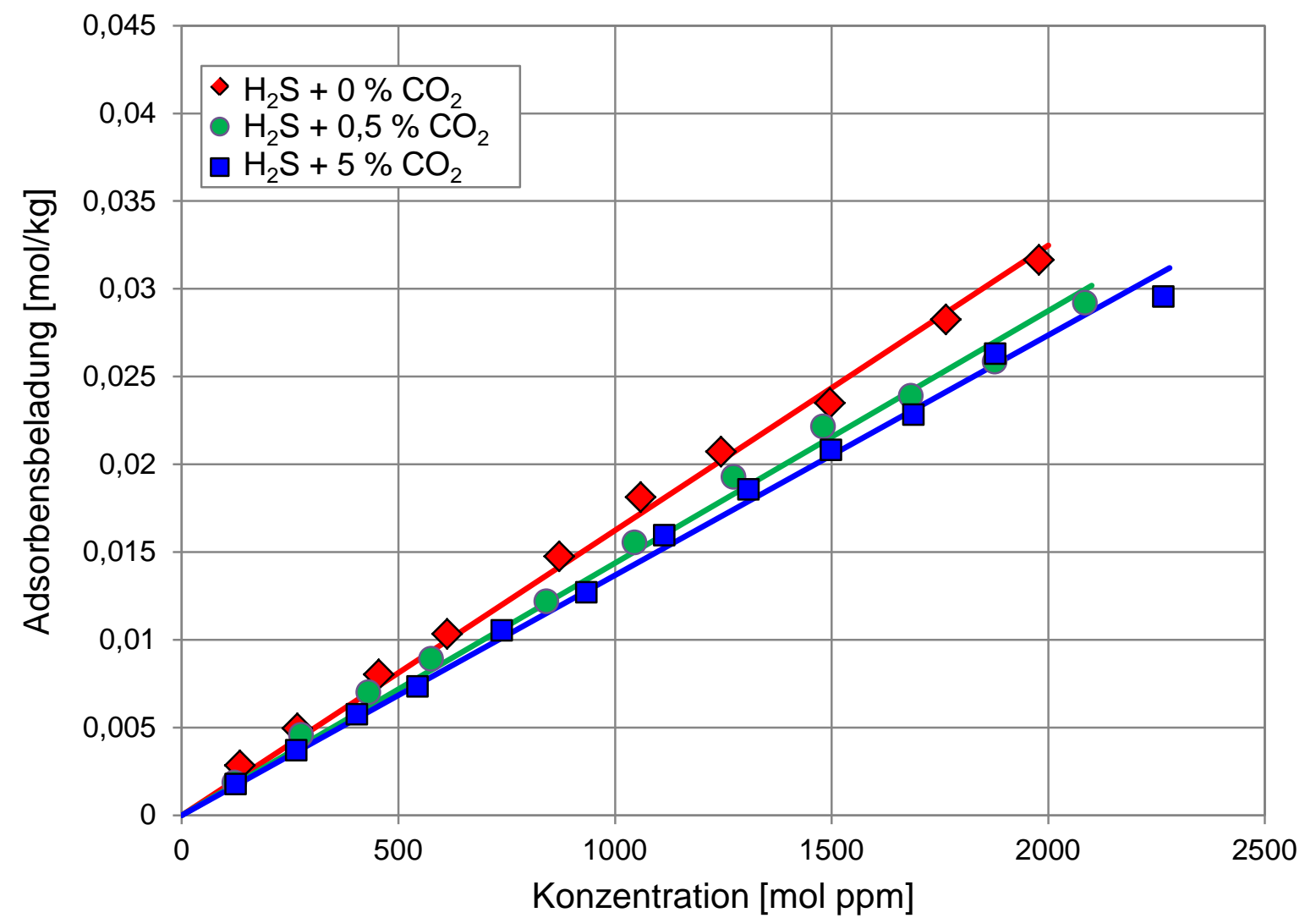

Abbildung 31: Isothermen für die Adsorption von $\mathrm{H}_{2} \mathrm{~S}$ aus $\mathrm{CH}_{4}$ an der Silica-Alumina-Gel-Variante mit 1,5\% Al-Gehalt bei unterschiedlichen $\mathrm{CO}_{2}$-Konzentrationen in der Gasmischung 


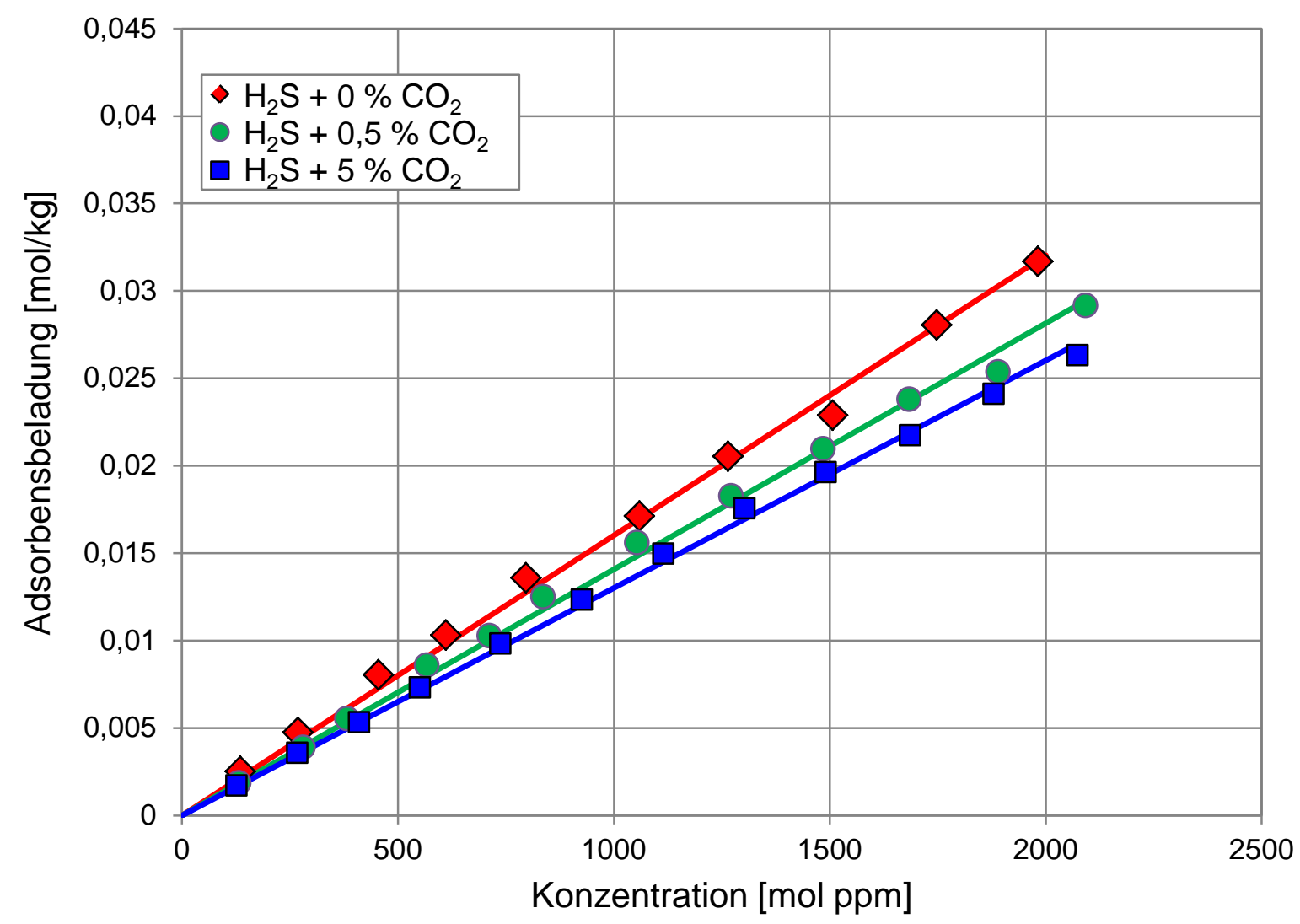

Abbildung 32: Isothermen für die Adsorption von $\mathrm{H}_{2} \mathrm{~S}$ aus $\mathrm{CH}_{4}$ an der Silica-Alumina-Gel-Variante mit $0,3 \% \mathrm{Al}$-Gehalt bei unterschiedlichen $\mathrm{CO}_{2}$-Konzentrationen in der Gasmischung

Aus den beiden Abbildungen wird ersichtlich, dass die Adsorbentien ein sehr ähnlich Adsorptionsverhalten zeigen. Im Vergleich zum binären Stoffsystem bewirkt eine Konzentration von $0,5 \mathrm{~mol} \%$ in der Gasmischung einen Kapazitätsrückgang von ungefähr $11 \%$ (SilicaAlumina-Gel mit 1,5 Al-Gehalt) bzw. $12 \%$ (Silica-Alumina-Gel mit 0,3\% Al-Gehalt): Aus einer Verzehnfachung der $\mathrm{CO}_{2}$-Konzentration (0,5 mol \% => $\left.5 \mathrm{~mol} \%\right)$ ergibt sich ein zusätz-

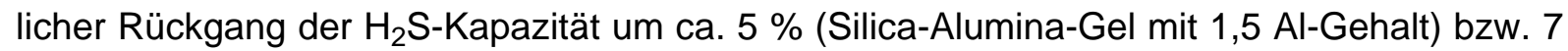
\% (Silica-Alumina-Gel mit 0,3 \% Al-Gehalt). Tabelle 21 gibt einen Überblick über die angepassten Isothermenparameter.

Tabelle 21: Parameter der Isothermenanpassungen für die ternären Stoffsysteme $\left(\mathrm{H}_{2} \mathrm{~S}+\mathrm{CO}_{2}\right.$ in $\left.\mathrm{CH}_{4}\right)$ an den modifizierten Silica-Alumina-Gel-Varianten

\begin{tabular}{|c|c|c|c|}
\hline Adsorbens & Adsorptiv (Matrix) & $\begin{array}{c}\mathbf{k}_{\mathrm{H}} \\
{[\mathrm{mol} / \mathrm{mg}]}\end{array}$ & $\mathbf{R}^{2}$ \\
\hline $\begin{array}{l}\text { Silica-Alumina-Gel } \\
\text { (ca. } 1,5 \% \text { A)l }\end{array}$ & $\begin{array}{l}\mathrm{H}_{2} \mathrm{~S}\left(0,5 \mathrm{~mol} \% \mathrm{CO}_{2} \text { in } \mathrm{CH}_{4}\right) \\
\mathrm{H}_{2} \mathrm{~S}\left(5 \mathrm{~mol} \% \mathrm{CO}_{2} \text { in } \mathrm{CH}_{4}\right)\end{array}$ & $\begin{array}{l}1,44 * 10^{-5} \\
1,37 * 10^{-5}\end{array}$ & $\begin{array}{l}0,99 \\
0,99\end{array}$ \\
\hline $\begin{array}{l}\text { Silica-Alumina-Gel } \\
\text { (ca. } 0,3 \% \mathrm{Al} \text { ) }\end{array}$ & $\begin{array}{l}\mathrm{H}_{2} \mathrm{~S}\left(0,5 \mathrm{~mol} \% \mathrm{CO}_{2} \text { in } \mathrm{CH}_{4}\right) \\
\mathrm{H}_{2} \mathrm{~S}\left(5 \mathrm{~mol} \% \mathrm{CO}_{2} \text { in } \mathrm{CH}_{4}\right)\end{array}$ & $\begin{array}{l}1,408 * 10^{-5} \\
1,301 * 10^{-5}\end{array}$ & $\begin{array}{l}0,99 \\
0,99\end{array}$ \\
\hline
\end{tabular}




\subsection{3 $\mathrm{H}_{2} \mathrm{~S}$-Adsorptionsisothermen an 5A Zeolith}

$\mathrm{H}_{2} \mathrm{~S}$-Ads orptions is othermen bei unterschiedlichen $\mathrm{CO}_{2}$-Konzentrationen in der Gasmischung

Abbildung 33 zeigt $\mathrm{H}_{2} \mathrm{~S}$-Isothermen für die Adsorption aus dem binären $\left(\mathrm{H}_{2} \mathrm{~S}\right.$ in $\left.\mathrm{CH}_{4}\right)$ und den ternären $\left(\mathrm{H}_{2} \mathrm{~S}+\mathrm{CO}_{2}\right.$ in $\left.\mathrm{CH}_{4}\right)$ Gasgemischen am 5A Zeolith.

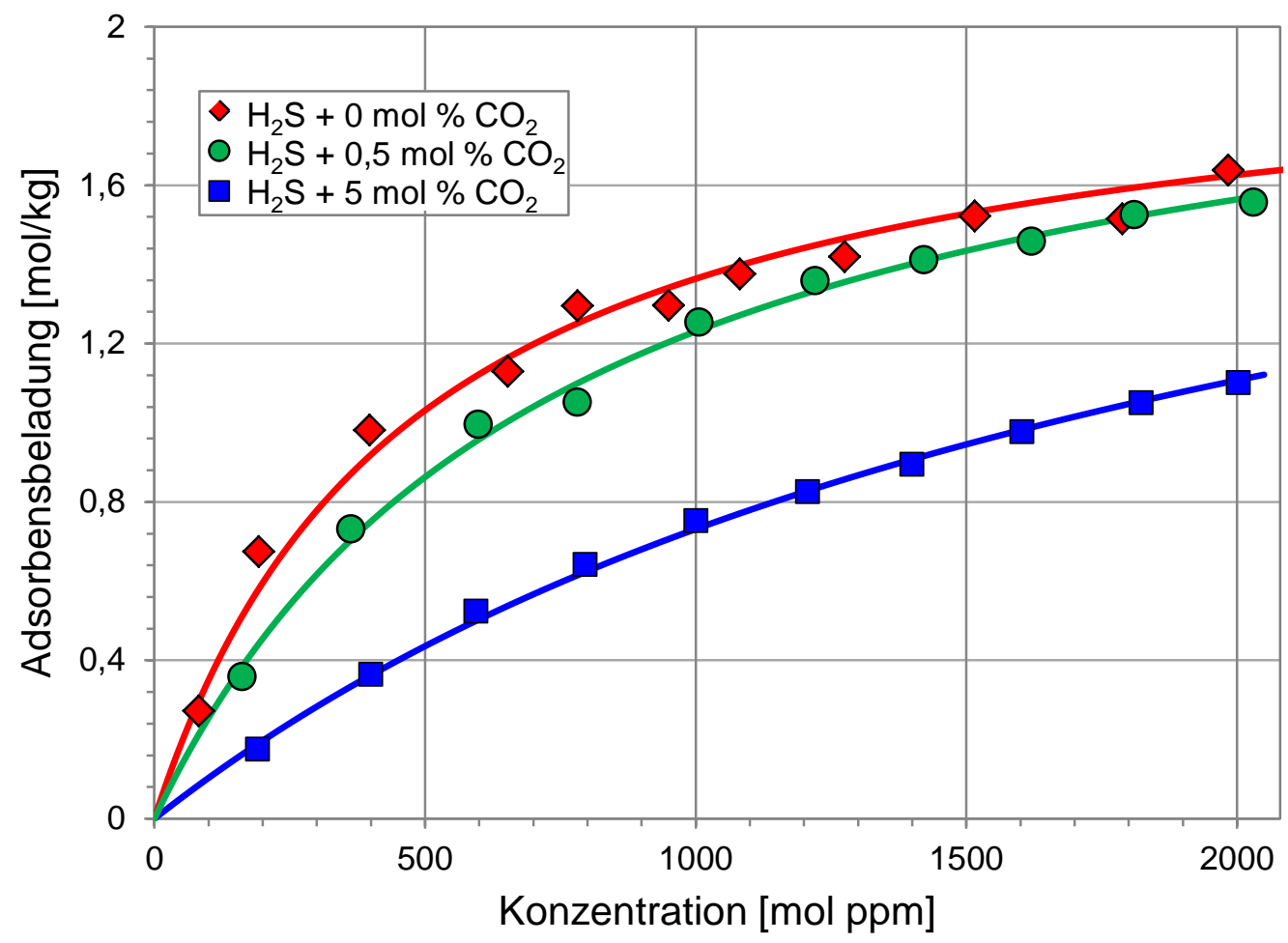

Abbildung 33: Isothermen für die Adsorption von $\mathrm{H}_{2} \mathrm{~S}$ aus $\mathrm{CH}_{4}$ am Zeolith des Typs $5 \mathrm{~A}$ bei unterschiedlichen $\mathrm{CO}_{2}$-Konzentrationen in der Gasmischung

Die Isothermendarstellung verdeutlicht, dass sich die $\mathrm{H}_{2} \mathrm{~S}$-Kapazität des $5 \mathrm{~A}$-Molsiebs in Anwesenheit von $\mathrm{CO}_{2}$ für beide betrachteten $\mathrm{CO}_{2}$-Konzentrationen in der Gasmischung verringert. Für den Fall des geringeren $\mathrm{CO}_{2}$-Anteils $(0,5 \mathrm{~mol} \%)$ in der Gasmischung zeigt sich ein geringer Abfall in der $\mathrm{H}_{2} \mathrm{~S}$-Kapazität. Für Experimente im Bereich von $2000 \mathrm{~mol}$ ppm $\mathrm{H}_{2} \mathrm{~S}$ ergibt sich ein Abfall in der $\mathrm{H}_{2} \mathrm{~S}$-Beladung von ca. 4\%. Wird der $\mathrm{CO}_{2}$-Gehalt in der Mischung deutlich erhöht $(0,5=>5 \mathrm{~mol} \%)$, ist auch der Kapazitätsrückgang für das Adsorptiv $\mathrm{H}_{2} \mathrm{~S}$ stärker ausgeprägt. Für diese Messreihe beträgt der $\mathrm{H}_{2} \mathrm{~S}$-Kapazitätsverlust bezogen auf das binäre Stoffsystem im hohen Untersuchungsbereich ca. 32 \%. Trotz der reduzierten $\mathrm{H}_{2} \mathrm{~S}$ Beladungskapazitäten ließen sich die betrachteten Systeme ebenfalls in sehr guter Nähe- 
rung durch die Langmuir-Gleichung beschreiben. Tabelle 22 gibt einen Überblick über die angepassten Parameter.

Tabelle 22: Parameter der Isothermenanpassungen für die ternären Stoffsysteme $\left(\mathrm{H}_{2} \mathrm{~S}+\mathrm{CO}_{2}\right.$ in $\left.\mathrm{CH}_{4}\right)$ am Zeolith 5A

\begin{tabular}{|l|c|c|c|}
\hline Adsorptiv (Matrix) & $\begin{array}{c}\mathbf{X}_{\text {mon }} \\
{[\mathrm{mol} / \mathbf{k g}]}\end{array}$ & $\begin{array}{c}\mathbf{b} \\
{[\mathbf{b}]}\end{array}$ & $\mathbf{R}^{2}$ \\
\hline $\mathrm{H}_{2} \mathrm{~S}\left(0,5 \mathrm{~mol}^{2} \mathrm{CO}_{2}\right.$ in $\left.\mathrm{CH}_{4}\right)$ & 2,145 & $1,35^{\star} 10^{-3}$ & 0,99 \\
\hline $\mathrm{H}_{2} \mathrm{~S}\left(5 \mathrm{~mol}_{2} \mathrm{CO}_{2}\right.$ in $\left.\mathrm{CH}_{4}\right)$ & 2,278 & $4,73^{\star} 10^{-4}$ & 0,98 \\
\hline
\end{tabular}

$\mathrm{H}_{2} \mathrm{~S}$-Ads orptions is othermen bei unterschiedlichen $\mathrm{H}_{2} \mathrm{O}$-Konzentrationen in der Gasmischung

Abbildung 34 zeigt die ermittelten $\mathrm{H}_{2} \mathrm{~S}$-Isothermen für die Adsorption aus dem ternären Stoffsystem $\left(\mathrm{H}_{2} \mathrm{~S}+\mathrm{H}_{2} \mathrm{O}\right.$ in $\left.\mathrm{CH}_{4}\right)$ am Zeolith des Typs 5A. Zu Vergleichszwecken ist wiederum die $\mathrm{H}_{2} \mathrm{~S}$-Isotherme für die Adsorption aus dem binären Stoffsystem mit aufgeführt.

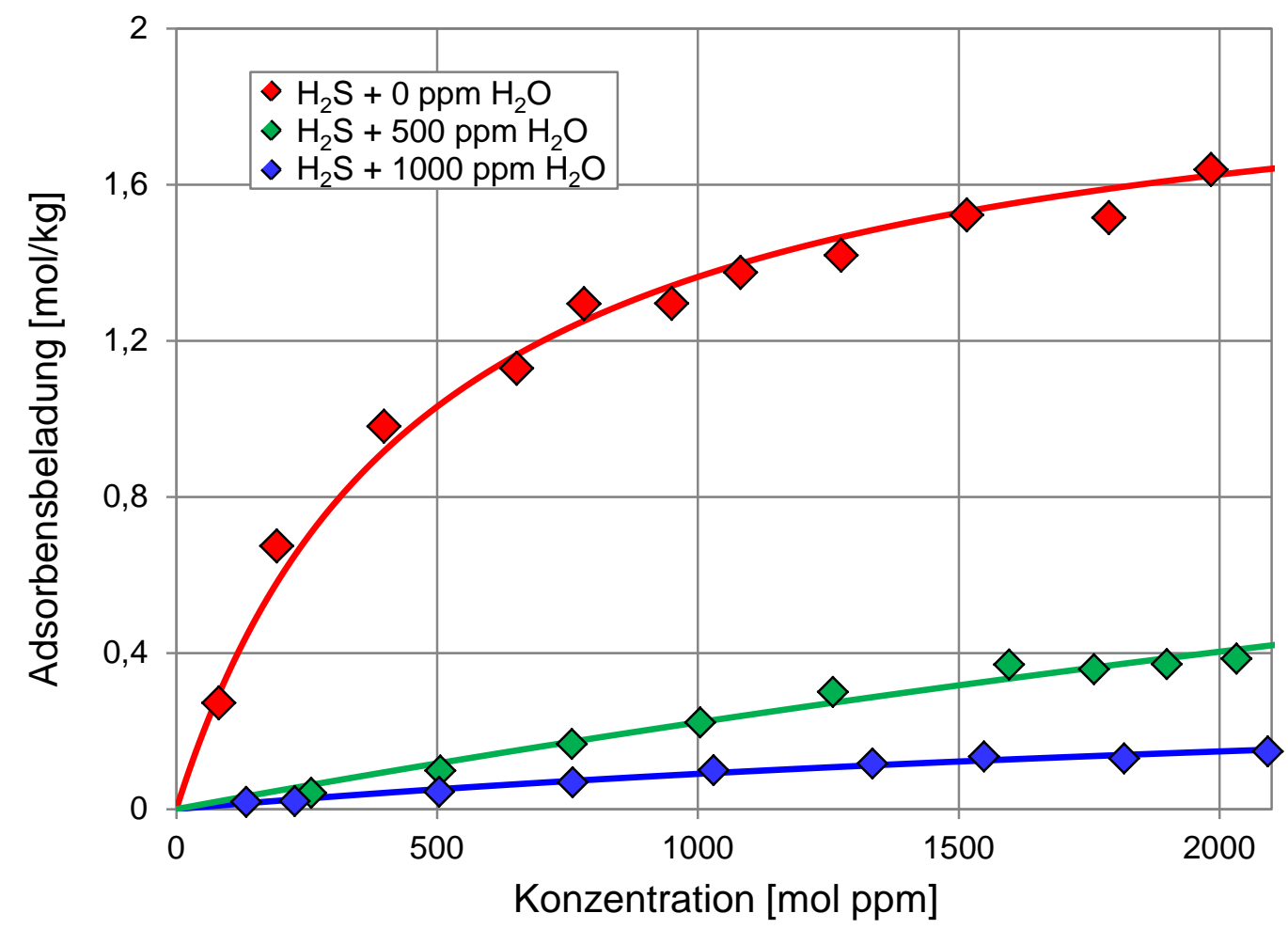

Abbildung 34: Isothermen für die Adsorption von $\mathrm{H}_{2} \mathrm{~S}$ aus $\mathrm{CH}_{4}$ am Zeolith des Typs $5 \mathrm{~A}$ bei unterschiedlichen $\mathrm{H}_{2} \mathrm{O}$-Konzentration in der Gasmischung 
Es zeigte sich ein starker Rückgang in der $\mathrm{H}_{2} \mathrm{~S}$-Kapazität. Für eine $\mathrm{H}_{2} \mathrm{O}$-Konzentration von $500 \mathrm{~mol}$ ppm in der Gasmischung kommt es demnach am rechten Rand des Untersuchungsbereichs (ca. 2000 mol ppm $\mathrm{H}_{2} \mathrm{~S}$ ) zu einem Kapazitätsverlust von 75\% (von ca. 1,6 auf ca. 0,4 mol/kg). Eine Verdopplung des Wassergehaltes auf $1000 \mathrm{~mol}$ ppm in der Gasmischung bewirkt einen Rückgang von ca. 90\% (von 1,6 mol/kg auf ca. 0,16 mol/kg) bezogen auf die Kapazitäten des binären Stoffsystems. Tabelle 23 gibt eine Auflistung der Anpassungsparameter der Langmuir-Gleichung sowie der Anpassungsgüte ausgedrückt durch das Bestimmtheitsmaß $R^{2}$.

Tabelle 23: Angepasste Isothermenparameter für die Stoffsysteme $\mathrm{H}_{2} \mathrm{~S}+\mathrm{H}_{2} \mathrm{O}$ in $\mathrm{CH}_{4}$ am Zeolith $5 \mathrm{~A}$

\begin{tabular}{|l|c|c|c|}
\hline Adsorptiv (Matrix) & $\begin{array}{c}\mathbf{X}_{\text {mon }} \\
{[\mathrm{mol} / \mathrm{kg}]}\end{array}$ & $\begin{array}{c}\mathbf{b} \\
{[-]}\end{array}$ & $\mathbf{R}^{2}$ \\
\hline $\mathrm{H}_{2} \mathrm{~S}\left(500\right.$ mol-ppm $\mathrm{H}_{2} \mathrm{O}$ in $\left.\mathrm{CH}_{4}\right)$ & 2,178 & $1,136^{\star} 10^{-4}$ & 0,97 \\
\hline $\mathrm{H}_{2} \mathrm{~S}\left(1000\right.$ mol-ppm $\left.\mathrm{H}_{2} \mathrm{Oin} \mathrm{CH}_{4}\right)$ & 0,407 & $2,85^{\star} 10^{-4}$ & 0,98 \\
\hline
\end{tabular}

\subsection{Diskussion der ternären Stoffsysteme}

\subsubsection{Adsorption an Silica-Alumina-Gel}

\section{Einfluss von $\mathrm{CO}_{2}$ auf die $\mathrm{H}_{2} \mathrm{~S}$-Adsorption}

Die Isothermendarstellung (vgl. Abbildung 29) zeigt, dass das zusätzlich in der Gasmischung enthaltene $\mathrm{CO}_{2}$ einen negativen Einfluss auf die Adsorption des $\mathrm{H}_{2} \mathrm{~S}$ hat. Es kommt zu einer Konkurrenzsituation auf der Adsorbensoberfläche, wodurch abhängig von der Konzentration des $\mathrm{CO}_{2}$ ein unterschiedlich stark ausgeprägter $\mathrm{H}_{2} \mathrm{~S}$-Kapazitätsverlust auftritt. Dies lässt sich anhand der in Abbildung 35 dargestellten normierten Durchbruchskurven für Experimente im Bereich bis 2000 mol ppm $\mathrm{H}_{2} \mathrm{~S}$ beschreiben. 


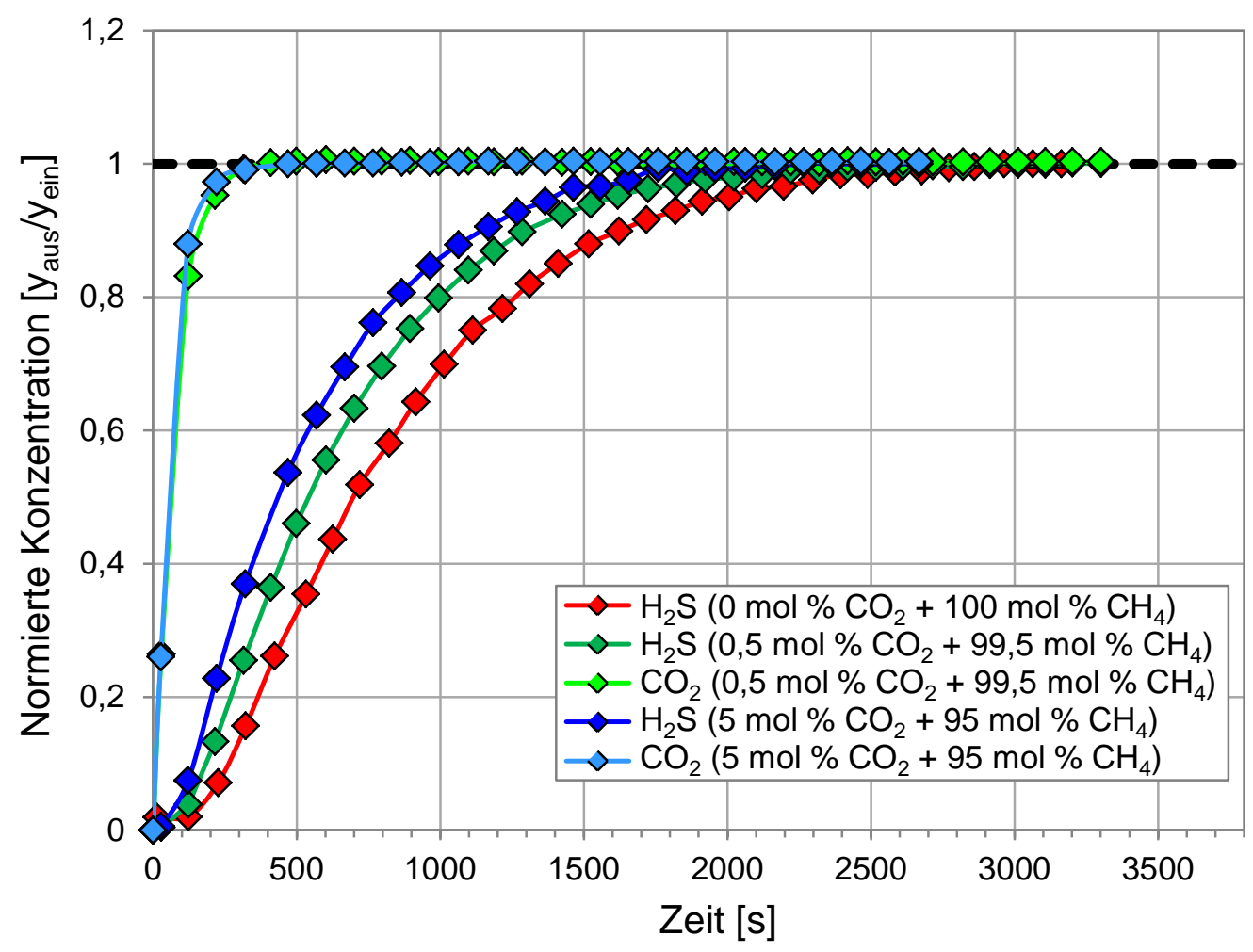

Abbildung 35: Normierte Durchbruchskurven für die Adsorption von $\mathrm{H}_{2} \mathrm{~S}$ und $\mathrm{CO}_{2}$ am Silica-Alumina-

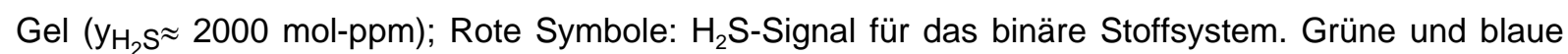
Symbole: Simultan detektierte Signale für $\mathrm{H}_{2} \mathrm{~S}$ (dunkle Farben) und $\mathrm{CO}_{2}$ (helle Farben) für die ternären Stoffsysteme

Im Falle der Experimente für die ternären Stoffsysteme durchläuft die Konzentrationsfront des unpolaren $\mathrm{CO}_{2}$ mit der niedrigen Affinität zur polaren Oberfläche des Silica-Alumina-Gels und einer schnellen Kinetik die Schüttung (hell blaue und hell grüne Kurve). Im Gegensatz dazu zeigt $\mathrm{H}_{2} \mathrm{~S}$ eine deutlich höhere Affinität zum Adsorbens. Die entsprechenden Fronten verlaufen flacher und langsamer durch die Schüttung (dunkel grüne und dunkel blaue Kurve). Auf Grund der schnellen Kinetik der $\mathrm{CO}_{2}$-Fronten zeigt sich keine Beeinflussung dieser durch die zeitlich später durchbrechenden $\mathrm{H}_{2} \mathrm{~S}$-Fronten. Eine Vertiefung der Diskussion kinetischer Effekte ternärer Stoffsysteme findet sich in Abschnitt 5.4.

Auf Grund des schnell adsorbierenden $\mathrm{CO}_{2}$ entsteht eine Vorbeladung der Adsorbensoberfläche mit adsorbierten $\mathrm{CO}_{2}$-Molekülen. Dadurch wird die Anzahl an freien Adsorptionsplätzen verringert, so dass die später durchbrechende $\mathrm{H}_{2} \mathrm{~S}$-Konzentrationsfront zum einen steiler verläuft und zum anderen früher das stoffliche Gleichgewicht erreicht als im Falle der Messung ohne $\mathrm{CO}_{2}$ (rote Kurve). In der Isothermendarstellung entsprechender 
Systeme (vgl. Abbildung 33) äußert sich dies abhängig von der $\mathrm{CO}_{2}$-Konzentration durch geringere Kapazitäten. Auf Grund der allgemein sehr geringen Kapazitäten konnten für beide ternären Stoffsysteme keine Verdrängungseffekte beobachtet werden, die sich in einer Durchbruchskurvenmessung in einem Anstieg der Konzentration über die Eingangskonzentration hinaus zeigen würden. Die in der Literatur $[61,83]$ beschriebene Bildung von Carbonylsulfid entsprechend dem Gleichgewicht

$$
\mathrm{H}_{2} \mathrm{~S}+\mathrm{CO}_{2} \rightleftharpoons \mathrm{COS}+\mathrm{H}_{2} \mathrm{O}
$$

konnte unter den gewählten Versuchsbedingungen nicht beobachtet werden.

Entsprechend der für das binäre Stoffsystem $\left(\mathrm{CO}_{2}\right.$ in $\left.\mathrm{CH}_{4}\right)$ gefundenen Henry-Isotherme sollte eine Verzehnfachung des $\mathrm{CO}_{2}$-Gehaltes beim Schritt von 0,5 mol \% zu $5 \mathrm{~mol} \%$ in der Gasmischung eine Erhöhung der $\mathrm{CO}_{2}$-Beladung um den Faktor 10 zur Folge haben. Dementsprechend würde man für die Messreihe mit höherer $\mathrm{CO}_{2}$-Konzentration einen deutlich ausgeprägteren Kapazitätsverlust für $\mathrm{H}_{2} \mathrm{~S}$ erwarten. Der zusätzlich beobachtete Kapazitätsrückgang entspricht jedoch nur gerade demjenigen, der für den Sprung von 0 auf 0,5 mol \% $\mathrm{CO}_{2}$ in der Gasmischung nachgewiesen werden konnte. Dieser Effekt würde der unter Abschnitt 4.2.1 diskutierten Annahme entsprechen, dass ein bestimmter Anteil der zur Verfügung stehenden Adsorptionsplätze lediglich mit dem Schwefelwasserstoff wechselwirkt. Das im Silica-Alumina-Gel enthaltene Aluminiumoxid nimmt hierbei eine besondere Rolle ein. Um diesen Zusammenhang genauer zu betrachten, wurden die ternären Untersuchungen ebenfalls mit den modifizierten Silica-Alumina-Gelen durchgeführt.

\section{Modifizierte Silica-Alumina-Gele}

Abbildung 36 zeigt die $\mathrm{H}_{2} \mathrm{~S}$-Adsorptionsisothermen aller drei Silica-Alumina-Gel-Varianten bei verschiedenen $\mathrm{CO}_{2}$-Konzentrationen in der Gasmischung (vgl. Abbildung 29, Abbildung 31 und Abbildung 32). 


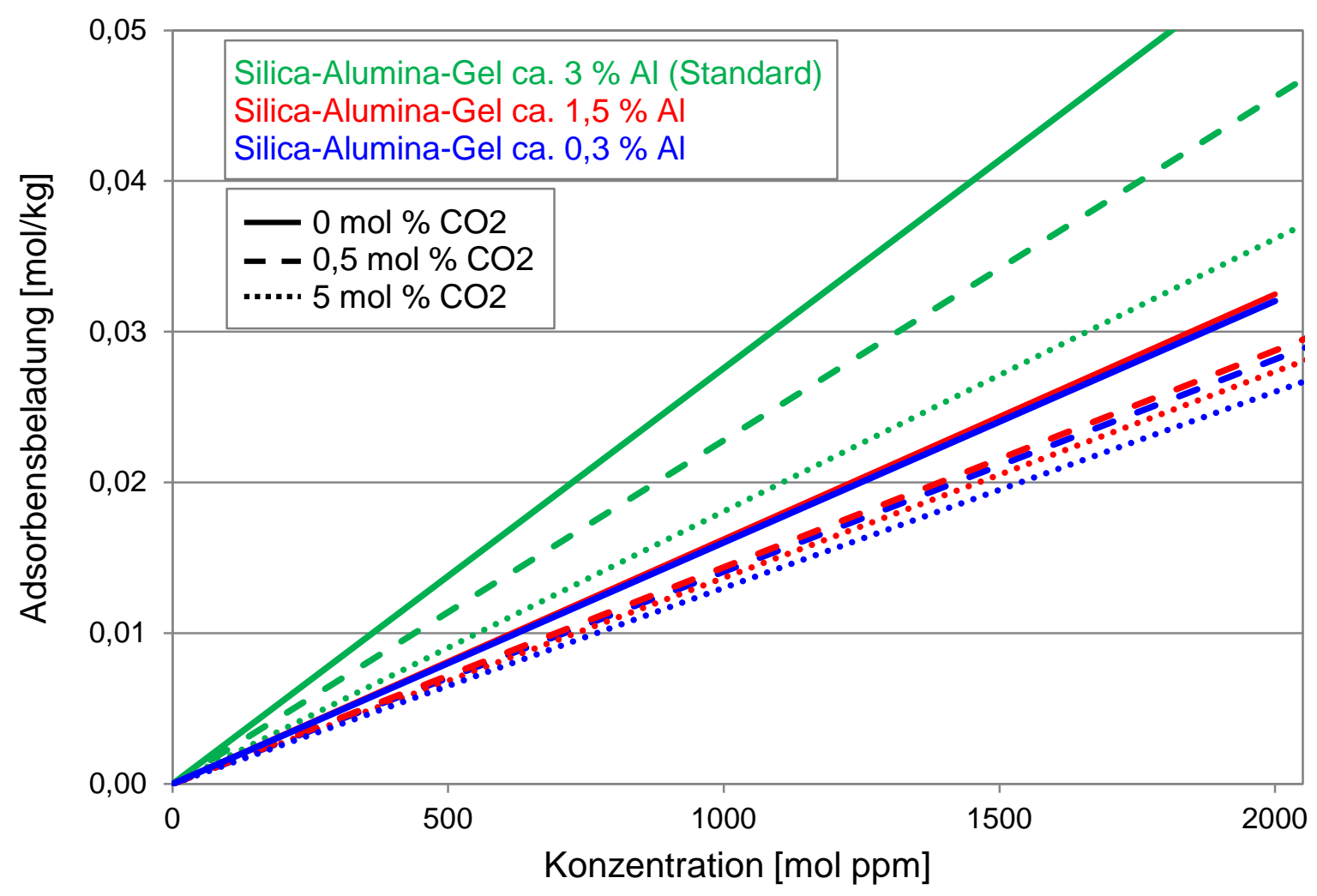

Abbildung 36: $\mathrm{H}_{2} \mathrm{~S}$-Adsorptionsisothermen für Silica-Alumina-Gel-Varianten bei verschiedenen $\mathrm{CO}_{2}$ Konzentrationen in der Gasmischung

Zur besseren Übersicht sind in dieser Darstellung lediglich die angepassten HenryIsothermen für die $\mathrm{H}_{2} \mathrm{~S}$-Adsorption aus den Stoffsystemen $\left(\mathrm{H}_{2} \mathrm{~S}+5 \mathrm{~mol} \% \mathrm{CO}_{2}\right.$ in $\mathrm{CH}_{4}, \mathrm{H}_{2} \mathrm{~S}$ $+0,5 \mathrm{~mol} \% \mathrm{CO}_{2}$ in $\left.\mathrm{CH}_{4}\right)$ im Vergleich zur Messung aus dem Stoffgemisch $\left(\mathrm{H}_{2} \mathrm{~S}\right.$ in $\left.\mathrm{CH}_{4}\right)$ enthalten. Für alle drei Materialien zeigt sich in Anwesenheit des zweiten Adsorptivs $\mathrm{CO}_{2}$ ein unterschiedlich stark ausgeprägter Verlust der $\mathrm{H}_{2} \mathrm{~S}$-Kapazität. Eine Erhöhung des $\mathrm{CO}_{2^{-}}$ Gehaltes in der Gasmischung hat einen zusätzlichen $\mathrm{H}_{2} \mathrm{~S}$-Kapazitätsverlust zur Folge.

Die Verringerung der $\mathrm{H}_{2} \mathrm{~S}$-Kapazitäten kann durch die Änderung der Vorbeladung durch das ebenfalls adsorbierte Kohlendioxid erklärt werden. Bestimmte Adsorptionsplätze, die dem $\mathrm{H}_{2} \mathrm{~S}$ bei der Adsorption aus Methan ohne $\mathrm{CO}_{2}$ zur Verfügung stehen, können bei der simultanen Adsorption von $\mathrm{H}_{2} \mathrm{~S}$ und $\mathrm{CO}_{2}$ nicht mehr vom $\mathrm{H}_{2} \mathrm{~S}$ besetzt werden. Zwischen dem Adsorptionsverhalten des Standardmaterials und dem der modifizierten Silica-Alumina-Gele bestehen jedoch wesentliche Unterschiede. Zum einen sind die erreichten Kapazitäten der modifizierten Materialien deutlich geringer als für das Standardmaterial. Zum anderen sind die gefundenen Kapazitäten der untersuchten Stoffsysteme im Gegensatz zum Standardmaterial an den modifizierten Materialen sehr ähnlich. Weiterhin ist der Kapazitätsverlust im 
Falle der ternären Stoffgemische verglichen mit der jeweiligen Beladung des binären Systems für die modifizierten Gele geringer.

Für das Standardmaterial lässt dies die Vermutung zu, dass der Kapazitätsverlust für $\mathrm{H}_{2} \mathrm{~S}$ mit einer teilweisen Besetzung von Silanol-Plätzen durch $\mathrm{CO}_{2}$ begründet werden kann. Wie in Abschnitt 4.2.1 gezeigt wurde, stehen die Aluminiumoxid-Plätze jedoch nur dem $\mathrm{H}_{2} \mathrm{~S}$ als Bindungsstellen zur Verfügung. Dies könnte der Grund dafür sein, dass der zusätzliche Kapazitätsverlust für $\mathrm{H}_{2} \mathrm{~S}$ infolge einer Verzehnfachung der $\mathrm{CO}_{2}$-Konzentration nur vergleichsweise gering ausfällt, da $\mathrm{H}_{2} \mathrm{~S}$ vorwiegend mit den Al-Plätzen wechselwirkt.

Das Adsorptionsverhalten der modifizierten Materialien lässt sich anhand dieser Argumentation nicht erklären. Es zeigen sich zwei widersprüchliche Effekte, die zu einer anderen Hypothese führen. Denn obwohl sich der Aluminium-Gehalt der modifizierten Gele deutlich unterscheidet (1,5\% bzw. 0,3\%), ist deren Adsorptionsverhalten sehr ähnlich.

Dieses Verhalten ist auf der Grundlage gesicherter Erkenntnisse nicht zu erklären. Es bietet sich aber eine spekulative Deutung an:

Festkörper-NMR Untersuchungen des Herstellers konnten zeigen, dass es bei der Synthese des Silica-Alumina-Gels zur Ausbildung zweier unterschiedlicher Aluminiumkonfigurationen kommt. Es wurden eine tetraedrische und eine oktaedrische Anordnung des Aluminiums in der Matrix nachgewiesen [84].

Eine Adsorption könnte entsprechend dem in Abschnitt 4.2.1 erläuterten Lewis-Säure-BaseMechanismus bevorzugt mit dem elektronisch nicht abgesättigten tetraedrisch orientierten Aluminium stattfinden. Die oktaedrisch koordinierten Aluminiumplätze zeigen aufgrund ihrer elektronischen Absättigung womöglich keine starke Selektivität für $\mathrm{H}_{2} \mathrm{~S}$.

Es könnte bei der Erhöhung des Al-Gehaltes bei der Synthese verschiedener Gele einen Schwellenwert geben, ab welchem sich vorwiegend solche unselektiven Aluminiumplätze ausbilden, die zur Adsorption sowohl von $\mathrm{CO}_{2}$ als auch von $\mathrm{H}_{2} \mathrm{~S}$ zur Verfügung stehen. Diese Überlegungen sind in Abbildung 37 nochmals in schematischer Form veranschaulicht. 


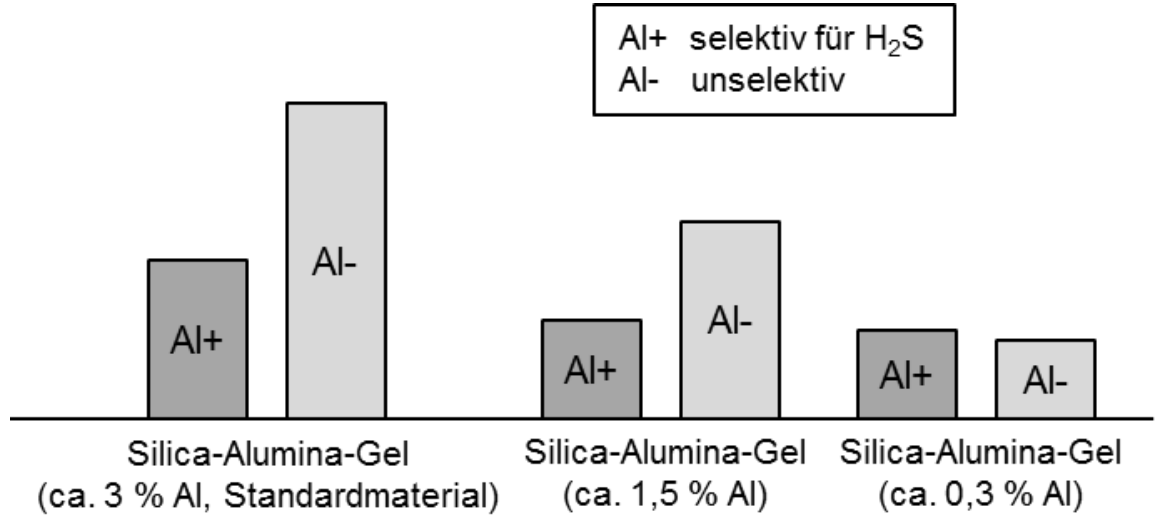

Abbildung 37: Schematische Verteilung verschiedener Aluminiumplätze der Silica-Alumina-Gele

Gemäß dieser Vermutung besitzt das Standardmaterial im Vergleich zu den modifizierten Gelen eine größere Anzahl selektiver aber vor allem eine deutlich größere Anzahl unselektiver Aluminiumplätze. Für die beiden modifizierten Gele wäre die Anzahl der selektiven Aluminiumplätze sehr ähnlich. Die Anzahl unselektiver Aluminiumplätze wäre für beide Gele sehr klein. Mit diesen Annahmen lassen sich die Ergebnisse der binären und der ternären Stoffsysteme konsistent erklären.

Die $\mathrm{CO}_{2}$-Kapazitäten der binären Systeme steigen bei geringeren Aluminiumanteilen im Adsorbens an (vgl. Abbildung 22). Die Oberfläche wird größer, es sind weniger selektive und viel weniger unselektive Aluminiumplätze, dafür jedoch mehr Silanol-Plätze vorhanden. Die immer noch sehr geringen, jedoch im Vergleich zum Standardmaterial leicht gestiegenen Kapazitäten müssen auf van der Waals-Kräfte zurückzuführen sein.

Die $\mathrm{H}_{2} \mathrm{~S}-\mathrm{Kapazitäten} \mathrm{der} \mathrm{binären} \mathrm{Mischungen} \mathrm{sinken} \mathrm{mit} \mathrm{geringerem} \mathrm{Aluminiumgehalt.} \mathrm{Die}$ Anzahl selektiver Al-Plätze ist für beide modifizierte Materialien etwas geringer, liegt aber im selben Bereich.

Für den Fall der ternären Untersuchungen sind die Kapazitätsverluste für $\mathrm{H}_{2} \mathrm{~S}$ relativ zur binären Untersuchung für beide modifizierte Materialien deutlich geringer. Während beim Standardmaterial die Aufnahmefähigkeit für $\mathrm{H}_{2} \mathrm{~S}$ auf Adsorption an selektiven und nicht selektiven Plätzen beruht, haben die modifizierten Gele viel weniger nicht selektive Plätze und das $\mathrm{H}_{2} \mathrm{~S}$ ist vorwiegend auf den selektiven Plätzen gebunden. Die erhöhte $\mathrm{CO}_{2}$ Konzentration hat damit einen deutlich geringeren Einfluss auf die $\mathrm{H}_{2} \mathrm{~S}-$ Kapazität.

Die angestellten Vermutungen ließen sich durch weitere Festkörper-NMR-Untersuchungen der Modifizierten Silica-Alumina-Gele verifizieren. Solche Untersuchungen sind jedoch nicht am Lehrstuhl durchführbar und würden den Rahmen der vorliegenden Untersuchung sprengen. Alternativ könnten Untersuchungen an weiteren modifizierten Silica-Alumina-GelVarianten durchgeführt werden. Von Interesse wären hier insbesondere Materialien mit 
deutlich höherem Al-Gehalt als das Standardmaterial. Diese Fragestellungen werden im Rahmen eines sich an die vorliegende Arbeit anschließenden Promotionsvorhabens untersucht.

\section{Einfluss von $\mathrm{H}_{2} \mathrm{O}$ auf die $\mathrm{H}_{2} \mathrm{~S}$-Adsorption}

Im Vergleich zum Stoffsystem $\left(\mathrm{H}_{2} \mathrm{~S}+\mathrm{CO}_{2}\right.$ in $\left.\mathrm{CH}_{4}\right)$ ist der beobachtete Kapazitätsrückgang bei Anwesenheit von Wasser viel stärker ausgeprägt. Dies ist auf die deutlich höhere Affinität des Wassers zur polaren Silica-Alumina-Gel-Oberfläche zurückzuführen (vgl. Dipolmomente aus Tabelle 16). Für beide ternären Stoffsysteme konnten während der Beladung des Bettes unterschiedlich stark ausgeprägte Verdrängungseffekte von $\mathrm{H}_{2} \mathrm{~S}$-Molekülen infolge adsorbierenden Wassers nachgewiesen werden. Um dies zu verdeutlichen, sind in Abbildung 38 normierte Durchbruchskurven simultan detektierter Signale für $\mathrm{H}_{2} \mathrm{~S}$ und $\mathrm{H}_{2} \mathrm{O}$ einer Untersuchung des Stoffgemisches $\mathrm{H}_{2} \mathrm{~S}+500$ ppm $\mathrm{H}_{2} \mathrm{O}$ in $\mathrm{CH}_{4}$ dargestellt.

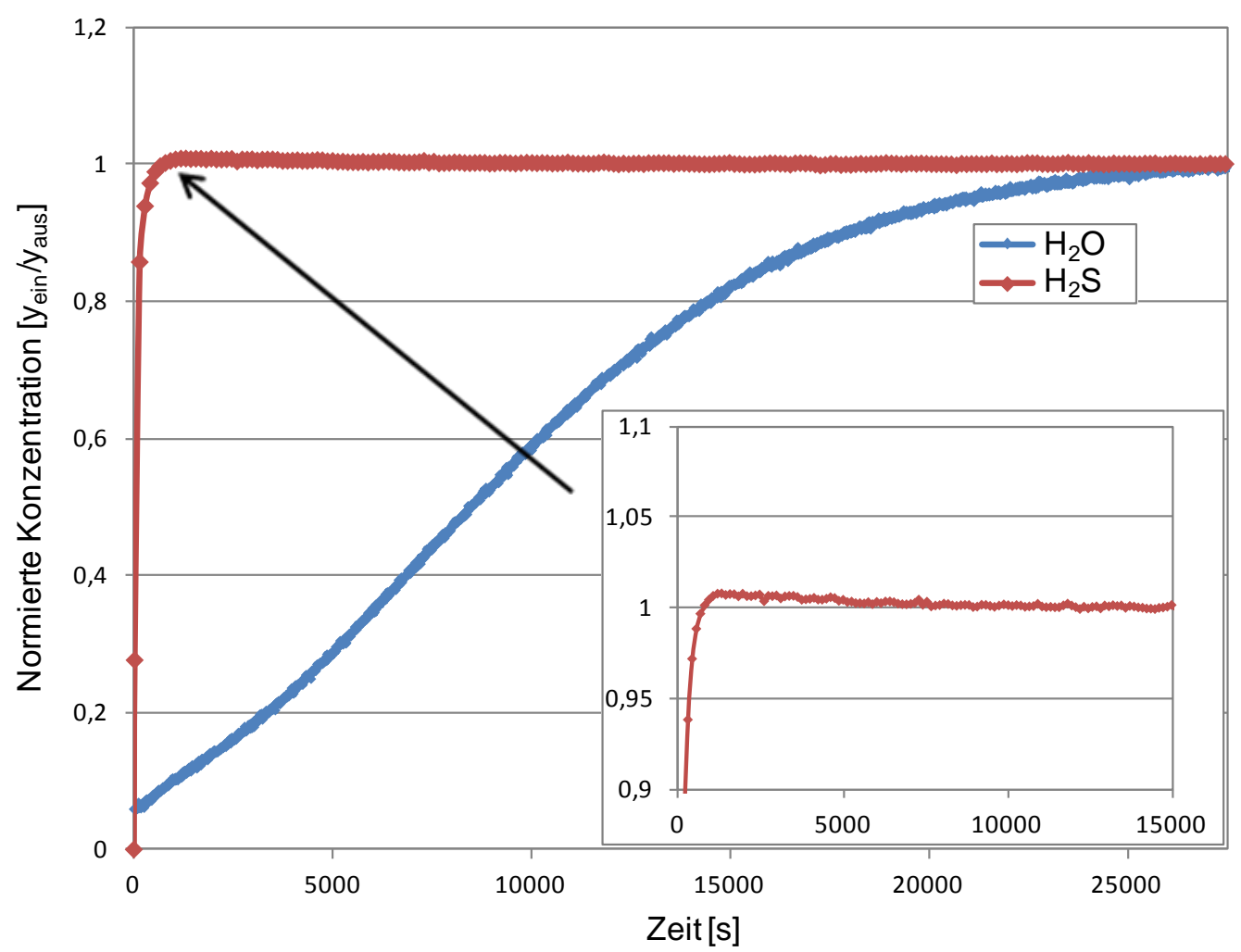

Abbildung 38: Normierte Durchbruchskurven für ein Experiment des ternären Stoffsystems $\mathrm{H}_{2} \mathrm{~S}+$

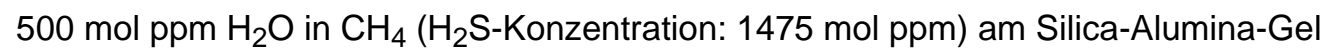

Bei Betrachtung der in der rechten unteren Ecke des Diagramms dargestellten Vergrößerung des $\mathrm{H}_{2} \mathrm{~S}$-Durchbruchsverhaltens zu Beginn des Versuchs lässt sich ein Überschwingen der 
$\mathrm{H}_{2} \mathrm{~S}$-Konzentration über die Feedkonzentration hinaus erkennen. Gebundene $\mathrm{H}_{2} \mathrm{~S}$-Moleküle werden durch adsorbierende $\mathrm{H}_{2} \mathrm{O}$-Moleküle von ihren Adsorptionsplätzen verdrängt und gelangen wieder in die fluide Phase. Nach einer Versuchsdauer von ca. 10000 Sekunden kommt die Verdrängung des $\mathrm{H}_{2} \mathrm{~S}$ zum Erliegen und es stellt sich für die $\mathrm{H}_{2} \mathrm{~S}$-Konzentration ein konstanter Wert ein. Das im weiteren Versuchsverlauf adsorbierende Wasser hat keinen Einfluss mehr auf den Gleichgewichtszustand des Schwefelwasserstoffs.

\subsubsection{Adsorption an 5A Zeolith}

\section{Einfluss von $\mathrm{CO}_{2}$ auf die $\mathrm{H}_{2} \mathrm{~S}$-Adsorption}

Wie für das Silica-Alumina-Gel kommt es auf dem 5A Zeolithen ebenfalls zu einem Kapazitätsverlust, wenn eine weitere Verbindung in der Gasmischung enthalten ist. Der Rückgang in der $\mathrm{H}_{2} \mathrm{~S}$-Beladung für die ternären Stoffsysteme bei unterschiedlichen $\mathrm{CO}_{2^{-}}$ Konzentrationen lässt sich anhand eines Vergleiches normierter Durchbruchskurven mit dem binären Stoffsystem für $\mathrm{H}_{2} \mathrm{~S}$-Feedkonzentrationen im Bereich von 2000 mol ppm veranschaulichen. Abbildung 39 zeigt die entsprechenden Kurven. 


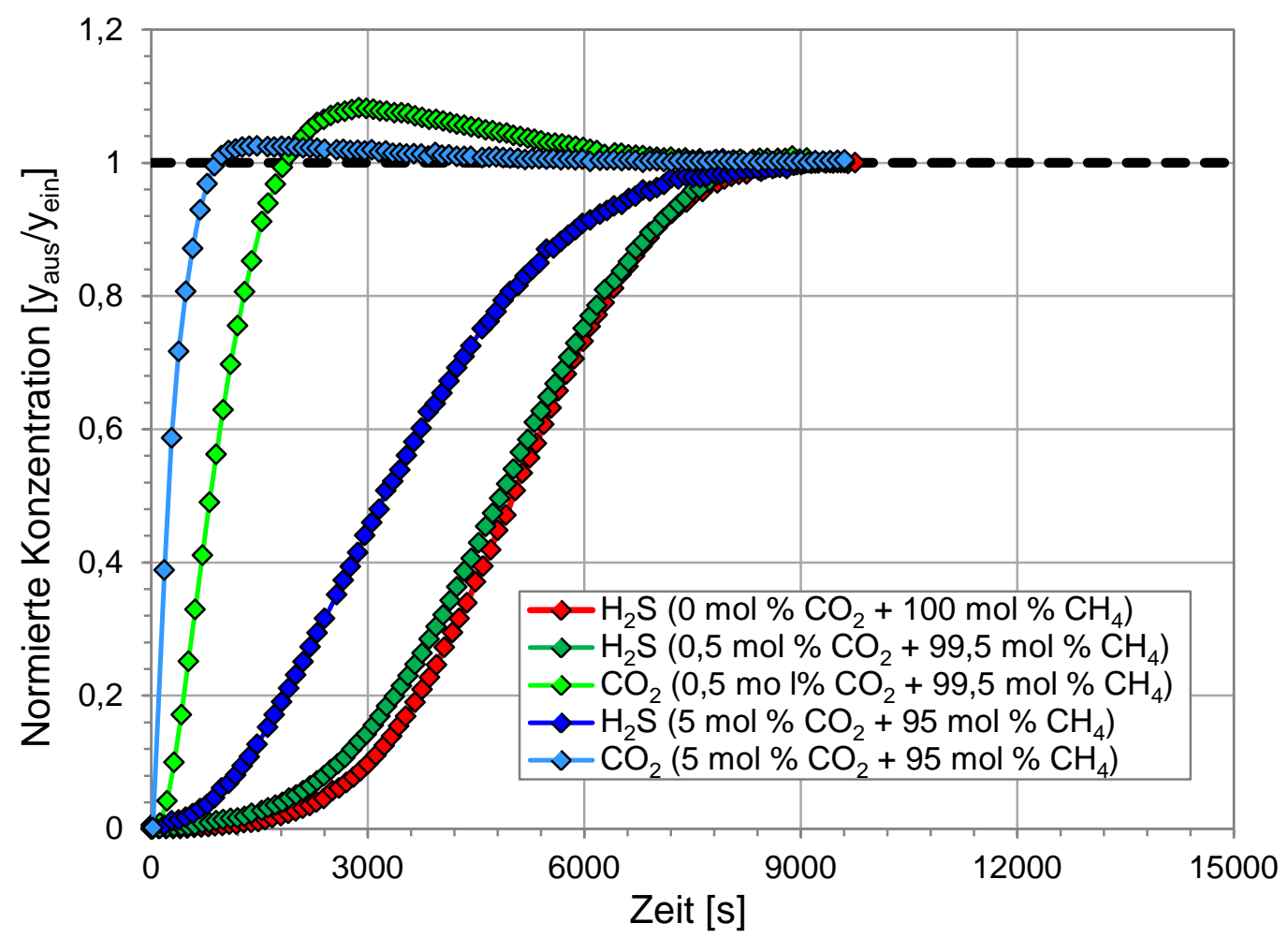

Abbildung 39: Normierte Durchbruchskurven für die Adsorption von $\mathrm{H}_{2} \mathrm{~S}$ und $\mathrm{CO}_{2}$ am Zeolith $5 \mathrm{~A}$ $\left(\mathrm{y}_{\mathrm{H}_{2} \mathrm{~S}} \approx 2000 \mathrm{~mol}-\mathrm{ppm}\right)$; Rote Symbole: $\mathrm{H}_{2} \mathrm{~S}$-Signal für das binäre Stoffsystem. Grüne und blaue Symbole: Simultan detektierte Signale für $\mathrm{H}_{2} \mathrm{~S}$ (dunkle Farben) und $\mathrm{CO}_{2}$ (helle Farben) für die ternären Stoffsysteme

Bei Betrachtung der Konzentrationsfronten erkennt man bei beiden ternären Experimenten eine deutliche Überschreitung der $\mathrm{CO}_{2}$-Feedkonzentration, die typisch für Verdrängungseffekte in Festbetten ist. Die aus der nachlaufenden $\mathrm{H}_{2} \mathrm{~S}$-Front adsorbierenden polaren Schwefelwasserstoffmoleküle sind in der Lage, einen bedeutenden Teil der infolge der vorlaufenden $\mathrm{CO}_{2}$-Front bereits gebundenen unpolaren Kohlendioxidmoleküle von deren Adsorptionsplätzen auf dem polaren Zeolithen zu verdrängen. Die Konzentration erreicht Werte, die über der $\mathrm{CO}_{2}$-Eingangskonzentration liegen.

Die $\mathrm{H}_{2} \mathrm{~S}$-Fronten der ternären Stoffsysteme (dunkel blau und grün) werden vom $\mathrm{CO}_{2}$ beeinflusst. Vor allem im Falle der höheren $\mathrm{CO}_{2}$-Konzentration in der Gasmischung ergibt sich für die $\mathrm{H}_{2} \mathrm{~S}$-Durchbruchskurve im Vergleich zum binären System eine unterschiedliche Form. Der Durchbruch des $\mathrm{H}_{2} \mathrm{~S}$ beginnt früher und die Durchbruchskurve weist eine unterschiedliche Steigung auf. Im Bereich der Gleichgewichtseinstellung verläuft die Front deutlich flacher. $\mathrm{H}_{2} \mathrm{~S}$ kann nur noch einen Teil der adsorbierten $\mathrm{CO}_{2}$ Moleküle verdrängen. Ein Großteil der Adsorptionsplätze bleibt vom $\mathrm{CO}_{2}$ besetzt. Die $\mathrm{H}_{2} \mathrm{~S}$-Front in Anwesenheit der 
geringen $\mathrm{CO}_{2}$-Konzentration erfährt im Vergleich zur Kurve des binären Systems nur eine geringe Beeinflussung. Der Durchbruch findet zu einem etwas früheren Zeitpunkt statt. Im Bereich der Gleichgewichtseinstellung verlaufen die Kurven deckungsgleich.

Wie bei dem zuvor untersuchten Silica-Alumina-Gel wurde bei den Experimenten mit dem 5A-Molsieb weder eine COS- noch eine $\mathrm{H}_{2} \mathrm{O}$-Bildung gemäß Reaktionsgleichung 4.1 nachgewiesen. Unter den gegebenen Bedingungen $\left(\mathrm{T}=25^{\circ} \mathrm{C}, \mathrm{p}=1,3\right.$ bar und $\mathrm{H}_{2} \mathrm{O}$-Gehalt des Trägergases $\sim 0$ ) ist daher zu konstatieren, dass keine Reaktion zwischen dem Schwefelwasserstoff und dem Kohlendioxid stattfindet.

\section{Einfluss von $\mathrm{H}_{2} \mathrm{O}$ auf die $\mathrm{H}_{2} \mathrm{~S}$-Adsorption}

Auch in Anwesenheit von Wasser kommt es zu einer deutlichen Verminderung der $\mathrm{H}_{2} \mathrm{~S}$ Kapazitäten auf dem Zeolithen. Für beide Messreihen mit unterschiedlichem Wassergehalt war zu beobachten, dass adsorbierende $\mathrm{H}_{2} \mathrm{O}$ Moleküle einen Großteil gebundener $\mathrm{H}_{2} \mathrm{~S}$ Moleküle wieder aus dem Porensystem des Zeolithen verdrängen. Im Gegensatz zum Stoffsystem $\mathrm{H}_{2} \mathrm{~S}+\mathrm{CO}_{2}$ in $\mathrm{CH}_{4}$ ist $\mathrm{H}_{2} \mathrm{~S}$ bei diesem Stoffsystem das Adsorptiv mit der geringeren Affinität zur Zeolithoberfläche. Aus diesem Grund läuft nun die $\mathrm{H}_{2} \mathrm{~S}$-Front schneller durch das Bett als die $\mathrm{H}_{2} \mathrm{O}$-Front. Prinzipiell sind die gleichen Phänomene wie für das Stoffsystem $\mathrm{H}_{2} \mathrm{~S}+\mathrm{CO}_{2}$ in $\mathrm{CH}_{4}$ zu beobachten. Daher soll an dieser Stelle auf weitere Darstellungen verzichtet werden.

\subsubsection{Vergleich der Adsorbentien}

Betrachtet man die für beide Adsorbentien vermessenen ternären Stoffsysteme $\left(\mathrm{H}_{2} \mathrm{~S}+\mathrm{CO}_{2}\right.$ in $\mathrm{CH}_{4}, \mathrm{H}_{2} \mathrm{~S}+\mathrm{H}_{2} \mathrm{O}$ in $\mathrm{CH}_{4}$ ), zeigt sich ein vergleichbares Bild. Für alle untersuchten Stoffsysteme führt eine zweite Komponente in der Gasmischung $\left(\mathrm{CO}_{2}\right.$ oder $\left.\mathrm{H}_{2} \mathrm{O}\right)$ zu einem Kapazitätsverlust für $\mathrm{H}_{2} \mathrm{~S}$. Einer Konzentrationserhöhung dieser Komponente folgt jeweils auch ein zusätzlicher $\mathrm{H}_{2} \mathrm{~S}$-Kapazitätsverlust. Die Rolle des $\mathrm{H}_{2} \mathrm{~S}$ bei dieser Mehrkomponentenadsorption bedarf jedoch einer differenzierteren Betrachtung.

$\mathrm{H}_{2} \mathrm{~S}+\mathrm{CO}_{2}$ in $\mathrm{CH}_{4}$. Bei diesem Stoffsystem ist $\mathrm{H}_{2} \mathrm{~S}$ auf Grund seiner Polarität für beide Adsorbentien das stärker bindende Adsorptiv mit der höheren Affinität zum Adsorbens. Dies wurde durch die binären Untersuchungen $\left(\mathrm{H}_{2} \mathrm{~S}\right.$ in $\mathrm{CH}_{4}, \mathrm{CO}_{2}$ in $\left.\mathrm{CH}_{4}\right)$ gezeigt. $\mathrm{CO}_{2}$ adsorbiert bedingt durch seine schnellere Kinetik und die deutlich geringeren Kapazitäten zuerst. Es 
kommt zu einer Vorbeladung der Oberfläche. Adsorbierenden $\mathrm{H}_{2} \mathrm{~S}$-Molekülen stehen weniger Adsorptionsplätze zur Verfügung. Im Falle des Zeolithen ist $\mathrm{H}_{2} \mathrm{~S}$ in der Lage, bereits von $\mathrm{CO}_{2}$-Molekülen besetzte Plätze zurück zu gewinnen.

$\mathbf{H}_{2} \mathbf{S}+\mathbf{H}_{2} \mathrm{O}$ in $\mathbf{C H}_{4}$. Bei diesen Untersuchungen ist $\mathrm{H}_{2} \mathrm{~S}$ das schwächer bindende Adsorptiv mit der deutlich geringeren Affinität zum Adsorbens. Im Falle beider Adsorbentien kommt es zu einer Verdrängung von $\mathrm{H}_{2} \mathrm{~S}$-Molekülen.

Ein Vergleich der Ergebnisse der Untersuchungen der binären Systeme zeigt, dass Wasser auf beiden Adsorbentien um ein Vielfaches besser adsorbiert als $\mathrm{CO}_{2}$. Aus diesem Grund sind die $\mathrm{H}_{2} \mathrm{~S}$-Kapazitätsverluste für die Untersuchungen der ternären Gasgemische im Falle von Wasser bereits bei deutlich geringeren Konzentrationen $\left(500 \mathrm{~mol}\right.$ ppm $\mathrm{H}_{2} \mathrm{O}<=>5000$ mol ppm $\mathrm{CO}_{2}, 1000 \mathrm{~mol}$ ppm $\mathrm{H}_{2} \mathrm{O}<=>50000$ mol ppm $\mathrm{CO}_{2}$ ) wesentlich stärker ausgeprägt als für konkurrierendes $\mathrm{CO}_{2}$.

\subsubsection{Vorhersage von Mehrkomponentenadsorptionsgleichgewichten}

Die Untersuchungen zu den ternären Stoffsystemen $\left(\mathrm{H}_{2} \mathrm{~S}+\mathrm{CO}_{2}\right.$ in $\left.\mathrm{CH}_{4}\right)$ an den beiden Standard-Adsorbentien bieten sich für einen Vergleich mit den in Abschnitt 2.1.1 eingeführten Modellen zur Vorhersage von Mehrkomponentenadsorptionsgleichgewichten an. Als Berechnungsgrundlage dieser Modelle dienen lediglich die Gleichgewichte der jeweiligen binären Stoffsysteme $\left(\mathrm{H}_{2} \mathrm{~S}\right.$ in $\mathrm{CH}_{4}, \mathrm{CO}_{2}$ in $\left.\mathrm{CH}_{4}\right)$. Ein Vergleich der Modelle mit den experimentell ermittelten Gleichgewichten ermöglicht die Diskussion der Vorhersagequalität.

Die im Rahmen der Arbeit verwendete Untersuchungsmethode ermöglicht die simultane Vermessung der Durchbruchskurven der beteiligten Adsorptive und mit Hilfe der Massenbilanzen die Bestimmung der jeweiligen Beladung. Im Gegensatz zu Experimenten mit gravimetrischen oder volumetrischen Versuchsapparaturen lässt sich damit für Systeme mit mehreren Adsorptiven die Zusammensetzung der Adsorbatphase bestimmen. Abbildung 40 soll dies anhand der schematisch dargestellten Durchbruchskurven verdeutlichen. 


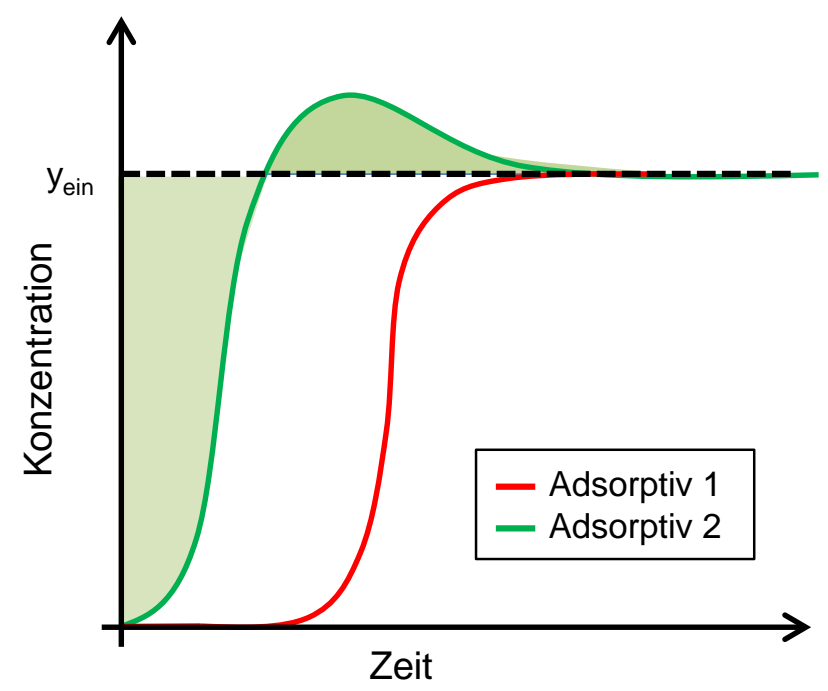

Abbildung 40: Schematische Darstellung simultan detektierter Durchbruchskurven für ein Stoffsystem mit zwei Adsorptiven

Hierbei wird die Adsorbensbeladung für Adsorptiv 1 gemäß der in Abschnitt XY erläuterten Bilanzierung durchgeführt. Gleichzeitig lässt sich jedoch auch die Beladung für Adsorptiv 2 ermitteln:

Das schwächere Adsorptiv 2 wird teilweise durch Adsorptiv 1, welches eine höhere Affinität zum Adsorbens aufweist, wieder aus dem Porensystem verdrängt. Dies zeigt sich im Überschwingen der Konzentration über die Feedkonzentration $\mathrm{y}_{\text {ein. }}$. In der Folge streben beide Adsorptive ein Gleichgewicht an. Rechnerisch lässt sich die auf dem Adsorbens verbleibende Stoffmenge durch Subtraktion der grün schraffierten Flächen ermitteln. Bei Vernachlässigung einer Trägergasadsorption (vgl. Abschnitt 3.2.3 zur Versuchsdurchführung) erhält man somit Kenntnis über die Zusammensetzung der adsorbierten Phase.

\section{Stoffsysteme mit Langmuir-Verhalten}

Im Folgenden soll ein Vergleich der experimentell ermittelten Beladungen für das ternäre Stoffsystem $\left(\mathrm{H}_{2} \mathrm{~S}+\mathrm{CO}_{2}\right.$ in $\left.\mathrm{CH}_{4}\right)$ am $5 \mathrm{~A}$ Zeolithen mit den Modellen nach Markham und Benton und der IAS-Theorie nach Myers und Prausnitz durchgeführt werden. Anhand Abbildung 41 sollen zunächst die experimentellen Ergebnisse erläutert werden. 


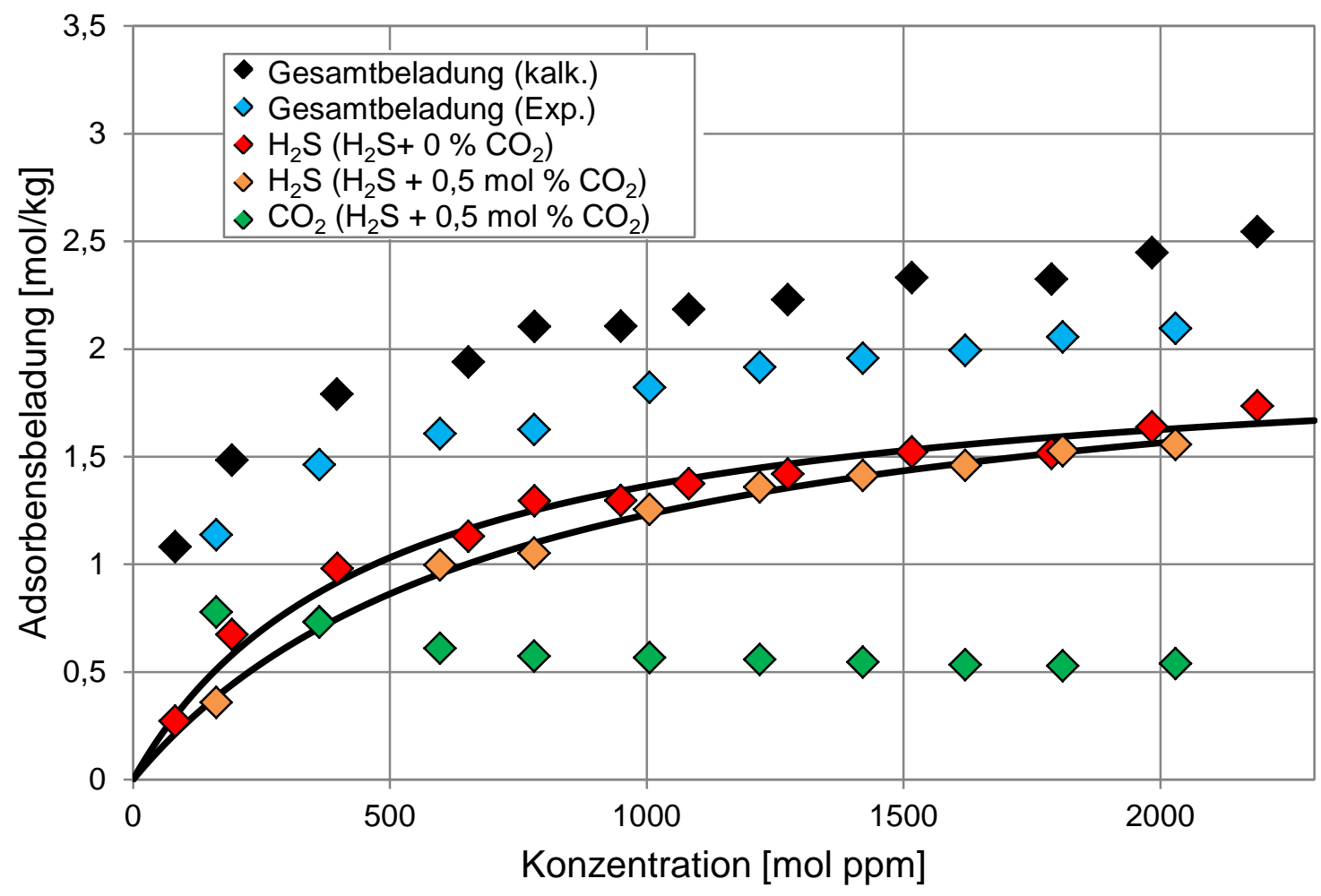

Abbildung 41: Beladungen für das ternäre Stoffsystem $\left(\mathrm{H}_{2} \mathrm{~S}+0,5 \mathrm{~mol} \% \mathrm{CO}_{2}\right.$ in $\mathrm{CH}_{4} / 5 \mathrm{~A}$ Zeolith)

Die Abbildung zeigt die $\mathrm{H}_{2} \mathrm{~S}$-Isotherme für den binären Fall (rote Messwerte). Ist eine Konzentration von 0,5 mol \% Kohlendioxid in der Gasmischung enthalten, ergibt sich gemäß der Abbildung der bereits diskutierte, geringe Abfall in der $\mathrm{H}_{2} \mathrm{~S}$-Kapazität (orange Messwerte). Gleichzeitig lassen sich gemäß dem in Abbildung 40 dargestellten Schema die $\mathrm{CO}_{2^{-}}$ Kapazitäten ermitteln und in Abhängigkeit der $\mathrm{H}_{2} \mathrm{~S}$-Konzentration darstellen (grüne Messwerte).

Die maximale Gesamtbeladung (schwarze Punkte) kann als hypothetische Obergrenze durch eine Addition der Einzelkapazitäten aus den binären Messreihen kalkuliert werden. Dabei wird angenommen, dass keine Verdrängungseffekte auftreten und jedem Adsorptiv unendlich viele Adsorptionsplätze zur Verfügung stehen.

Für die Adsorption nach der Langmuir-Theorie ist dieser Grenzwert nicht zu erreichen, da die

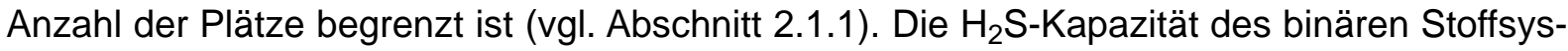
tems (rote Punkte) stellt die hypothetische Untergrenze für die Gesamtbeladung dar, die erreicht würde, wenn das $\mathrm{CO}_{2}$ im ternären System nur auf solchen Plätzen adsorbieren würde, die im binären System noch von $\mathrm{H}_{2} \mathrm{~S}$ besetzt sind.

Die experimentelle Gesamtbeladung (blaue Punkt) im ternären System lässt sich durch Addition der experimentell bestimmten Kapazitäten der beiden Adsorptive im ternären Sys- 
tem bestimmen (orange und grüne Messwerte). Die experimentelle Gesamtbeladung sollte zwischen den hypothetischen Grenzen liegen.

Die Abbildung zeigt, dass dies zutrifft. Die Lage unterhalb der schwarzen Punkte erscheint plausibel, da die Experimente mit dem ternären Stoffsystem $\left(\mathrm{H}_{2} \mathrm{~S}+\mathrm{CO}_{2}\right.$ in $\left.\mathrm{CH}_{4}\right)$ eine ausgeprägte Verdrängung des schwächer adsorbierenden $\mathrm{CO}_{2}$ gezeigt haben. Damit korrespondieren auch die ermittelten $\mathrm{CO}_{2}$-Kapazitäten (grüne Punkte). Diese nehmen zu höheren $\mathrm{H}_{2} \mathrm{~S}$-Konzentrationen ab und laufen ähnlich wie die $\mathrm{H}_{2} \mathrm{~S}$-Isotherme in einen Plateauwert. Je höher die $\mathrm{H}_{2} \mathrm{~S}$-Konzentration in der Gasmischung ist, desto ausgeprägter ist auch die Verdrängung des $\mathrm{CO}_{2}$ und entsprechend geringer die ermittelten Kapazitäten. Die Lage oberhalb der roten Punkte belegt zusammen mit der geringfügig verminderten $\mathrm{H}_{2} \mathrm{~S}$ Kapazität im ternären System, dass das $\mathrm{CO}_{2}$ zum einen Teil auf Plätzen adsorbiert, die im binären System von $\mathrm{H}_{2} \mathrm{~S}$ belegt waren und zum anderen Teil auf Plätzen, die im binären System unbesetzt waren.

\section{IAS-Theorie}

Abbildung 42 zeigt die Ergebnisse der Gleichgewichtsvorhersage mit Hilfe der IAS-Theorie. Zum Vergleich sind die zuvor gezeigten, experimentell bestimmten Gleichgewichte mit dargestellt (graue Punkte und Linien). 


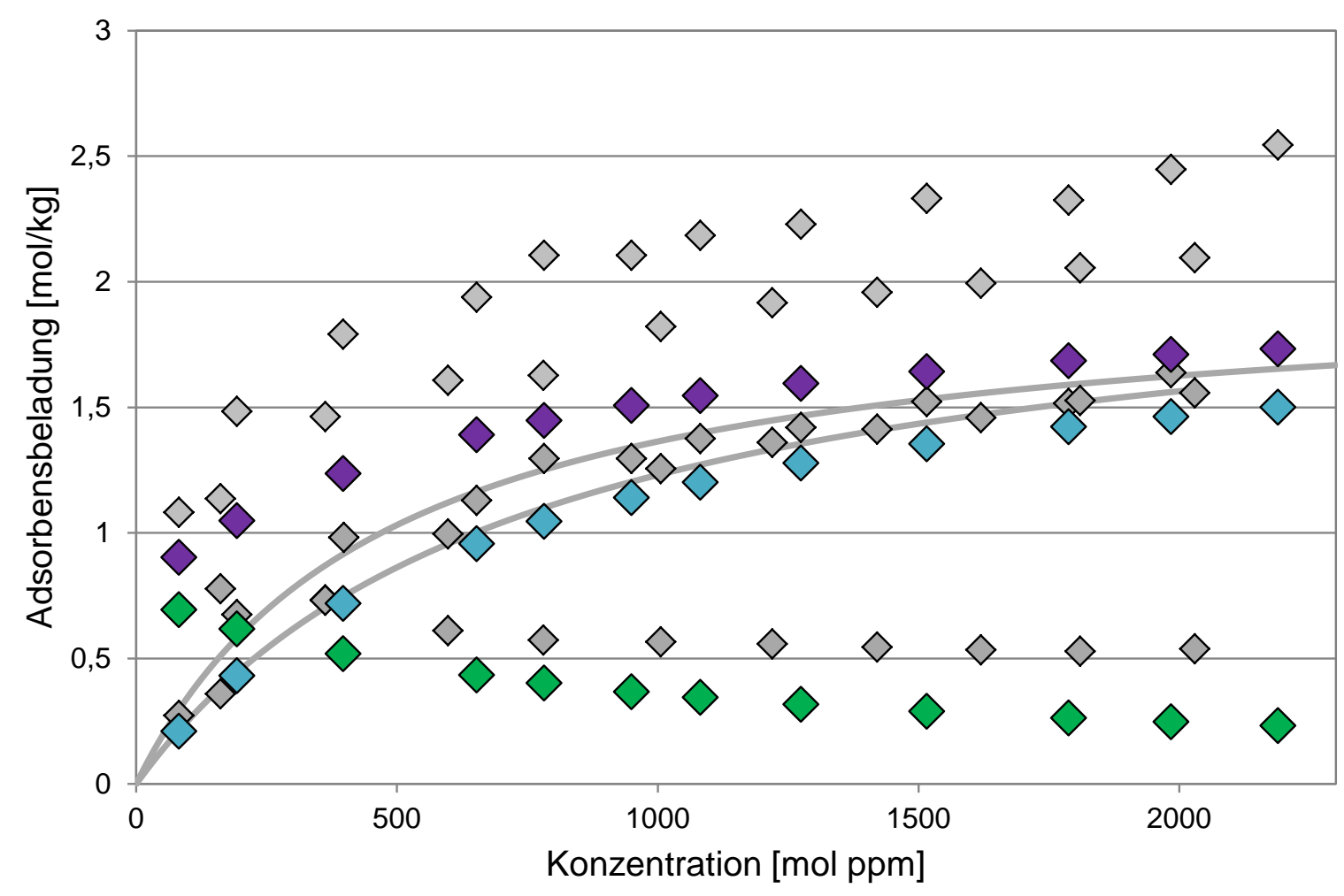

Abbildung 42: Berechnete Adsorptionsgleichgewichte nach IAST (farbige Symbole). Blaue Symbole: $\mathrm{H}_{2} \mathrm{~S}$-Beladungen, grüne Symbole: $\mathrm{CO}_{2}$-Beladungen in Abhängigkeit der jeweiligen $\mathrm{H}_{2} \mathrm{~S}$-Konzentration, lila Symbole: Gesamtbeladung

Die IAST-Berechnungen zeigen zwei eindeutige Ergebnisse. Die Vorhersage der $\mathrm{H}_{2} \mathrm{~S}$ Kapazitäten (hellblaue Punkte) geschieht in sehr guter Näherung zu den experimentellen Werten. Im unteren Konzentrationsbereich (bis 500 mol ppm) entsprechen sich Theorie und Experiment fast exakt. Im höheren Konzentrationsbereich, mit dem Verlauf in das LangmuirPlateau, ist eine größere Abweichung zu beobachten. Trotzdem ist in diesem Bereich die Vorhersage von guter Qualität. Im Bereich einer $\mathrm{H}_{2}$ S-Konzentration von 2000 mol ppm beträgt die Abweichung zwischen Experiment und dem IAST-Modell ca. 6,3 \%.

Bei Betrachtung der vorhergesagten $\mathrm{CO}_{2}$-Beladungen in Abhängigkeit der $\mathrm{H}_{2} \mathrm{~S}$ Konzentration weichen Modell und Experiment deutlich voneinander ab. Das Modell unterschätzt die $\mathrm{CO}_{2}$-Beladungen im Vergleich $\mathrm{zu}$ den experimentell ermittelten Werten. Dementsprechend liegt auch die Gesamtbeladung nach IAST deutlich unter den experimentellen Werten. Allerdings liegen die Gesamtbeladungen nach IAST über der $\mathrm{H}_{2} \mathrm{~S}$-Kapazität der binären Messung. Dies ist konsistent mit den Experimenten. 


\section{Vergleich der IAS-Theorie mit der erweiterten Langmuir-Gleichung nach Mark- ham und Benton}

Abbildung 43 zeigt einen Vergleich der IAS-Theorie mit der erweiterten Langmuir-Gleichung nach Markham und Benton.

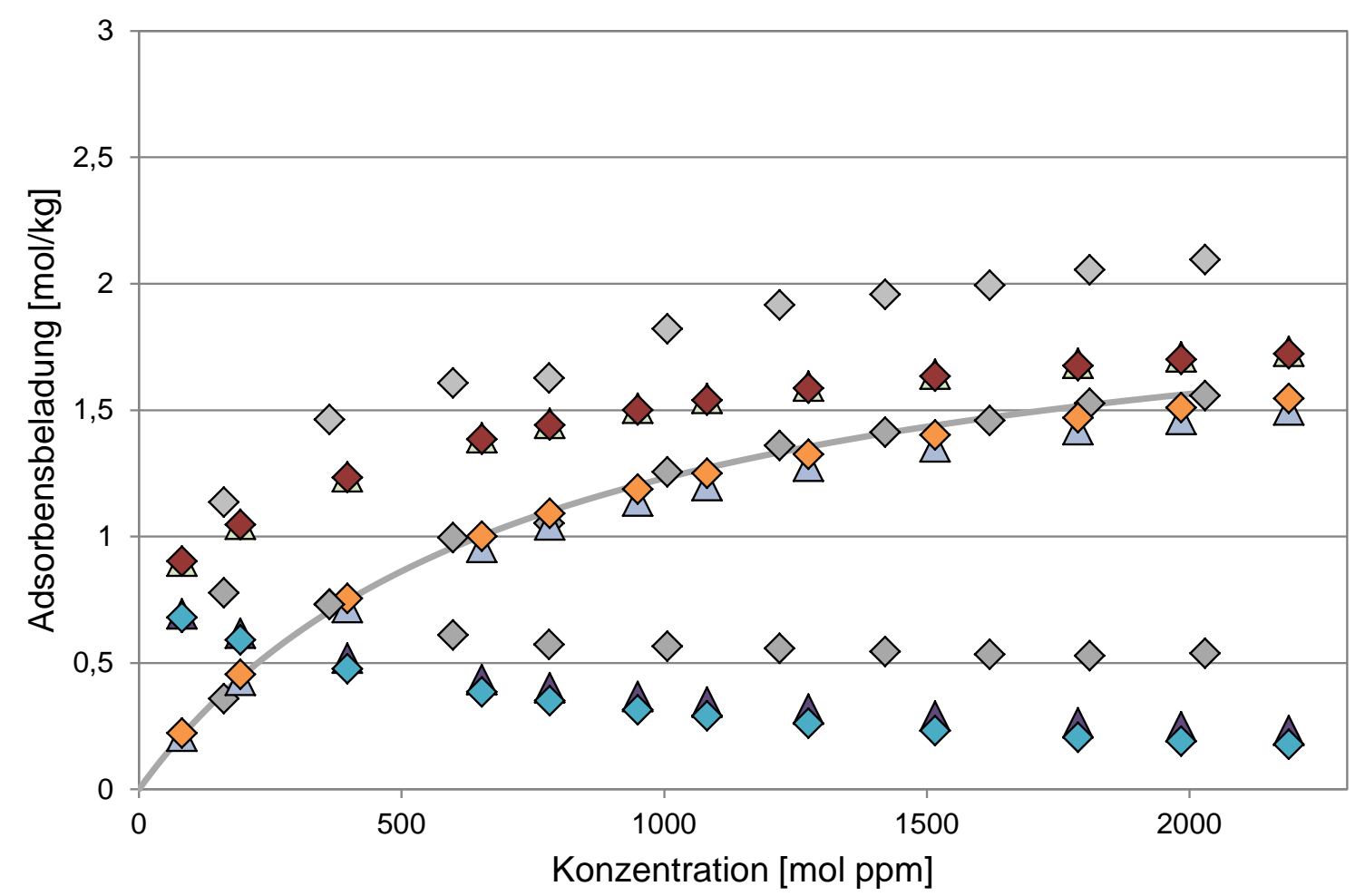

Abbildung 43: Vergleich der IAS-Theorie mit der erweiterten Langmuir-Gleichung nach Markham und Benton (Markham und Benton: farbige Dreiecke; IAS-Theorie: farbige Rauten)

Die Vorhersage der $\mathrm{H}_{2} \mathrm{~S}$-Kapazitäten mit Hilfe der erweiterten Langmuir-Gleichung nach Markham und Benton für das ternäre Stoffsystem $\left(\mathrm{H}_{2} \mathrm{~S}+0,5 \mathrm{~mol} \% \mathrm{CO}_{2}\right.$ in $\left.\mathrm{CH}_{4}\right)$ gelingt mit noch größerer Genauigkeit (orange Dreiecke). Modell und Experiment gleichen sich stark. Im Bereich einer $\mathrm{H}_{2}$ S-Konzentration von 2000 mol ppm beträgt die Abweichung zwischen Experiment und dem Modell ca. 3,3\%. Die experimentellen $\mathrm{CO}_{2}$-Kapazitäten werden wie bei der IAS-Theorie (Rauten) deutlich unterschätzt. Es werden noch geringere Kapazitätswerte vorhergesagt. Bei der Vorhersage der gesamt adsorbierten Stoffmenge entsprechen sich beide Modelle.

Zusammenfassend ist zu konstatieren, dass beide Modelle die Adsorption des ternären Stoffsystems mit 0,5 mol \% $\mathrm{CO}_{2}$ in der Gasmischung am 5A Zeolithen mit sehr guter Genauigkeit vorhersagen können. Die Beladungen des Adsorptivs, dessen Konzentration variiert 
wird $\left(\mathrm{H}_{2} \mathrm{~S}\right)$, werden dabei mit beiden Modellen besser vorhergesagt, als die Beladungen des Adsorptivs, welches mit konstanter Konzentration in der Gasmischung vorliegt $\left(\mathrm{CO}_{2}\right)$.

Werden die Modelle auf das im Rahmen der Arbeit untersuchte ternäre Stoffsystem mit der hohen $\mathrm{CO}_{2}$-Konzentration angewendet $\left(\mathrm{H}_{2} \mathrm{~S}+5 \mathrm{~mol} \% \mathrm{CO}_{2}\right.$ in $\left.95 \mathrm{~mol} \% \mathrm{CH}_{4}\right)$, ergeben sich deutlich größere Abweichungen zwischen den Modellen und den experimentellen Befunden. Abbildung 44 zeigt die entsprechenden Ergebnisse für die $\mathrm{H}_{2} \mathrm{~S}$-Beladungen dieses Stoffsystems. Zum Vergleich ist die $\mathrm{H}_{2} \mathrm{~S}$-Isotherme des binären Systems mit aufgeführt.

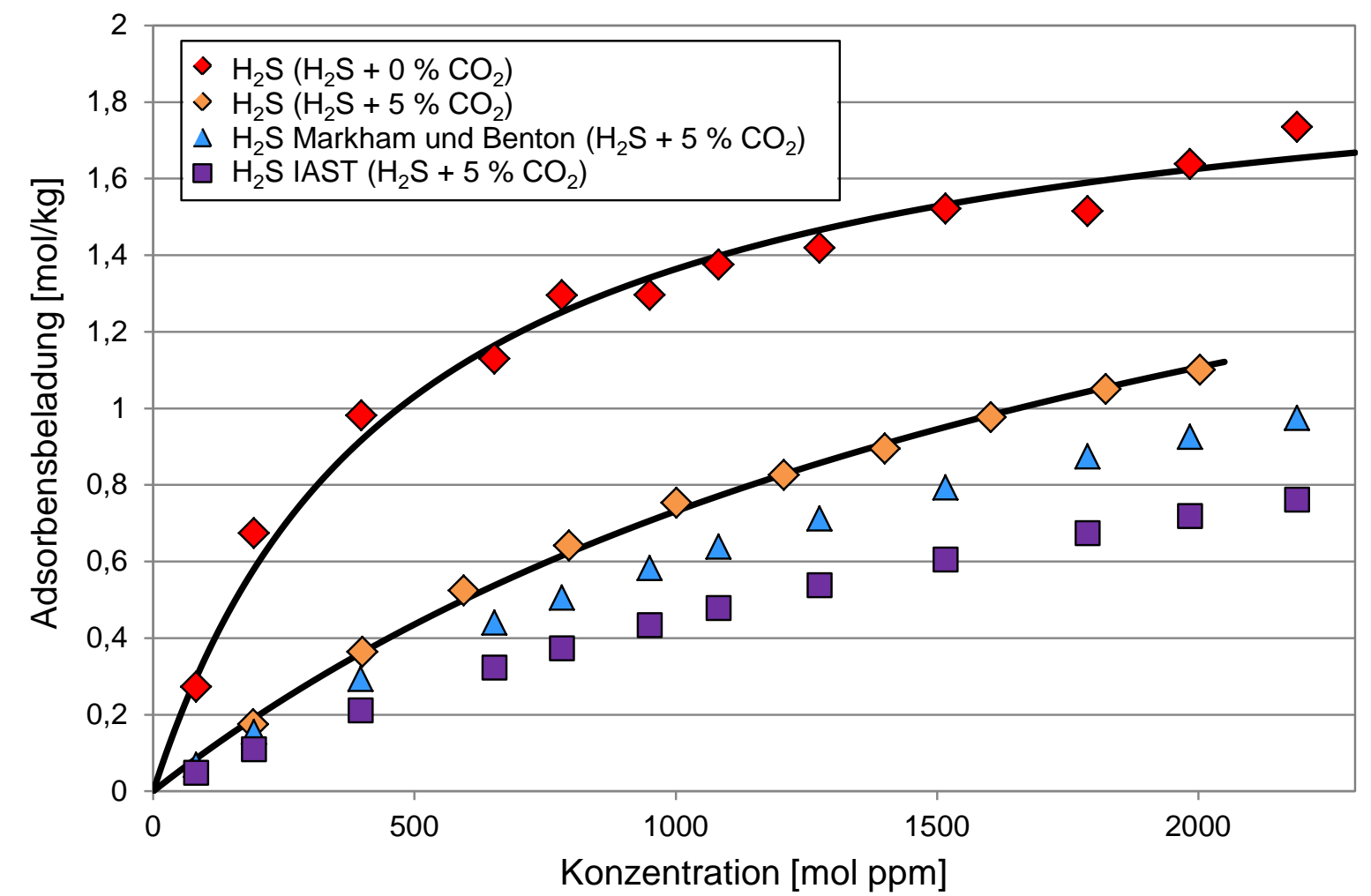

Abbildung 44: Vergleich der experimentellen Befunde mit den Vorhersagemodellen für das Stoffsystem $\mathrm{H}_{2} \mathrm{~S}+5 \mathrm{~mol} \% \mathrm{CO}_{2}+95 \mathrm{~mol} \% \mathrm{CH}_{4} / 5 \mathrm{~A}$ Zeolith

Wiederum ist die Vorhersage mittels erweiterter Langmuir-Isotherme nach Markham und Benton (hellblaue Dreiecke) deutlich näher an den experimentell nachgewiesenen $\mathrm{H}_{2} \mathrm{~S}$ Beladungen als die ermittelten Werte der IAS-Theorie (lila Quadrate). Im Bereich einer $\mathrm{H}_{2} \mathrm{~S}$ Konzentration von 2000 mol ppm ergibt sich demnach eine Abweichung von ca. $16 \%$ für die erweiterte Langmuir-Isotherme und ca. 35 \% für die IAS-Theorie bezogen auf die experimentellen Werte.

Ein Grund für die starke Abweichung zwischen den experimentellen Beladungen und den Modellen, insbesondere der IAS-Theorie, kann anhand der jeweiligen Isothermen der binären Systeme erläutert werden. Abbildung 45 zeigt diese Isothermen. Zur besseren 
Veranschaulichung sind die auf der X-Achse abgetragenen Adsorptivkonzentrationen logarithmisch dargestellt.

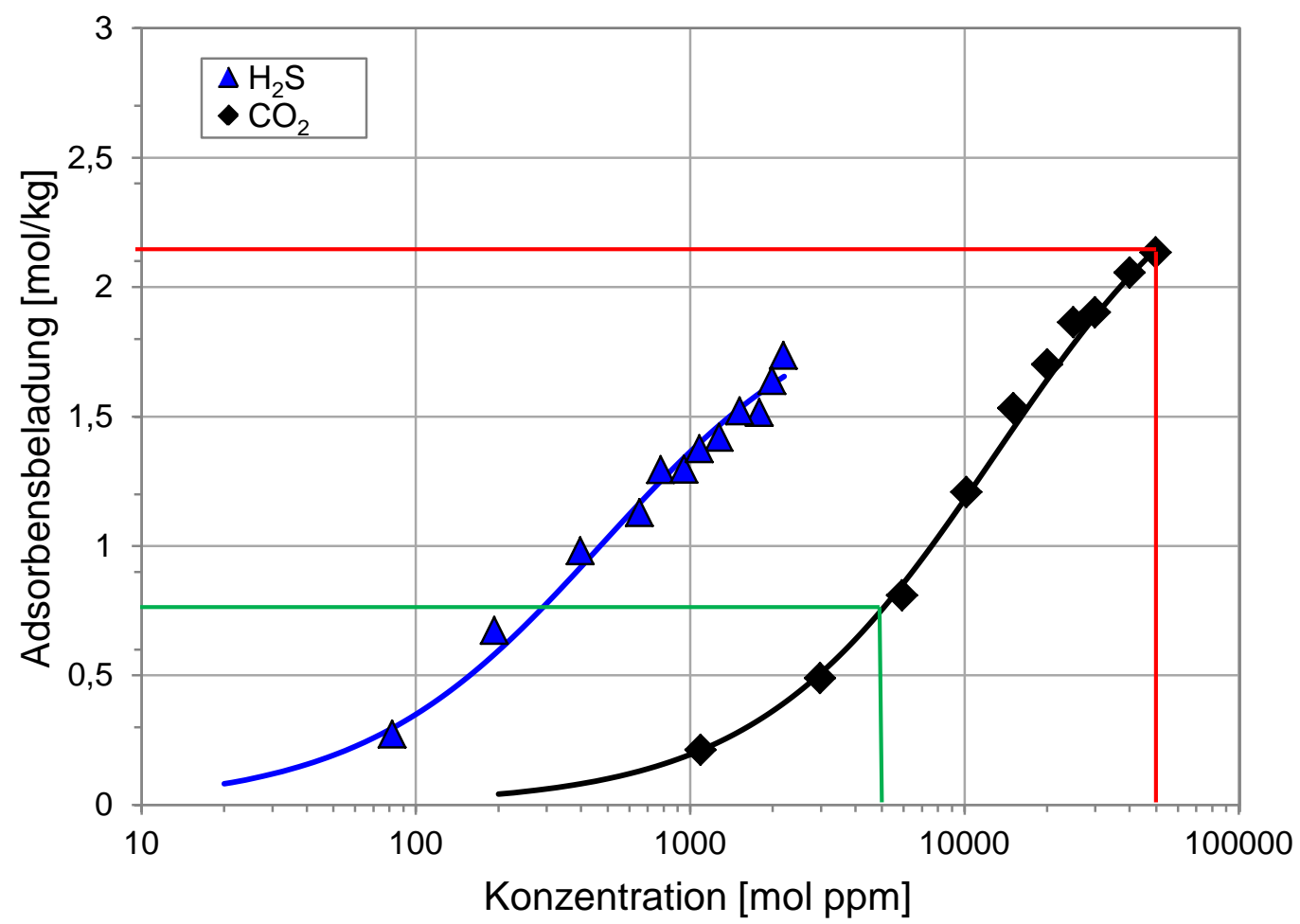

Abbildung 45: Isothermen für die Adsorption von $\mathrm{H}_{2} \mathrm{~S}$ aus $\mathrm{CH}_{4}$ und $\mathrm{CO}_{2}$ aus $\mathrm{CH}_{4}$ an $5 \mathrm{~A}$ Zeolith

Die IAS-Theorie setzt voraus, dass der Spreizdruck, der durch die Adsorption der reinen Komponenten (Adsorption aus einem Trägergas) auf dem Adsorbens hervorgerufen wird, demjenigen Spreizdruck entspricht, der durch die Adsorption der Komponenten in der Mischung entsteht. Der Spreizdruck der Mischung ist darüber hinaus gleich dem Spreizdruck jeder der beiden Komponenten in der Mischung.

Im Falle des ternären Stoffsystems mit $5 \mathrm{~mol} \% \mathrm{CO}_{2}$ in der Gasmischung ist die adsorbierte Menge an $\mathrm{CO}_{2}$ deutlich größer als die adsorbierte Menge an $\mathrm{H}_{2} \mathrm{~S}$ (siehe rote Markierung in Abbildung 45). Zudem ist der Partialdruck in der Gasphase viel höher als der Partialdruckbereich des $\mathrm{H}_{2} \mathrm{~S}$. Dadurch werden bei diesem System für $\mathrm{H}_{2} \mathrm{~S}$ hypothetische Dampfdrücke der Adsorbatphase bestimmt, die deutlich über den Partialdrücken der binären Messungen liegen. Bei der Lösung des Gleichungssystems werden die hypothetischen Dampfdrücke der Komponenten in der Adsorbatphase aus einer Integration ermittelt, bei der die angepassten Parameter $b$ und $X_{\text {mon }}$ der Einzelisothermen verwendet werden. Diese Parameter gelten jedoch lediglich im experimentell untersuchten Partialdruckbereich und müssen demnach extrapoliert werden. Daraus können sich Abweichungen ergeben. 
Für das ternäre Stoffsystem mit der geringen $\mathrm{CO}_{2}$-Konzentration in der Gasmischung besteht dieses Problem nicht (siehe grüne Markierung in Abbildung 45). Die Kapazitäten der binären Systeme für $\mathrm{H}_{2} \mathrm{~S}$ und $\mathrm{CO}_{2}$ liegen im selben Bereich. Auch die Partialdrücke liegen nicht so sehr auseinander. Dadurch lassen sich hypothetische Dampfdrücke im Bereich der experimentellen Partialdrücke finden. Hier ist die Gültigkeit der binären IsothermenParameter gesichert und dementsprechend auch die Vorhersage der $\mathrm{H}_{2} \mathrm{~S}$-Kapazitäten des ternären Gemisches besser.

Ein konzeptioneller Grund für die Abweichung der Vorhersage nach der IAS-Theorie könnte die Annahme einer homogenen Oberfläche sein. Es wäre möglich, dass Bindungsplätze vorhanden sind, die selektiv sind (siehe Abschnitt 4.2.1). Die IAS-Theorie vernachlässigt derartige Selektivitäten.

Ein weiterer Grund für Abweichungen können Wechselwirkungen der Moleküle in der adsorbierten Phase sein. Die IAS-Theorie berücksichtigt derartige Wechselwirkungen nicht. Da es im Vergleich zum ternären Stoffsystem mit der geringeren $\mathrm{CO}_{2}$-Konzentration in der Gasmischung $\mathrm{zu}$ deutlich höheren $\mathrm{CO}_{2}$-Beladungen kommt, könnte es bei der hohen $\mathrm{CO}_{2}$ Konzentration vermehrt $\mathrm{zu}$ Wechselwirkungen zwischen adsorbierten $\mathrm{CO}_{2^{-}}$und $\mathrm{H}_{2} \mathrm{~S}$ Molekülen kommen.

\section{Stoffs ys teme mit Henry-Verhalten}

Für die ternären Stoffsysteme auf dem Silica-Alumina-Gel können ebenfalls Vorhersagen mit Hilfe der IAS-Theorie angestellt werden. Da jedoch beide Einzelisothermen (bestimmt aus binärer Mischung) durch eine Henry-Isotherme beschrieben werden können, ergibt sich ein Spezialfall.

Wie in Abschnitt 2.1.1 erläutert, unterliegt die Henry-Isotherme der Annahme, dass es unendlich viele Adsorptionsplätze gibt, die von Adsorptivmolekülen besetzt werden können. Weiterhin werden die vorhandenen Plätze als energetisch gleichwertig betrachtet. Unter diesen Voraussetzungen beeinflussen sich die Komponenten bei der Adsorption nicht. Für Henry-Isothermen besagt die IAST damit, dass die Isothermen beider Komponenten im binären und im ternären Stoffsystem gleich sind. Die adsorbierte Gesamtstoffmenge ergibt sich durch Addition der Einzelstoffmengen.

Der experimentell belegte Rückgang der $\mathrm{H}_{2} \mathrm{~S}$-Kapazitäten infolge zusätzlich anwesenden und koadsorbierenden Kohlendioxids (Abschnitt 4.3.2) ist der Inhomogenität des Adsorbens und der begrenzten Zahl der verfügbaren Plätze geschuldet, die bei einer Verwendung der Henry-Isothermen zur Lösung des IAST-Algorithmus nicht berücksichtigt werden. Abbildung 
46 verdeutlicht dies am Beispiel des ternären Stoffsystems $\left(\mathrm{H}_{2} \mathrm{~S}+0,5 \mathrm{~mol} \% \mathrm{CO}_{2}\right.$ in $\left.\mathrm{CH}_{4}\right)$ für die Adsorption am Silica-Alumina-Gel.

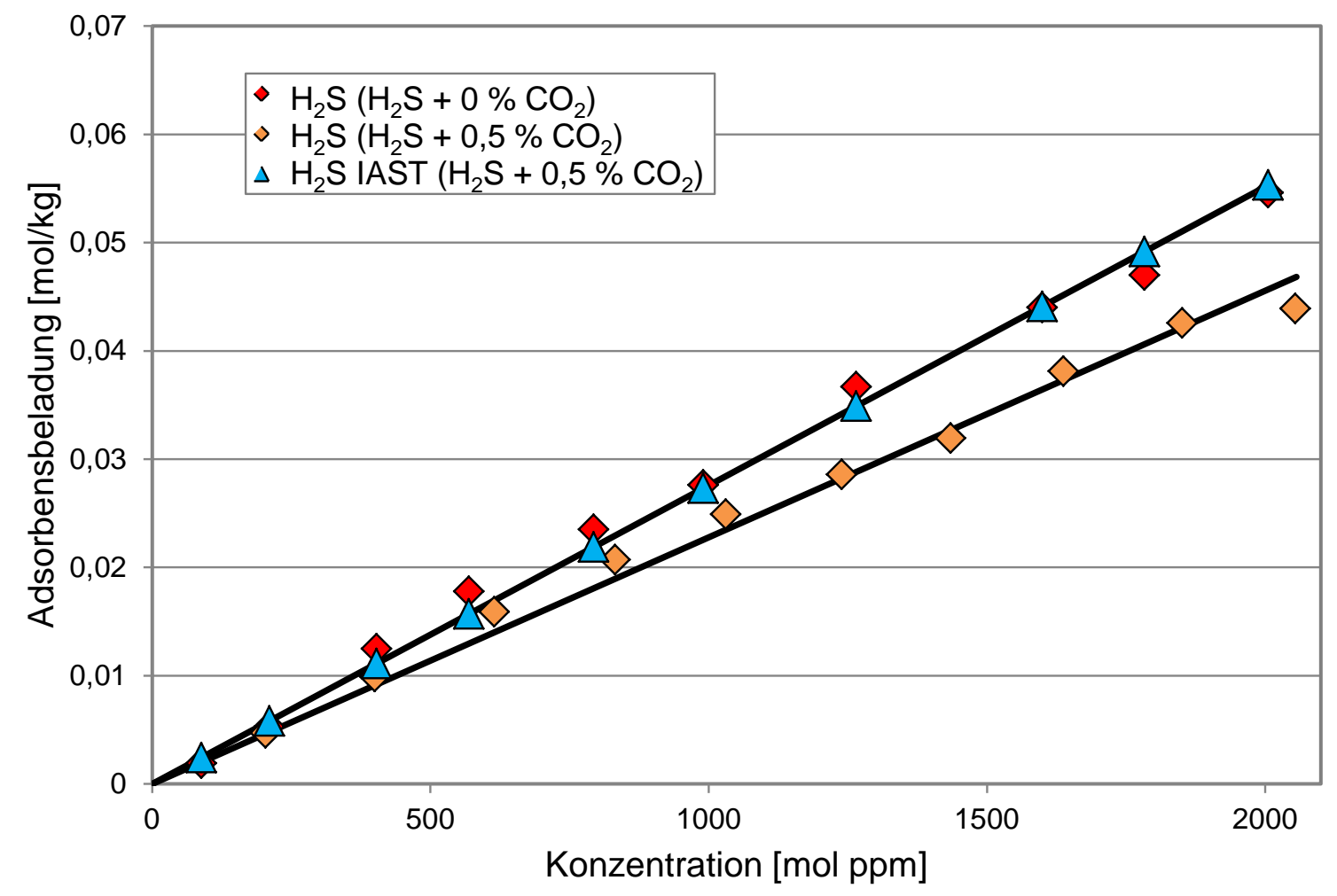

Abbildung 46: Vergleich der experimentellen Befunde mit der Vorhersage nach IAST für das Stoffsystem $\mathrm{H}_{2} \mathrm{~S}+0,5 \mathrm{~mol} \% \mathrm{CO}_{2}$ in $\mathrm{CH}_{4}$ / Silica-Alumina-Gel

Die Abbildung gibt das oben beschriebene Verhalten wieder. Die IAST-Vorhersage der $\mathrm{H}_{2} \mathrm{~S}$ Beladung (blaue Dreiecke) des ternären Stoffsystems entspricht der Beladung des binären Systems (rote Rauten). Die experimentellen Befunde weichen davon ab (orange Rauten). 


\section{$5 \quad$ Simulation von Adsorptionsprozessen}

\subsection{Rahmenbedingungen}

Das in Abschnitt 2.5 vorgestellte physikalische Modell zur Beschreibung dynamischer Adsorptionsprozesse in einem kontinuierlich durchströmten Festbett, das sich aus zwei gekoppelten partiellen Differentialgleichungen zusammensetzt, wird mit Hilfe des Programms Aspen Custom Modeler numerisch gelöst.

Die Massenbilanzen für die fluide und feste Phase enthalten drei orts- und zeitabhängige Unbekannte: die Konzentration des Adsorptivs in der Gasphase $\mathrm{c}_{\mathrm{A}}$, die Beladung des Adsorbens $X$ und die Gleichgewichtsbeladung $X_{G l}$.

Zusätzlich zu den Massenbilanzen wird demzufolge zur Lösung des Gleichungssystems eine dritte Gleichung benötigt. Die zu jeder Zeit in jedem Inkrement der Rechendomäne bei der jeweiligen Gasphasenkonzentration $\mathrm{c}_{\mathrm{A}}$ vorliegende Gleichgewichtsbeladung $\mathrm{X}_{\mathrm{Gl}}$ (vgl. Gleichung 2.35) wird mit Hilfe der experimentell bestimmten Isothermengleichung ausgedrückt.

Der in der Massenbilanz enthaltene effektive Stoffdurchgangskoeffizient gibt Aufschluss über die Kinetik des Stofftransports bei der Adsorption (vgl. Abschnitt 2.1.3). Dies ist der Parameter, der in der Simulation angepasst wird, um mit Hilfe des Modells das Experiment beschreiben zu können (vgl. Abschnitt 5.2).

Im Rahmen der Simulation von Durchbruchskurven wurden die binären Stoffsysteme, die für die beiden Standardadsorbentien experimentell untersucht wurden, betrachtet. Hierbei wurde der Schwerpunkt auf die Schwefelverbindungen und Kohlendioxid gelegt. Folgende Adsorptive wurden betrachtet: $\mathrm{CO}_{2}, \mathrm{COS}, \mathrm{H}_{2} \mathrm{~S}, \mathrm{CH}_{3} \mathrm{SH}, \mathrm{C}_{2} \mathrm{H}_{5} \mathrm{SH}$.

Zur Berechnung der axialen Dispersion nach Gleichung 2.39 werden binäre Diffusionskoeffizienten verwendet. Diese können nach Fuller [48] gemäß Gleichung 2.34 für die betrachteten Adsorptive bestimmt werden. Tabelle 24 gibt einen Überblick über die entsprechenden Diffusionskoeffizienten. 
Tabelle 24: Berechnete Diffusionskoeffizienten nach Fuller (vgl. Gleichung 2.34)

\begin{tabular}{|r|c|}
\hline Adsorptiv & $\begin{array}{c}\text { Diffusionskoeffizienten des Adsorptivs } \\
\text { im Trägergas } \mathbf{C H}_{4} \\
{\left[\mathbf{m}^{2} / \mathbf{s}\right]}\end{array}$ \\
\hline $\mathbf{C O}_{2}$ & $1,36^{\star} 10^{-5}$ \\
\hline $\mathbf{C O S}$ & $1,11^{\star} 10^{-5}$ \\
\hline $\mathbf{H}_{2} \mathbf{S}$ & $1,42^{\star} 10^{-5}$ \\
\hline $\mathbf{C H}_{3} \mathbf{S H}$ & $1,11^{\star} 10^{-5}$ \\
\hline $\mathbf{C}_{2} \mathbf{H}_{5} \mathbf{S H}$ & $9,45^{\star} 10^{-6}$ \\
\hline
\end{tabular}

\subsection{Methodik der Anpassung}

Bei der Approximation des Modells an die experimentellen Daten wurde iterativ vorgegan-

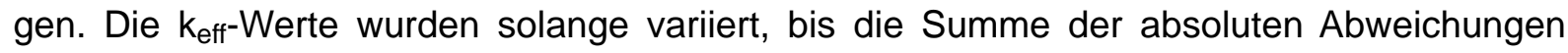
zwischen den Messpunkten der Durchbruchskurve und den entsprechenden Simulationswerten minimal wurde. Die Minimierung der Summe der relativen Abweichungen führte zu einer stärkeren Gewichtung des geringen Konzentrationsbereiches und damit zu einer schlechteren Beschreibung des Experiments. Die Vorgehensweise soll anhand Abbildung 47 beispielhaft erläutert werden.

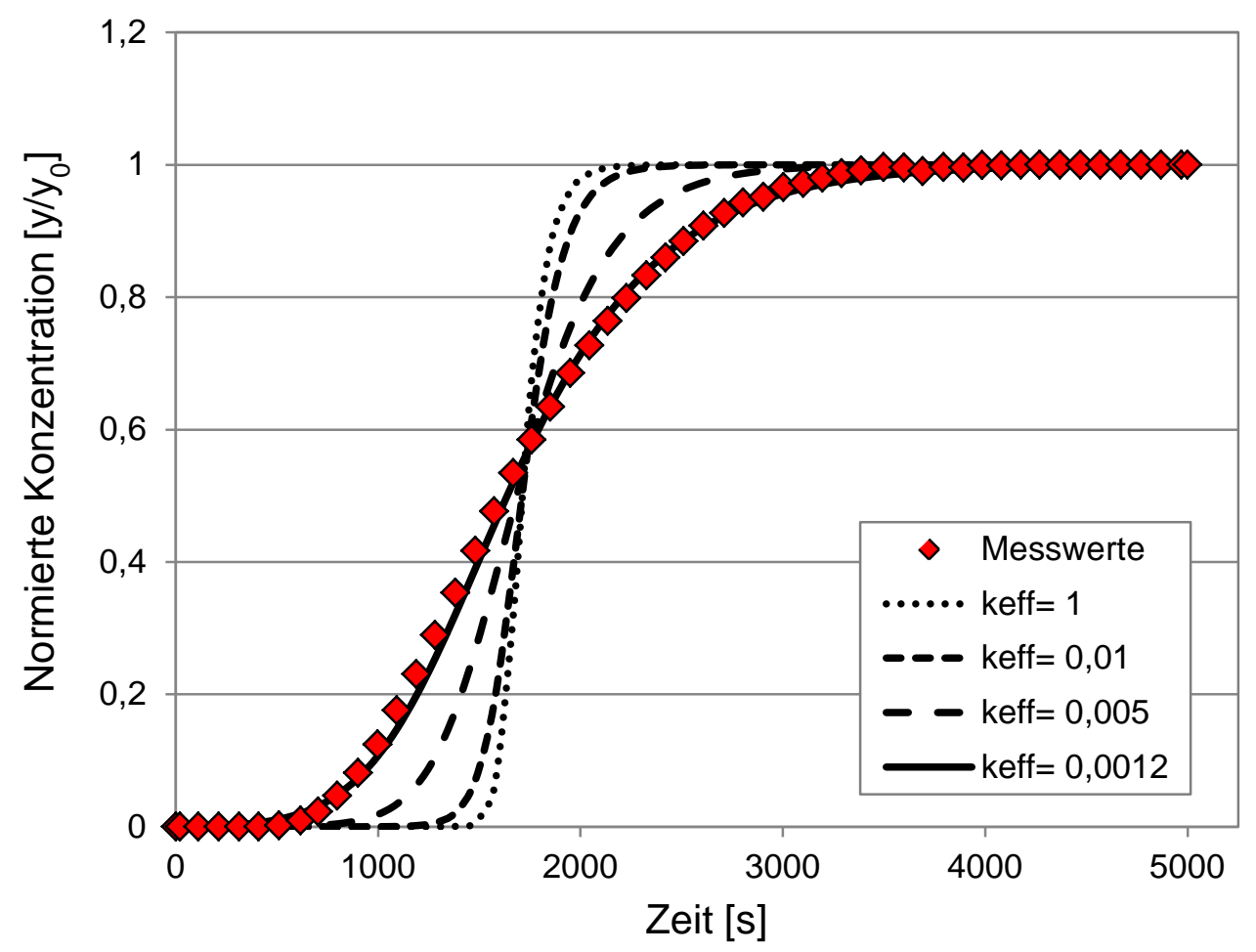

Abbildung 47: Iterative Anpassung der $\mathrm{k}_{\text {eff-Werte }}$ an eine experimentell bestimmte Durchbruchskurve am Beispiel $\mathrm{CO}_{2}$ in $\mathrm{CH}_{4} / 5 \mathrm{~A}$ Zeolith 
Die Abbildung zeigt für das Stoffsystem $\mathrm{CO}_{2}$ in $\mathrm{CH}_{4} / 5 \mathrm{~A}$ Zeolith auf die Eingangskonzentration des Beispiels normierte Durchbruchskurven. Die Messdaten werden am besten für $k_{\text {eff }}=$ 0,0012 wiedergegeben.

\subsection{Kinetikstudien für binäre Stoffsysteme}

\subsubsection{Adsorptionskinetik für Zeolith 5A}

Die Langmuir-Isotherme, mit der die Adsorption auf dem Zeolithen gut beschrieben werden (vgl. Abschnitt 4.1.3), nähert sich mit steigender Konzentration des Adsorptivs in der fluiden Phase einem Beladungsgrenzwert. Somit ist die Steigung der Langmuir-Isotherme nicht konstant, sondern wird mit steigender Konzentration des Adsorptivs geringer, bis sie bei Erreichen des Beladungsgrenzwertes gegen Null geht. Nach Gleichung 2.38 sollte die Wanderungsgeschwindigkeit für kleine Konzentrationen und Beladungen des Adsorptivs aufgrund der großen Steigung der Isotherme klein sein und zu höheren Konzentrationen und Beladungen hin ansteigen. Die neu und schwach beladenen, vorauslaufenden Bereiche der Massentransferzone wandern langsamer als die bereits stark beladenen, nachlaufenden Bereiche. Dies führt zu einer Aufsteilung der Durchbruchskurve mit zunehmender Wanderungsdauer. Bei einer hohen Gleichgewichtsbeladung ist dieser Effekt deutlicher ausgeprägt als bei einer geringen Gleichgewichtsbeladung.

Dieses Verhalten lässt sich am Beispiel der vermessenen $\mathrm{CO}_{2}$-Isotherme am Zeolithen deutlich erkennen (Abbildung 48). Die Durchbruchskurve ist bei der höchsten Gleichgewichtsbeladung am steilsten. 

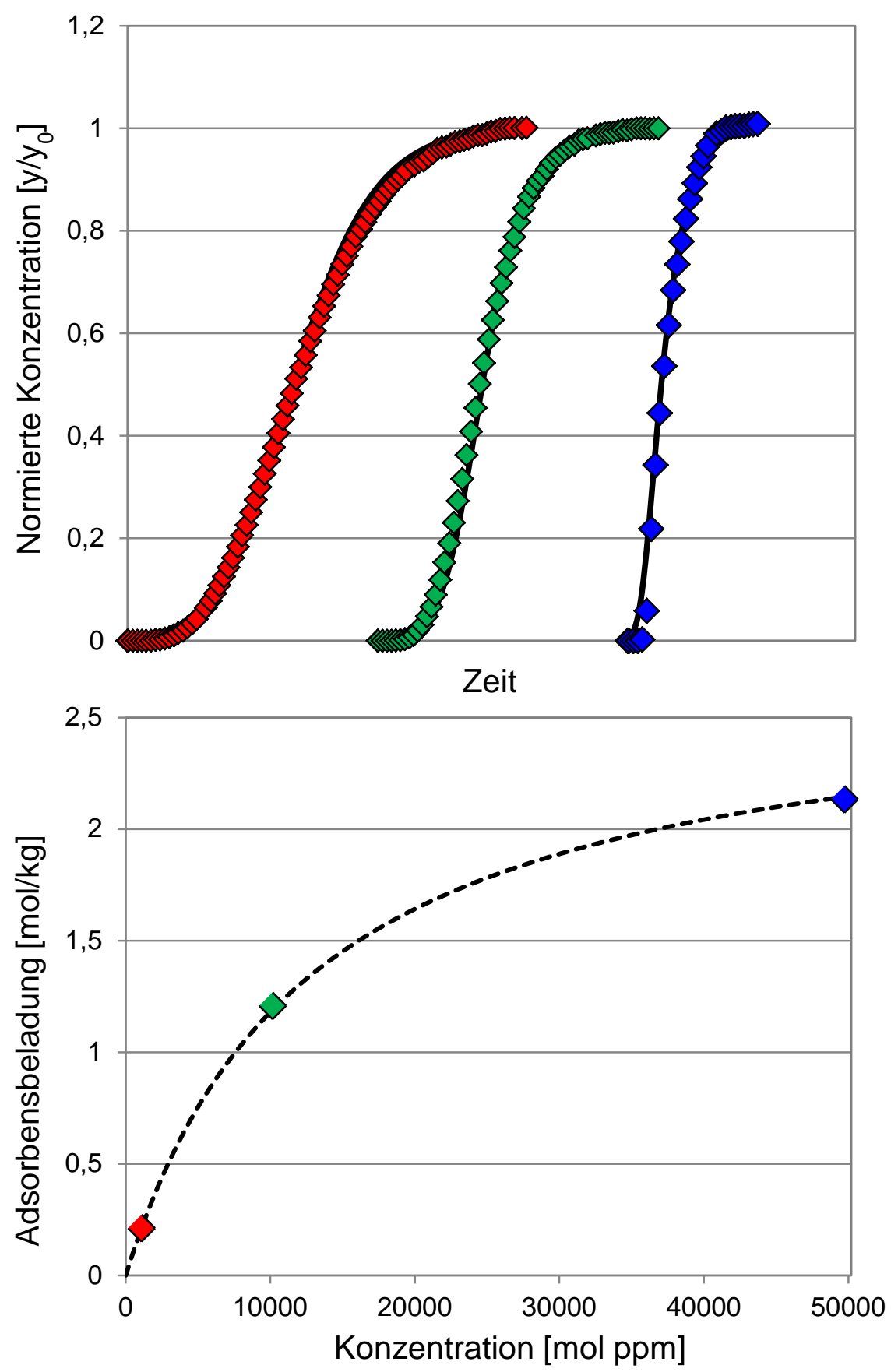

Abbildung 48: Oben: Normierte gemessene und modellierte Durchbruchskurven für die Adsorption von $\mathrm{CO}_{2}$ aus $\mathrm{CH}_{4}$ am Zeolithen des Typs 5A. Symbole: Messwerte; Schwarze Linien: Modellierung. Unten: Entsprechende $\mathrm{CO}_{2}$-Isotherme mit Messwerten. Symbole: Messwerte; Gestrichelte Linie: Langmuir-Anpassung

Der Wert für $k_{\text {eff }}$ sollte nach Gleichung 2.36 im Falle einer Langmuir-Isotherme für kleine Konzentrationen des Adsorptivs aufgrund der großen Steigung der Isotherme klein sein und zu höheren Konzentrationen hin - wenn der Summand mit der Steigung im Nenner gegen 
Null geht - aufgrund des konstant werdenden Nenners einen höheren Grenzwert erreichen. Tabelle 25 zeigt den Wertebereich der gefundenen Stoffdurchgangskoeffizienten.

Tabelle 25: Effektive Stoffdurchgangskoeffizienten für den 5A Zeolithen

\begin{tabular}{|l|c|}
\hline Adsorptiv & $\mathbf{k}_{\text {eff }}\left[\mathbf{k g} /\left(\mathbf{m}^{2 *} \mathbf{S}\right)\right]$ \\
\hline $\mathbf{C O}_{2}$ & $1,8^{\star} 10^{-3}-1,2^{\star} 10^{-3}$ \\
\hline $\mathbf{C O S}$ & $3,5^{\star} 10^{-4}-2,9^{\star} 10^{-4}$ \\
\hline $\mathbf{H}_{2} \mathbf{S}$ & $2,8^{\star} 10^{-4}-2,3^{\star} 10^{-4}$ \\
\hline $\mathbf{C H} \mathbf{S H}$ & $2,5^{\star} 10^{-4}-1,2^{\star} 10^{-4}$ \\
\hline
\end{tabular}

Auf Grund der sehr langsamen Gleichgewichtseinstellung im Falle des binären Systems $\mathrm{C}_{2} \mathrm{H}_{5} \mathrm{SH}$ in $\mathrm{CH}_{4}$ konnten die Durchbruchskurven dieses Stoffsystems nicht mit dem hier verwendeten Modell beschrieben werden.

Abbildung 49 veranschaulicht schematisch die erwartete Form der Korrelation zwischen $k_{\text {eff }}$ und der Konzentration im Vergleich zu dem gefundenen Verlauf. Es zeigt sich, dass bei hohen Konzentrationen wie erwartet ein Grenzwert erreicht wird. Bei kleinen Konzentrationen ist $k_{\text {eff }}$ jedoch größer und nicht wie erwartet kleiner als der Grenzwert.

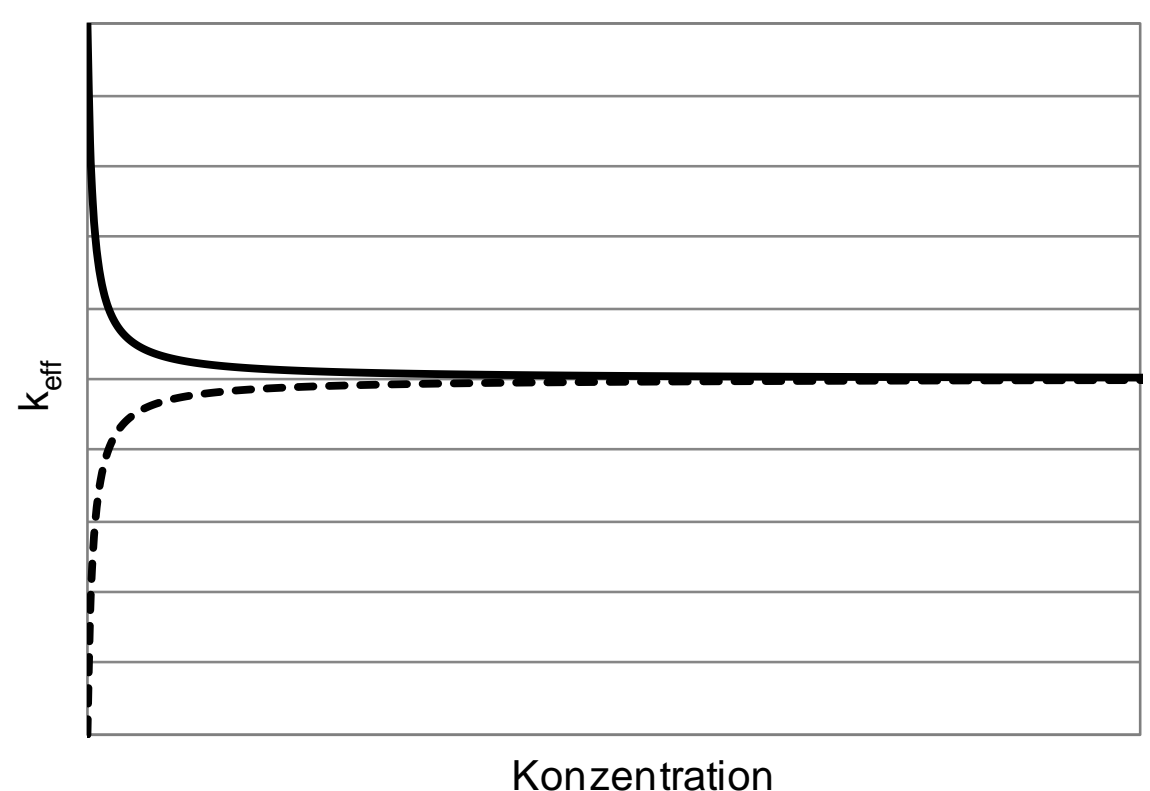

Abbildung 49: Schematischer Verlauf des effektiven Stoffdurchgangskoeffizienten in Abhängigkeit von der Konzentration. Schwarze Linie: Beobachteter Verlauf für die modellierten Stoffsysteme; Gestrichelte Linie: Erwarteter Verlauf gemäß Gleichung 2.36 
Abbildung 50 zeigt zusätzlich eine Auftragung der durch die Modellierung gefundenen $\mathrm{k}_{\mathrm{eff}^{-}}$ Werte gegen die Konzentration.

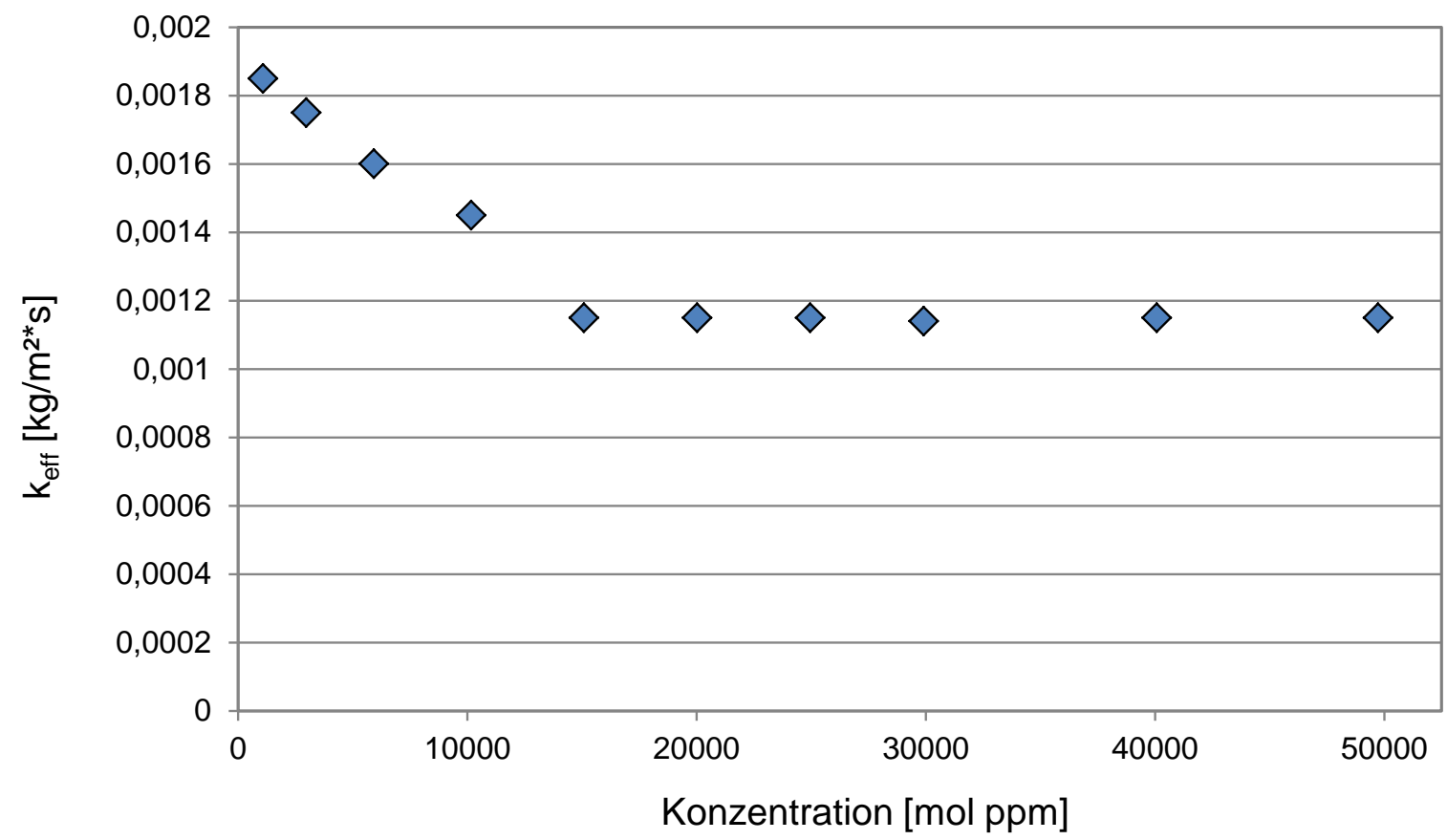

Abbildung 50: Angepasste $k_{\text {eff }}$-Werte für Durchbruchskurven bei verschiedenen Konzentrationen für das Stoffsystem $\mathrm{CO}_{2}$ in $\mathrm{CH}_{4}$ am $5 \mathrm{~A}$ Zeolith

Die Ursache für diese Abweichung vom erwarteten Verhalten bei dem mikroporösen Zeolithen könnte in einer vereinfachenden Annahme des LDF-Modells liegen. Dort wird für den diffusiven Stoffdurchgang ein pauschaler Transportwiderstand angenommen, der unabhängig von der Beladung des Adsorbens ist. In einem Mikroporensystem vergrößert sich der Transportwiderstand der Diffusion aber mit zunehmendem Porenfüllungsgrad, weil die Diffusion infolge der bei höheren Beladungen enger werdenden Porendurchmesser zunehmend gehemmt wird [38]. Die Form der Durchbruchskurve wird dann von zwei sich kompensierenden Faktoren bestimmt. Betrachtet man einen Gleichgewichtspunkt im höheren Konzentrationsbereich, so findet man einerseits aus den oben genannten Gründen eine Aufsteilung der Durchbruchskurve. Andererseits kommt es aufgrund der gehemmten Diffusion zu einer Verlangsamung der Kinetik in den höher beladenen Bereichen, deren Wanderung damit langsamer wird. Dies führt zu einer Verbreiterung der Massentransferzone und damit ähnlich wie im Falle einer ausgeprägten axialen Dispersion zu einer flacheren Durchbruchskurve. Die Durchbruchskurven bei höheren Konzentrationen in Abbildung 48 wären demnach ohne Hemmung durch verlangsamte Diffusion noch steiler. Für Durchbruchskurven bei geringeren Konzentrationen wirkt sich der weniger gehemmte Stofftransport begünstigend auf die Form der Durchbruchskurve aus. Wäre in diesem Be- 
reich die Hemmung durch verlangsamte Diffusion dominanter, dann wäre auch die Durchbruchskurve noch flacher und der resultierende Stoffdurchgangskoeffizient kleiner. Dieses Verhalten kann von einem pauschalen Parameter wie $k_{\text {eff }}$ nicht wiedergegeben werden.

\subsubsection{Adsorptionskinetik für Silica-Alumina-Gel}

\section{Systeme mit Adsorption nach der Henry-Gleichung}

Wenn das Adsorptionsgleichgewicht durch eine Henry-Isotherme mit konstanter Steigung beschrieben werden kann, ist nach Gleichung 2.38 die Wanderungsgeschwindigkeit der Konzentrationsfront unabhängig von der Höhe der erreichten Beladung im Bett. Damit ist auch die Form der Durchbruchskurve unabhängig von der Beladung. Bei unterschiedlicher Wanderungsgeschwindigkeit würde man hingegen eine Abhängigkeit der Steilheit der Durchbruchskurve von der erreichten Beladung erwarten.

In den Experimenten hat sich gezeigt, dass die gemessenen Durchbruchskurven nach einer Normierung auf die Eingangskonzentration nahezu deckungsgleich übereinander liegen und der Adsorptionsvorgang dementsprechend die gleiche Kinetik aufweist. Dieses Verhalten lässt sich am Beispiel der vermessenen $\mathrm{H}_{2} \mathrm{~S}$-Isotherme erkennen. Alle Durchbruchskurven haben die gleiche charakteristische Form (Abbildung 51). 

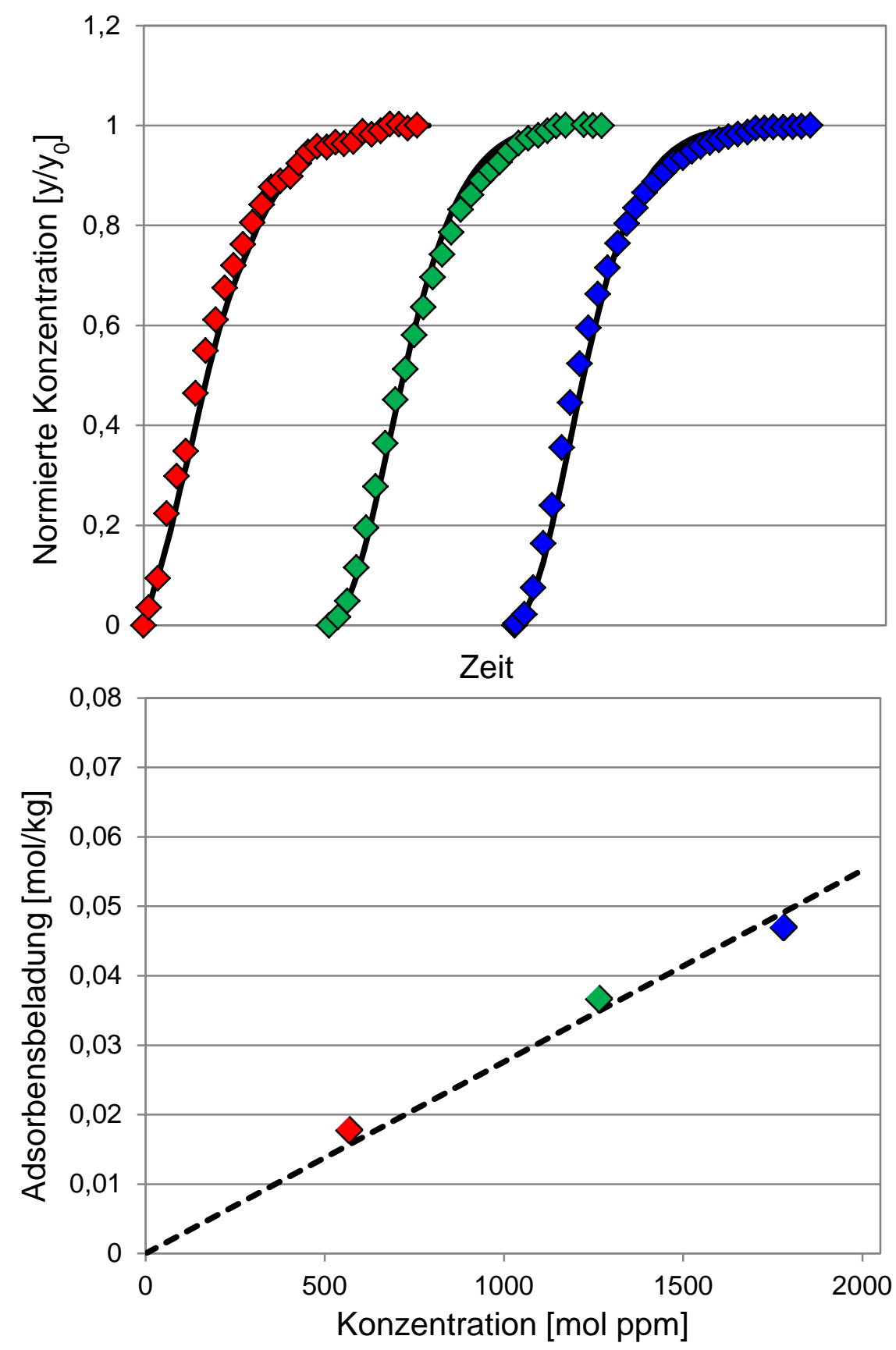

Abbildung 51: Oben: Normierte gemessene und modellierte Durchbruchskurven für die Adsorption von $\mathrm{H}_{2} \mathrm{~S}$ aus $\mathrm{CH}_{4}$ am Silica-Alumina-Gel. Symbole: Messwerte; Schwarze Linien: Modellierung. Unten: Entsprechende $\mathrm{H}_{2} \mathrm{~S}$-Isotherme. Symbole: Messwerte; Gestrichelte Linie: Henry-Anpassung

Nach Gleichung 2.36 ist im Falle einer konstanten Isothermensteigung für $\mathrm{k}_{\text {eff }}$ ebenfalls ein konstanter Wert zu erwarten. Dies hat sich bei den Experimenten bestätigt. Für die drei gemessenen Henry-Isothermen am mesoporösen Silica-Alumina-Gel $\left(\mathrm{CO}_{2}, \mathrm{H}_{2} \mathrm{~S}, \mathrm{COS}\right)$ wurden für den betrachteten Konzentrationsbereich bei der Modellierung konstante Werte für $k_{\text {eff }}$ gefunden. Tabelle 26 gibt eine Auflistung dieser Werte. 
Tabelle 26: Effektive Stoffdurchgangskoeffizienten der Henry-Systeme am Silica-Alumina-Gel

\begin{tabular}{|l|c|}
\hline Adsorptiv & keff $\left[\mathbf{k g} /\left(\mathbf{m}^{2 *} \mathbf{s}\right)\right]$ \\
\hline $\mathbf{C O}_{2}$ & $3,6^{\star} 10^{-2}$ \\
\hline $\mathbf{C O S}$ & $6,5^{\star} 10^{-3}$ \\
\hline $\mathbf{H}_{2} \mathbf{S}$ & $5,0^{\star} 10^{-3}$ \\
\hline
\end{tabular}

\section{Systeme mit Adsorption nach der Freundlich-Gleichung}

Wird das Adsorptionsgleichgewicht durch die Freundlich-Gleichung beschrieben, ergibt sich gemäß Gleichung 2.36 im Gegensatz zur linearen Henry-Gleichung eine veränderliche Kinetik. Die Freundlich-Isotherme besitzt im vorliegenden Fall (konkave Krümmung) in Ursprungsnähe eine große Steigung, die im weiteren Verlauf zu höheren Konzentrationen abnimmt. Daraus sollten sich für die effektiven Stoffdurchgangskoeffizienten der FreundlichSysteme am mesoporösen Silica-Alumina-Gel zu höheren Konzentrationen steigende $\mathrm{k}_{\mathrm{eff}^{-}}$ Werte beobachten lassen. Tabelle 27 listet die gefundenen $k_{\text {eff }}$ Werte für Methyl- und Ethylmercaptan auf.

Tabelle 27: Effektive Stoffdurchgangskoeffizienten der Freundlich-Systeme am Silica-Alumina-Gel

\begin{tabular}{|l|c|}
\hline Adsorptiv & $\mathbf{k}_{\mathrm{eff}}\left[\mathbf{k g} /\left(\mathbf{m}^{\mathbf{2}} \mathbf{s}\right)\right]$ \\
\hline $\mathbf{C H}_{3} \mathbf{S H}$ & $1,1 * 10^{-3}-2,2^{\star} 10^{-3}$ \\
\hline $\mathbf{C}_{2} \mathbf{H}_{5} \mathbf{S H}$ & $4,2^{\star} 10^{-4}-6,0^{\star} 10^{-4}$ \\
\hline
\end{tabular}

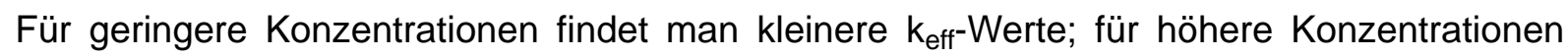
entsprechend größere $k_{\text {eff }}$ Werte. Ein solches Ergebnis ist nach der LDF-Theorie (vgl. Abschnitt 2.1.3) zu erwarten. Die Durchbruchskurven zeigen wie für den Fall der betrachteten Langmuir-Systeme ein Aufsteilen zu höheren Konzentrationen hin (vgl. Abbildung 48).

Auf Grund der mesoporösen Struktur des Silica-Alumina-Gels ist offenbar das Adsorptionsgleichgewicht der einzige einflussnehmende Parameter auf die Adsorption der Mercaptane. Der Veränderung der Stofftransporthemmung infolge der Adsorption kommt eine untergeordnete Rolle zu. Ein zunehmender Widerstand der Diffusion auf Grund des steigenden Bedeckungsgrades der Adsorbensoberfläche durch Adsorptivmoleküle wie im Falle der Adsorption auf dem Zeolith ist in den Mesoporen des Silica-Alumina-Gels nicht zu erwarten. 


\subsubsection{Vergleich der modellierten Systeme}

Wie in Gleichung 2.36 zu erkennen, setzt sich die Gesamtdiffusion aus zwei einzelnen Diffusionsmechanismen zusammen, die abhängig von der Porengröße und der Molekülgröße der Adsorptive in unterschiedlicher Ausprägung auftreten können. Für die freie Gasdiffusion und die Molekularbewegung nach Knudsen wurden in Abschnitt 2.1.3 Gleichungen 2.32 und 2.33 angegeben.

Anhand der Knudsen-Zahl lässt sich abschätzen, welcher Mechanismus dominant ist. Für die Grenze zwischen Freier Diffusion und Knudsen-Diffusion findet man in der Literatur $\mathrm{Kn}=10$. Für Knudsen-Zahlen, die deutlich unter dieser Grenze liegen, ist die Freie Diffusion der dominierende Transportmechanismus. Für Molekül/Adsorbens-Systeme, deren KnudsenZahlen deutlich über dieser Grenze liegen, tritt vorwiegend Molekularbewegung nach Knudsen auf. Tabelle 28 zeigt die Ergebnisse der Abschätzung von Knudsen-Zahlen nach einer Korrelation von Hirschfelder [85].

Tabelle 28: Nach Hirschfelder abgeschätzte Knudsen-Zahlen

\begin{tabular}{|c|c|c|c|c|c|}
\hline Adsorbens & COS & $\mathbf{C O}_{2}$ & $\mathbf{H}_{2} \mathbf{S}$ & $\mathbf{C H}_{3} \mathbf{S H}$ & $\mathbf{C}_{2} \mathbf{H}_{5} \mathbf{S H}$ \\
\hline Silica-Alumina-Gel & 9,82 & 13,42 & 12,89 & 8,15 & 6,60 \\
\hline 5A Zeolith & 51,1 & 69,77 & 67 & 42,36 & 34,34 \\
\hline
\end{tabular}

Es zeigt sich, dass sich der Stofftransport im mesoporösen Silica-Alumina-Gel für die betrachteten Adsorptive im Übergangsbereich von freier Diffusion zur Knudsen-Diffusion befindet. Für den mikroporösen Zeolithen kann für die betrachteten Moleküle hingegen von Knudsen-Diffusion als vorherrschendem Stofftransportmechanismus im Porensystem der Partikel ausgegangen werden.

Die Dominanz der langsameren Knudsen-Diffusion im Mikroporensystem des Zeolith lässt

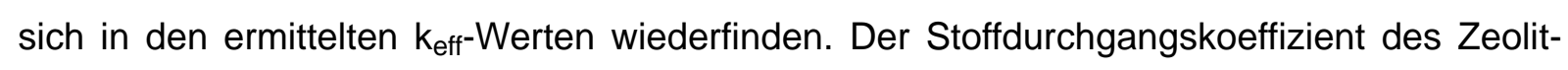
hen $\left(\mathrm{k}_{\mathrm{eff}} \approx 10^{-4}-10^{-3} \mathrm{~kg} \mathrm{~m}^{-2} \mathrm{~s}^{-1}\right)$ ist um eine Zehnerpotenz geringer als im Silica-Alumina-Gel $\left(\mathrm{k}_{\mathrm{eff}} \approx 10^{-3}-10^{-2} \mathrm{~kg} \mathrm{~m}^{-2} \mathrm{~s}^{-1}\right)$. 


\subsection{Kinetische Effekte bei ternären Stoffsystemen}

Im Folgenden Abschnitt werden kinetische Effekte bei ternären Stoffsystemen diskutiert. Da für die im Rahmen der Arbeit untersuchten ternären Stoffsysteme vergleichbare Phänomene auftreten und beschrieben werden können, beschränkt sich die Diskussion auf das ternäre Stoffsystem $\mathrm{H}_{2} \mathrm{~S}+\mathrm{CO}_{2}$ in $\mathrm{CH}_{4}$.

\section{Kinetische Effekte bei linearer Henry-Isotherme am Beispiel des Stoffsystems $\mathrm{H}_{2} \mathrm{~S}+\mathrm{CO}_{2}$ in $\mathrm{CH}_{4}$ an Silica-Alumina-Gel}

Anhand des in Abschnitt 4.4.1 diskutierten Stoffsystems lassen sich unter Berücksichtigung des LDF-Ansatzes [49] wichtige kinetische Effekte diskutieren.

Es konnte bereits anhand der Durchbruchskurven gezeigt werden, dass anwesendes $\mathrm{CO}_{2}$ einen signifikanten Einfluss auf das Durchbruchsverhalten und dementsprechend auch auf das Adsorptionsgleichgewicht des $\mathrm{H}_{2} \mathrm{~S}$ hat. Bei genauerer Betrachtung der $\mathrm{CO}_{2}$-Fronten und dementsprechend auch der $\mathrm{CO}_{2}$-Kapazitäten lässt sich feststellen, dass sich die Fronten der ternären Stoffsysteme wie die Fronten für vergleichbare Konzentrationen des binären Stoffsystems $\left(\mathrm{CO}_{2}\right.$ in $\left.\mathrm{CH}_{4}\right)$ verhalten. Dieses Verhalten lässt sich gut anhand eines Vergleichs entsprechender Kurven verdeutlichen. Abbildung 52 zeigt die Durchbruchskurven dieser Systeme.
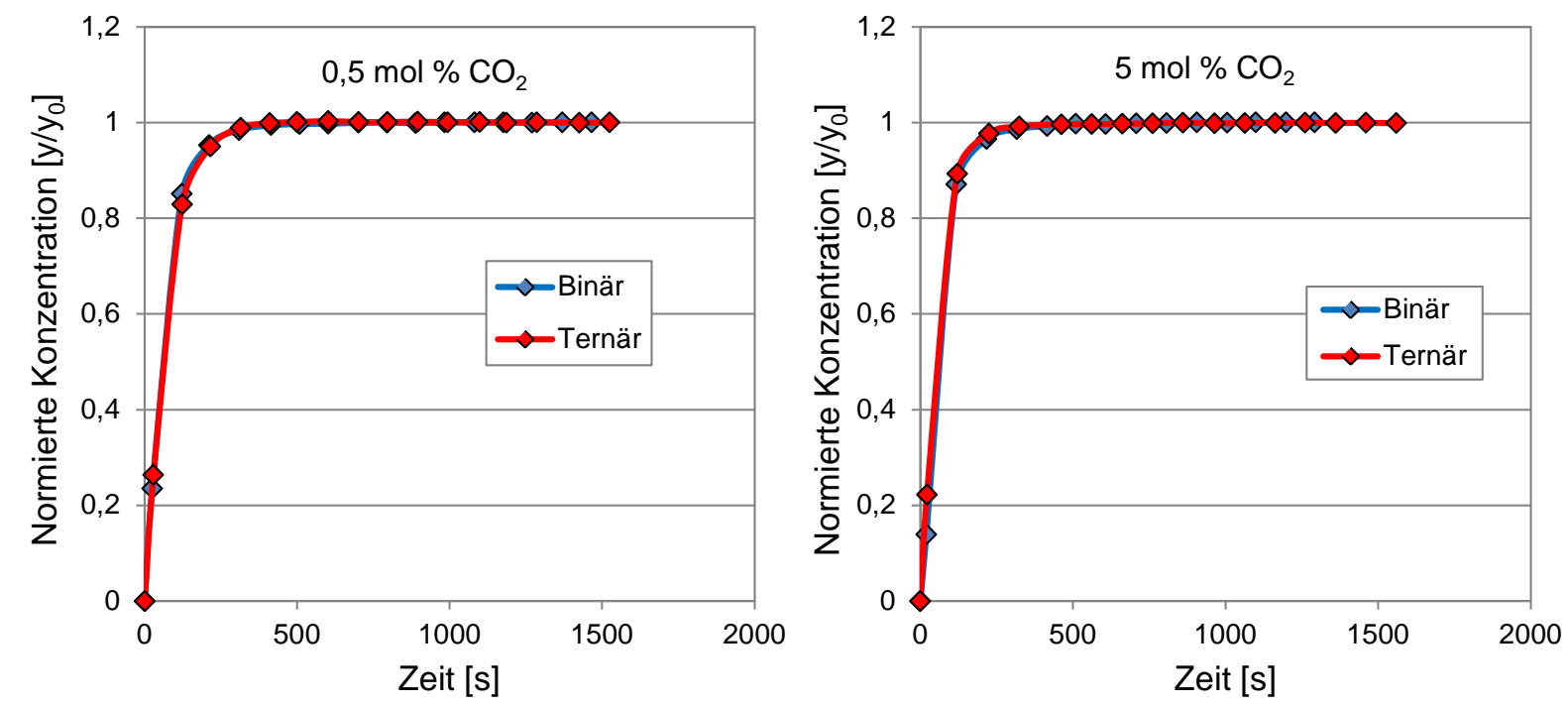

Abbildung 52: Vergleich normierter Durchbruchskurven der Adsorption von $\mathrm{CO}_{2}$ für die binären Experimente $\left(0,5 \mathrm{~mol} \% \mathrm{CO}_{2}\right.$ in $\mathrm{CH}_{4}, 5 \mathrm{~mol} \% \mathrm{CO}_{2}$ in $\left.\mathrm{CH}_{4}\right)$ und die ternären Stoffsysteme $\left(\mathrm{H}_{2} \mathrm{~S}+0,5\right.$ mol \% in $\mathrm{CH}_{4}, \mathrm{H}_{2} \mathrm{~S}+5 \mathrm{~mol} \% \mathrm{CO} 2$ in $\mathrm{CH}_{4}$ ) 
Alle dargestellten Kurven verlaufen kongruent. Das Durchbruchsverhalten des $\mathrm{CO}_{2}$ ist für beide Stoffsysteme gleich. Das ternäre Stoffsystem kann auf Grund der sehr schnellen Kinetik des $\mathrm{CO}_{2}$ als binäres Stoffsystem mit alleiniger $\mathrm{CO}_{2}$-Adsorption aufgefasst werden. Die nachlaufend adsorbierende $\mathrm{H}_{2} \mathrm{~S}$-Front beeinflusst demnach weder die Kinetik noch das Gleichgewicht des $\mathrm{CO}_{2}$. Dieses Verhalten ist eine Besonderheit bei Stoffsystemen, die durch die Henry-Gleichung beschrieben werden können.

Entsprechend der LDF-Theorie [49] ist der Verlauf der $\mathrm{CO}_{2}$-Konzentrationsfront unabhängig von der Eingangskonzentration, da die die Kinetik mitbestimmende Gleichgewichtsisotherme als Henry-Isotherme eine von der Eingangskonzentration unabhängige Steigung aufweist. Gemäß Gleichung 2.36 ist die Isothermensteigung eine der die Kinetik bestimmenden Größen.

Im Gegensatz zu den $\mathrm{CO}_{2}$-Fronten der ternären Systeme sind die $\mathrm{H}_{2} \mathrm{~S}$-Fronten nicht mehr kongruent zum binären Stoffsystem (vgl. Abbildung 35), da sie durch die vorlaufenden $\mathrm{CO}_{2}$ Fronten beeinflusst werden. Ihr Verlauf ist abhängig von der Konzentration des koadsorbierenden $\mathrm{CO}_{2}$ steiler und der Zeitpunkt der Gleichgewichtseinstellung ist früher. Durch das bereits adsorbierte $\mathrm{CO}_{2}$ stehen dem $\mathrm{H}_{2} \mathrm{~S}$ weniger Plätze zur Verfügung. Dadurch wird die Kinetik des $\mathrm{H}_{2} \mathrm{~S}$ in Anwesenheit des $\mathrm{CO}_{2}$ schneller. Dies lässt sich durch Betrachtung der zugehörigen Gleichgewichte nachvollziehen (vgl. Abbildung 29). Die $\mathrm{H}_{2} \mathrm{~S}$-Isothermen der ternären Systeme sind ebenso wie die des binären Systems Henry-Isothermen. Allerdings ergeben sich durch die reduzierten Kapazitäten auch kleinere Henry-Parameter bzw. Isothermensteigungen. Gemäß der LDF-Theorie resultiert dies in schnelleren Kinetiken. Hierbei weist die $\mathrm{H}_{2} \mathrm{~S}$-Isotherme mit $5 \mathrm{~mol} \% \mathrm{CO}_{2}$ in der Gasmischung die geringste Steigung auf. Dies führt zur steilsten Durchbruchskurve mit der größten Steigung.

\section{Kinetische Effekte bei Langmuir-Isotherme am Beispiel des Stoffsystems $\mathrm{H}_{2} \mathrm{~S}+$ $\mathrm{CO}_{2}$ in $\mathrm{CH}_{4}$ an $5 \mathrm{~A}$ Zeolith}

Im Gegensatz zu den Beobachtungen zum ternären Stoffsystem auf dem Silica-Alumina-Gel kommt es im Falle des Zeolithen zu einer gegenseitigen Beeinflussung der $\mathrm{CO}_{2}$ - und $\mathrm{H}_{2} \mathrm{~S}$ Fronten. Zur besseren Übersicht sind die Durchbruchskurven und die Isothermen nochmals in Abbildung 53 dargestellt. 

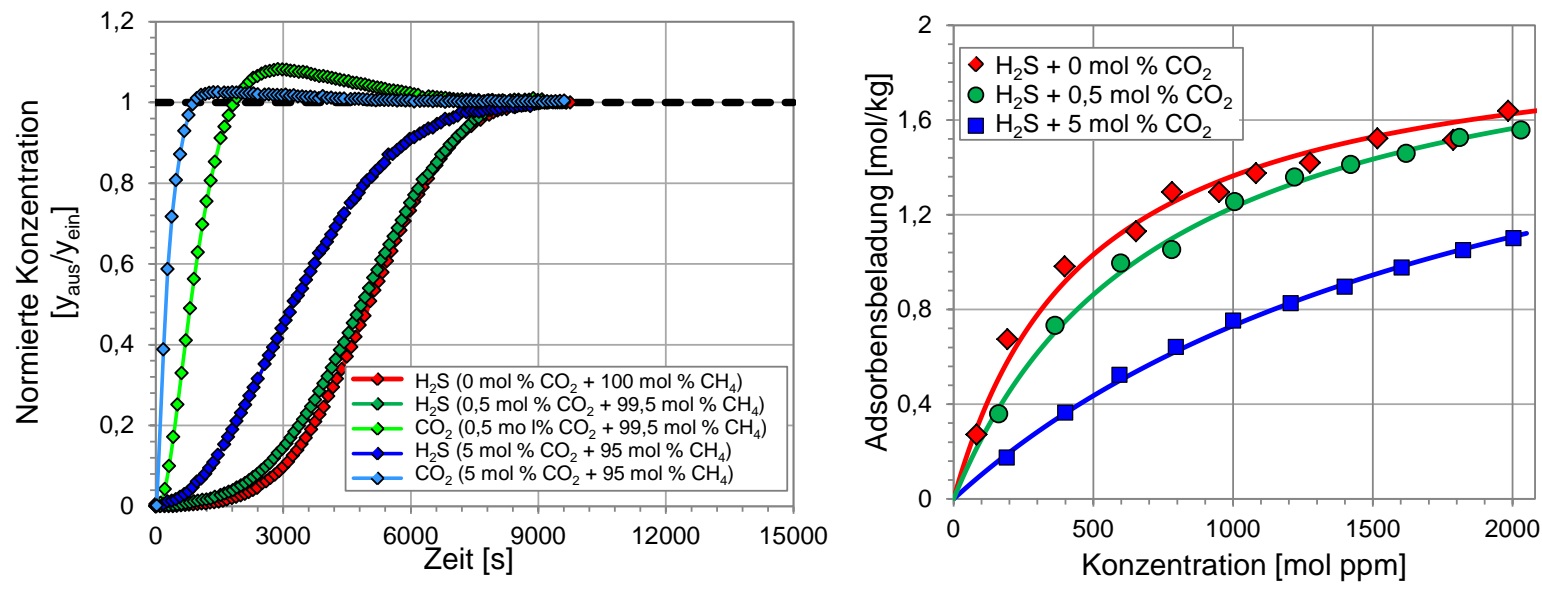

Abbildung 53: Erneute Darstellung der Abbildung 33 und Abbildung 39

Für beide ternären Untersuchungen wird $\mathrm{CO}_{2}$ mit der schneller durch die Schüttung laufenden Front von der nachlaufenden, langsameren $\mathrm{H}_{2} \mathrm{~S}$-Font teilweise verdrängt. Dies zeigt sich im Überschwingen der $\mathrm{CO}_{2}$-Konzentration im austretenden Gasstrom Ein gewisser Anteil des $\mathrm{CO}_{2}$ gelangt dabei wieder in die freie Gasphase. Einige der adsorbierten $\mathrm{CO}_{2}$-Moleküle verbleiben jedoch im Porensystem des Adsorbens. Die $\mathrm{CO}_{2}$-Fronten für die beiden unterschiedlichen Konzentrationen sind im Gegensatz zum beobachteten Verhalten auf dem Silica-Alumina-Gel nun nicht mehr kongruent. Da das $\mathrm{CO}_{2}$-Adsorptionsverhalten des binären Systems $\left(\mathrm{CO}_{2}\right.$ in $\left.\mathrm{CH}_{4}\right)$ auf dem Zeolithen anhand einer Langmuir-Isotherme beschrieben werden kann, ist es nachvollziehbar, dass die $\mathrm{CO}_{2}$-Fronten im ternären Fall ebenfalls ein Langmuir-Verhalten zeigen. Gemäß der LDF-Theorie kommt es in einem solchen Fall zu einem Aufsteilen der Durchbruchskurven bei höheren Konzentrationen (vgl. Abschnitt 2.1.3), da nun die veränderliche Isothermensteigung die Adsorptionskinetik beeinflusst.

Auf Grund des koadsorbierenden $\mathrm{CO}_{2}$ stehen dem $\mathrm{H}_{2} \mathrm{~S}$ im Vergleich zum binären Stoffsystem weniger Adsorptionsplätze zur Verfügung. Die Form der $\mathrm{H}_{2} \mathrm{~S}$-Durchbruchskurve ist daher verändert.

Je nach $\mathrm{CO}_{2}$-Gehalt der Gasmischung ist die Veränderung der $\mathrm{H}_{2} \mathrm{~S}$-Front unterschiedlich stark ausgeprägt. Für die geringere $\mathrm{CO}_{2}$-Konzentration in der Gasmischung $(0,5 \mathrm{~mol} \%)$ ist die Anfangssteigung der $\mathrm{H}_{2} \mathrm{~S}$-Front etwas steiler. Im Bereich der Gleichgewichtseinstellung ähnelt die Kurve stark der entsprechenden Kurve des binären Systems.

Für die höhere $\mathrm{CO}_{2}$ Konzentration in der Gasmischung ist die Beeinflussung stärker ausgeprägt. Die $\mathrm{H}_{2} \mathrm{~S}$-Durchbruchskurve im System mit $5 \mathrm{~mol} \% \mathrm{CO}_{2}$ weist die größte Anfangssteigung auf. Vergleicht man die $\mathrm{H}_{2} \mathrm{~S}$-Isothermen, lässt sich erkennen, dass die $\mathrm{H}_{2} \mathrm{~S}$ Isotherme im System mit $5 \mathrm{~mol} \% \mathrm{CO}_{2}$ in der Gasmischung die flachste Steigung in Ur- 
sprungsnähe besitzt, was in der schnellsten Kinetik resultieren sollte. Im Bereich hoher Konzentrationen hingegen ist die Isothermensteigung größer als diejenigen des binären und des anderen ternären Systems, die beide bereits deutlicher in ein Beladungsplateau verlaufen zu scheinen. Als Konsequenz daraus ergibt sich im Bereich der Gleichgewichtseinstellung im Vergleich zu den beiden anderen Systemen ein flacherer Verlauf der Durchbruchskurve. 


\section{$6 \quad$ Zusammenfassung und Ausblick}

Ziel der vorliegenden Arbeit war es, die Vorgänge bei der adsorptiven Gasentschwefelung im kontinuierlich durchströmten Festbett besser zu verstehen. Die gewonnenen Erkenntnisse lassen sich in zwei Teilbereiche zusammenfassen:

i. Experimentelle Untersuchungen der adsorptiven Aufreinigung eines methanreichen Gases (z.B. Erdgas) Silica-Gelen und einem 5A Zeolith

ii. Modellierung von Durchbruchskurven und Vorhersage von Mehrkomponentengleichgewichten.

\subsection{Zusammenfassung}

Zur experimentellen Untersuchung der adsorptiven Entschwefelung wurde im Rahmen der Arbeit eine kontinuierlich durchströmte Festbettversuchsanlage aufgebaut und in Betrieb genommen.

Zunächst wurde die Adsorption einer Verbindung aus dem Trägergas untersucht. Aus einem Vergleich dieser binären Stoffsysteme lässt sich für beide verwendeten Adsorbentien eine eindeutige Tendenz herleiten: Je polarer das Adsorptivmolekül ist, desto besser adsorbiert es an den polaren Adsorbentien. Für die untersuchten Adsorptive ergibt sich die Reihenfolge der gefundenen Adsorptionskapazitäten:

$$
\mathrm{H}_{2} \mathrm{O}>\mathrm{C}_{3} \mathrm{H}_{7} \mathrm{SH}>\mathrm{C}_{2} \mathrm{H}_{5} \mathrm{SH}>\mathrm{CH}_{3} \mathrm{SH}>\mathrm{H}_{2} \mathrm{~S}>\mathrm{COS}>\mathrm{CO}_{2}
$$

Für das große Molekül Ethylmercaptan konnten bei der Untersuchung des mikroporösen $5 \mathrm{~A}$ Zeolithen sterische Effekte beobachtet werden.

Wie zu erwarten war, wurden am Zeolith für alle Adsorptive im untersuchten Konzentrationsbereich deutlich höhere Adsorptionskapazitäten erreicht als für das Silica-Alumina-Gel.

Zum Einfluss des Trägergases auf den Adsorptionsprozess wurden ergänzende Untersuchungen durchgeführt. Am Beispiel des Adsorptivs Wasser wurde die Adsorption sowohl aus dem Trägergas Methan als auch aus Stickstoff für beide Adsorbentien vermessen. Im Falle des mesoporösen Silica-Alumina-Gels zeigt sich keine Abhängigkeit der erzielten $\mathrm{H}_{2} \mathrm{O}$ Kapazitäten vom verwendeten Trägergas. Für beide Trägergase ergeben sich vergleichbare Beladungen. Für den mikroporösen Zeolithen des Typs $5 \mathrm{~A}$ werden bei der Verwendung von 
Stickstoff als Trägergas im Vergleich zu Methan deutlich höhere $\mathrm{H}_{2} \mathrm{O}-\mathrm{Kapazitäten} \mathrm{gefunden.}$ Als maßgebliche Gründe hierfür wurde eine Koadsorption des Methans und von Verunreinigungen im Trägergas vorgeschlagen, die im Zeolith ausgeprägter ist als im Silica-AluminaGel.

Zur Annäherung an reale Erdgaszusammensetzungen und zur Untersuchung des Einflusses zusätzlich in der Gasmischung enthaltener Komponenten auf die Abtrennleistung der betrachteten Adsorbentien wurde das binäre Stoffsystem $\mathrm{H}_{2} \mathrm{~S}$ in $\mathrm{CH}_{4}$ um weitere Adsorptive ergänzt und auf diese Weise ternäre Stoffsysteme $\left(\mathrm{H}_{2} \mathrm{~S}+\mathrm{CO}_{2}\right.$ in $\mathrm{CH}_{4}, \mathrm{H}_{2} \mathrm{~S}+\mathrm{H}_{2} \mathrm{O}$ in $\left.\mathrm{CH}_{4}\right)$ erzeugt. Für beide ternäre Stoffsysteme kommt es abhängig von Konzentration und Verbindung zu unterschiedlich stark ausgeprägten $\mathrm{H}_{2} \mathrm{~S}$-Kapazitätsverlusten. Die bei der Adsorption stattfindenden Koadsorptions- und Verdrängungseffekte konnten experimentell nachgewiesen und anhand kinetischer Daten diskutiert werden. Abhängig von der das Gleichgewicht beschreibenden Isothermen-Gleichung der beteiligten Verbindungen konnten unterschiedliche kinetische Effekte beobachtet werden. Es zeigte sich auch für die Untersuchungen der ternären Stoffsysteme eine ausgeprägte Korrelation zwischen der Polarität der konkurrierenden Adsorptive und der verbleibenden Beladungskapazität des $\mathrm{H}_{2} \mathrm{~S}$.

Untersuchungen mit binären und ternären Stoffsystemen an speziell hergestellten SilicaAlumina-Gel-Varianten mit unterschiedlichem Aluminiumgehalt konnten zeigen, dass ein Zusammenhang zwischen dem Aluminiumgehalt des Adsorbens und der Entschwefelungsleistung am Beispiel von $\mathrm{H}_{2} \mathrm{~S}$ besteht. Die Adsorption von $\mathrm{CO}_{2}$ aus Methan an den verschiedenen Silica-Alumina-Gel-Varianten konnte mit strukturellen Eigenschaften aus BETAnalysen korreliert werden. Je geringer der Aluminium-Gehalt des Adsorbens ist, desto größer sind BET-Oberfläche und $\mathrm{CO}_{2}$-Kapazität. Für $\mathrm{H}_{2} \mathrm{~S}$ ergeben sich im Vergleich zum Standardmaterial deutlich geringere Kapazitäten. In Untersuchungen mit ternären Stoffsystemen zeigen sich geringere $\mathrm{H}_{2} \mathrm{~S}$-Kapazitätsverluste für die modifizierten Materialien als für das Standardmaterial. Diese Befunde können mit spektroskopisch nachgewiesenen, unterschiedlich koordinierten Aluminiumplätzen, die unterschiedliche Selektivitäten zeigen, erklärt werden.

Die kinetischen Versuchsdaten binärer Stoffsysteme wurden mit Hilfe eines auf dem LDFAnsatz basierenden physikalischen Modells beschrieben. Trotz der simplen Modellstruktur wurden eine gute Übereinstimmung zwischen Modell und Experiment gefunden und effektive Stoffdurchgangskoeffizienten bestimmt. Die verschiedenen Einflussfaktoren auf die Adsorpti- 
onskinetik wurden diskutiert. Abhängig von der Isothermen-Gleichung, die das Gleichgewicht beschreibt, und dem vom Porensystem des Adsorbens abhängigen dominanten Stofftransportmechanismus konnten unterschiedliche kinetische Verhalten festgestellt werden.

Am Beispiel der Stoffsysteme $\mathrm{H}_{2} \mathrm{~S}+\mathrm{CO}_{2}$ in $\mathrm{CH}_{4}$ wurden für beide Adsorbentien bekannte Mehrkomponentenvorhersagemodelle angewendet. Für den Vergleich mit den experimentellen Befunden der Langmuir-Systeme auf dem 5A Zeolithen ergeben sich für die Modelle nach Myers und Prausnitz und Markham und Benton im Falle der geringen $\mathrm{CO}_{2^{-}}$ Konzentration in der Gasmischung gute Übereinstimmungen in den Vorhersagen der $\mathrm{H}_{2} \mathrm{~S}$ Kapazitäten. Für das in den jeweiligen Gasmischungen mit konstanter Konzentration vorliegende $\mathrm{CO}_{2}$ ergeben sich deutlich größere Abweichungen der Modelle zu den experimentell ermittelten Werten. Ebenfalls für das ternäre Stoffsystem mit höherer $\mathrm{CO}_{2}$-Konzentration ergaben sich deutliche Abweichungen.

Die im Falle der ternären Stoffsysteme für das Silica-Alumina-Gel nachgewiesene konkurrierende Adsorption von $\mathrm{H}_{2} \mathrm{~S}$ und $\mathrm{CO}_{2}$ und der damit verbundene Kapazitätsrückgang des $\mathrm{H}_{2} \mathrm{~S}$ im Vergleich zum binären Fall kann durch die IAS-Theorie nicht erfasst werden. Werden beide binären Isothermen der beteiligten Verbindungen durch eine Henry-Gleichung beschrieben, ergeben sich aus der IAST-Berechnung für den ternären Fall die binären Kapazitäten. Der Grund dafür ist, dass bei der Adsorption nach der Henry-Theorie unendlich viele verfügbare Plätze und keine wechselseitige Beeinflussung der adsorbierenden Moleküle angenommen werden.

\subsection{Kritik an der eigenen Arbeit}

Die im Rahmen der Arbeit aufgestellte Reihenfolge der Adsorptionskapazitäten, nach welcher eine Verbindung besser adsorbiert, je polarer sie ist, kann auf die industrielle Praxis nur behutsam angewendet werden. Streng genommen gilt diese Reihenfolge nur für die untersuchten Stoffsysteme. Für Moleküle von ähnlicher molekularer Dimension und ähnlichen Eigenschaften lassen sich jedoch zumindest Beladungstendenzen vorhersagen oder Kapazitäten abschätzen. Die Untersuchungen zu ternären Stoffsystemen geben Hinweise darauf, welche Konkurrenz zwischen verschiedenen Adsorptiven besteht. Diese Befunde können qualitativ als Anhaltspunkt für andere Stoffsysteme dienen. Beispielsweise ist für ein ternäres Stoffsystem mit Wasser und Mercaptanen mit vergleichbaren Effekten wie für die im Rahmen der Arbeit untersuchten ternären Stoffsysteme zu rechnen. 
Weiterhin muss bedacht sein, dass sowohl die Gleichgewichte als auch die kinetischen Daten unter sehr genau definierten Betriebsbedingungen und unter Verwendung hochreiner Stoffe ermittelt wurden. Eine reale Erdgasaufbereitungsanlage kann zum einen starken Schwankungen in ihren Betriebsbedingungen unterliegen. Zum anderen kann das aufgegebene Rohgas sehr viele Verbindungen enthalten und in seiner Zusammensetzung stark variieren. Dies macht eine exakte Auslegung eines Prozesses schwierig. Dennoch sind die im Rahmen der Arbeit generierten Daten von großer Wichtigkeit. Die bestimmten Gleichgewichte für die Adsorption einer Verbindung aus dem Trägergas Methan sind im Vergleich zur Reingasadsorption einer Verbindung als realitätsnäher einzustufen und können daher für eine realistischere Auslegung eines Adsorptionsprozesses dienen.

Für die Adsorption von Wasser konnte anhand eines Trägergaswechsels gezeigt werden, dass das Trägergas, aus welchem das Wasser adsorbiert wird, im Falle des Zeolithen einen deutlichen Einfluss auf die $\mathrm{H}_{2} \mathrm{O}$-Kapazität hat. Für das untersuchte Silica-Alumina-Gel tritt dieser Effekt nicht auf. Auf Grund fehlender Daten zur Adsorption reinen Stickstoffs und reinen Methans lässt sich die Diskussion zum Einfluss des Trägergases nicht durch experimentelle Daten untermauern. Zwar ist unter Berücksichtigung von Literaturwerten [20] zu vermuten, dass Methan gegenüber Stickstoff bei Umgebungstemperatur (293 K) im Falle des Zeolithen eine deutlich größere Affinität zur polaren Adsorbensoberfläche aufweist. Dies konnte anhand der Adsorption von Wasser aus den beiden Trägergasen für dieses Adsorbens nachgewiesen werden. Eine quantitative Bewertung für die vorliegenden Untersuchungen lässt dies jedoch nicht zu.

Weiterhin wurde die Trägergasvariation lediglich für Experimente mit dem sehr polaren Adsorptiv Wasser untersucht. Adsorbierendes Wasser wird in der Lage sein, einen Großteil des auf Grund der experimentellen Prozedur (vgl. Abschnitt 3.2.3) auf der Oberfläche befindlichen Methans zu verdrängen. Zieht man die Adsorption einer weniger affinen Verbindung in Betracht, könnte der Einfluss durch eine mit Methan vorbeladene Oberfläche auf die Gleichgewichtsbeladung des Adsorptivs stärker ausgeprägt sein. Hierzu sind weitere Untersuchungen notwendig.

Die im Falle der untersuchten Silica-Alumina-Gele geführte Diskussion zur Rolle des Aluminiums für die $\mathrm{H}_{2} \mathrm{~S}$-Adsorption aus einem binären und einem ternären Gasgemisch bedarf weiterer Untersuchungen. Die aufgestellte Hypothese, nach der es bei der Herstellung der Adsorbentien zur Bildung unterschiedlich selektiver Aluminium-Adsorptionsplätzen kommt, ist 
zwar wahrscheinlich, konnte jedoch im Rahmen dieser Arbeit nicht durch einen experimentellen Nachweis verifiziert werden. Hierzu müssten zusätzliche Festkörper-NMRUntersuchungen durchgeführt und ein Vergleich mit dem Standardmaterial angestellt werden.

Reale Erdgasaufbereitungsverfahren zur Entschwefelung werden üblicherweise bei sehr hohen Betriebsdrücken von bis zu 100 bar betrieben $[3,86]$. Die im Rahmen dieser Arbeit durchgeführten Untersuchungen wurden bei einem Betriebsdruck von 1,3 bar durchgeführt. Dementsprechend stellt sich die Frage, inwieweit die Adsorptionsgleichgewichte auf reale Betriebsbedingungen einer Erdgasaufbereitung übertragen werden können. Betrachtet man die Adsorption einer Verbindung aus einer binären Gasmischung bei konstanter Konzentration bei zwei verschiedenen Systemdrücken, sollten sich bei idealer Gasphase und geringer Druckabhängigkeit der Wechselwirkungen in der Adsorbatphase für beide Zustände sehr ähnliche Beladungskapazitäten ergeben. Andererseits wird die Gasphase bei sehr hohen Drücken ein ausgeprägtes nicht-ideales Verhalten zeigen. Zudem können Verbindungen in der Nähe und oberhalb des kritischen Druckes auch in der adsorbierten Phase veränderte Eigenschaften aufweisen. Der kritische Druck von $\mathrm{CO}_{2}$ liegt bei 73 bar, der kritische Druck von Methan bei 46 bar und der von $\mathrm{H}_{2} \mathrm{~S}$ bei 89 bar $[87,88]$. Dies könnte zu einer druckabhängigen Beeinflussung der Adsorbensbeladung führen. Nach Erfahrungsberichten von Experten der BASF [84, 89] besteht jedoch eine gute Vergleichbarkeit zwischen den hier ermittelten Daten und Gleichgewichtswerten aus Aufbereitungsanlagen, die bei hohen Drücken betrieben werden.

\subsection{Ausblick}

Die im Rahmen dieser Arbeit untersuchten Adsorbentien werden im industriellen Maßstab in zyklisch operierenden Mehrbett-Adsorptionsanlagen zur Aufbereitung von Erdgas eingesetzt. Während in einem oder mehreren Betten der Adsorptionstakt läuft, wird in anderen Betten regeneriert und gekühlt. Bei der Reinigung von Erdgas wird dies üblicherweise thermisch in Thermal-Swing-Adsorption-Processes (TSA) durchgeführt. Je nach Adsorbens und Rohgasqualität werden die Desorptionsschritte bei Temperaturen von $300^{\circ} \mathrm{C}$ und höher betrieben. Für den wirtschaftlichen Betrieb einer solchen Anlage reicht daher die alleinige Kenntnis über den Adsorptionsprozess nicht aus. Der kostenintensivere Desorptionstakt muss ebenso betrachtet werden. Zu diesem Zweck wurde im Rahmen der Arbeiten am Lehrstuhl für Ther- 
mische Verfahrenstechnik (Prof. Bathen) eine weitere Festbett-Versuchsanlage zur Untersuchung des Desorptionsverhaltens von Adsorbentien aufgebaut und in Betrieb genommen. Mit Hilfe dieser Anlage besteht einerseits experimentell die Möglichkeit, die Adsorption binärer oder komplexerer Mischungen im Temperaturbereich zwischen $25-300^{\circ} \mathrm{C}$ zu vermessen. Aus Gleichgewichtsdaten bei unterschiedlichen Temperaturen lassen sich beispielsweise mit Hilfe der Methode der Isosteren Adsorptionsenthalpien abschätzen [90]. Weiterhin lässt sich andererseits ebenfalls die thermische Desorption (TSA/CSA) des Bettes bis $300^{\circ} \mathrm{C}$ untersuchen. Eine interessante Fragestellung ist beispielsweise, welchen Einfluss die starke Aufkonzentrierung der Adsorptive bei der Desorption hat. Eventuell bei höheren Temperaturen und höheren Konzentrationen ablaufende Reaktionen wie z.B. die in Abschnitt 4.2.1 diskutierte cos-Bildung, die in industriellen Anlagen immer noch ein Problem darstellt [89], könnten mit dem Desorptionstakt und den dort herrschenden Bedingungen zusammenhängen.

Bei Vorliegen experimenteller Gleichgewichtsdaten bei verschiedenen Temperaturen ist man außerdem in der Lage, das bestehende mathematische Modell zur Beschreibung der Adsorption um die Energiebilanzen für die fluide und feste Phase zu ergänzen. Dadurch könnten beispielsweise Adsorptionsenthalpien bestimmt und die Temperaturabhängigkeit des Stofftransports beschrieben werden. Weiterhin könnte der Desorptionstakt in das Programm integriert werden und damit Desorptionsverläufe modelliert werden.

Die Untersuchung von Gasmischungen mit mehreren adsorbierbaren Verbindungen ist weiterhin von großem Interesse. Beispielsweise im Erdgas enthaltene höhere Kohlenwasserstoffe könnten den Entschwefelungsprozess entscheidend beeinflussen. Hierzu sollten weitere Stoffsysteme vermessen werden.

Zudem ist es vorstellbar, ternäre Stoffsysteme noch detaillierter zu betrachten. Dies würde die Möglichkeit bieten, die Anwendung von Mehrkomponentenvorhersagemodellen wie $z$. B. IAST weiter zu vertiefen. Darüber hinaus könnte man nichtideale Modelle wie z. B. RAST (Real Adsorbed Solution Theory) oder VST (Vacancy Solution Theory) verwenden, um Aktivitätskoeffizienten zu bestimmen.

Für eine Optimierung des Silica-Alumina-Gels sind weitere Modifikationen denkbar. Beispielsweise könnte eine starke Erhöhung des Aluminium-Gehaltes bei der Schwefelwasserstoff-Adsorption Vorteile bei hohen Konzentrationen konkurrierender Adsorptive bieten. Die selektive Adsorption des Schwefelwasserstoffs an den Aluminiumplätzen könnte trotz der Konkurrenz durch andere Verbindungen höhere $\mathrm{H}_{2} \mathrm{~S}$-Kapazitäten gewährleisten. 


\section{Literaturverzeichnis}

[1] British Petroleum, „Statistical Review of World Energy,“ Juni 2012.

[2] „www.naturalgas.org,“ [Online]. [Zugriff am 06.07.2012].

[3] Kidnay, A. J.; Parrish, W. R. Fundamentals of Natural Gas Processing; CRC Press Taylor \& Francis Group: Boca Raton, 2006.

[4] Hofmann, F.; Plättner, A. Evaluierung der Möglichkeiten zur Einspeisung von Biogas in das Erdgasnetz, Endbericht Forschungsvorhaben im Auftrag der Fachagentur Nachwachsende Rohstoffe e. V., Leipzig, 2005.

[5] Jensen, A. B.; Webb, C. Treatment of H2S-containing gases: A review of microbiological alternatives, Enzyme Microb. Technol. 1995, 17, 2-10.

[6] Kohl, A.; Nielsen, R. Gas Purification, Fifth Edition; Gulf Publishing Company: Houston, 1997.

[7] Neumann, D. W.; Lynn, S. Oxidative Absorption of $\mathrm{H} 2 \mathrm{~S}$ and $\mathrm{O} 2$ by Iron Chelate Solutions, AIChE Journal, 1984, 1, 62-69.

[8] Sattler, K. Thermische Verfahrenstechnik, Grundlagen, Auslegung, Apparate; Dritte Auflage; Wiley-VCH: Weinheim, 2001.

[9] Sircar, S. Basic Research Needs for Design of Adsorptive Gas Separation Processes, Ind. Eng. Chem. Res. 2006, 45, 5435-5448.

[10] Abatzoglou, N.; Boivin, S. A review of biogas purification processes, Biofuels, Bioprod. Bioref 2009, 3, 42-71.

[11] Cline, C.; Hoksberg, A.; Abry, R. Biological Process for H2S Removal from Gas Streams- The Shell-Paques/Thiopaq Gas Desulfurization Process, Laurance Reid Gas Conditionig Conference, Norman (Oklahoma), 2003.

[12] Adib, F.; Bagreev, A.; Bandosz, T. J. Analysis of the Relationchip between H2S Removal Capacity and Surface Properties of Unimpregnated Activated Carbons, Environ. Sci. Technol. 2000, 34, 686-692.

[13] Adib, F.; Bagreev, A.; Bandosz, T. J. Effect of pH and Surface Chemistry on the Mechanism of H2S Removal by Activated Carbons, J.Colloid Interface Sci. 1999, 216, 360-369.

[14] Bagreev, A.; Bandosz, T. J. Role of Sodium Hydroxide in the Process of Hydrogen Sulfide Adsorption/Oxidation on Caustic-Impregnated Activated Carbons, Ind. Eng. Chem. Res. 2002, 41, 672-679. 
[15] Choi, D.-Y.; Lee, J.-W.; Jang, S.-C.; Ahn, B.-S.; Choi, D.-K. Adsorption dynamics of hydrogen sulfide in impregnated activated carbon bed, Adsorption 2008, 14, 533-538

[16] Tsai, J.-H.; Tsai, J.-H.; Hsu, Y.-C. Adsorption of Hydrogen Sulfide and Methylmercaptan Mixture Gas on Alkaline Activated Carbon, J. Chin. Inst. Chem. Eng. 1999, 3, 145152.

[17] Chiang, H.-L.; Tsai, J.-H.; Tsai, C.-L.; Hsu, Y.-C. Adsorption Characteristics of Alkaline Activated Carbon Exemplified by Water Vapor, H2S and CH3SH Gas, Sep. Sci. Technol. 2000, 35, 903-918.

[18] Fails, J. C.; Harris, W. D. Practical way to sweeten natural gas, Oil Gas J. 1960, 58, 86-90.

[19] Chi, C. W.; Lee, H. Natural Gas Purification by 5A Molecular Sieve and Its De-sign Method, AIChE Symposium Series, 69, 95-101.

[20] Groninger, G.; Hedden, K.; Rao, R. Kinetics of Removal of Impurities from Gases by High Pressure Adsorption, Chem. Eng. Technol. 1987, 10, 63-72.

[21] Maddox, R. Scavenger and Solid Bed Sweetening in Gas Conditioning and Processing Vol. 4; Campbell Petroleum Series: Norman Oklahoma, 1998.

[22] Pechler, N.; Hillmer, G.; Emig, G. Experimentelle Bestimmung und modellmäßige Beschreibung von Durchbruchskurven bei der H2S/CS2-Gemischadsorption, Chem. Ing. Tech. 1990, 62, 734-735.

[23] Sakano, T.; Tamon, H. Adsorption Selectivity of Coffee Aroma Components on Zeolite 5A, Food Sci. Technol. Int. 1996, 2, 174-179.

[24] Weber, G.; Benoit, F. Selective adsorption of ethyl mercaptan on NaX Zeolite. Microporous and Mesoporous Mater, 2008, 109, 184-192.

[25] Weber, G.; Bellat, J. P. Adsorption Equilibrium of Light Mercaptans on Faujasites, Adsorption 2005, 11, 183-188.

[26] Sarbak, Z. Desulfurization of ethanethiol over cadmium and mercury modified Zeolite NaX, Applied Catalysis A: General 1996, 147, 47-54.

[27] Ryzhikov, A.; Hulea, V.; Tichit, D. Methyl Mercaptan and carbonyl sulfide traces removal through adsorption and catalysis on zeolites and layered double hydroxides, Applied Catalysis A: General 2011, 297, 218-224.

[28] Yoon, Y. H.; Nelson, J. H. Application of gas adsorption kinetics. I. A theoretical model for respirator cartridge service life, Am. Ind. Hyg. Assoc. J. 1984, 45, 509-516.

[29] Tanada, S.; Boki, K. Adsorption Behavior Hydrogen Sulfide Inside Micropores of Molecular Sieve Carbon 5A and Molecular Sieve Zeolite 5A, Bull. Environ. Contam. Toxicol. 1982, 29, 624-629. 
[30] Zhou, L.; Zhong, L. Sorption and Desorption of a Minor Amount of H2S on Silica-Gel Covered with a thin film of Triethanolamine, Ind. Eng. Chem. Res. 2004, 43, 17651767.

[31] Keller, J. U.; Staudt, R. Gas Adsorption Equilibria, Experimental Methods and Adsorptive Isotherms; Springer: New York, 2005.

[32] Ruthven, D. M. Principles of Adsorption and Adsorption Processes; John Wiley and Sons: New York, 1984.

[33] Freundlich, H. Über die Adsorption in Lösungen, Zeitung der Physikalischen Chemie 1906, 57, 385-470.

[34] Langmuir, I. The Adsorption of Gases on Plane Surfaces of Glass, Mica and Platinum, J. Am. Chem. Soc. 1918, 40, 1361-1403.

[35] Yang, R. T. Adsorbents: Fundamentals and Applications; John Wiley \& Sons: New York, 2003.

[36] Toth, J. Adsorption Theory, Modeling, and Analysis; Marcel Dekker Inc.: New York, 2001.

[37] Do, D. D. Adsorption Analysis: Equilibria and Kinetics; Imperial College Press: London, 1998.

[38] Kast, W. Adsorption aus der Gasphase - Ingenieurwissenschaftliche Grundlagen und technische Verfahren; VCH: Weinheim, 1988.

[39] Markham, E. C.; Benton, A. F. The Adsorption of Gas Mixtures by Silica, J. Am. Chem. Soc. 1931, 53, 497-507.

[40] Myers, A. L.; Prausnitz, J. M. Thermodynamics of mixed-gas adsorption, AIChE J. $1965,11,121-127$.

[41] Lewis, W. K.; Gilliland, E. R.; Chertow, B.; Cadogan, W. P. Adsorption Equilibria Hydrocarbon Gas Mixtures, J. Ind. Eng. Chem. 1950, 42, 1317-1326.

[42] Costa, E.; Sotelo, J. L.; Calleja, G.; Marrón, C. Adsorption of binary and ternary hydrocarbon gas mixtures on activated carbon: Experimental determination and theoretical prediction of the ternary equilibrium data, AIChE J. 1981, 27, 5-12.

[43] Wilson, G. M. Vapor-Liquid Equilibrium. XI. A New Expression for the Excess Free Energy of Mixing, J. Am. Chem. Soc. 1964, 86, 127-130.

[44] Suwanayuen, S.; Danner, R. P. A gas adsorption isotherm based on vacancy solution theory, AIChE J. 1980, 26, 68-76.

[45] Hill, T. L. Theory of Multimolecular Adsorption from a Mixture of Gases, J. Chem. Phys. 1946, 14, 268-275. 
[46] Gonzalez, A. J.; Holland, C. D. Adsorption of multicomponent mixtures by solid adsorbents, AIChE J. 1970, 16, 718-724.

[47] Bathen, D.; Breitbach, M. Adsorptionstechnik; Springer-Verlag: Berlin, 2001.

[48] Fuller, E. N.; Schettler, P. D.; Giddings, J. C. A New Method for Prediction of Binary Gas-Phase Diffusion Coefficients, Ind. Eng. Chem. 1966, 58, 18-27.

[49] Sircar, S.; Hufton, J. R. Why Does the Linear Driving Force Model for Adsorption Kinetics Work?, Adsorption 2000, 6, 137-147.

[50] Mersmann, A. Adsorption, in: Ullmann's Encyclopedia of Industrial Chemistry Vol. B3; VCH Verlagsgesellschaft: Weinheim 1988.

[51] Glueckauf, E. Theory of Chromatography, Trans. Faraday Soc. 1955, 51. 15401551.

[52] Wakao, N.; Funazkri, T. Effect of Fluid Dispersion coefficients on particle-to-fluid mass transfer coefficients in packed beds, Chem. Eng. Sci. 1978, 33, 1375-1384.

[53] Bansal, R. C.; Goyal, M. Activated Carbon Adsorption; Tayler and Francis: Boca Raton, 2005.

[54] Weis, R. Kieselgel, in: Ullmann's Encyklopädie der technischen Chemie Band 21, 4. Auflage; Verlag Chemie: Weinheim 1979.

[55] Speight, J. G. Synthetic Fuels Handbook: Properties, Process, and Performance; McGraw Hill: New York, 2008.

[56] Reiffenstein, R. J.; Hulbert, W. C.; Roth, S. H. Toxicology of Hydrogen Sulfide, Annu. Rev. Pharmacol. Toxicol. 1992, 32, 109-134.

[57] Moeller, E. Handbuch Konstruktionswerkstoffe - Auswahl, Eigenschaften, Anwendung; Carl Hanser Verlag: München, 2008.

[58] Richtlinie 2001/81/EG des Europäischen Parlaments und des Rates vom 23. Oktober 2001 über nationale Emissionshöchstmengen für bestimmte Luftschadstoffe; Amtsblatt Nr. L 309, 27.11.2001, 22-30.

[59] Mortimer; C.E.; Müller, U. Chemie, 9. Auflage; Georg Thieme Verlag: Stuttgart, 2007.

[60] Hoppe, B.; Martens, J. Aminosäuren - Herstellung und Gewinnung, Chemie in unserer Zeit 1984, 18, 73-86.

[61] Lutz, W.; Seidel, A.; Boddenberg, B., On the Formation of COS from H2S and CO2 in the Presence of Zeolite/Salt Compounds, Adsorpt. Sci. and Technol. 1998, 16, 578581.

[62] Debye, P. Polare Molekeln; S. Hirzel Verlag: Leipzig, 1929.

[63] Lucas, K. Applied Statistical Thermodynamics; Springer-Verlag: Berlin, 1992. 
[64] Ramachandran, K.; Deepa, G.; Namboori, K. Computational Chemistry and Molecular Modeling, Principles and Applications; Springer-Verlag: Berlin, Heidelberg, 2008.

[65] Persönliches Gespräch mit Prof. Eckhard Spohr und Dr. Holger Somnitz, Lehrstuhl für Theoretische Chemie, Fakultät Chemie, Universität Duisburg-Essen.

[66] Butcher, J. C. Numerical Methods for Ordinary Differential Equations, Second Edition; Wiley: Weinheim, 2008.

[67] DIN ISO 9277, Determination of the specific surface area of solids by gas adsorptionBET Method.

[68] Gregg, S. J.; Sing, K. S. Adsorption, Surface Area and Porosity; Academic Press: London, 1982.

[69] Landolt-Brönstein, Numerical Data and Functional Relationships in Science and Technology, New Series, II/19c; Springer: Heidelberg, 1992.

[70] Nelson, R.; Lide, D.; Maryott, A. Selected Values of Electric Dipole Moments for Molecules in the Gas Phase, Natl. Stand. Ref. Data Ser. (U.S., Nat. Bur. Stand.) 10, 1967.

[71] Landolt-Brönstein, Numerical Data and Functional Relationships in Science and Technology, New Series, II/14a; Springer: Heidelberg, 1982.

[72] Grubner, O. Molekularsiebe; Verlag d. Wissenschaften: Berlin, 1968.

[73] Schön, W. Handbuch der reinsten Gase; Springer: Heidelberg, 2005.

[74] Lutz, W.; Buhl, J. C.; Thamm, H. A New COS-Surpressing Zeolite for Gas Sweetening, Erdöl, Erdgas Kohle, 1999, 115, 87-89.

[75] Schulthess, C. P.; Dey, D. K. Estimation of Langmuir Constants using Linear and Nonlinear Least Squares Regression Analyses, Soil Sci. Soc. Am. J. 1996, 60, 433442.

[76] Datenblatt EL-FLOW, Digitale thermische Massendurchflussmesser und- regler für Gase, Bronkhorst High Tech.

[77] Operating Instructions ALC Models Electronic Analytical Balances and Precision Scales, ACCLAB Satorius Group.

[78] Operating Instructions A-Series Electronic Analytical Balances, Fisher Scientific.

[79] Iler, R. K. The Chemistry of Silica; John Wiley \& Sons: New York, 1979.

[80] Hair, M. L. Infrared Spectroscopy in Surface Chemistry; Marcel Dekker, Inc.: New York, 1967.

[81] Jensen, W. B. The Lewis Acid-Base Concepts; John Wiley \& Sons: New York, 1980. 
[82] Wang, Y.; LeVan, M. D. Adsorption Equilibrium of Carbon Dioxide and Water Vapor on Zeolites 5A and 13X Silica-Gel: Pure Components, J. Chem. Eng. Data 2009, 54, 2839-2844.

[83] Lutz, W.; Suckow, M.; Bülow, M. Adsorption of hydrogen sulfide on molecular sieves, Gas Sep. Purif. 1990, 4, 190-196.

[84] Persönliches Gespräch mit Frau Dr. Angela Siegel, BASF Catalysts Germany GmbH.

[85] Poling, B. E.; Prausnitz, J. M.; O'Connell, J. P. The Properties of Gases and Liquids, Fifth Edition; McGraw-Hill: Ney York, 2001.

[86] Mitariten, M.; Lind, W. The Sorbead ${ }^{\mathrm{TM}}$ Quick-Cycle Process for Simultaneous Removal of Water, Heavy Hydrocarbons and Mercaptans from Natural Gas; Laurence Reid Gas Conditioning Conference: Norman Oklahoma, 2007.

[87] Cubitt, A. G.; Henderson, C.; Staveley, L. A. K.; Fonseca, I. M. A.; Ferreira, A. G. M. Some thermodynamic properties of liquid hydrogen sulphide and deuterium sulphide, J. Chem. Thermodyn.1987, 19, 703.

[88] Lide, D. R. CRC Handbook of Chemistry And Physics. 85. Auflage; CRC Press LLC: 2004.

[89] Persönliches Gespräch mit Herrn Klaus Neumann, BASF Catalysts GmbH Germany.

[90] Sievers, A. Über das Gleichgewicht der Adsorption in Anlagen zur Wasserstoffgewinnung, Dissertation 1993, TU München. 


\section{Lebenslauf}

Der Lebenslauf ist in der Online-Version aus Gründen des Datenschutzes nicht enthalten. 